\title{
Plantar Measurements to Determine Success of Surgical Correction of Stage Ilb Adult Acquired Flatfoot Deformity
}

\author{
Erika Matheis \\ Virginia Commonwealth University
}

Follow this and additional works at: https://scholarscompass.vcu.edu/etd

Part of the Biomedical Engineering and Bioengineering Commons

(C) The Author

\section{Downloaded from}

https://scholarscompass.vcu.edu/etd/2943

This Thesis is brought to you for free and open access by the Graduate School at VCU Scholars Compass. It has been accepted for inclusion in Theses and Dissertations by an authorized administrator of VCU Scholars Compass.

For more information, please contact libcompass@vcu.edu. 
Plantar Measurements to Determine Success of Surgical Correction of Stage IIb Adult Acquired Flatfoot Deformity

A thesis submitted in partial fulfillment of the requirements for the degree of Master of Science in Biomedical Engineering at Virginia Commonwealth University.

By:Erika Ashley Matheis, B.A. UNC-Chapel Hill 2008

Director: Dr. Jennifer Wayne, Director of Orthopedics Laboratory, Department of Biomedical Engineering

Virginia Commonwealth University

Richmond, Virginia

December 2012 


\section{Acknowledgement}

I would like to acknowledge several groups for their support in my education and research on this project. The first is Dr. Jennifer Wayne whom had the patience to teach engineering to an exercise and sports science major. Without her help and guidance I would not have succeeded. As my graduate advisor she has encouraged me to find my strengths in biomedical engineering, even with my slightly different approach.

Also the various members of the laboratory, specifically Meade Spratley who has helped me with everything from classroom to laboratory, always with a sarcastic attitude that only some can appreciate (like myself). I would also like to acknowledge my other lab mates (current and former) Ruchi Chande, Afsar Mir, Casey Woodcock, Sean Higgins, Kevin Elmore, and Ben Majors as we have ventured through the graduate world together and they helped to keep me sane despite many hours of studying and data analysis. And lastly, Johnny Owen, who keeps the lab together and comes up with new and interesting ideas with a smile. The entire faculty and staff of the BME department who have helped to further my learning and broaden my perspective on science. The $\mathrm{MCV}$ orthopedics department, as they have given me a chance to observe and learn the clinical application of the work we do in the laboratory. Dr. Adelaar for the partial recruitment of participants as well as the surgical correction of participants in this study. Barbara Ennis and Charlotte Barrett who help schedule all of the research performed as a bridge between the clinic and laboratory.

Finally I would like to thank my friends and family who have supported me throughout this venture in my life. Thank you, University of Richmond coaches and athletes who have supported and understood my hectic schedule. I appreciate all of my friends from near and far whom I have ever talked to about my project a bit too technically and have lent an ear and nodded their heads, specifically, Kristina, Zac, Lex, Shane, Chloe, Lindsay, and Damien. Thanks to my mentor, Dr. Klibanoff who inspired my interest in orthopedics and continues to do so. I could not have done this without the

support of my mom, Nancy, who was always just a phone call away. Thank you Mom for helping me put things in perspective and guiding me. 


\section{Table of Contents}

\begin{tabular}{ll} 
ABSTRACT & 4 \\
\hline
\end{tabular}

INTRODUCTION $\quad \mathbf{5}$



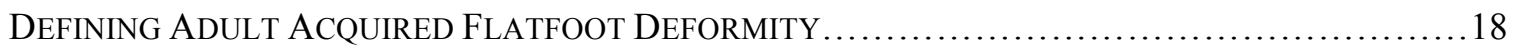

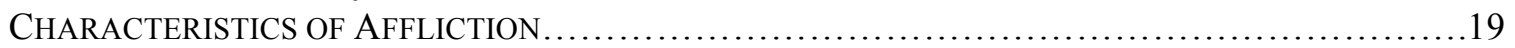

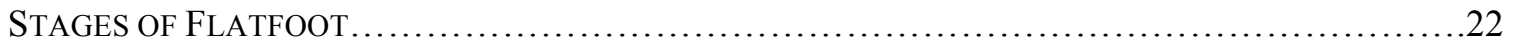



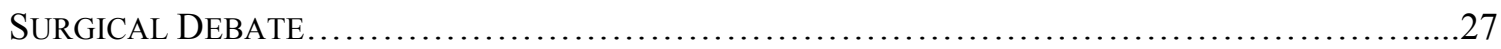

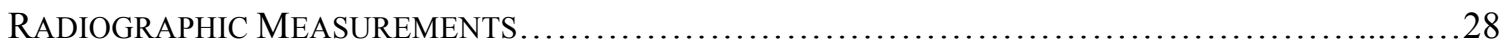

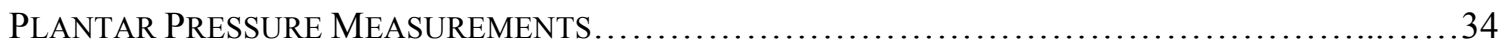

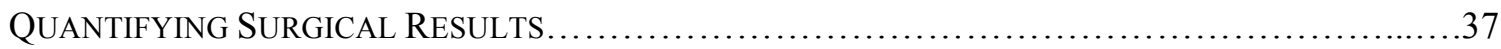

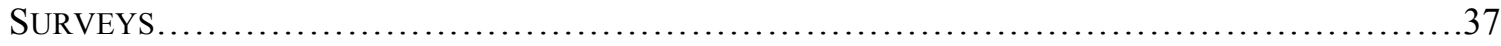

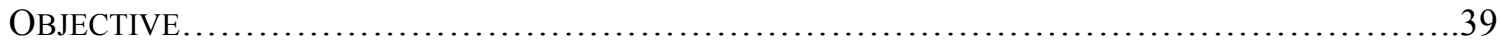

\begin{tabular}{ll} 
METHODS & 40 \\
\hline
\end{tabular}



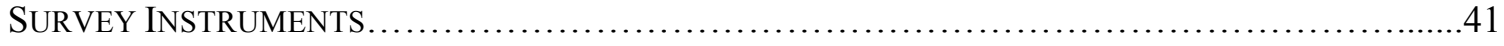

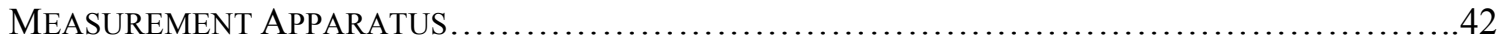

PROCEDURE. . . .

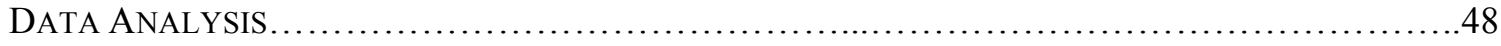

RESULTS $\quad \mathbf{5 4}$

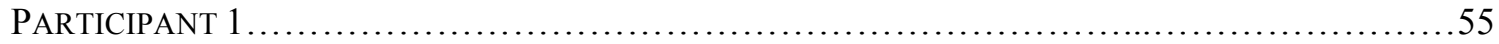

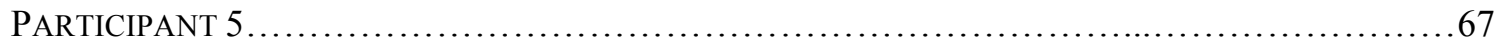

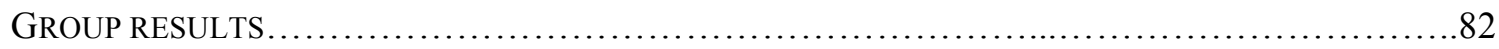

DISCUSSION \& CONCLUSIONS 96

APPENDIX A 108

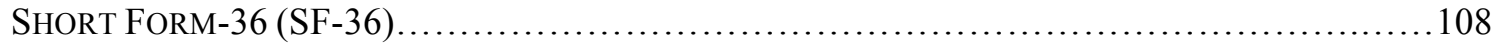

FOOT AND ANKLE OUTCOME SCORE SURVEY (FAOS) ................................ 113

APPENDIX B



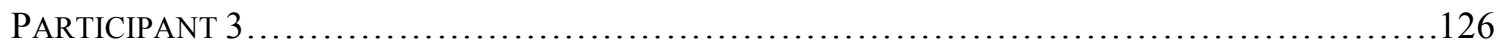

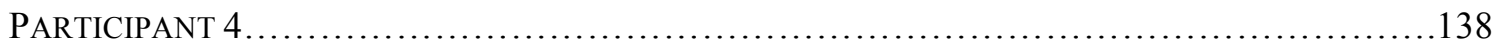

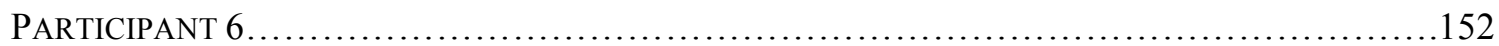

WORKS CITED $\quad 168$ 


\begin{abstract}
Adult Acquired Flatfoot Deformity (AAFD) is a progressive disease characterized by mechanical degeneration of the soft tissue structure in the arch of the foot that leads to changes in joint alignment. Surgical intervention commonly via tendon transfer and bony osteotomy is used to restore arch architecture, however there is a lack of quantitative assessments that measure the success of the surgical correction in vivo.

Using plantar pressures via Tekscan ${ }^{\circledR}$ HR Mat and surveys (SF-36, FAOS), pre-operative and post-operative measures for six participants were defined, analyzed and compared. A paired t-test showed significant lateral shift for percent body weight during walking postoperativelyin the forefoot and midfoot regions. However, arch index measurement showed no significant change. The FAOS survey score also improved statistically postoperatively. The surgical correction was successful as deemed by some of these quantitative and qualitative measures.
\end{abstract}




\section{INTRODUCTION}

The ability to walk and move defines daily life for almost everyone. Mobility through your legs dictates occupations and hobbies, from a doctor walking through the emergency room to attend to a patient, to an adventurer climbing to the top of a mountain. For such a complex process of walking, the average person does not think about the intricate muscle movements and coordinated articulation of multiple bones required to go from point to point. Walking for most people has become almost an involuntary thought (like breathing) unless something gets in the way.

During early development in childhood we are taught to stand, then walk, with a few bobbles and falls along the way. Countless hours of practicing this motion from childhood to adult life has lead to the grace and fluidity most people have while walking. The study of gait patterns has arisen so that differences can be determined and quantified. Some studies are specifically targeted with populations of elderly, children, and injured because the differences can have a large effect on participants' mobility. Since mobility defines daily life, those that have difficulty are of particular concern because lack of mobility may diminish quality of life. 


\section{ANATOMY}

The anatomy of the foot and ankle is intricate with 28 bones (including phalanges and sesmoids) including the complex joint structure of the ankle where the talus meets the tibia and fibula (McMinn, Hutchings, \& Logan, 1996) (Figure 1). Serving as the structural backbone of the foot and ankle, the bones and junctures are crucial to understanding how the foot and ankle work. The sizes of the bones are extremely diverse from the tip of the fifth phalange to the long tibia that extends through the leg. The shape of the bones are also very different to serve their biomechanical function of supporting forces on the foot and permitting motion, as well as attachment sites for soft tissue structures.

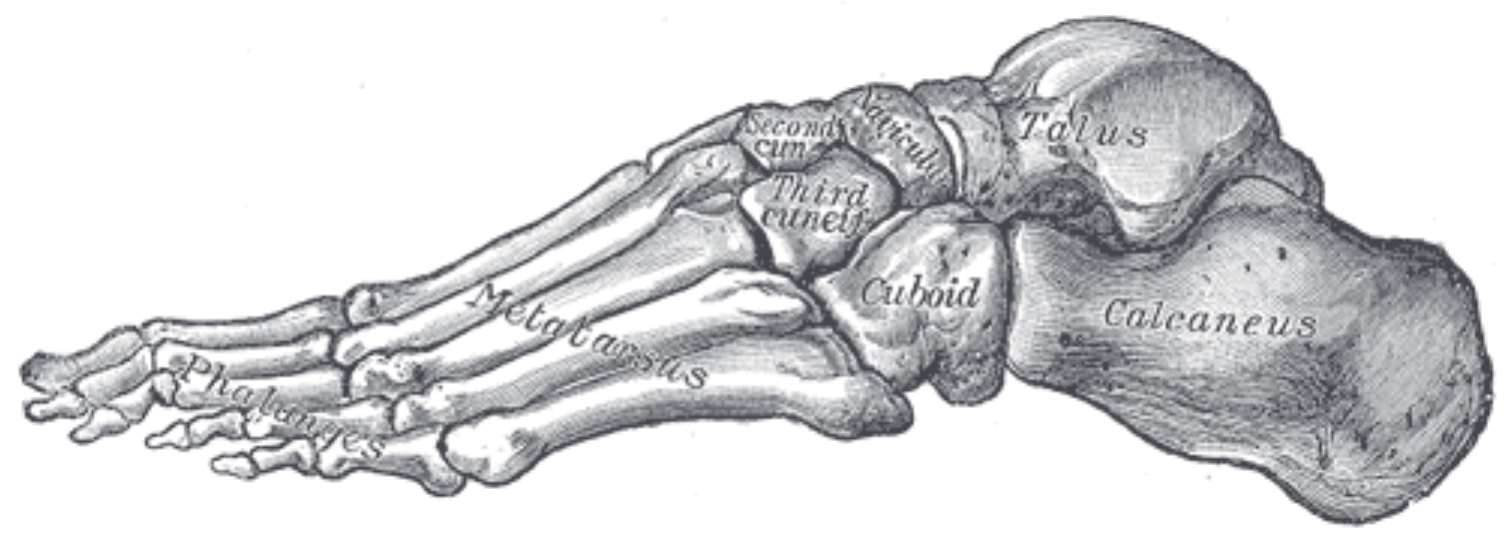

Figure 1: Bony anatomy of the foot: Tarsal bones include navicular, cuboid, and cuneiforms. Metatarsals and phalanges are numbered one through five starting medially then ending laterally (Gray, 1918).

In order for the foot and ankle to move, the bones must work together through joint contact. Some of the most important joints for movement in the foot and ankle are the tibiotalar joint, subtalar joint, transverse tarsal joint, tarsometarsal joints, and metatarsophalangeal joints (Prior, 1999). The tibiotalar joint is located where the tibia 
meets the talus, forming a trapezoidal shape from the medial malleolus, the talar plafond as the center, to the lateral malleolus (Scranton, McMaster, \& Kelly, 1976; Schriebman \& Bruce, 2009). Stability of the ankle joint is formed by this trapezoidal shape, which provides a wide base for a large distribution of force (Scranton, McMaster, \& Kelly, 1976; Schriebman \& Bruce, 2009). Because the tibiotalar joint connects leg to foot, a sturdy base is needed as body weight is distributed from the upper body to the foot. The subtalar joint is located below the talus (hence the name "subtalar") where the calcaneus articulates with the talus (Figure 2). The mid-tarsal joint combines two joint processes into one term, because both joints are in the middle of the foot in the tarsal region. One of the joints that is part of the mid-tarsal joint is the talonavicular joint, located between the talus and the navicular bone (Figure 2). The other mid-tarsal joint is the calcaneocuboid joint at the junction of the calcaneus and cuboid (Figure 2). Both the subtalar joint and mid-tarsal joint are interlinked and allow for three-planar motion, which is vital to healthy foot function (Prior, 1999). The tarsometatarsal joints are located between the tarsal bones (cuneiforms and navicular) and the metatarsals. The metatarsophalangeal joints are located between the metatarsals and the phalanges associated with motion of the toes. 

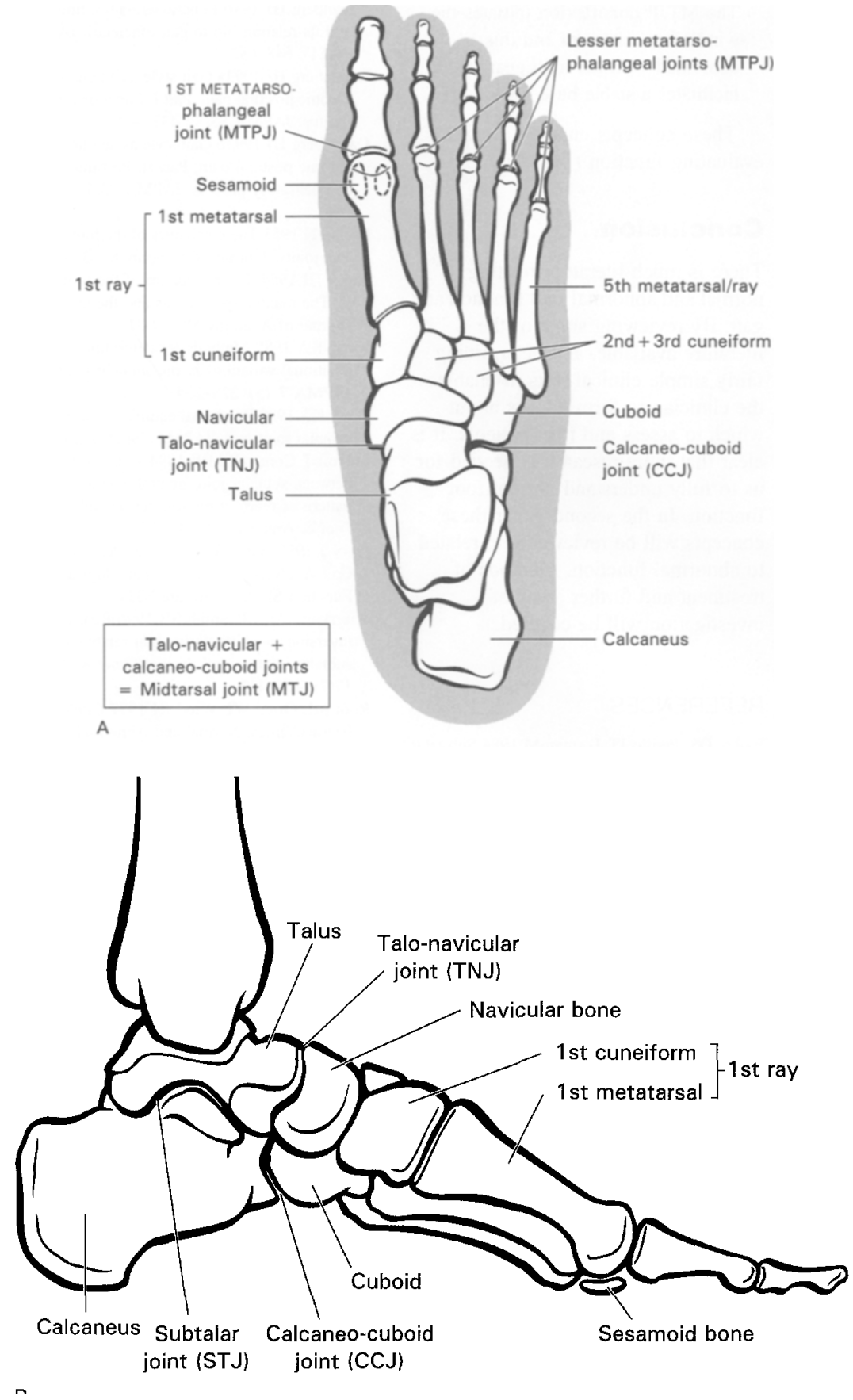

Figure 2: A: an anteroposterior view of the foot including joint structures and bones. B: a mediolateral view of the foot bones including joint structures (Prior, 1999).

To orient the foot about certain axes the movements described are as follows:

plantarflexion and dorsiflexion rotate about a transverse axis, pronation and supination 
rotate about an anteroposterior axis, and finally, internal rotation and external rotation (Prior, 1999) (Prior, 1999)rotate about a vertical axis (Figure 3) (Hicks, 1953). The tibiotalar joint has plantar flexion and dorsiflexion axes. The plantarflexion axis can be found anterior and inferior to the tip of the medial malleolus and superior to the tip of the lateral malleolus (Hicks, 1953). The dorsiflexion axis can be found anterior to the tip of the medial malleolus and inferior to the tip of the lateral malleolus (Figure 4) (Hicks, 1953). Due to differences in bone shape, there may be a significant difference in axis location from person to person. The subtalar joint provides for the axis of rotation of both the foot and ankle in various movements such as pronation, supination, internal rotation, and external rotation. Subtalar motion is the predicator to potential issues that may occur in the foot because it the bridges the connection between leg and foot (Klenerman \& Wood, 2006).

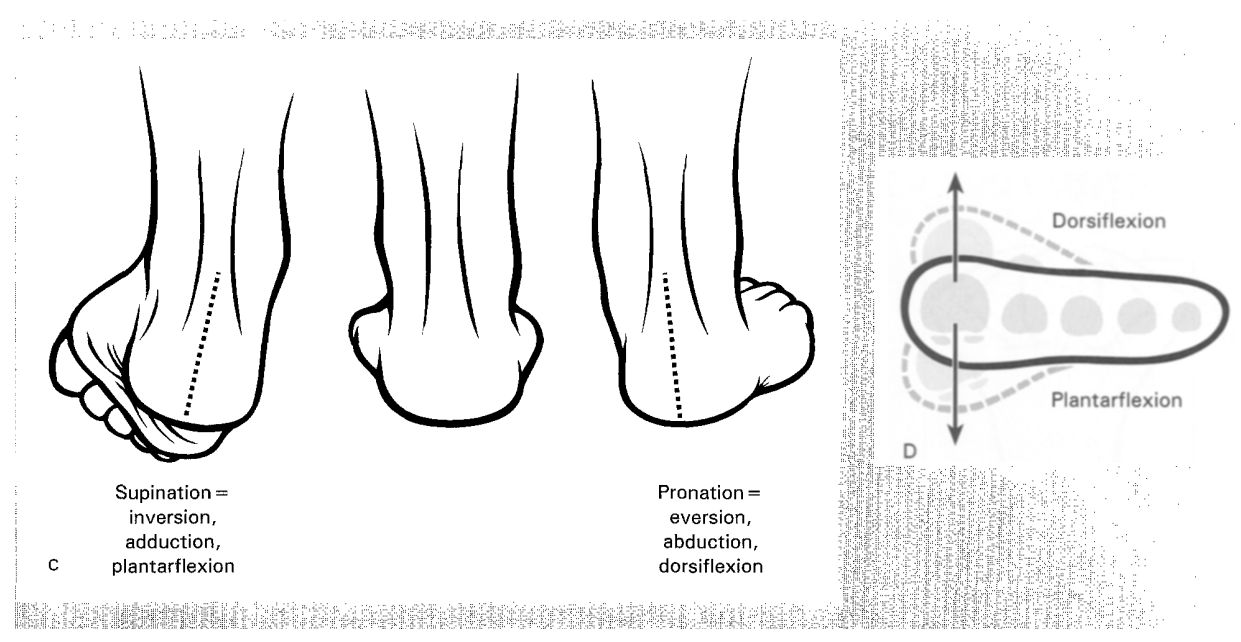

Figure 3: Showing axes of rotation and relative movements of the foot and ankle that cover supination and pronation of the foot (Prior, 1999) 




Figure 4: Plantarflexion (blue) and Dorsiflexion (red) axis in the tibiobtalar joint of the ankle (Hicks, 1953)

The subtalar joint articulates at three different sites along the talus and calcaneus at the sustentaculum tali, anterior portion of the head of the talus that rests on the anterior process of the calcaneus, and the posterior portion of the talus to the middle of the calcaneus (Schriebman \& Bruce, 2009). The action of the subtalar joint has frontal plane motion, sagittal plane motion, and transverse plane motion (Klenerman \& Wood, 2006) where supination and pronation occur (Figure 3). About a single axis of rotation, the subtalar joint is restricted during inversion due to the axis of the talonavicular and calcaneocuboid joints not being parallel to each other (Elftman, 1960) (Wheeless III, Nunley II, \& Urbaniak, 2011). This change in angle in the midfoot stablizes the foot so that it can act as a lever during toe off to propel the person forward (Elftman, 1960). The opposite occurs for eversion, where the talonavicular and calcaneocuboid joints are parallel (Wheeless III, Nunley II, \& Urbaniak, 2011). During gait, inversion locks the 
midtarsal joints so that the foot is rigid at toe off, while eversion allows for maximal motion at midtarsal joints to absorb energy at heel strike (Elftman, 1960) (Wheeless III, Nunley II, \& Urbaniak, 2011). This joint can also be called the talo-calcaneao-navicular joint, where the navicular is also added into the subtalar joint (calcaneus and talus) only articulating with the talus. The axis for this joint can be found on the supero-medial aspect of the neck of the talus to the postero-lateral "corner" of the heel (Figure 5) (Hicks, 1953).

There are other structures that dictate foot and ankle movement including the midtarsal joint. The midtarsal joint is actually two joints, the talonavicular and calcaneocuboid joints (Klenerman \& Wood, 2006). Although it is two joints, the movement between the cuboid and navicular bones is very minor where the two bones move as one, therefore they are considered one "joint" (Klenerman \& Wood, 2006). There are two axes in the joint that describe its motion; one is the oblique axis that is located at the supero-medial aspect of the head of the talus to the lateral side of the inferior surface of the heel, anterior to talo-calcaneo-navicular joint (Figure5) (Hicks, 1953). The other axis of the midtarsal joint is the antero-posterior axis that is at the superior aspect of the navicular between the midline of the dorsum of the foot and is superior to the talo-calcaneo-navicular axis (Figure 5) (Hicks, 1953). All three axes pass through the head of the talus at the center sphere where the control of side-to-side balance is present (Hicks, 1953). The three axes are important with supination, pronation, walking on an incline, and side-to-side motion (Hicks, 1953). For those with an injury in the ankle, the movements involved with the three axes may be restricted or unattainable, especially in the talo-calcaneo-navicular axis. It has been shown that those with an issue 
of movement in the talo-calcaneo-navicular axis are unable to maintain side-to side balance during single leg stance (Hicks, 1953).

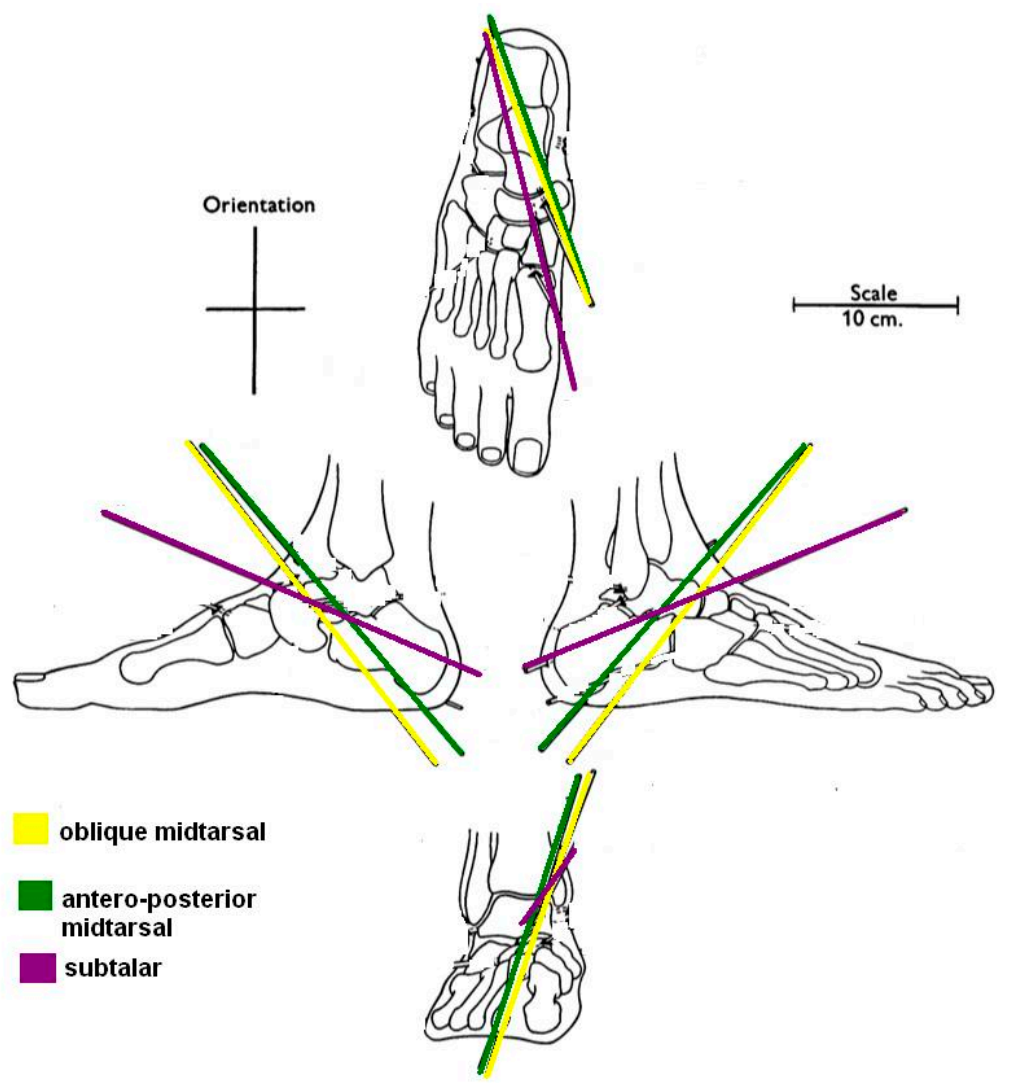

Figure 5: The talo-calcaneo-navicular (subtalar) axis (green), Oblique midtarsal axis (yellow) and antero-posterior midtarsal axis (purple) shown in different views (Hicks, 1953).

Since the foot is the support structure that humans put all of their weight on, it is vital to distribute the large loads properly. Morphologically the load is spread across multiple bones in the foot, however if these bones are malformed or moved from normal anatomical position, the load will most likely fall on structures that are not capable of handling large forces. Soft tissue structures and cartilage help facilitate load distribution. Cartilage is present between the bones, which allow changes in the elasticity of the joint structure and shock absorption with jarring movements (Klenerman \& Wood, 2006). 
Running and jumping without bone breakage is made possible through cartilage shock absorption between bones.

Additional soft tissue structures provide stability, structure and allow movement in the foot. Tendons are the connectors from bones to muscles back to bones whereas ligaments connect the bone-to-bone (Fung, 1993). Muscles are the soft tissue structures that are arranged to absorb force as well as create it through contraction with the lowest energy expenditure under ideal conditions (Fung, 1993). Tendons, ligaments and muscles in the foot are also flexible enough to permit motion without causing or incurring injury. Stretching of soft tissue structures is normal; however, overstretching can cause injury. Most soft tissue structures have a high elastic modulus with tendons at $700 \mathrm{MPa}$, collagen at $500 \mathrm{MPa}$, and muscle fascia at $350 \mathrm{MPa}$ (Palsson \& Bhatia, 2004).

In the foot, the soft tissue structures are not only important to foot and ankle movement but also to the configuration of the arch. The medial arch is made up of the medial bones that stretch from posterior calcaneus, talus, navicular, three cuneiforms, to the metatarsal heads 1,2,and 3 (Gray, 1918). Elasticity of the medial arch is vital to the correct biomechanical functioning from the number of joints and height from the ground where structures such as the flexor hallux longus, provide stability (Gray, 1918). The weakest part of the arch is at the junction of the navicular and talus (Gray, 1918). Supporting this weak spot, plantar calcaneonavicular ligament and posterior tibial tendon stretch when force is applied and return to normal once the force is removed (Figure 6) (Gray, 1918). Due to its fan-like structure, the posterior tibial tendon plays a key role in force distribution for the plantar calcaneonavicular ligament, so that overstretching does not occur (Gray, 1918). The posterior tibial tendon sustains large forces and protects the 
plantar calcaneonavicular ligament from damage; therefore, if the posterior tibial tendon is compromised then the arch will begin to collapse and cause overstretching of structures.

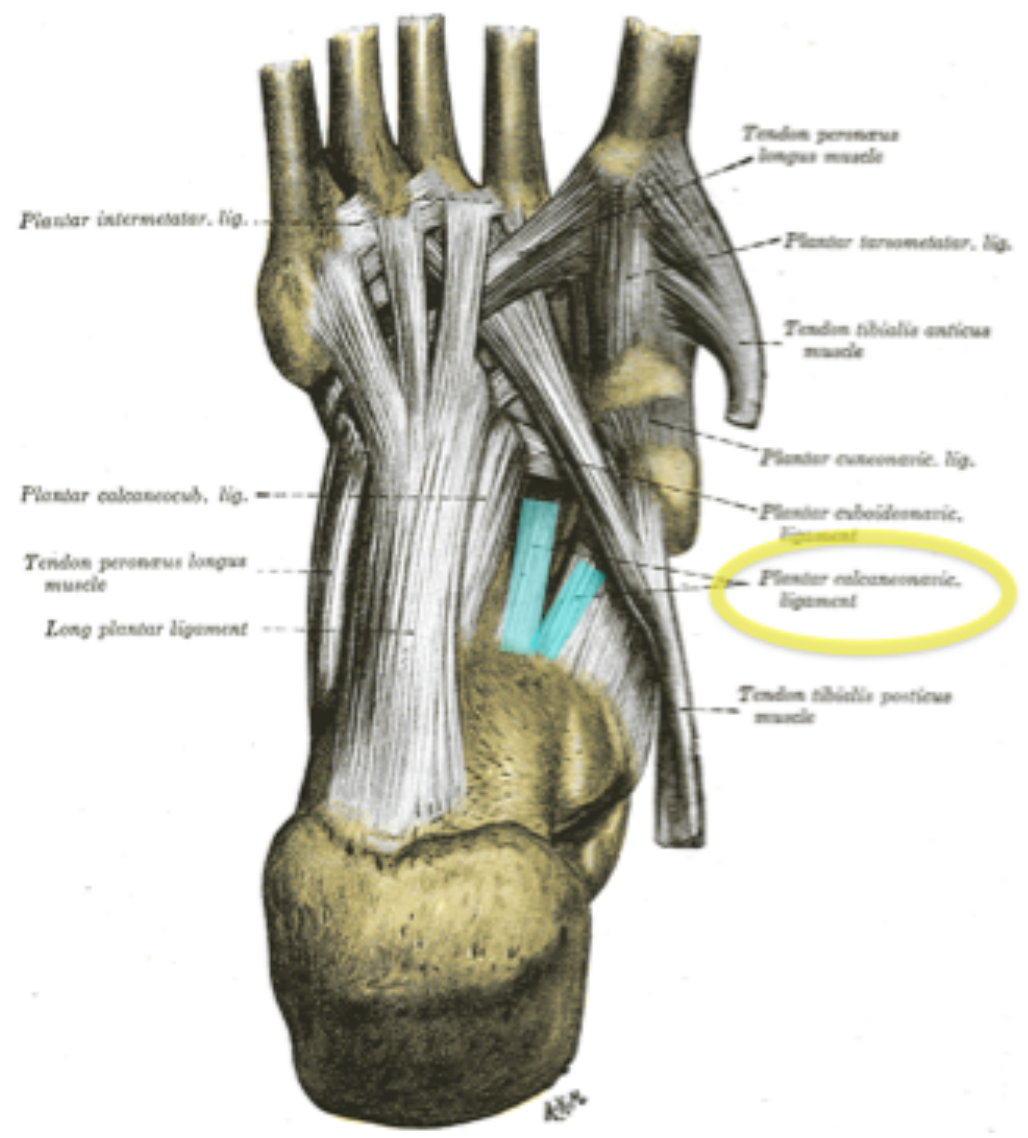

Figure 6: Ligamenture of the foot from an underside view of the foot and the calcaneonavicular ligament/spring ligament complex (Gray, 1918).

The posterior tibial tendon extends from above the medial malleolus to the navicular on the medial side of the ankle (Figure 7) (Gray, 1918). As seen in Figure 7, the posterior tibial tendon originates from the posterior surface of the interosseous membrance and adjacent posterior surfaces of tibia and fibula and then inserts into the tuberosity of the navicular (McMinn, Hutchings, \& Logan, 1996). The functions of the 
posterior tibial tendon are to plantarflex and invert the foot acting as the main supinator during the stance (McMinn, Hutchings, \& Logan, 1996).



Figure 7: The medial ankle showing the posterior tibial tendon and other facia around the ankle (Gray, 1918).

The spring ligament complex plays an important role in keeping the medial arch structure intact. The spring ligament complex is made up of multiple ligaments that include the superomedial ligament and the inferior calcaneonavicular ligament that create a triangular shape from the sustentaculum tali and attach to the navicular bone (Figure 6) (Deland J. , 2001) (Jennings \& Christensen, 2008) (Rule, Yao, \& Seeger, 1993). Anatomically, the spring ligament runs superficial to the posterior tibial tendon that provides some support to the spring ligament (Rule, Yao, \& Seeger, 1993). Some descriptions include a third portion of the ligament complex, the plantar calcaneonavicular ligament (Figure 6) (Durrant, Chokalingham, \& Hashmi, 2011) (Deland J. , 2001). Although some anatomical discrepancies in what the spring ligament 
complex entails, biomechanically it is agreed that the spring ligament complex provides stability in the mediolongitudinal arch and the subtalar joint by supporting the head of the talus (Durrant, Chokalingham, \& Hashmi, 2011) (Rule, Yao, \& Seeger, 1993) (Van Boerum \& Sangeorzan, 2003).

The plantar fascia (plantar aponeurosis) is the insertion of the plantaris muscle that spans across the sole of the foot (Figure 8) (Klenerman \& Wood, 2006). The fibers of the muscle are longitudinal and start at the calcaneus fanning to each digit; making this structure a stronghold for the longitudinal part of the medial arch (Gray, 1918). This muscle is extremely important during gait as it distributes a large load across the foot because of the long structure spanning the entire foot. As seen in Figure 9, this structure contributes to raise the longitudinal arch when toes are dorsiflexed where the largest effect is on the first ray (both the metarsophalangeal and digit as one single structure), which then decreases laterally (Klenerman \& Wood, 2006). It can be assumed that there is the opposite effect when the toes are plantarflexed where the arch is flattened (Klenerman \& Wood, 2006). The mechanics of the plantar fascia have shown that the structure elongates before midstance of gait and also immediately after (Figure 9) (Klenerman \& Wood, 2006). During midstance, the maxiumum amount of force on the foot is present, therefore it is imperative that the arch be stabilized and prepared. Elongation stabilizes the arch complex by providing an opposing calcaneal force while allowing for the foot to support the arch structure during the most critical times in gait (Wheeless III, Nunley II, \& Urbaniak, 2011) (Shraeder \& Lohmann Siegel, 2003). 


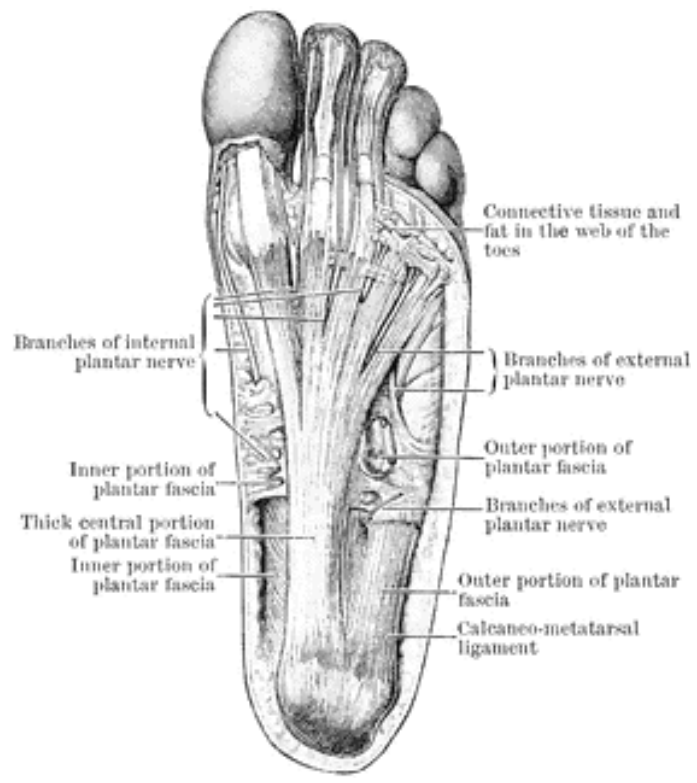

Figure 8: Plantar aperneurosis (plantar fascia) extending from the calcaneus to the digits as it supports the longitudinal arch of the foot (Gray, 1918).

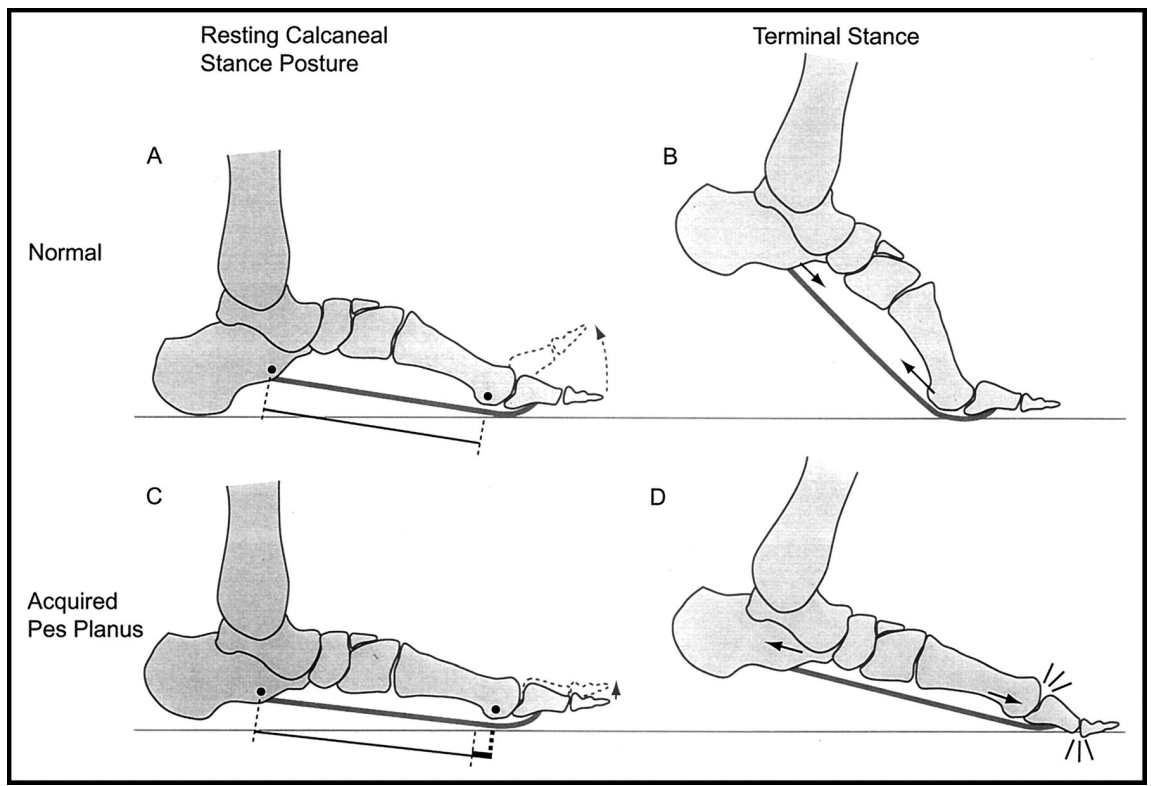

Figure 9: The effect of dorsiflexing the foot on the arch (A) and the effect of walking with the change in arch height due to the plantar fascia (B). Flatfoot lengthens (C) and does not allow for opposing forces on terminal stance (D) (Shraeder \& Lohmann Siegel, 2003) 


\section{DEFINING ADULT-ACQUIRED FLATFOOT DEFORMITY}

Adult acquired flatfoot deformity (AAFD) is a progressive disease that is defined by the mechanical degeneration of soft tissue structures, which cause changes in joint alignment of the bones of the foot. There are many soft tissues that are affected that primarily include the posterior tibial tendon, spring ligament, talocalcaneal interosseous ligaments, and the long and short plantar ligaments (Deland, de Asla, Sung, Ernberg, \& Potter, 2005). Clinically, the alterations in soft-tissue structure are seen as midfoot collapse, forefoot abduction and valgus tilting in the hindfoot (Deland, de Asla, Sung, Ernberg, \& Potter, 2005) (Blackman A. , Blevins, Sangeorzan, \& and Ledoux, 2009) (Hill, Saar, Lee, \& Berlet, 2003) (Kitaoka, Ahn, Luo, \& An, 1997). The degradation process determines the severity of the disorder, defined in stages, with the progression to full failure of soft-tissue structures. In the early stages, the medial ankle will have swelling and pain over the posterior tibial tendon (Deland J. T., 2008). Although complete failure of tissue is rare, the painful symptoms that accompany this affliction are enough to require surgical intervention as the deterioration increases.

The posterior tibial tendon degrades over time, which is an integral part of defining the cause of anatomical flatfoot (Jennings \& Christensen, 2008). The complex etiology of flatfoot and the change in soft tissue structures makes it difficult to assess the exact trigger of the dysfunction; however, the posterior tibial tendon is a key player in adult acquired flatfoot deformity (AAFD). When the posterior tibial tendon becomes dysfunctional, it is assumed that the toes will not be able to push off with as much force during toe-off at gait due to lessening of plantarflexion. Inversion will not be as prevalent 
during the stance phase of gait, as well as pure stance, therefore there is a drop in the medial midfoot because the posterior tibial tendon is compromised. The forefoot becomes longer because as the arch collapses the foot straightens (Van Boerum \& Sangeorzan, 2003). As the arch collapses, the calcaneus subluxates posteriorly without supporting the talar head anteriorly (Van Boerum \& Sangeorzan, 2003). As the posterior tibial tendon degrades symptoms will worsen, which will make it difficult to walk and continue with normal activities of daily living.

Although more investigation is needed, the spring ligament complex is closely linked to the failure of the posterior tibial tendon (Jennings \& Christensen, 2008) (Van Boerum \& Sangeorzan, 2003) (Durrant, Chokalingham, \& Hashmi, 2011) (Deland J. , 2001). Typically with the diagnosis of posterior tibial tendon insufficiency there is a lesion in the spring ligament complex because it is the weakest part of the medial arch (Society, 2010) (Jennings \& Christensen, 2008) (Deland J. , 2001) (Durrant,

Chokalingham, \& Hashmi, 2011) (Van Boerum \& Sangeorzan, 2003; Gray, 1918). It has been suggested that the spring ligament is the primary cause of the development of flatfoot, and posterior tibial tendon insufficiency is a result of the spring ligament failure (Jennings \& Christensen, 2008). Regardless of whether the deficiency is a primary or secondary role to the posterior tibial tendon, the spring ligament complex is closely related to the flatfoot affliction (Deland J. , 2001) (Society, 2010) (Durrant, Chokalingham, \& Hashmi, 2011).

\section{CHARACTERISTICS OF THE AFFLICTION}

Clinical characteristics of this disorder are based upon the radiological 
measurements, as well as qualitative clinical tests. A person that is affected with flatfoot deformity would have an onset of pain and pressure in the medial portion of the ankle toward the medial malleolus (Deland J. T., 2008). As the disease progresses the pain may subside however, swelling usually is still apparent with the possibility of ligament and tendon failure (Deland J. T., 2008). Pain is not a good indicator of the severity of the flatfoot condition as it is not linear; most patients vary on the pain level scale during different stages.

Although there are many factors that affect the progression of flatfoot, several characteristics place individuals more at risk. As mentioned, the area where flatfoot arises is mechanically the weakest portion of the arch. Morphologically, this same segment contains soft tissue structures that are hypovascular (Holmes GB Jr, 1992). Blood flow allows for the healing process to continue with oxygenated blood helping to bring nutrients to the area and take away waste products. If the area has hypovascular properties then the injured tendon may not be able to heal as easily, leading to degeneration (Van Boerum \& Sangeorzan, 2003). Other non-anatomical factors include race, age, body mass, and sex.

Race is a determining characteristic of flatfoot where African Americans are more at risk. According to a study that characterized foot conditions in older adults (65 years or older) of over 750 people, the significant finding was flatfoot prevalence in African American individuals (Dunn J.E., 2004). Calcaneal pitch, which is the angle of the calcaneus to the floor, is significantly lower in African Americans than non-Hispanic Whites and Hispanics (Castro-Aragon, 2009). Calcaneal pitch is a measure of flatfoot where the lower the angle, the more flat the foot is. Hispanics are also more predisposed 
to flatfoot than non-Hispanic Whites, but there is a significant difference between African-Americans and the two other populations (Castro-Aragon, 2009). Since AfricanAmericans were shown to have the lowest calcaneal pitch, it can be concluded that they may be morphologically predisposed to flatfoot over other ethnicities.

Aging is another risk factor for flatfoot deformity that is associated with overuse and increased amount of time spent on afflicted foot. Age-related changes in foot structure are due to appearance of different musculoskeletal deformities. For example, hallux valgus where the first toe is turned laterally is an indicator of flatfoot and common among older adults (Scott G, 2007). Arch height, represented by the height of the navicular bone to the floor, is significantly smaller in older adults versus young adults (Scott G, 2007) (Faria, Gabriel, Abrantes, Bras, \& Moreira, 2010). Smaller navicular height means that the foot is more flat because the navicular should be raised off the ground to be anatomically normal.

Although there is no conclusive evidence as to why women are afflicted more than men, this may be due to hormonal changes in post-menopausal women (Richie, 2007). Lower arches require more control of the soft tissue structures of the foot due to unequal distribution of force. (Faria, Gabriel, Abrantes, Bras, \& Moreira, 2010) Post-menopausal women have lowered stiffness in ligamenture, which makes the arch more flexible and less controlled (Faria, Gabriel, Abrantes, Bras, \& Moreira, 2010). A more flexible arch causes more stretching in the soft tissue, escalating the demand of soft tissue structures ultimately creating a foundation for injury. Hormonal and metabolism changes may also increase the readiness of the body to put on extra weight, leading to extra force on the foot and ankle. 
An overweight person may be at risk for flatfoot due to the increased loads experienced by joints and soft tissue structures of the foot (Blackman A., Blevins, Sangeorzan, \& Ledoux, 2009) (Faria, Gabriel, Abrantes, Bras, \& Moreira, 2010). According the modulus of the specific tendon or ligament, the overstretching and failure points are crucial in determining how much stress and strain the tissue can handle. As soft tissues are overstretched, the biomechanical properties (particularly stiffness) degrade making them more susceptible to failure. Specifically, the calcaneonavicular ligament is overstretched when the posterior tibial tendon to bears more of the load. Because the posterior tibial tendon has multiple insertion sites, the tendon can produce a high force with minimal movement due to the anchoring at insertion sites (Hintermann, 1994), however when AAFD is diagnosed, the force is reduced greatly.

\section{STAGES OF FLATFOOT}

Stages of flatfoot deformity range from I to IV where each stage is characterized by the severity of the condition. Measuring the joint angles and flexibility of the foot identifies each different stage. Eventually the stages progress where the foot becomes less flexible as the posterior tibial tendon lengthens, soft tissue structures stretch and joint alignment changes.

Stage $\mathrm{I}$ is a pre-existing condition that does not arise from insufficiency of the posterior tibial tendon (Deland J. T., 2008; Johnson K.A, 1989). This condition may be due to genetics or previous injury that would cause a flatfoot deformity. The symptoms of this stage are usually general discomfort, tenosynovitis or tendinosis (Deland J. T., 2008). This stage is considered least severe and is usually treated with conservative measures 
such as immobilization and bracing of the foot to stabilize. Surgical intervention does not usually proceed with this particular stage because most patients respond to conservative treatment. In Stage I flatfoot the posterior tendon does not become insufficient over time.

The foot is less flexible where a moderate flexible deformity occurs with exposing the talar head between the joint of the talus and navicular. The articulation with the navicular, the talus sits in a rounded groove for a normal foot, but moves medially with the progression of flatfoot (Figure 10). There are two forms of this stage that vary by levels of severity and the extent of talonavicular uncoverage. Stage IIa is described as a mild/moderate flexible deformity where there is minimal abduction through the talonavicular joint with less than thirty percent uncoverage (Johnson K.A, 1989; Deland J. T., 2008). Stage IIb is described as a severe flexible deformity where the abduction through the talonavicular joint is now deformed with more than thirty percent talonavicular uncoverage (Johnson K.A, 1989; Deland J. T., 2008). Stage II is considered to be a flexible deformity because the patient is still able to walk, but it is much more painful because the alignment of the foot has changed. Foot alignment can be corrected through bracing, by passively inverting the talonavicular joint and correcting the heel placement to match with normal anatomical parameters (Deland JT, 2006). Although braces may limit ankle motion, they are a good alternative to place the ankle in correct position and stabilize a weak foot (Deland J. T., 2008). Bracing the ankle is a conservative treatment to a surgical alternative; however, if symptoms persist, surgery may be a necessary. This stage is considered to be the most controversial to operate because of the debate between range of motion and functionality to stabilize the arch. 




Figure 10: An AP view of the foot bones where the talonavicular joint is highlighted (Gray, 1918). The arrow shows the direction of the movement of the talus when "uncovering" the head during Stage II of flatfoot deformity.

A fixed deformity occurs in Stage III where passive inversion that could be done in Stage II cannot be performed. The "triple joint complex" composed of the talonavicular, subtalar, and calcaneocuboid joint does not allow movement beyond the neutral plantigrade position (Deland J. T., 2008). Other symptoms include abduction through the midfoot and a fixed hindfoot valgus (Deland J. T., 2008). The treatment for this severe condition is a triple arthrodesis, which requires the fusing of all three joints together (Deland J. T., 2008). This limits the foot's natural range of motion especially when walking, in comparison to earlier stages (Deland JT, 2006). The patient is typically limited in activities of daily living where surgery is required to correct alignment. The 
foot is deformed to the point where no passive measures can be taken.

Stage IV flatfoot deformity is divided into sub-stages due to the difference in the foot deformity, however both share an ankle deformity as well. The main distinction between other stages and Stage IV is the addition of ankle deformity. A lateral talar tilt, usually found radiographically, which indicates deltoid ligament failure, is the common deformity in the ankle (Deland J. T., 2008). Stage IVa is a flexible foot deformity where the foot can passively be realigned like in Stage II (Deland J. T., 2008). In comparison to Stage III, the more common deformity is the foot to be a fixed deformity as seen in Stage IVb with the addition of deformity of the ankle (Deland J. T., 2008). Surgical intervention for Stage IV is inevitable since both the foot and ankle need to be corrected. Once the ankle is corrected it is up to the physician to surgically correct the foot depending on the fixed or flexible nature of the foot (Deland J. T., 2008). It is unlikely that a patient will get to this point of deformity as it is extremely painful and the patient will be unable to walk.

\section{DIAGNOSIS OF FLATFOOT}

There are many different measurements that are a part of a positive flatfoot diagnosis, both qualitative and quantitative. Although there are no gold standards for measuring flat foot deformities, there are some clinical and radiographic measurements felt to be helpful in diagnosing a patient with flatfoot (Lee K. M., 2010). The physical exam is the basis for the qualitative diagnosis where the patient is asked to stand on one foot and lift the heel off the ground. This is considered the "heel rise" test where a patient with AAFD should either have extreme difficulty performing this, or may not be able to perform at all (Deland J. T., 2008) (Lee D. , 2009). At the point of heel rise, the force on 
the weakest part of the arch is peaked; therefore, if the soft tissue structures are compromised then this task would be almost impossible. This test may have some pitfalls due to false positive test results from other existing conditions such as arthritis, a fusion of joints or Achilles rupture (Deland J. T., 2008).

Another clinical test that is used is obtained from behind with knees pointing forward and the foot standing flat. A goniometer or visual cues may be used to determine hindfoot valgus as seen in Figure 11 (Haight, Dahm, Smith, \& Krause, 2005). Also, a visual sign of "too many toes" is an indication that a person has a flatfoot deformity, where the lesser toes may show from a hindfoot view (Lee K. M., 2010) (Coughlin \& Kaz, 2009). A dropped arch is seen qualitatively in flatfoot patients, where the soft tissue structure laxity moves the arch bones lower to the ground, if not all the way flush. Some physicians diagnose flatfoot just by using the qualitative view of the hindfoot where the toes and the dropped arch is a good indicator as to how flat the foot is (Coughlin \& Kaz, 2009) (Lee D. , 2009). To quantify the severity of subtalar joint movement, a goniometer is used to measure the angle using the long axis of the Achilles, the talus, and the axis of the calcaneus as landmarks (Coughlin \& Kaz, 2009) (Haight, Dahm, Smith, \& Krause, 2005) (Gluck, Heckman, \& Selene, 2010). There are concerns that although hindfoot angle may be a great measure of flatfoot, the subtalar joint is not horizontal therefore the valgus would not occur in one simple plane (Lee K. M., 2010). Because the subtalar joint affects more than one plane the resultant angle that is measured may not be comparable from patient to patient. 


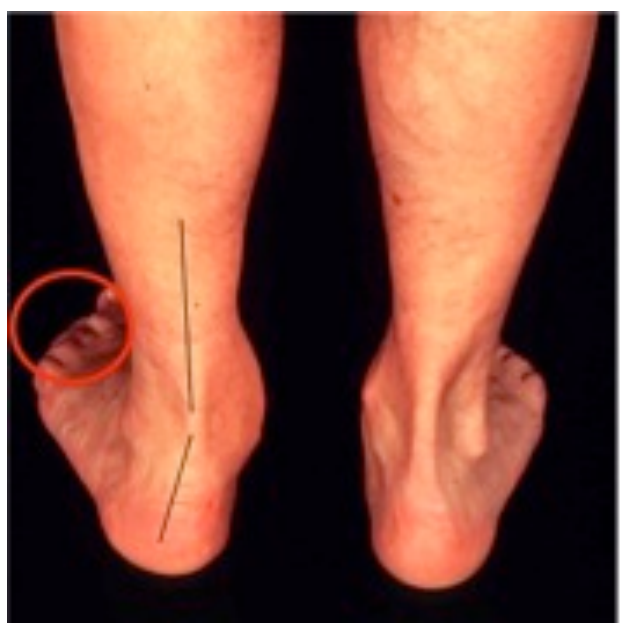

Figure 11: Hindfoot view of normal (right) and afflicted foot (left) where heel valgus(black lines) and signs of "too many toes" occurs (red circle) www.orthosportssg. com/?feed $=$ rss $2 \&$ cat $=5$

\section{SURGICAL DEBATE}

There is much controversy as to how to treat AAFD especially the more flexible stages. Stage I is treated conservatively, preferably with non-operative measures with the use of orthotics and bracing (Deland J. T., 2008) (McCormack, Ching, \& Sangeorzan, 2001), which is widely accepted throughout the clinical community. Stage IIa and IIb are a bit more difficult to treat because surgical intervention is necessary in order to correct the disorder. The question arises: how can the flexibility of the joints be preserved while the flatfoot is corrected?

Surgeons have different preferences in the procedures to correct flatfoot, either surgically or non-surgically, depending on factors such as activity level, age, and the severity of the deformity (McCormack, Ching, \& Sangeorzan, 2001). All factors should be considered, using surgical intervention as a last resort. Although many techniques have been used such as debridement with augmentation of the posterior tibial tendon, modified Hoke-Miller procedure where both bony and soft tissue are corrected, spring ligament reconstruction, lateral column lengthening, tenodesis, tendon transfer, 
osteotomy, and arthrodesis (McCormack, Ching, \& Sangeorzan, 2001) (Greisberg, Assal, Hansen, \& Sangeorzan, 2005). Some of these procedures have been used alone but the most effective surgeries to correct flatfoot are a combination of the techniques that are tailored to the specific symptoms of the patient (McCormack, Ching, \& Sangeorzan, 2001).

Using an approach to correct both soft tissue and bony tissue helps to preserve some of the flexibility of the foot. Procedures using a medial calcaneal osteotomy (MCO) as well as a tendon transfer of either the flexor hallucis longus (FHL) or flexor digitorum longus (FDL) try to preserve the normal motion of the ankle joint while preserving the function of the toes (Arangio \& Salathe, 2009) (Richardson, Willers, Cohen, Davis, Jones, \& Anderson, 2009). Other procedures correct the spring ligament as well as the dysfunctional posterior tibial tendon (Jennings \& Christensen, 2008) (Deland J. , 2001) (Society, 2010). Because these surgical interventions require the manipulation of the soft tissue, some argue that the patient is more likely to return with future flatfoot issues and thus prefer to fuse joints together instead (Deland J. T., 2008). This is the common technique for later stages and fixes the biomechanical failure in the arch, however it does not leave the patient with much flexibility in the ankle joint (Deland J. T., 2008). Fusion provides more stability for the flatfoot and less of a possibility for change since the surgery fixes the bony structure together and does not rely upon soft tissue to heal and be placed properly.

\section{RADIOGRAPHIC MEASUREMENTS}

Clinical diagnosis of AAFD is mainly made upon both the qualitative and quantitative nature of radiographs. Unfortunately, since no set protocol has been 
determined to measure the angles in a radiograph it is important to understand the common measures that are used to determine flatfoot. Both AP and ML radiographs are used in determining the quantitative angular measures of the foot.

\section{CLINICAL USAGE OF RADIOGRAPHS}

Radiographic measurements are used to quantify flatfoot severity and can be readily obtained clinically. The bones that are most important for radiographic diagnosis of flatfoot are the navicular, talus, first metatarsal, and calcaneus. These bones are particularly affected when the arch collapses and thus relative movement are indicative of flatfoot. The navicular bone is crucial to determining the arch height of the foot due to placement and mechanical forces applied when bodyweight is added. The talonavicular joint uncoverage is crucial to determining the stage of flatfoot as the disorder progresses, the more abduction of the foot occurs (Johnson K.A, 1989; Deland J. T., 2008). The angle of the calcaneus to the ground (calcaneal pitch) shows qualitatively how flat the foot is, and is used to diagnose the condition (Karasick \& Schweitzer, 1993) (Coughlin \& Kaz, 2009) (Murley, Menz, \& Landorf, 2009) (Lee K. M., 2010)

Both anteroposterior (AP) and mediolateral (ML) views are obtained to determine key angles indicative of flatfoot. Qualitatively it can be seen in the mediolateral (ML) view (Figure 12) that severe cases the entire joint collapses due to lack of soft tissue structural elements (Van Boerum \& Sangeorzan, 2003) The AP radiograph (Figure 13) shows the abduction through the talonavicular joint where the first metatarsal angle can be used to show the abnormality. 

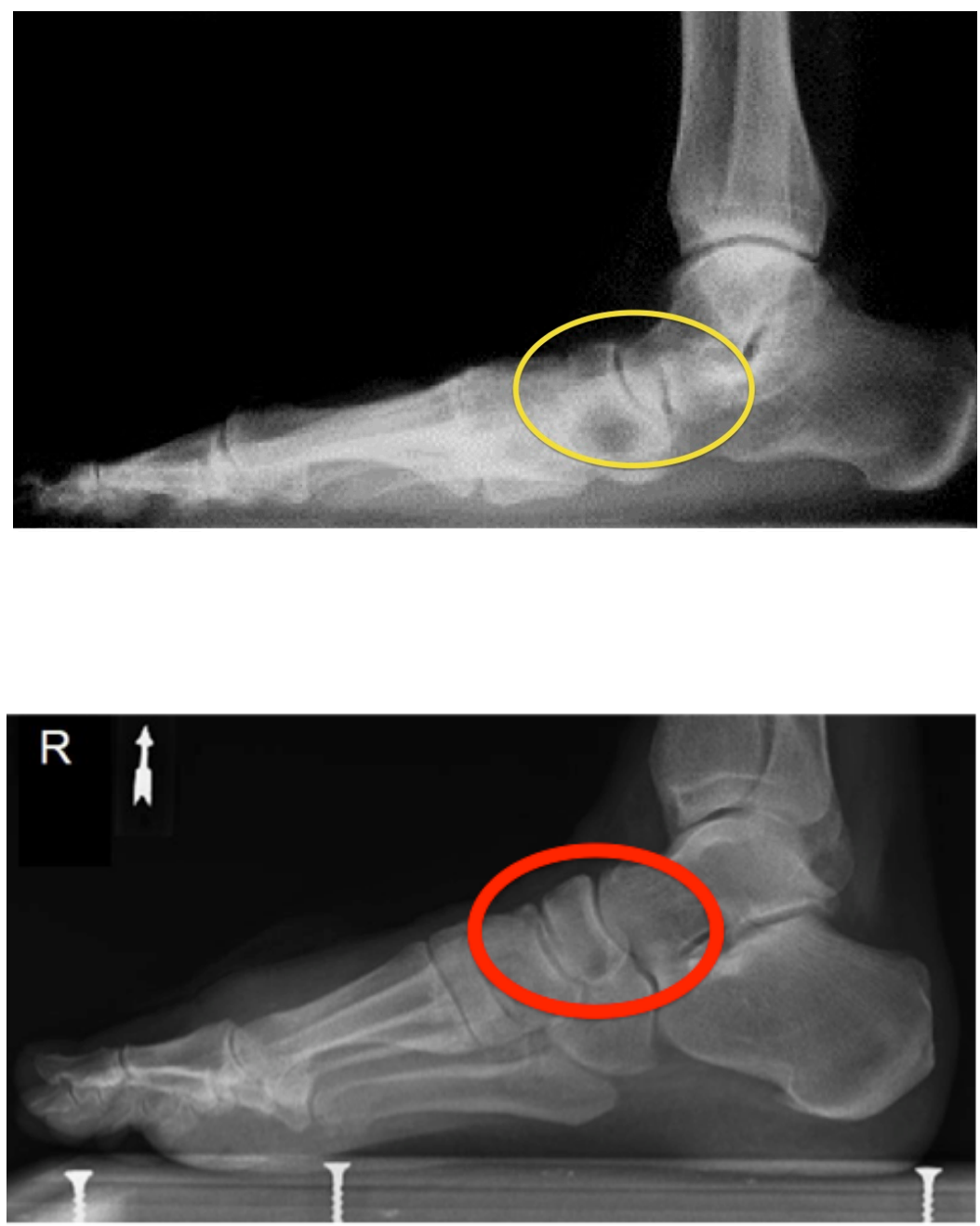

Figure 12: ML Radiograph: Showing talonavicular collapse highlighted in yellow (Van Boerum \& Sangeorzan, 2003) and normal radiograph showing arch structure in red (Joseph Jr., 2008) 

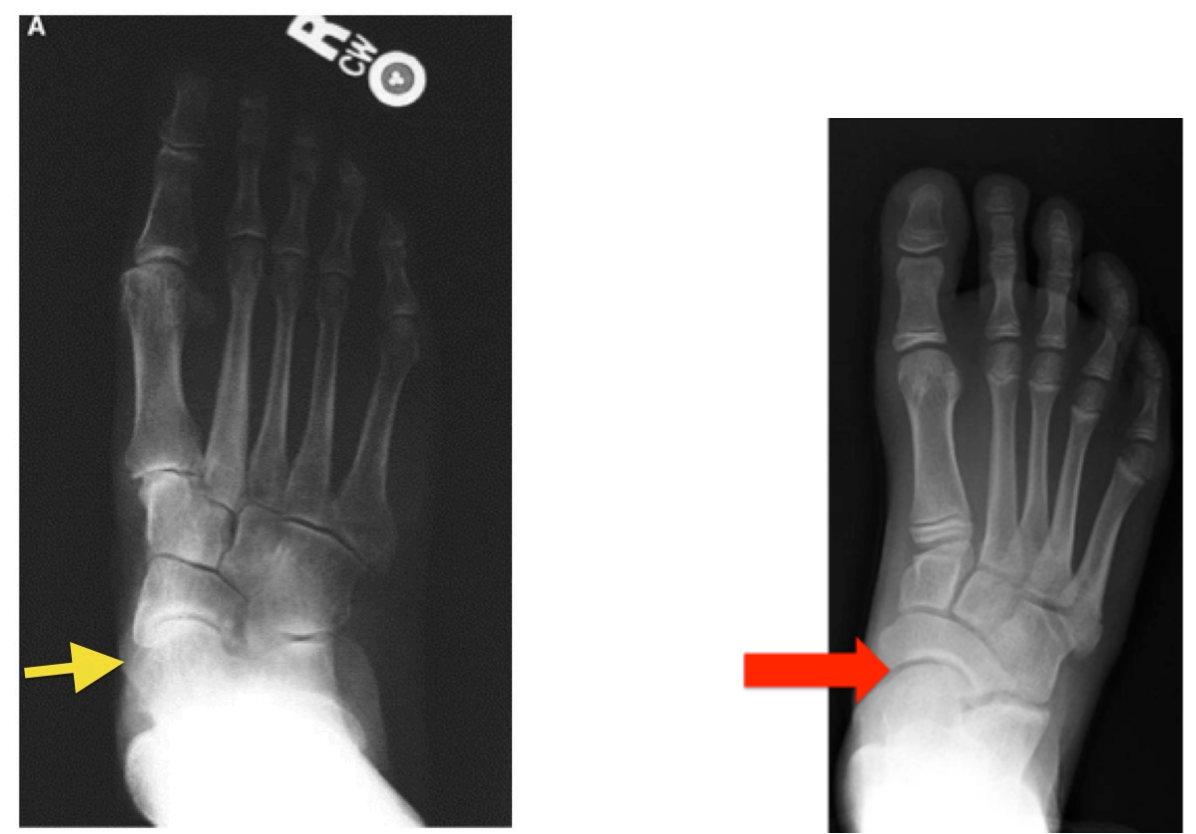

Figure 7: AP Radiograph: With talonavicular uncoverage as shown by yellow arrow (Van Boerum \& Sangeorzan, 2003) and normal encapsulated talonavicular joint shown by red arrow (Joseph Jr., 2008)

Clinically, it is important to diagnose during the early stages of AAFD because it is considered to be a progressive disorder (Durrant, Chokalingham, \& Hashmi, 2011) (Deland J. T., 2008) (Society, 2010). By diagnosing early, the possibility of saving the flexibility of the foot increases as the severity of the dysfunction decreases. Surgical intervention is common, however if early diagnosis occurs and conservative treatment measures are adopted it is possible to improve the possible pain level and continue normal living. If a streamlined measurement system with quantitative measures were adopted it may be easier for clinicians to diagnose earlier (Durrant, Chokalingham, \& Hashmi, 2011).

\section{MEDIOLATERAL MEASUREMENTS}

An important measurement in the development of flatfoot is calcaneal pitch (CP). This can be determined radiographically by drawing a line from the ground to the center 
of the calcaneus (Chen, Huang, Chen, Weng, Lee, \& Wang, 2006) (Blackman A., Blevins, Sangeorzan, \& Ledoux, 2009) (Greisberg, Assal, Hansen, \& Sangeorzan, 2005) and measuring the difference of the angle. The smaller the calcaneal pitch angle is, the closer to the ground the arch is, therefore the foot is more flat. Because calcaneal pitch varies by ethnicity (Castro-Aragon, 2009), each patient may have a different angle that is considered normal based on his or her specific characteristics.

Lateral talometatarsal angle (L-T1MT) is also a component in assessing the difference in angle between the first metatarsal and the talus. This measurement is taken by taking the midpoint of the talus between the margins of the talar neck through the posterior portion of the talus, where the first metatarsal axis is defined by two points in the proximal and distal metadiaphyseal junctions (Greisberg, Assal, Hansen, \& Sangeorzan, 2005) (Chen, Huang, Chen, Weng, Lee, \& Wang, 2006) (Blackman A. , Blevins, Sangeorzan, \& Ledoux, 2009) (Ellis, Yu, Johnson, Elliott, O'Malley, \& Deland, 2010) (Arangio \& Salathe, 2009). This angle should range within zero to four degrees in a normal foot (Chi, Toolan, Sangeorzan, \& Hansen, 1999) however in flatfoot the angle will be increased showing a deformity in arch height (Greisberg, Assal, Hansen, \& Sangeorzan, 2005).

The lateral talocalcaneal angle (TC) is an angle between the talar axis as described above as well as the midpoint of the longest point of the distal calcaneus to the closest point of the joint interface (Coughlin \& Kaz, 2009). As the flatfoot decreases this angle would decrease, as the bones will become more parallel when the foot is unstable. 


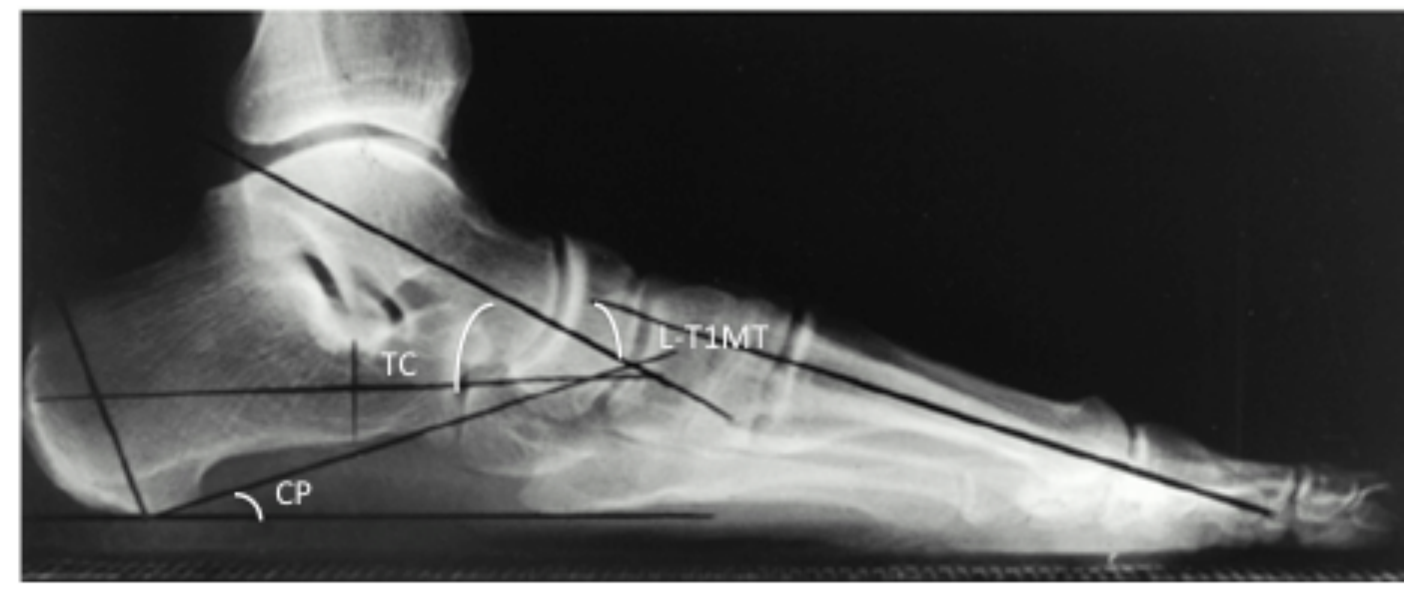

Figure 9: ML Angles: Showing TC, CP and T1MT and bony landmarks to create axis (Toolan, Sangeorzan, \& Hansen, 1999)

\section{ANTEROPOSTERIOR MEASUREMENTS}

Abduction normally occurs through the talonavicular joint uncovering the head of the talus and can be measured radiographically through comparison of the talus to the navicular angle (TN) (Van Boerum \& Sangeorzan, 2003) (Greisberg, Assal, Hansen, \& Sangeorzan, 2005) (Coughlin \& Kaz, 2009) (Murley, Menz, \& Landorf, 2009). The more the angle is uncovered, the flatter the foot.

A talar-first metatarsal angle (T-1MT) is considered where someone diagnosed with AAFD would have a larger angle than a normal foot (Blackman A. , Blevins, Sangeorzan, \& Ledoux, 2009). Measured as the center point of the navicular notches the talus is compared to the center point of the line from the first metatarsal ray (Chen, Huang, Chen, Weng, Lee, \& Wang, 2006) (Blackman A., Blevins, Sangeorzan, \& Ledoux, 2009). 


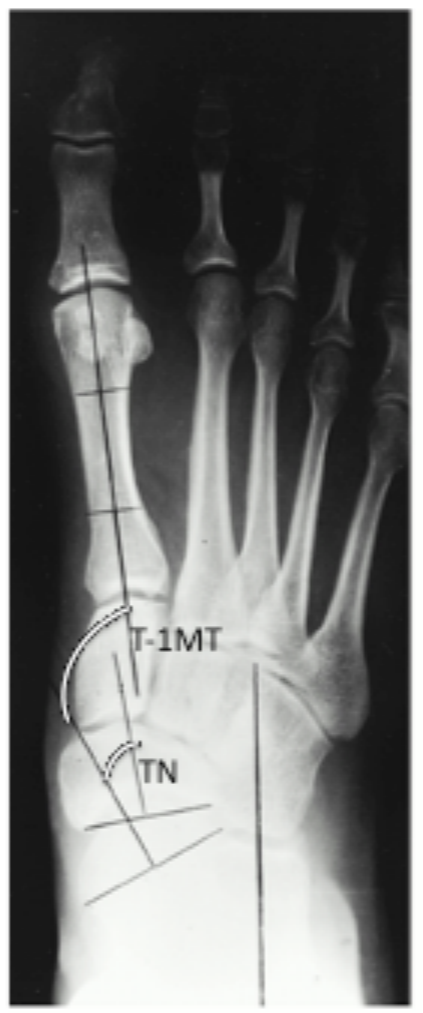

Figure 8: AP Measurements: Showing the angular measures of talonavicular and first talar metatarsal angle using the landmarks associated (Toolan, Sangeorzan, \& Hansen, 1999)

\section{PLANTAR PRESSURE MEASUREMENTS}

Plantar pressure measurements are indicative of distribution of load through the sole of the foot and can be taken while standing or while walking. This distribution is often separated into regions to focus attention on specific regions most affected by a particular disorder. By splitting the measurement into regions, the success of surgery can be compared against normal foot pressure data.

An easy measurement that can be taken from plantar pressure data to assess flatfoot is the arch index. If a patient has flatfoot, the midfoot area would be a larger percentage 
of a whole foot when compared to a normal foot as shown in Figure 10. By dividing the foot into thirds (forefoot, midfoot, hindfoot, excluding the toes) the midfoot area percentage is determined mathematically by dividing the area of the midfoot by the total area of the foot. (Chen, Huang, Chen, Weng, Lee, \& Wang, 2006) (Murley, Menz, \& Landorf, 2009) (Owen \& Burke, 2011) (Urry \& Wearing, 2001) (Cavanaugh \& Morag, 1999) (Yalcin, Esen, Kanatli, \& Yetkin, 2010). This measure is used to describe changes in area of the foot in both stance and walking that occur over time.
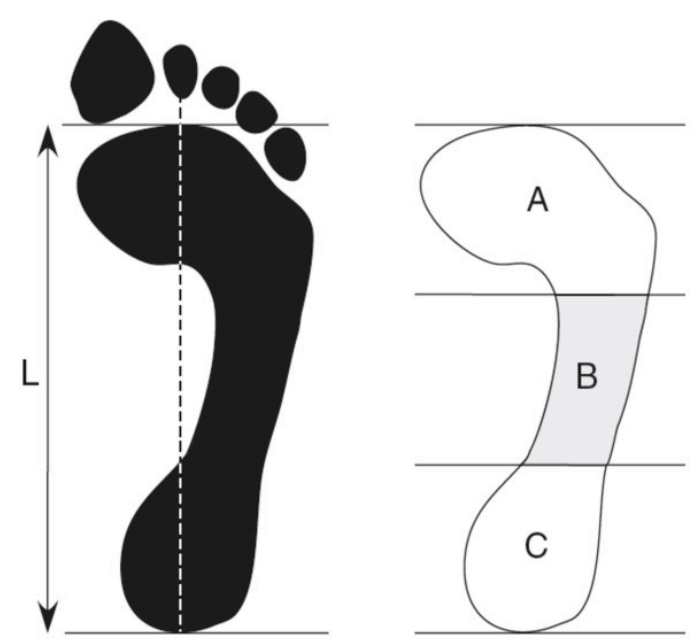

Figure 10: Arch Index measurement: Calculated by taking the area of $B /$ the area $(\mathrm{A}+\mathrm{B}+\mathrm{C})$ (Murley, Menz, \& Landorf, 2009)

The plantar pressure profile can be divided into distinct regions to monitor parameters such as peak pressure, force, and percentage bodyweight (Ellis, Yu, Johnson, Elliott, O'Malley, \& Deland, 2010) (Ledoux \& Howard, 2002) (Imhauser, Siegler, Abidi, \& Frankel, 2004) (Imhauser, Abidi, Frankel, Gavin, \& Siegler, 2002) (Yalcin, Esen, Kanatli, \& Yetkin, 2010) (Cavanaugh \& Morag, 1999) (Zammit, Menz, \& Muteanu, Reliability of the TekScan MatScan system for the measurement of plantar forces and pressures during barefoot level walking in healthy adults, 2010) (Menz \& Morris, 2006). These regions range from as simple as forefoot, midfoot and hindfoot to as complex as 
each region having a medial and lateral aspect, with individual metatarsals, greater toe, and lesser toes measured during both dynamic walking and stance. By creating regions, specific parts of the foot can be targeted and analyzed as seen in Figure 11. For the purpose of studying flatfoot deformity, the foot needs to be divided into medial and lateral regions, as it will be expected to see a medial shift in the midfoot when the arch collapses. Also, determining the effect of the metatarsal heads and toes are important to understand the mechanics of the soft tissue that is still intact but dysfunctional (Zammit, Menz, \& Muteanu, Reliability of the TekScan MatScan system for the measurement of plantar forces and pressures during barefoot level walking in healthy adults, 2010) (Ellis, Yu, Johnson, Elliott, O'Malley, \& Deland, 2010).

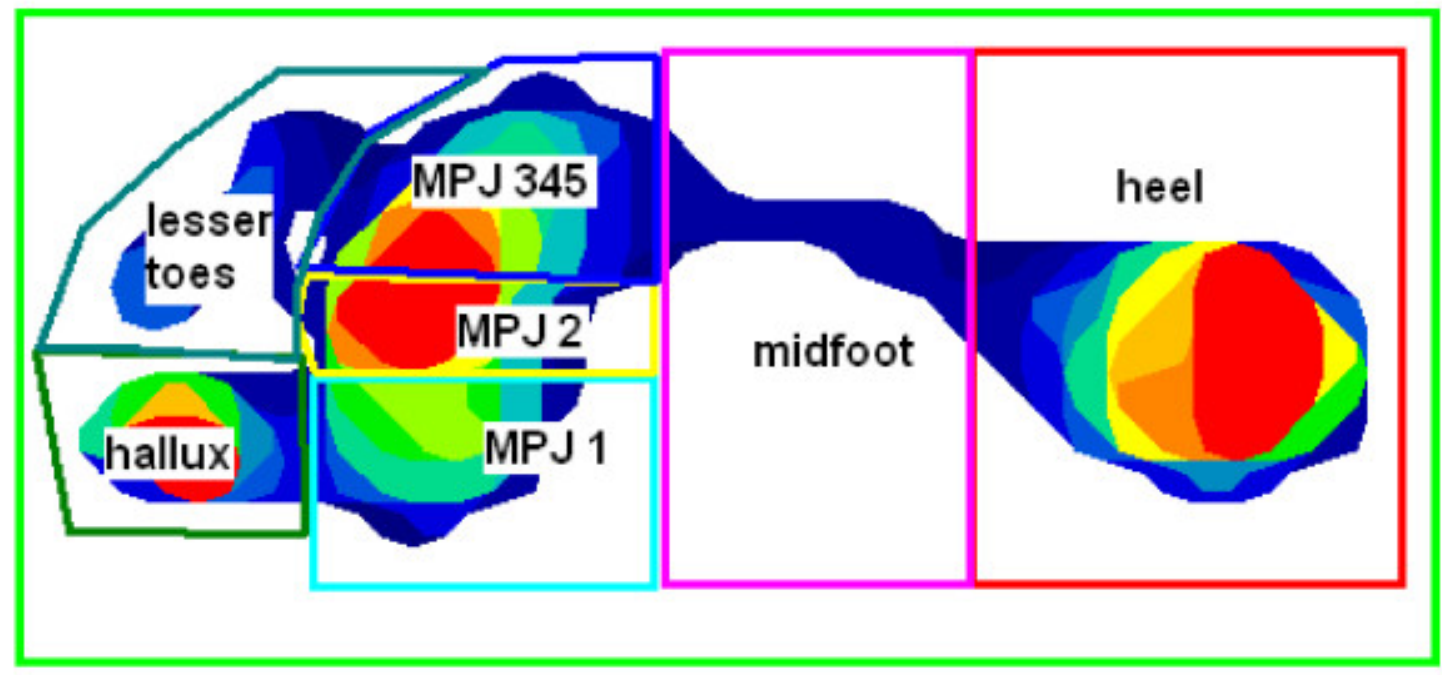

Figure 11: An example of the divisions of plantar pressure distribution where the foot was divided into 7 regions (Zammit, Menz, \& Muteanu, Reliability of the TekScan MatScan system for the measurement of plantar forces and pressures during barefoot level walking in healthy adults, 2010)

In an AAFD positive foot, plantar pressure distributions will increase on the first metatarsal head due to a lesser influence on the toes and increase in medial pressure due 
to flattening (Imhauser, Siegler, Abidi, \& Frankel, 2004) (Ledoux \& Howard, 2002).

Although difficult to quantify surgical success through the differences in plantar pressures, once corrected there should be a lateral shift in pressures through the midfoot and metatarsal regions (Ledoux \& Howard, 2002) (Imhauser, Siegler, Abidi, \& Frankel, 2004) (Ellis, Yu, Johnson, Elliott, O'Malley, \& Deland, 2010)

\section{QUANTIFYING SURGICAL RESULTS}

It is difficult to quantify the results of a "successful" flatfoot surgery because there are many contributing factors to the dysfunction as discussed earlier. There is no universally accepted procedure to follow in correcting flatfoot, however a few measures are obtained to compare a pre-operative state to a post-operative state. These include quantitative and qualitative measures based on radiographic and plantar pressure analysis as well as patient perception.

\section{SURVEYS}

A number of surveys are used to quantify how the patient feels before surgery and after to add to the quantitative data. Questions that are asked are of specific nature in determining if the surgery was successful for that particular patient. A helpful questionnaire that determines overall health and quality of daily life is the short- form survey where the patient fills out a general questionnaire of 36 questions (SF-36). This form is used in general health surveys. It can be used as a starting point for understanding the patient's mental and physical weaknesses.

There are other surveys that have been used to score foot and ankle function. A 
newer foot and ankle outcome score is the Visual Analogue-Scale of the Foot and Ankle (VAS-FA) where Richter et al asked twenty subjective questions and correlated the results with the SF-36 form (Richter, 2006). This scale mainly focused on function of the foot and ankle rather than the pain. One of the pitfalls to this scaling is that it has not been used in many scholarly articles; therefore, the validity of the scaling cannot be determined. Other scales such as the Foot and Ankle Ability Measure (FAAM) and Foot and Ankle Disability Index (FADI) mainly rely upon activities of daily living and sports related injuries (Masaheri, 2010) and should not be applied to acquired flatfoot. Another lesser-known scale, the foot function index (FFI) is a scale that has 23 items divided into three subscales. All the questions are subjective in nature, but proved to be a moderate level of correlation between the AOFAS and the FFI (Ibrahim, Beiri, Azzabi, Best, Taylor, \& Menon, 2007). Both surveys provided quality of life info with acceptable reliability (Ibrahim, Beiri, Azzabi, Best, Taylor, \& Menon, 2007).

Throughout the literature, the AOFAS survey seemed to be popular but recent limitations to the study reflect inconsistencies in data analysis. According to Guyton et al, the AOFAS scores show limited precision due to small number of response intervals that skewed analysis when a small change in an answer had dramatic effects on the results (Guyton, 2001). The AOFAS survey is a bit different than the other surveys that give general information about the foot and ankle. Instead this survey is broken into part of the foot including the hindfoot, midfoot, and forefoot which is then further subdivided into hallux versus metatarsal phalangeal joints (Guyton, 2001). This may have a limitation to the patient as they would have to take multiple surveys if more than one part of the foot has symptoms. Although this survey has been used in multiple facets of literature, 
recently it has been in question as to the validity of the scoring and is strongly advised not to be used according to AOFAS website.

The survey that is recommended for research with the demise of the AOFAS system, is the Functions Ankle Outcome Score (FAOS) which incorporates patient's pain levels, symptoms, activities of daily living, quality of life and recreation (Negahban, 2010). With a strong correlation to the SF-36 the validity of the FAOS questionnaire is a positive source for reliability ratings and administration time (Negahban, 2010). This survey is 42 questions and is a subjective test for the patient to report how they feel at the time of before surgery and after.

Questionnaires allow for researchers to quantify qualitative results and compare how the patient feels both before and after surgical intervention. Quality of life and resuming activities of daily living are important assessment tools in determining the success of the surgery.

\section{OBJECTIVE}

The purpose of this study was to determine the quantitative biomechanical and qualitative survey differences between pre-operative and post-operative states of patients undergoing surgical correction for Stage IIb Adult Acquired Flatfoot Deformity. Forces

and pressures acting on the foot during stance and walking were measured. Surveys were implemented to assess patient's perspective of quality of life before and after surgical intervention. 


\section{METHODS}

\section{PARTICIPANTS}

Overview: an Institutional Review Board protocol was submitted to test participants that were diagnosed with Stage IIb Adult Acquired Flatfoot Deformity. Surveys were distributed consisting of the SF-36 general health form as well as the FAOS to determine how each participant viewed their injury. Tests were performed both pre-operatively and post-operatively including radiographs, MRI, plantar pressure and gait-analysis.

Radiographs were taken in a weight-bearing one-foot stance from a mediolateral and anteroposterior view. An MRI scan was done only pre-operatively for another related study. Plantar pressure protocol was performed for both walking and stance states. Gait analysis using an infrared camera system was performed as well with walking trials for another related study. The Institutional Review Board granted ethical consent for the study (HM13044) where participants were informed of procedure and all participants before data collection signed a consent form.

Participants were recruited for evaluation from the Foot and Ankle Clinic at 
Virginia Commonwealth University. Participants that were diagnosed with Stage IIb flatfoot requiring surgical correction by a physician were considered for the study. Although no gender discrimination in participants occurred, the entire population consisted of females between ages 26 to 69 . Recruitment materials provided were a poster explaining what tests were going to be executed, what was expected of the participant, and another document explaining the legal rights of each participant. With a seal of IRB approval, the document explaining the benefits of participating in the study was kept by each participant and a consent form was signed. A contact form was also filled out by the participant, which included information pertaining to age, race and gender. The documents and researchers informed participants that at any time they felt uncomfortable and wanted to withdraw from the study they could do so without any implication. A one-year follow up visit was required post-operatively where the same testing would be performed.

\section{SURVEY INSTRUMENTS}

A general health form, SF-36 (Appendix), was given to the participant to fill out both pre-operatively and post-operatively to qualitatively measure well-being. The SF-36 asks 36 questions pertaining to physical functioning, role limitations due to physical health, role limitations due to emotional problems, energy/fatigue, emotional well-being, social functioning, pain and general health. Each question was randomized and scored based on percentage for each particular question with a total score percentage as the average of all the questions.

Specifically for the foot and ankle, a Foot and Ankle Outcome Score (FAOS) 
(Appendix) survey was given to participants as well to measure the effect of their injury on daily life. The FAOS survey was 42 questions pertaining to the involvement of the foot and ankle with symptoms, pain, activities of daily living, sports and recreation, and quality of life. Each question was set up in the five sections listed, and was assigned a certain number of points per question. The total FAOS score is out of 500 points where 500 points would be one-hundred percent functioning.

Although there are many other surveys that have been used, a correlation with the FAOS questionnaire and SF-36 has been made to assess function of the foot and ankle in a similar pre-operative and post-operative study (Ellis, Yu, Johnson, Elliott, O'Malley, \& Deland, 2010). There was debate over using the AOFAS (American Orthopedic Foot and Ankle Society) scoring instead of FAOS scoring, however, the AOFAS scoring was tested and showed limited precision due to small number of small response intervals (Guyton, 2001). If there was a small change in response with the AOFAS survey, a drastic difference in results was seen (Guyton, 2001). Also, the AOFAS scoring system broke down the foot into regions including, hindfoot, midfoot, forefoot, and hallux with metatarsolphalangeal joints where each region required a different survey. Since the researchers in this particular study were looking at the entire foot as a whole, it was more appropriate to use the FAOS survey structure to assess functional outcome.

\section{MEASUREMENT APPARATUS}

Radiographs were obtained on the third floor of VCU department of Radiology. A standard radiograph machine was used and operated by the same personnel each time for each participant. Weight-bearing two-foot stance was taken in the anteroposterior view, 
which was then analyzed in this particular project. A piece of currency was placed on the film to quantify any magnification that may have occurred. A mediolateral view was also taken where the participant stood on a box, with a handle for balance, and was asked to raise the non-afflicted leg for a single weight-bearing stance capture. The mediolateral view was used in a different project.

Plantar pressures and forces were obtained using the TekScan MatScan ${ }^{\circledR}$ system Model 7101 E (Boston, MA, USA). The pressure mat system contains 8448 resistive sensors $\left(3.9\right.$ sensors $\left./ \mathrm{cm}^{2}\right)$ in a $5 \mathrm{~mm}$ thick floor mat $(542 \times 502 \mathrm{~mm})$ where the sampling frequency averaged $60 \mathrm{Hertz}(\mathrm{Hz})$ (Figure 12). The plantar pressure mat was affixed to the floor using masking tape in the four corners to prevent any movement. The handles were attached and cords were taped to the floor, to prevent a possible tripping hazard. A "runway" of about 5 meters was then taped on the floor to direct participants to the plantar pressure mat. Using the TekScan 6.40 software that interfaces with the plantar pressure mat, the foot pressure was analyzed and categorized (Figure 13). 
Each participant was weighed in order to calibrate the plantar pressure mat. Calibration can be achieved by different techniques integral with the software. The calibration techniques available include a point, step, walk and frame calibration. For point calibration the participant would need to calibrate both left and right feet separately, and while in video mode, the calibrations show low forces due to the linear relationship between raw sensels fired to force on the mat (Tekscan, Incorporated, 2008). A step calibration process is more beneficial to the study because the participant is asked to stand and walk on the mat which step calibration allows for the computer to analyze the force from the foot and the sensels that are fired (Tekscan, Incorporated, 2008). Step calibration has the participant step onto the mat with one foot (a wall may be used to steady themselves) where the mat then calibrates the force of one foot and bodyweight to determine the threshold for each participant for the rest of the testing procedure. Step calibration reduces trial-to-trial variation when compared to point calibration because it has a factor for dynamic changes as well as time related changes (Tekscan, Incorporated, 2008). Walk calibration was not as accurate as step calibration, but this was mainly for participants that cannot stand on one leg for ten seconds. Walk calibration has many stipulations for a successful calibration including the "toe off" pressure to be similar to heel strike, which would not hold true with AAFD participants.

\section{PROCEDURE}

Stance measurements were obtained using a two-foot stance phase and a one-foot stance phase. Stance measurements mimic previous cadaveric studies where flatfoot was 
created surgically (Scott, Hendry, Iaquinto, Owen, Wayne, \& Adelaar, 2007). Although a fixed force (lbs) was used on cadaveric specimens, the change in the distribution patterns from normal to flatfoot was apparent (Scott, Hendry, Iaquinto, Owen, Wayne, \& Adelaar, 2007). Stance data was acquired by allowing the barefoot participant to step on the mat for twenty seconds. A delay of ten seconds was added so that the participant was able adjust their balance. Ten seconds were recorded with the participant remaining as still as possible. For one-foot stance, the participant was offered a chair to hold onto in case s/he could not balance because of discomfort, with the afflicted foot (Figure 14). The participant could also choose not to stand on the afflicted foot if there was too much pain. One trial of each foot and two-foot stance was obtained in the pre-operative and post-operative states.

A walking pressure profile was collected as well where a three-meter walkway was provided between the participant and the plantar pressure mat. The participant walked at a self-selected speed via the walkway over the plantar pressure mat while looking ahead. This was done to ensure that there were no gait changes when the participant felt the mat under their bare foot. To make certain that the participant did not look at the mat, an object was placed at eye level at the end of the runway that they were instructed to look at the entire time (Figure 14). Three trials were practiced to make sure that the desired foot hit the mat. Once the three practice trials were performed the subject was recorded via triggering capabilities of the TekScan ${ }^{\circledR}$ software. In the literature, three trials have been sufficient in providing reliable force and pressure data when trials were executed correctly (Zammit, Menz, \& Muteanu, Reliability of the TekScan MatScan system for the measurement of plantar forces and pressures during barefoot level walking in healthy 
adults, 2010) (Menz, Munteanu, Zammit, \& Landorf, 2010) (Menz, Fotoohabadi, Munteanu, Zammit, \& Gilheany, 2012) (McPoll, Dupuis, \& M, 1999).

Trials were discarded and repeated if there was a disturbance in gait such as a heel scuff across the mat or abnormal movement. If the participant paused while walking on the mat, did not look at the object eye level, or did not continue to walk past the mat, the trial was also rejected and repeated (Figure 13). If the plantar pressure recording did not encompass the whole foot on the mat, this trial was then discarded as well. The number of successful trials always totaled three for each foot, afflicted and normal. The actual number of trials performed, however, was participant specific.

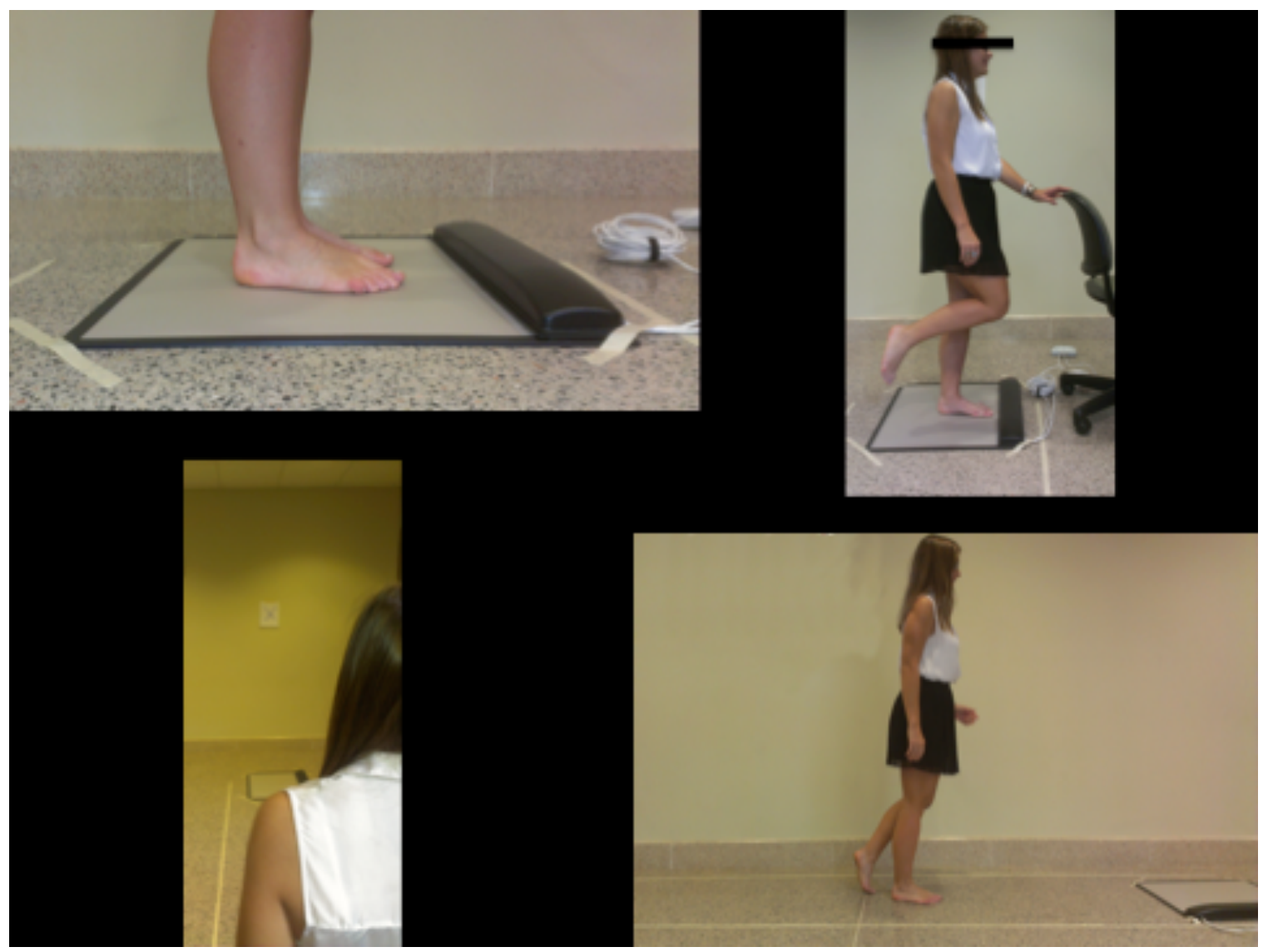

Figure 14: From left to right, top to bottom: Two-foot stance on plantar pressure mat. One foot stance with use of a chair to balance if needed. Walkway with image at eye level to keep eyes in correct position. Walking trial with plantar pressure mat. 
After meeting with the physician and health status cleared, one-year post-operative follow up was completed on participants where the procedures were repeated as stated above. One year was chosen to be certain that the participant would have ample time to recover from surgery and ascertain a new steady walking cycle (Deland J. T., 2008). Repeatability was ensured through the three trial method, but each participant will have variability.

\section{DATA ANALYSIS}

Maximum force (through percent body weight) and peak pressure were the main parameters determined from the plantar pressure profiles in nine regions of the foot (Figure 15). A measure of percent body weight was chosen to quantify results over time with a potential shift in weight after surgery. This measurement was taken by using the maximum force (lbs) output of the MatScan ${ }^{\circledR}$ system and dividing by the participant's bodyweight. For two-foot stance, the divisor was obtained by finding the force distribution of both feet because the participant may favor one foot over the other. Peak pressure measurements were acquired through the standard output of the MatScan ${ }^{\circledR}$ system and collected. Both maximum force and peak pressure measurements have been found to be important in determining the efficacy of treatment of developmental foot problems (Menz, Munteanu, Zammit, \& Landorf, 2010) (Menz, Fotoohabadi, Munteanu, Zammit, \& Gilheany, 2012) 




Figure 15: Nine region discrimination with greater toes, lesser toes, $1^{\text {st }}$ metatarsal head, $2^{\text {nd }}$ metatarsal head, 3-5 metarsal heads, medial and lateral midfoot, and medial and lateral hindfoot.

Regions were selected based on previous studies to provide insight into the independent function of each respective segment of the foot (Scott, Hendry, Iaquinto, Owen, Wayne, \& Adelaar, 2007) (Zammit, Menz, \& Muteanu, Reliability of the TekScan MatScan system for the measurement of plantar forces and pressures during barefoot level walking in healthy adults, 2010) (Ellis, Yu, Johnson, Elliott, O'Malley, \& Deland, 2010) (Menz, Fotoohabadi, Munteanu, Zammit, \& Gilheany, 2012). Using the measurement tool in the MatScan ${ }^{\circledR}$ software, the foot was first divided into thirds, from the midpoint of the heel of the foot to the second toe, yielding the forefoot, midfoot and hindfoot. To distinguish between the medial and lateral regions of the midfoot and hindfoot a measurement line was made from the second toe to the middle of the heel of the foot as described by the TekScan ${ }^{\circledR}$ template of 13 regions and 3 segment lines. A perpendicular line was then drawn for each distinct region of the forefoot, midfoot, and hindfoot (Figure 16). 


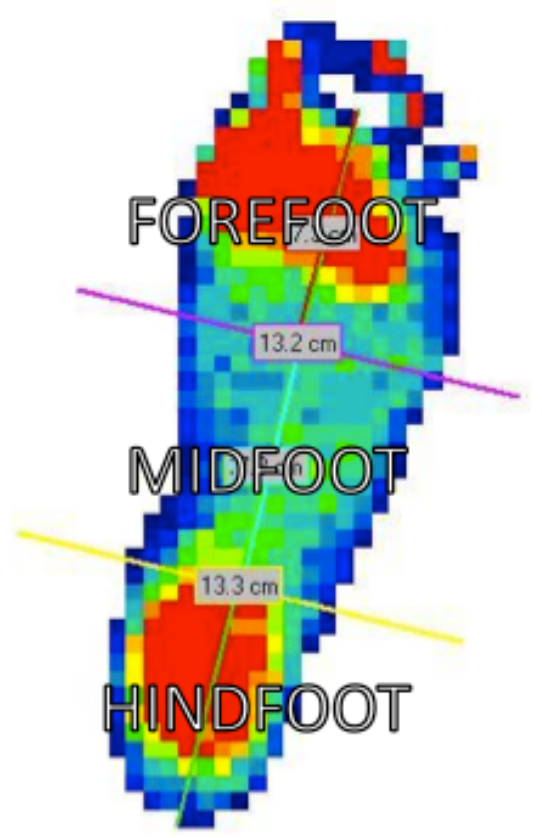

Figure 16: Walking Trial divided into three distinct regions

If the measurement did not equally divide into three, preference was given to the forefoot, midfoot then hindfoot for potential "extra" pressure sensing cells as has been done previously (Scott, Hendry, Iaquinto, Owen, Wayne, \& Adelaar, 2007). To distinguish the metatarsals in the forefoot, the metatarsal head locations were determined by overlaying the AP view radiograph on the two stance trials and one walking trial using Adobe Photoshop ${ }^{\circledR}$ (Figure 17). The framework from the overlaid walking trial was then used on following trials, mildly adjusting some areas to fit the foot due to magnification in radiograph. 


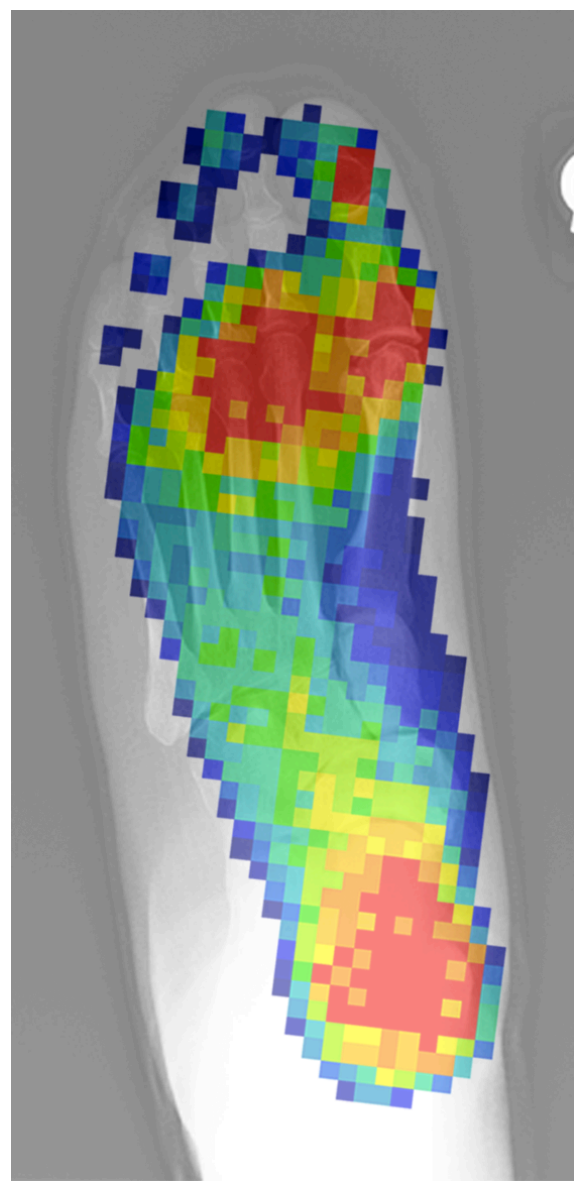

Figure 17: AP radiograph overlay of a walking trial showing distinct metatarsal heads.

Once the nine regions were determined (Figure 15), peak pressure and percent body weight in each region were calculated. Pre-operative and post-operative regions were compared over all participants using a paired t-test for each respective region to determine if the differences were significant. Differences were also compared within a participant for a pre-operative and post-operative state. Both one-foot and two-foot stances were analyzed individually for the afflicted foot with one trial each for preoperative and post-operative states. For the three walking trials, each trial was averaged based on regional profile and then compared before and after surgical intervention.

Arch index was also measured using the forefoot, midfoot, and hindfoot 
distinctions from the previous framework (Figure 16) without the medial and lateral divisions. Arch index was measured by taking the maximum area for each trial and the formula below was used to calculate the arch index (Zammit, Menz, \& Muteanu, Reliability of the TekScan MatScan system for the measurement of plantar forces and pressures during barefoot level walking in healthy adults, 2010):

$\frac{\text { Midfoot }}{\text { Forefoot }+ \text { Midfoot }+ \text { Hindfoot }}$

According to the calculation, the arch index quantifies the percentage of the midfoot that is touching the plantar pressure mat. For stance trial afflicted foot was analyzed for onefoot and two-foot stance. For walking trials, the average of the three trials was taken at the maximum area.

When the three trials were assessed, a bimodal graph was seen in the distribution of load over contact time with the floor within walking trials (Figure 18). This is typically used to determine each participant's personal differences in walking, but in this project it was used to determine the frame used for calibration of each participant. 


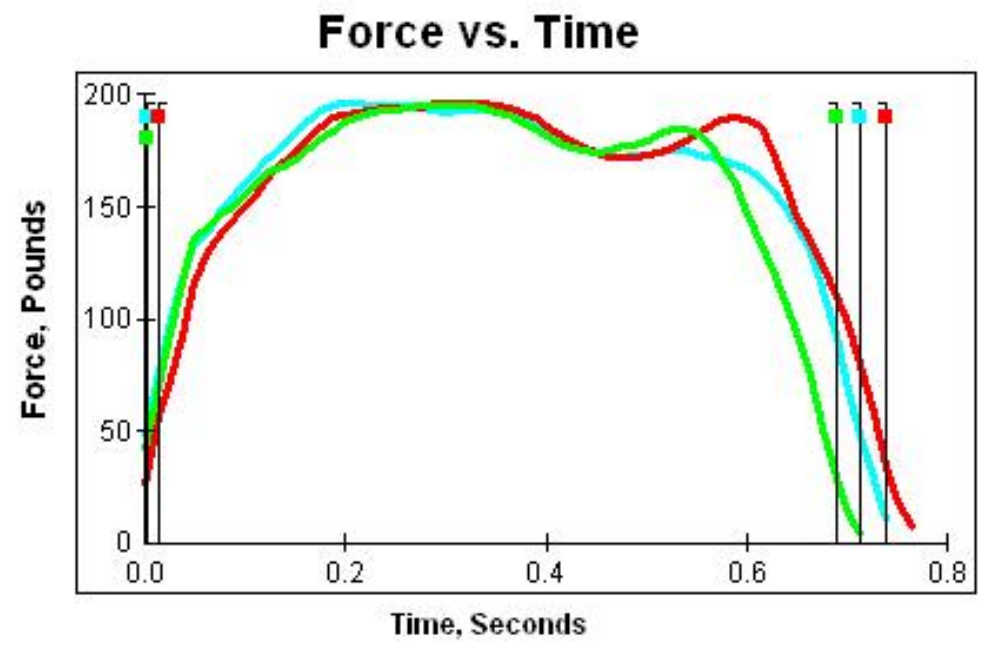

Figure 18: A bimodal walking trial graph illustrating three trials of one participant (green, blue, red) that was used for frame calibration.

Three trials for each participant are shown in Figure 18, with the different colored lines indicating each trial. As shown, each trial varies within a participant but an overall general curve for each participant can be seen. The general curve of gait is shown where the first peak was the heel strike and the second peak was toe-off. Midstance can be found between the two peaks, usually where the graph dips in force.

The frame used for walking calibration was determined with the use of the bimodal graphs, where the midstance of the gait cycle was used as the calibration point for total force. Using each participant's weight measurements, the trials were calibrated after they were recorded. Stance frame calibration was determined by the most stable trial that occurred over the largest frame span where the middle frame was used over the stable trial. 


\section{RESULTS}

There were a total of six participants in this study that were available for the preoperative testing including radiographs, an MRI, plantar pressure testing and gait analysis using a VICON ${ }^{\circledR}$ system. The radiographs, MRI, and gait analysis were used for a different component of this study. After one-year in which the attending physician cleared each participant as recovered from their surgery, post-operative evaluations were performed. Post-operative testing had a total of five returning participants, where the second participant did not return because she could not be contacted.

Each participant was analyzed individually to determine the effectiveness of his or her particular surgical results based on plantar pressure measurements. Collective results of pre-operative and post-operative plantar pressures were compared including the preoperative results of participant two who did not return for a one-year follow-up. Participants were labeled anonymously based on time of entrance into the study where no names were used just a label of "P" then a number.

In the following sections, individual results are shown for two of the six total 
participants. These participants were chosen because they represent the least flat-footed and most flat-footed participants based on the walking arch index. Participant 1 was the least flat and subsequently Participant 5 was the most flat. With the comparison of plantar pressure images of each participant, Participant 1 shows no midfoot collapse preoperatively where there is a defined midfoot collapse pre-operatively for Participant 5. Because of the distinct characteristics of these particular participants, they reflect the population at both extremes of this study.

Group results for all six participants follow the two participants highlighted, where a total representation of surgical success is observed. It is to be noted that the other participant's individual profiles analyzed in the group results can be found in Appendix $\mathrm{B}$, because each participant had personal variance.

\section{PARTICIPANT 1 (Right foot afflicted)}

Participant 1was first brought in on 9/23/2010 for plantar pressure analysis, radiographs, and motion analysis. Plantar pressure protocol was done first at the Ambulatory Care Center and then radiographs were taken first at third floor Gateway of MCV then the participant traveled for motion analysis.

Participant 1 was brought back on 10/13/2011, 385 days after the initial visit, for plantar pressure analysis and radiographs. No motion analysis was performed. Plantar pressures were evaluated in the Ambulatory Care Center in a spare protocol room. Radiographs were taken at third floor Gateway of MCV.

\section{Pre-Op Comments:}


The participant came in with pain in her foot and complained of her hip hurting slightly. She came in the morning with a boot on her foot and it was a rainy day, which may have contributed to her extra pain perception. Her gait pattern was uninhibited despite injured foot. Two-foot stance was performed as well as one-foot stance with only a small usage of the chair in front of her

Three trials of each walking analysis of each foot were performed, and when compared to normal trials, the participant looked similar. Both stance trials also looked similar to a normal foot.

\section{Post-Op Comments:}

After surgical correction via a medial calcaneal osteotomy (MCO) and tendon transfer, the participant returned for testing. Upon arrival, the participant stated that she had hip pain again, on her right side, this time much more severe than the last time seen. Her gait was much more choppy clearly favoring her non-operative side (left foot). Her step spacing was much smaller than her pre-operative visit, which led to two feet capture during walking analysis. Even though this occurred, each foot was analyzed individually, but she did have to do many more trials than pre-op. She also spent a considerable amount of time resting between trials, something that she did not do before. Both twofoot and one-foot stance were captured and analyzed.

\section{Surveys:}

The SF-36 is a determinant of overall health and quality of life on a percent based scale where if the participant is closer to 100 then general health is good. 
SF-36

Pre-Op: 56

Post-Op: 79

Although there was an increase in post-operative scoring of the SF-36 form, general discomfort from her post-operative visit may have influenced her perception of her overall general health. Because the general health survey also includes emotion, participant 1 expressed loss over her job termination, which may also lead to a lower general health form score.

The FAOS (foot and ankle outcome score) is a determinant of the health of the foot and ankle as well as scaling the level of activities of daily living and overall health due to foot and ankle problems. This scale is out of 500, where the higher the total score, the less affected the foot and ankle issue is to the participant.

\section{FAOS}

Pre-Op: 286

Post-Op: 448

A dramatic increase in foot and ankle scoring seen post-operatively suggests that the surgical correction helped this participant. After surgery the participant is at $90 \%$ functioning of the foot and ankle versus the $57 \%$ pre-operative.

\section{Plantar pressure images:}

Participant 1's individual plantar pressure pictures are shown at peak pressure over time (Figures 19 and 20). These images show the maximum amount of firing on each sensel over total contact time on the plantar pressure mat. For the stance measurement, the largest group of frame captures was used where the participant moved 
very little. The middle frame of the group was chosen as the most steady and used for calibration. Walking images taken are over the total time it took for the participant to go from heel strike to toe off.

\section{PRE-OP PLANTAR PRESSURE IMAGES}

\section{$\underline{\text { PARTICIPANT } 1}$}

\section{Two -Foot Stance}

$\underline{\mathrm{kPa}}$
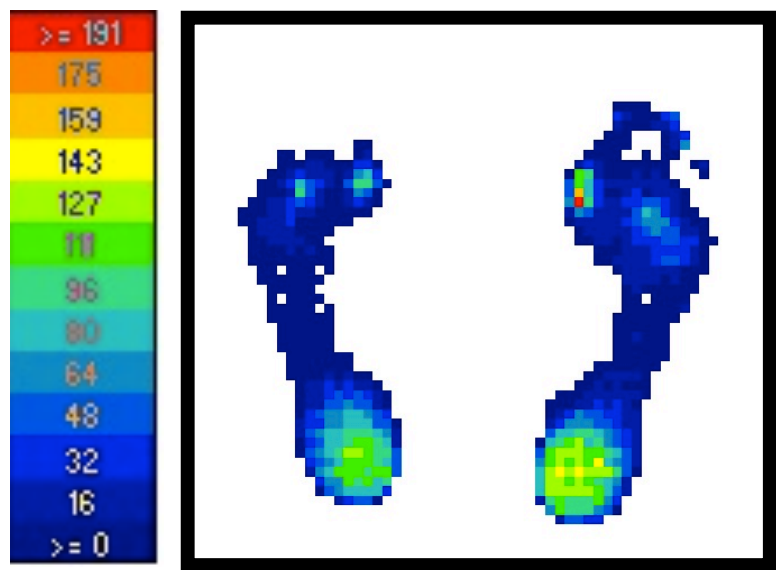

Walking

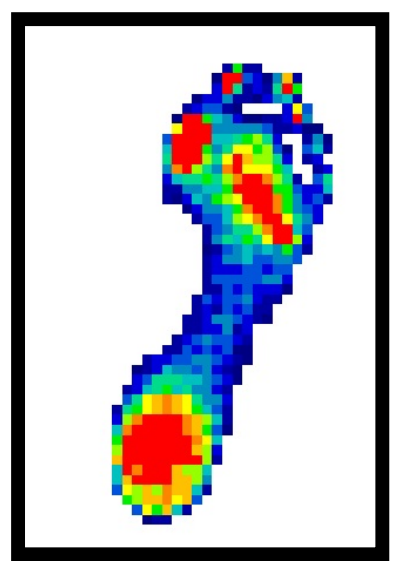

$\underline{\text { Trial } 1}$

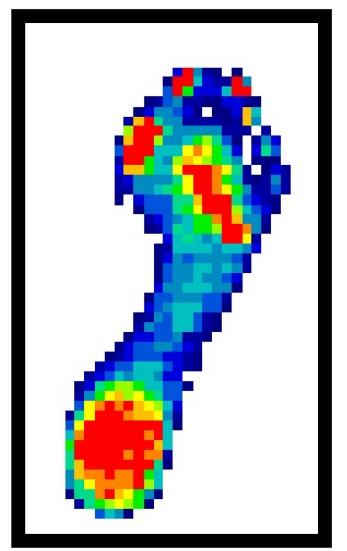

$\underline{\text { Trial } 2}$



$\underline{\text { Trial } 3}$

Figure 19: Pre-Op plantar pressure images for Participant 1 


\section{POST-OP PLANTAR PRESSURE IMAGES}

PARTICIPANT 1

$$
\text { Two-Foot Stance } \quad \text { One-Foot Stance }
$$

$\underline{\mathrm{kPa}}$
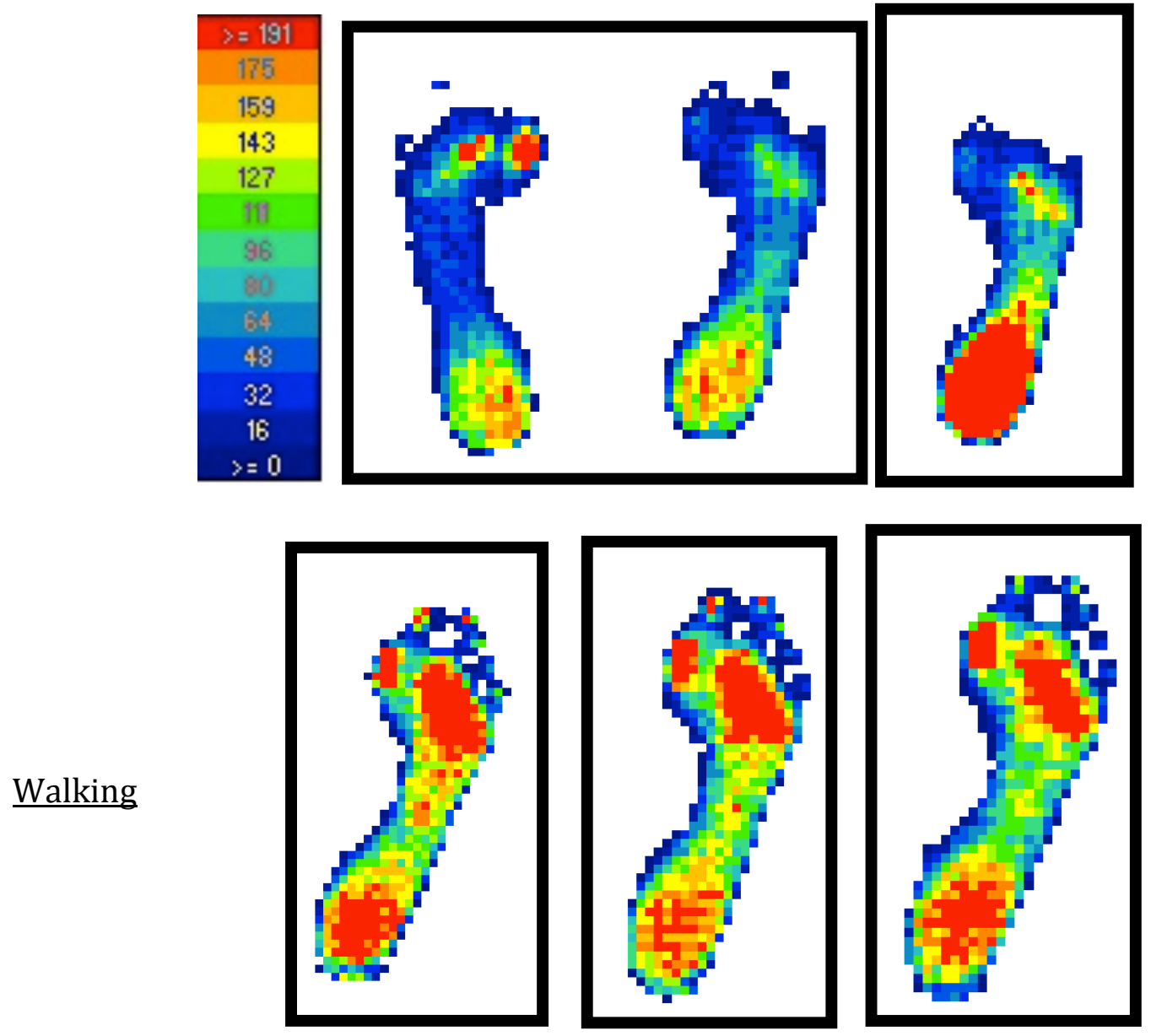

$\underline{\text { Trial } 1}$

Trial 2

$\underline{\text { Trial } 3}$

Figure 20: Post-Op plantar pressure images for Participant 1

\section{Walking:}

Three trials of each foot for walking analysis was performed and compared to preop analysis. Calibration of the plantar pressure mat was done by frame calibration where for each walking trial the middle frame of midstance was used. 


\section{Peak Pressure:}

Peak pressure is measured by taking the peak pressure in each region over the total time of the walking cycle. Each peak represents a distinct instant of time in the gait cycle for each region. This does not represent only one event in time, but nine separate events.

\section{WALKING PEAK PRESSURE}

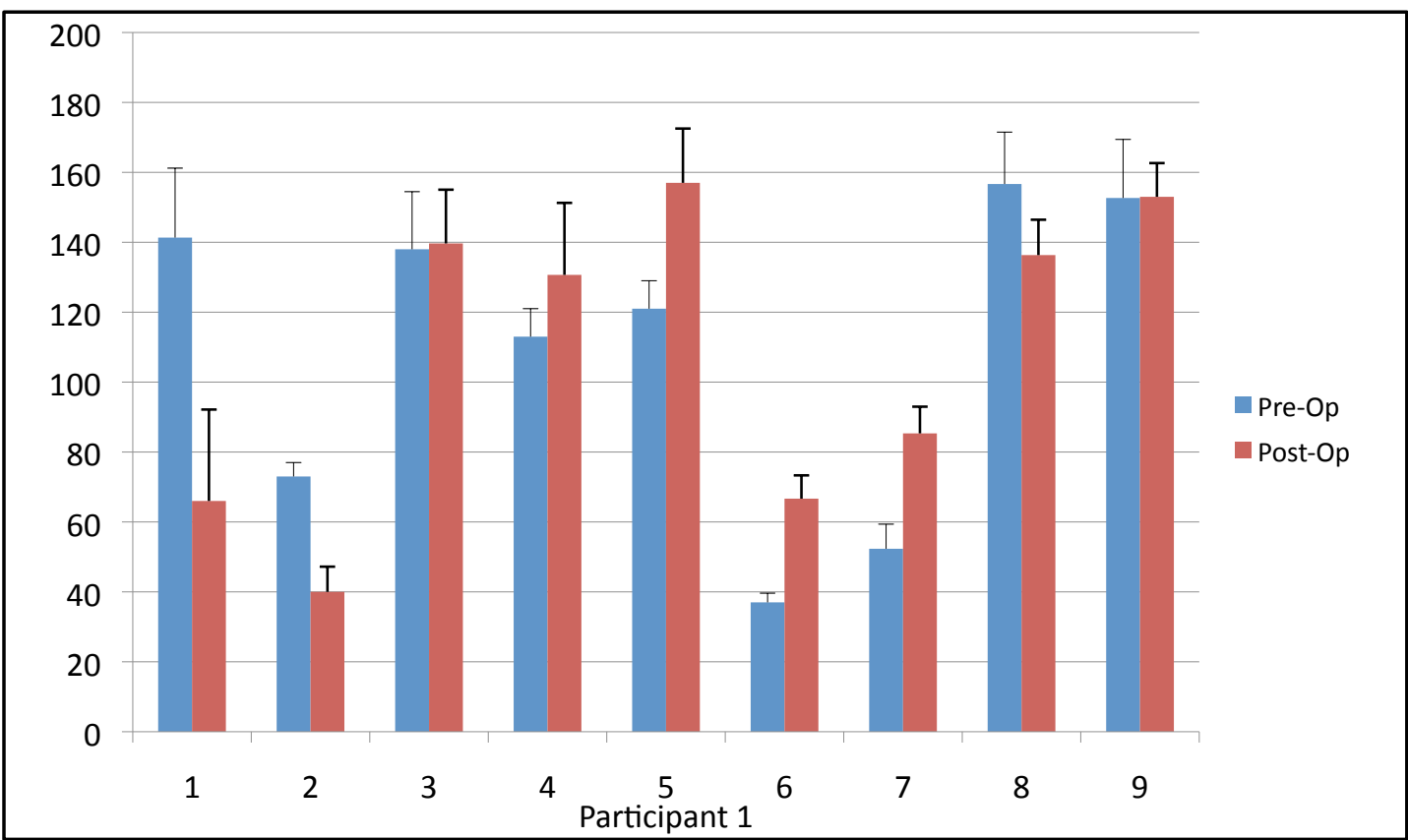

Figure 21: Peak pressure in each of the nine regions defined over the entire walking cycle averaged over three trials of the afflicted foot for Participant 1.

Pre-Op Max Pressures $(\mathrm{kPa})$ :

\begin{tabular}{|l|r|r|r|r|r|r|r|r|r|}
\hline Regions & 1 & 2 & 3 & 4 & 5 & 6 & 7 & 8 & 9 \\
\hline Average & 141 & 73 & 138 & 113 & 121 & 37 & 52 & 157 & 153 \\
\hline $\begin{array}{l}\text { Standard } \\
\text { Deviation }\end{array}$ & 20 & 4 & 16 & 8 & 8 & 3 & 7 & 15 & 17 \\
\hline
\end{tabular}

Post-Op Max Pressures (kPa)

\begin{tabular}{|l|r|r|r|r|r|r|r|r|r|}
\hline Regions & 1 & 2 & 3 & 4 & 5 & 6 & 7 & 8 & 9 \\
\hline Average & 66 & 40 & 140 & 131 & 157 & 67 & 85 & 136 & 153 \\
\hline $\begin{array}{l}\text { Standard } \\
\text { Deviation }\end{array}$ & 26 & 7 & 15 & 21 & 16 & 7 & 8 & 10 & 10 \\
\hline
\end{tabular}


Peak pressure measurements increased post-operatively in most regions, except the toes (regions 1 and 2) and region 8. It is particularly concerning that there is an increase in pressure in the medial midfoot (region 6) because with surgical correction it is expected to decrease with an increase laterally (region 7).

\section{Percent Body Weight:}

The measurement of percent body weight in each region was taken by the maximum force in pounds in that region on the afflicted foot and dividing it by the total body weight of the participant. This was done so that the measurements can be compared across time if the bodyweight of the participant changed. The participant was weighed both pre-operatively (147.61bs) and post-operatively (164.4 lbs).

WALKING PERCENT BODY WEIGHT

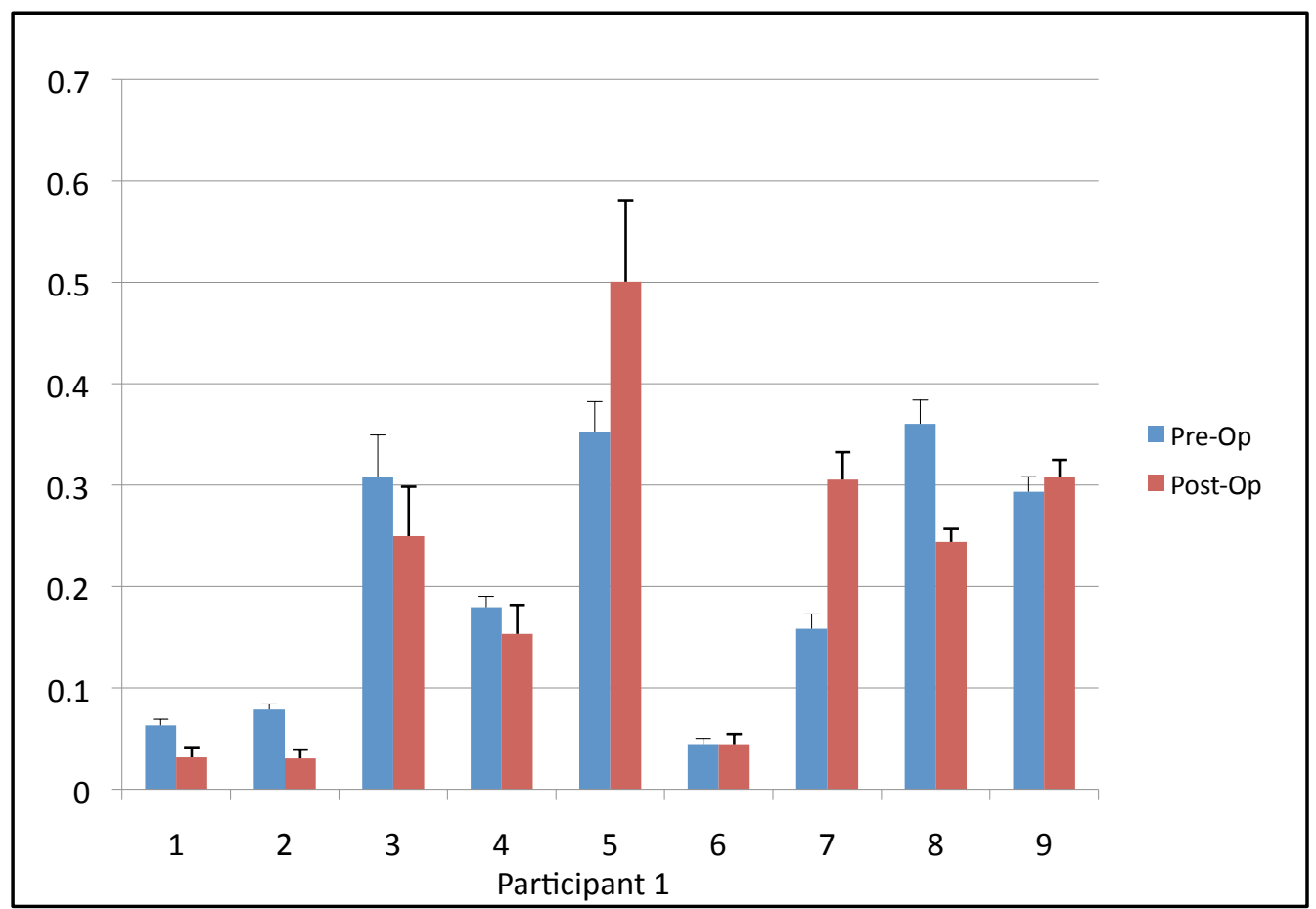

Figure 22: Maximum percent body weight in each of the nine regions over time of the walking trial for the average of three trials on the afflicted foot for Participant 1. 
Pre-Op Percent Body Weight

\begin{tabular}{|l|r|r|r|r|r|r|r|r|r|}
\hline Regions & 1 & 2 & 3 & 4 & 5 & 6 & 7 & 8 & 9 \\
\hline Average & 0.06 & 0.08 & 0.31 & 0.18 & 0.35 & 0.04 & 0.16 & 0.36 & 0.29 \\
\hline $\begin{array}{l}\text { Standard } \\
\text { Deviation }\end{array}$ & 0.01 & 0.01 & 0.04 & 0.01 & 0.03 & 0.01 & 0.01 & 0.02 & 0.01 \\
\hline
\end{tabular}

Post-Op Percent Body Weight

\begin{tabular}{|l|r|r|r|r|r|r|r|r|r|}
\hline Regions & 1 & 2 & 3 & 4 & 5 & 6 & 7 & 8 & 9 \\
\hline Average & 0.03 & 0.03 & 0.25 & 0.15 & 0.50 & 0.04 & 0.31 & 0.24 & 0.31 \\
\hline $\begin{array}{l}\text { Standard } \\
\text { Deviation }\end{array}$ & 0.01 & 0.01 & 0.05 & 0.03 & 0.08 & 0.01 & 0.03 & 0.01 & 0.02 \\
\hline
\end{tabular}

In similar fashion to peak pressure measurements, percent body weight postoperative results show a decrease in the toes (regions 1 and 2) and region 8. Although there is a dramatic shift laterally in the forefoot (region 5) and midfoot (region7), there is still an increase in the medial midfoot (region 6) which is disconcerting to the success of the surgery.

\section{Stance:}

Trials were taken with the aid of a chair for balance (if needed) for both two-foot stance as well as one-foot stance. For one-foot stance, both afflicted foot and un-afflicted foot were collected, however only the afflicted foot results are presented. Frame calibration was used to calibrate the trials by taking the middle frame of the collected response (10 seconds) for both two-foot and one-foot stance. Participant 1 did not have a pre-operative one-foot stance because the measurement had not yet been incorporated. 
Two-Foot Stance:

TWO-FOOT STANCE PEAK PRESSURE



Figure 23: Peak pressure measurements in each of the regions over total stance time with two-foot stance for the afflicted foot.

Pre-Op Peak Pressure (kPa):

\begin{tabular}{|l|r|r|r|r|r|r|r|r|r|}
\hline Regions & 1 & 2 & 3 & 4 & 5 & 6 & 7 & 8 & 9 \\
\hline & 12 & 23 & 40 & 25 & 30 & 18 & 13 & 89 & 68 \\
\hline
\end{tabular}

Post-Op Peak Pressure (kPa):

\begin{tabular}{|l|r|r|r|r|r|r|r|r|r|}
\hline Regions & 1 & 2 & 3 & 4 & 5 & 6 & 7 & 8 & 9 \\
\hline & 0 & 12 & 17 & 20 & 36 & 22 & 35 & 62 & 67 \\
\hline
\end{tabular}

Post-operative peak pressures for this participant show an increase in the midfoot, regions 6 and 7. This is particularly concerning because region 6 should show a decrease in pressure with surgical correction of flatfoot. There is a lateral shift in pressures in the forefoot from region 3 to region 5 , which demonstrates typical results post-operatively. 
TWO-FOOT STANCE PERCENT BODY WEIGHT

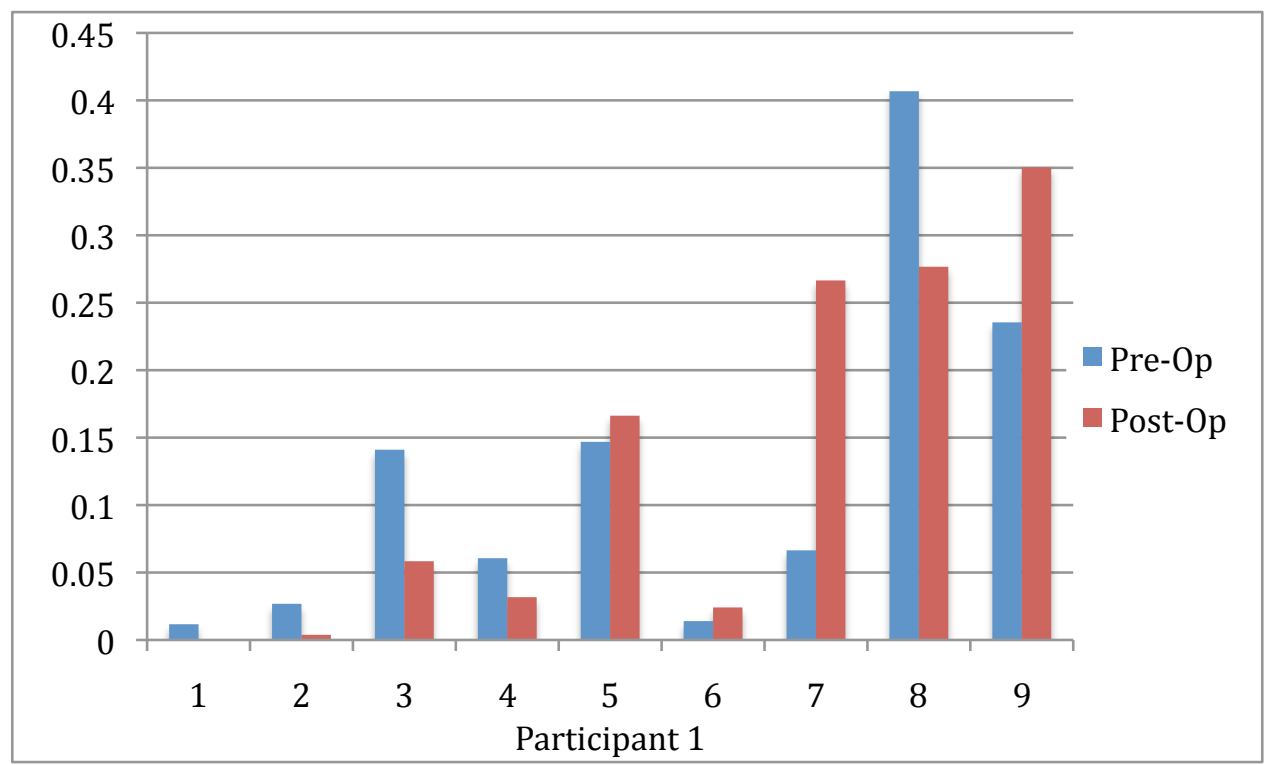

Figure 24: Percent body weight of two-foot stance in each region for the body weight distribution of afflicted foot

Pre-Op Percent Body Weight:

\begin{tabular}{|l|r|r|r|r|r|r|r|r|r|}
\hline Regions & 1 & 2 & 3 & 4 & 5 & 6 & 7 & 8 & 9 \\
\hline & 0.01 & 0.03 & 0.14 & 0.06 & 0.15 & 0.01 & 0.07 & 0.41 & 0.24 \\
\hline
\end{tabular}

Post-Op Percent Body Weight:

\begin{tabular}{|l|r|r|r|r|r|r|r|r|r|}
\hline Regions & 1 & 2 & 3 & 4 & 5 & 6 & 7 & 8 & 9 \\
\hline & 0.00 & 0.00 & 0.06 & 0.03 & 0.17 & 0.02 & 0.27 & 0.28 & 0.35 \\
\hline
\end{tabular}

Percent body weight shows a distribution where a lateral shift is observed in both the forefoot and midfoot upon surgical correction. Region 3 and 4 decrease in percent body weight where percent body weight increases in region 5 demonstrating the lateral correction. Although there is an increase in region 6, the dramatic increase in region 7 shows post-operative success where the participant's body weight is supported on the lateral midfoot. 


\section{Arch Index:}

Arch index was calculated using the frame where the area of contact was at peak during stance and walking trials after frame calibration. Calculations were made by using the formula from Murley et al by taking $\mathrm{B} /(\mathrm{A}+\mathrm{B}+\mathrm{C})$ with the exclusion of the toes.

TWO-FOOT/ONE-FOOT STANCE ARCH INDEX

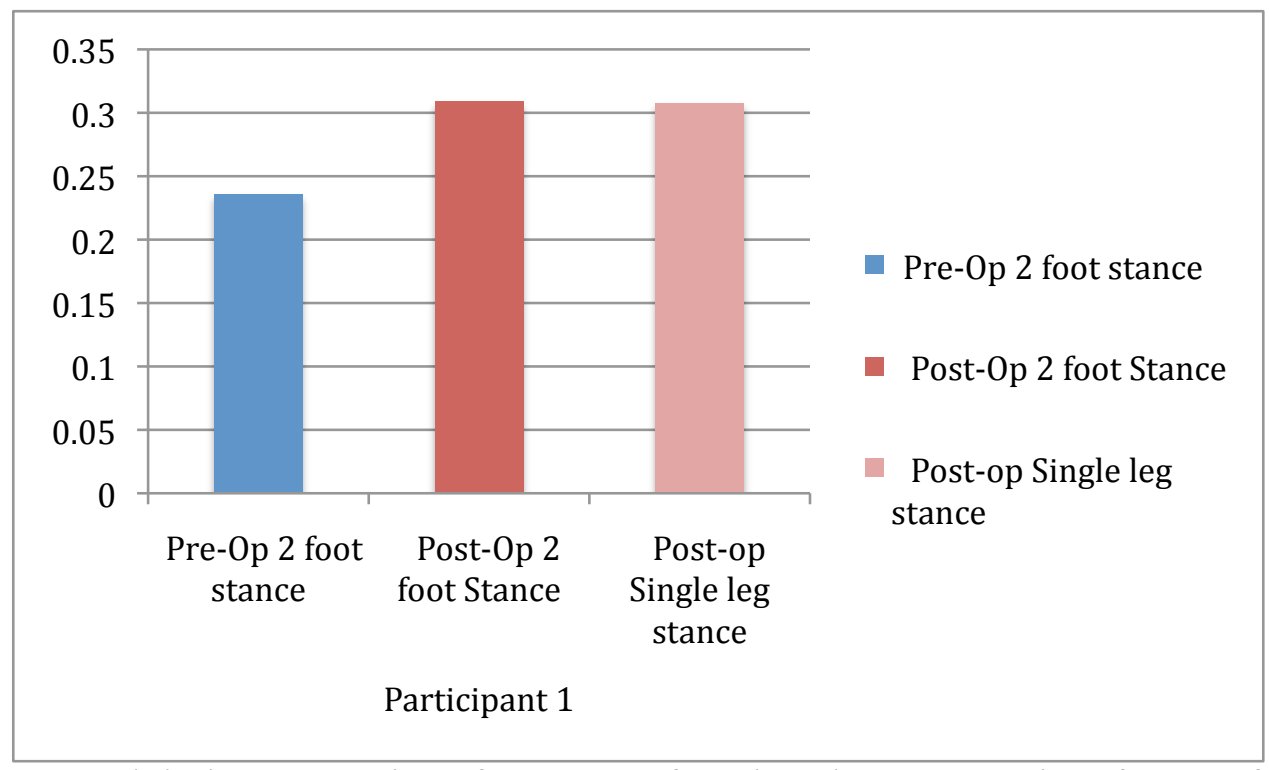

Figure 25: Arch index comparison for stance of total peak area over time for two-foot stance and one foot stance

Pre-Op Two-Foot Arch Index: 0.24

Post-Op Two-Foot Arch Index: 0.31

Post-Op One-Foot Arch Index: 0.31

Arch index post-operatively increased which would mean according to the measurement that the foot would get flatter. This may not be a valid measurement for participant 1's success of the surgery as most other measures showed success. 


\section{WALKING ARCH INDEX}

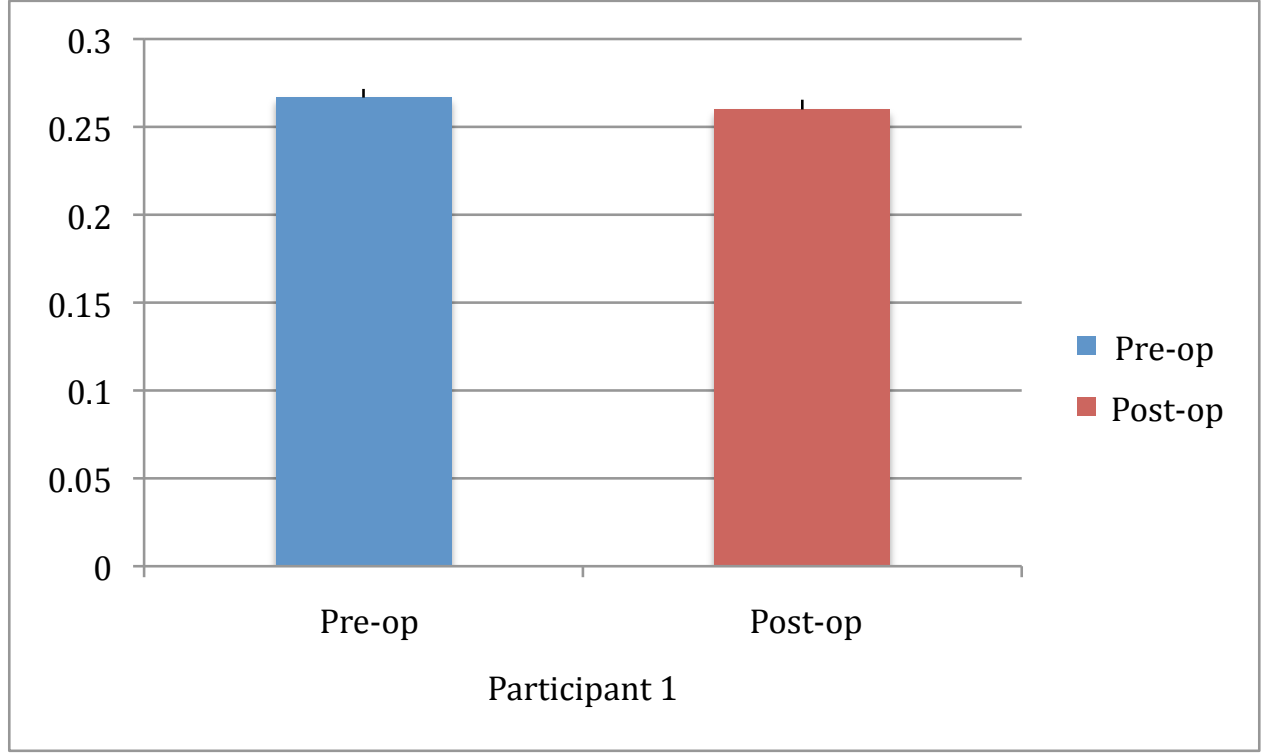

Figure 26: Arch index calculated from the walking plantar pressure profiles, averaged over three trials.

Pre-Op Walking Arch Index

\begin{tabular}{|r|r|r|r|r|r|r|}
\hline Forefoot & Midfoot & Hindfoot & \multicolumn{1}{l|}{ Total } & $\begin{array}{l}\text { Arch } \\
\text { Index }\end{array}$ & $\begin{array}{l}\text { Average } \\
\text { AI }\end{array}$ & STD Dev \\
\hline 49 & 28 & 31 & 109 & 0.26 & & \\
\hline 52 & 30 & 29 & 110 & 0.27 & & \\
\hline 50 & 29 & 29 & 108 & 0.27 & 0.27 & 0.01 \\
\hline
\end{tabular}

Post-Op Walking Arch Index

\begin{tabular}{|r|r|r|r|r|r|r|}
\hline Forefoot & Midfoot & Hindfoot & \multicolumn{1}{l|}{ Total } & $\begin{array}{l}\text { Arch } \\
\text { Index }\end{array}$ & $\begin{array}{l}\text { Average } \\
\text { AI }\end{array}$ & STD Dev \\
\hline 48 & 29 & 32 & 108 & 0.27 & & \\
\hline 51 & 29 & 34 & 114 & 0.25 & & \\
\hline 50 & 30 & 34 & 114 & 0.26 & 0.26 & 0.01 \\
\hline
\end{tabular}

The increase in weight (20lb ) may change the peak pressures seen postoperatively. It also may account for the increase in the arch index during standing due to the increase in force being put on the foot. Arch index measurements showed a surprising 
increase in area in the midfoot from normal values to a flatter index of 0.3 for the stance measurements but a decrease in the walking measurement of arch index when averaging the trials over time. The value of the walking arch index is very close to a normal average value of 0.24 (Murley, Menz, \& Landorf, 2009), therefore this participant shows success in the walking arch index measurement.

PARTICIPANT 5 (Right foot afflicted)

Participant 5 was first brought in on 3/14/2011 for plantar pressure analysis, radiographs, and motion analysis. Radiographs were taken first at third floor Gateway of MCV then the participant traveled to do motion analysis then plantar pressure protocol.

Participant 5 was brought back on 3/21/2012, 372 days after initial visit, for plantar pressure analysis and radiographs. No motion analysis was done. Plantar pressures were done in the Ambulatory Care Center in a spare protocol room. Radiographs were taken at third floor Gateway of MCV.

\section{Pre-Op Comments:}

The participant came in with a positive attitude to start testing. Motion analysis was performed first, where the participant walked approximately 25 times across the force-plate one way which may have increased her pain level. Although she sat between trials to complete the surveys given to her, she still had a hard time walking for the plantar pressure mat. Her gait pattern had one foot on for each walking trial recorded. Two-foot stance was performed as well as one-foot stance at a higher frequency to record more frames of data per second. 
Three trials of each walking analysis of each foot were performed, and when compared to normal trials, the participant was visibly flatfooted. The stance trials also showed visible midfoot collapse.

\section{Post- Op Comments:}

After surgical correction via a medial calcaneal osteotomy (MCO) and tendon transfer, the participant returned for testing. The participant came in with a cheerful attitude, and sat down to take the surveys. She joked and laughed when a question arose about doing "squats" on the sports part of the FAOS survey saying that she does not do any of these activities now. She also mentioned that she would be having upcoming knee surgery. Standing, she clearly favored one foot over the other, however did not completely utilize the chair in front of her for either two-foot or one-foot stance. She did have to do many more walking trials than pre-op because of the dragging of feet on the mat but she happily obliged with no rest between. Three walking trials were recorded for each foot. No motion analysis was done. Both two-foot and one-foot stance was captured and analyzed.

\section{Surveys:}

The SF-36 is a determinant of overall health and quality of life on a percent based scale where if the participant is closer to 100 then the general health is good.

$\underline{\text { SF-36 }}$

Pre-Op: 72

Post-Op: 90 
There was an increase in $18 \%$ of general health from pre-operative to a postoperative state. This increase accounts for physical well-being as well as emotional wellbeing. Participant 5 showed success of surgery in general health with an increase in this survey score.

The FAOS (foot and ankle outcome score) is a determinant of the health of the foot and ankle as well as scaling the level of activities of daily living and overall health due to foot and ankle problems. This scale is out of 500, where the higher the total score, the less affected the foot and ankle issue is to the participant.

\section{FAOS}

Pre-Op: 212

Post-Op: 475

There was a dramatic increase in FAOS scoring for participant 5. Pre-operatively participant 5 was functioning at $42 \%$ and increased post-operatively to $95 \%$. This increase is seen as a successful surgery with the participant returning to almost $100 \%$ functioning after surgical correction.

\section{Plantar pressure images:}

Participant 5's individual plantar pressure images are shown at peak pressure over time (Figures 27 and 28). These images show the maximum amount of firing on each sensel over total time on the plantar pressure mat. For the stance measurement the largest group of frame captures were used where the participant moved very little. The middle frame of the group was chosen as the most steady and used for calibration. Walking images shown are the total time it took for the participant to go from heel strike to toe off. 
PRE-OP PLANTAR PRESSURE IMAGES

\section{PARTICIPANT 5}



Figure 27: Pre-Op plantar pressure images for Participant 5 


\section{POST-OP PLANTAR PRESSURE IMAGES}

\section{PARTICIPANT 5 \\ $\underline{\mathrm{kPa}}$

Two-Foot Stance One-Foot Stance

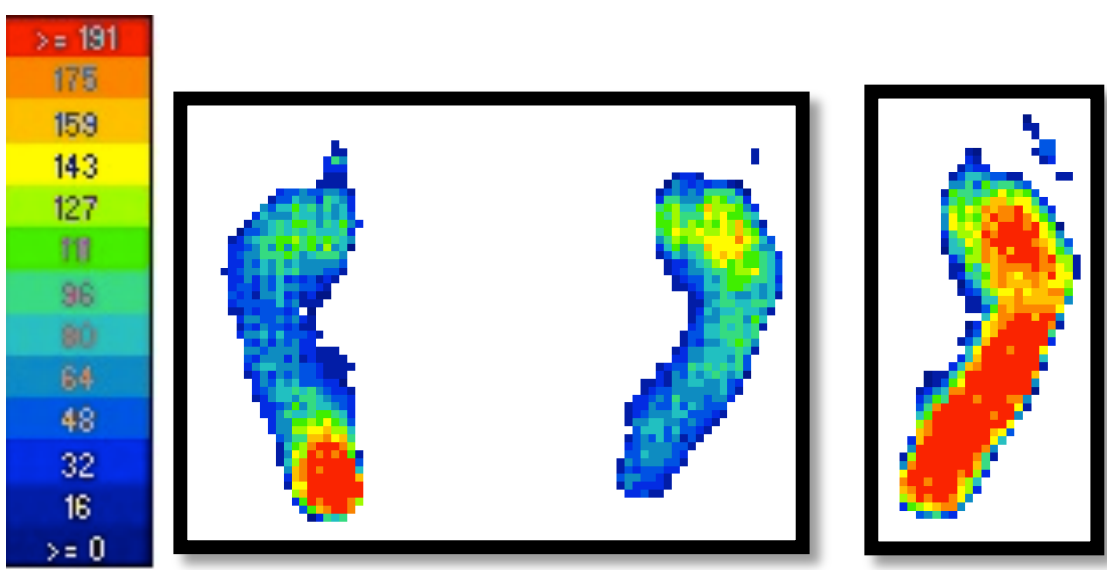

Walking
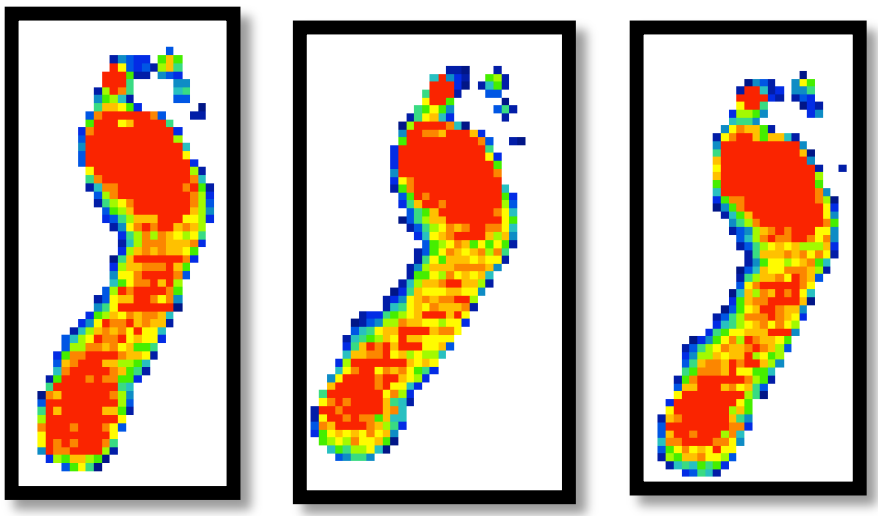

$\underline{\text { Trial } 1}$

Trial 2

$\underline{\text { Trial } 3}$

Figure 28: Post-Op plantar pressure images for Participant 5

There is a slight difference in the walking and stance pressures with more regions of red in post-operative images due to an increase in the weight of the participant postoperatively. It is to be noted that there is a visible decrease in the midfoot area after surgical correction where the foot takes on a more normal shape.

\section{Walking:}


Three trials of each foot for walking analysis was performed and compared to preop analysis. Calibration of the plantar pressure mat was done by frame calibration where for each walking trial the middle frame of midstance was used.

\section{Peak Pressure:}

Peak pressure is measured by taking the peak pressure in each region over the total time of the walking cycle. Each peak represents a distinct instant of time in the gait cycle for each region. This does not represent only one event in time, but nine separate events.

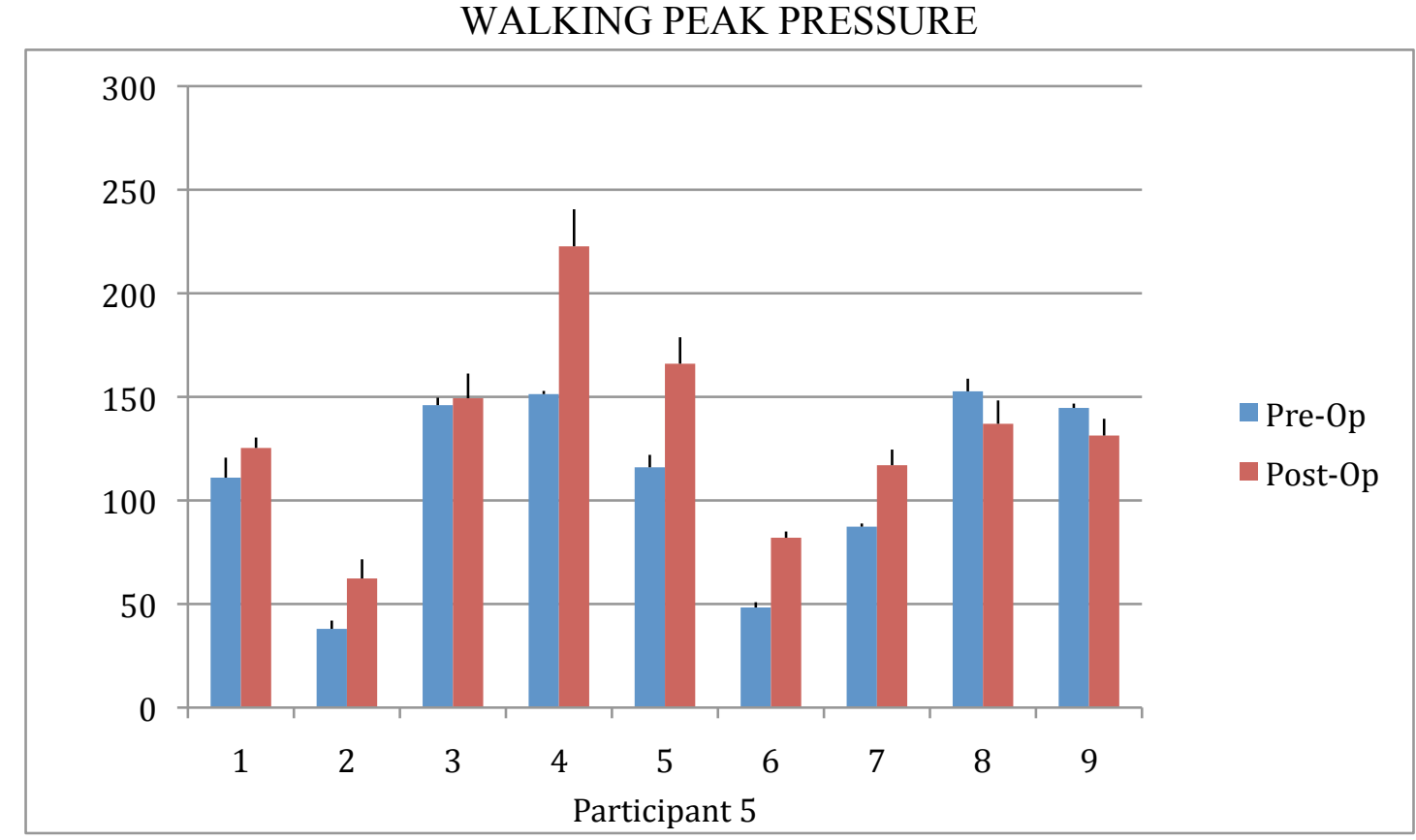

Figure 29: Peak pressure in each of the nine regions defined over the entire walking cycle averaged over three trials of the afflicted foot for Participant 5.

Pre-Op Peak Pressures $(\mathrm{kPa})$ :

\begin{tabular}{|l|l|l|l|l|l|l|l|l|l|}
\hline Regions & 1 & 2 & 3 & 4 & 5 & 6 & 7 & 8 & 9 \\
\hline
\end{tabular}




\begin{tabular}{|l|r|r|r|r|r|r|r|r|r|}
\hline Average & 111 & 38 & 146 & 151 & 116 & 48 & 87 & 153 & 145 \\
\hline $\begin{array}{l}\text { Standard } \\
\text { Deviation }\end{array}$ & 10 & 4 & 4 & 2 & 6 & 3 & 2 & 6 & 2 \\
\hline
\end{tabular}

Post-Op Peak Pressures (kPa):

\begin{tabular}{|l|r|r|r|r|r|r|r|r|r|}
\hline Regions & 1 & 2 & 3 & 4 & 5 & 6 & 7 & 8 & 9 \\
\hline Average & 125 & 62 & 149 & 223 & 166 & 82 & 117 & 137 & 131 \\
\hline $\begin{array}{l}\text { Standard } \\
\text { Deviation }\end{array}$ & 5 & 9 & 12 & 18 & 13 & 3 & 8 & 11 & 8 \\
\hline
\end{tabular}

Comparing the Pre-op pressures to the post-op pressures there is a general shift in pressure toward the front of the foot after surgery. Much of the pressure has been taken off the heel and been distributed through the forefoot and midfoot. Also a lateral shift can be observed, especially in regions 4 and 5 .

\section{Percent Body Weight:}

The measurement of percent body weight in each region was taken by the maximum force in pounds in that region on the afflicted foot and dividing it by the total body weight of the participant. This was done so that the measurements can be compared across time if the bodyweight of the participant changed. The participant was weighed both pre-operatively (204 lbs) and post-operatively (222.6 lbs). 


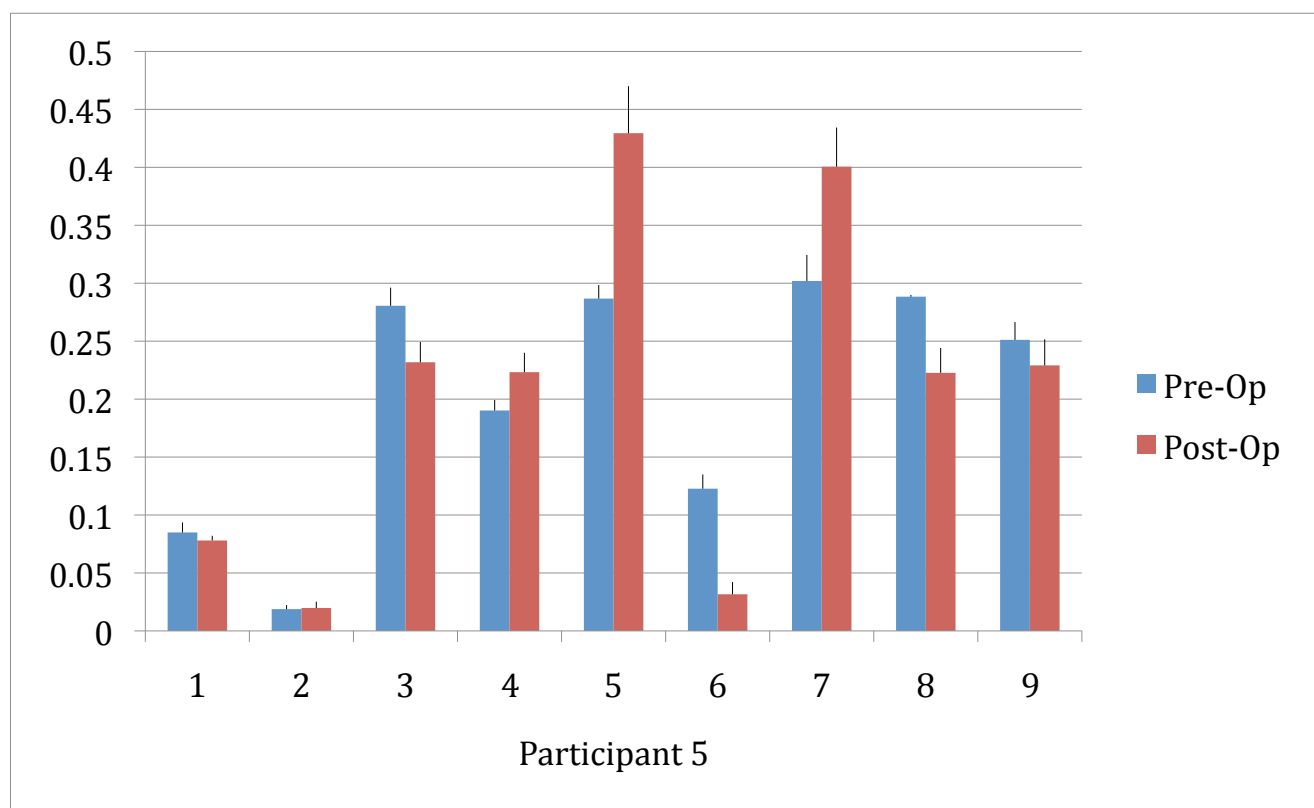

Figure 30: Maximum percent body weight in each of the nine regions over time of the walking trial for the average of three trials on the afflicted foot for Participant 5.

Pre-Op Percent Body Weight:

\begin{tabular}{|l|r|r|r|r|r|r|r|r|r|}
\hline Regions & 1 & 2 & 3 & 4 & 5 & 6 & 7 & 8 & 9 \\
\hline Average & 0.09 & 0.02 & 0.28 & 0.19 & 0.29 & 0.12 & 0.30 & 0.29 & 0.25 \\
\hline $\begin{array}{l}\text { Standard } \\
\text { Deviation }\end{array}$ & 0.01 & 0.00 & 0.02 & 0.01 & 0.01 & 0.01 & 0.02 & 0.00 & 0.02 \\
\hline
\end{tabular}

Post-Op Percent Body Weight:

\begin{tabular}{|l|r|r|r|r|r|r|r|r|r|}
\hline Regions & 1 & 2 & 3 & 4 & 5 & 6 & 7 & 8 & 9 \\
\hline Average & 0.08 & 0.02 & 0.23 & 0.22 & 0.43 & 0.03 & 0.40 & 0.22 & 0.23 \\
\hline $\begin{array}{l}\text { Standard } \\
\text { Deviation }\end{array}$ & 0.00 & 0.01 & 0.02 & 0.02 & 0.04 & 0.01 & 0.03 & 0.02 & 0.02 \\
\hline
\end{tabular}

Percent body weight measurement showed a lateral shift in regions 5 and 7 where more of the weight is being distributed laterally in both the forefoot and midfoot. Region 6 is of interest where the flatfoot has been corrected and is shown to have lessened the body weight being put on that region significantly, indicating a successful surgical correction. 


\section{Stance:}

Trials were taken with the aid of a chair for balance (if needed) for both two-foot stance as well as one-foot stance. For one-foot stance, both afflicted foot and un-afflicted foot were collected, however only the afflicted foot results are presented. Frame calibration was used to calibrate the trials by taking the middle frame of the collected response (10 seconds) for both two-foot and one-foot stance.

\section{Two-Foot Stance:}

TWO-FOOT STANCE PEAK PRESSURE

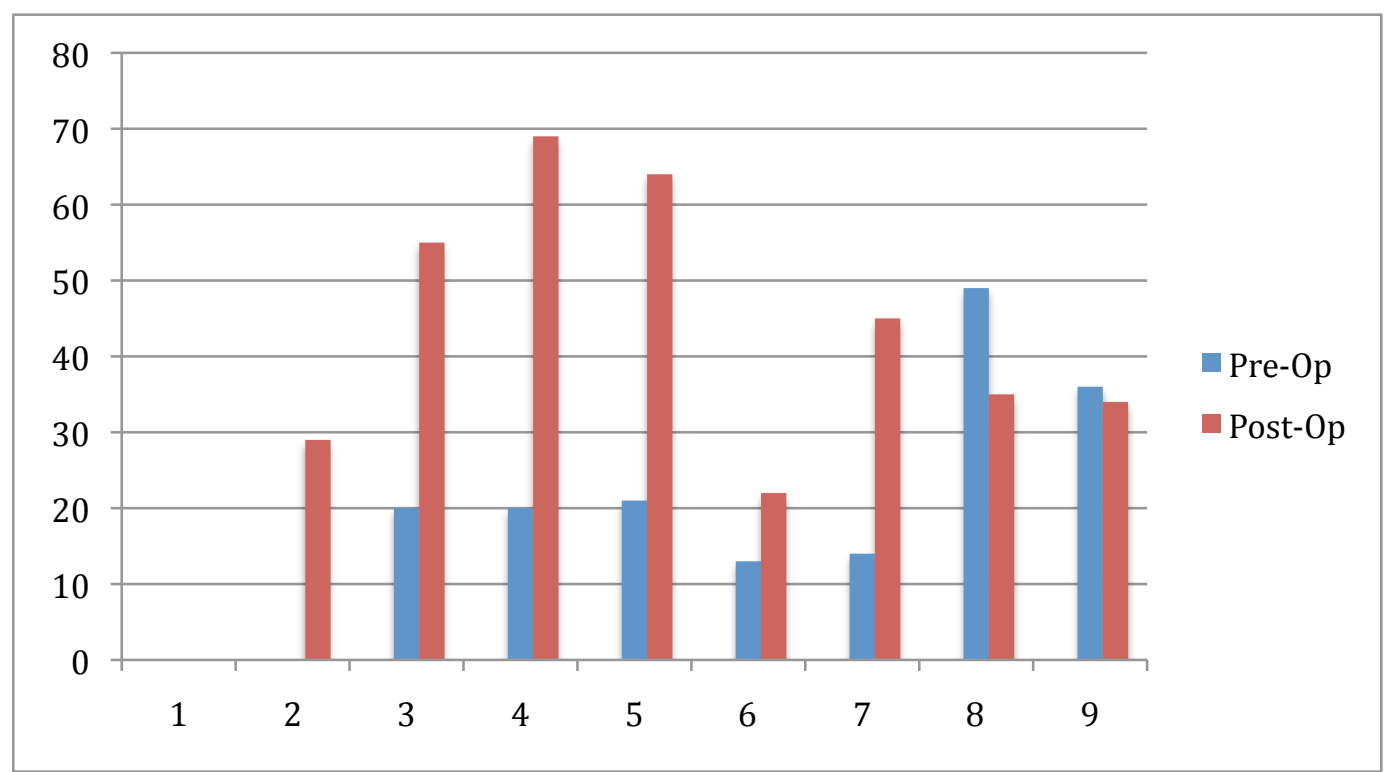

Figure 31: Peak pressure measurements in each of the regions over total stance time with two-foot stance for the afflicted foot

Pre-Op Pressure $(\mathrm{kPa})$ :

\begin{tabular}{|l|r|r|r|r|r|r|r|r|r|}
\hline Regions & 1 & 2 & 3 & 4 & 5 & 6 & 7 & 8 & 9 \\
\hline & 0 & 0 & 20 & 20 & 21 & 13 & 14 & 49 & 36 \\
\hline
\end{tabular}

Post-Op Pressure $(\mathrm{kPa})$ :

\begin{tabular}{|l|l|r|r|r|r|r|r|r|r|}
\hline Regions & 1 & 2 & 3 & 4 & 5 & 6 & 7 & 8 & 9 \\
\hline & 0 & 29 & 55 & 69 & 64 & 22 & 45 & 35 & 34 \\
\hline
\end{tabular}


In general, the pressure increased on two-foot stance in the post-op trial. This may be due to the fact that the participant is more comfortable putting weight on her afflicted foot after corrective surgery. In the post-op results there is more of a forefoot shift as well as lateral shift in the pressure readings as seen in the increases in regions 3 through 5. Although there is an increase in region 6, the medial midfoot, this may have occurred because of the increase in comfort to apply pressure.

TWO-FOOT STANCE PERCENT BODY WEIGHT

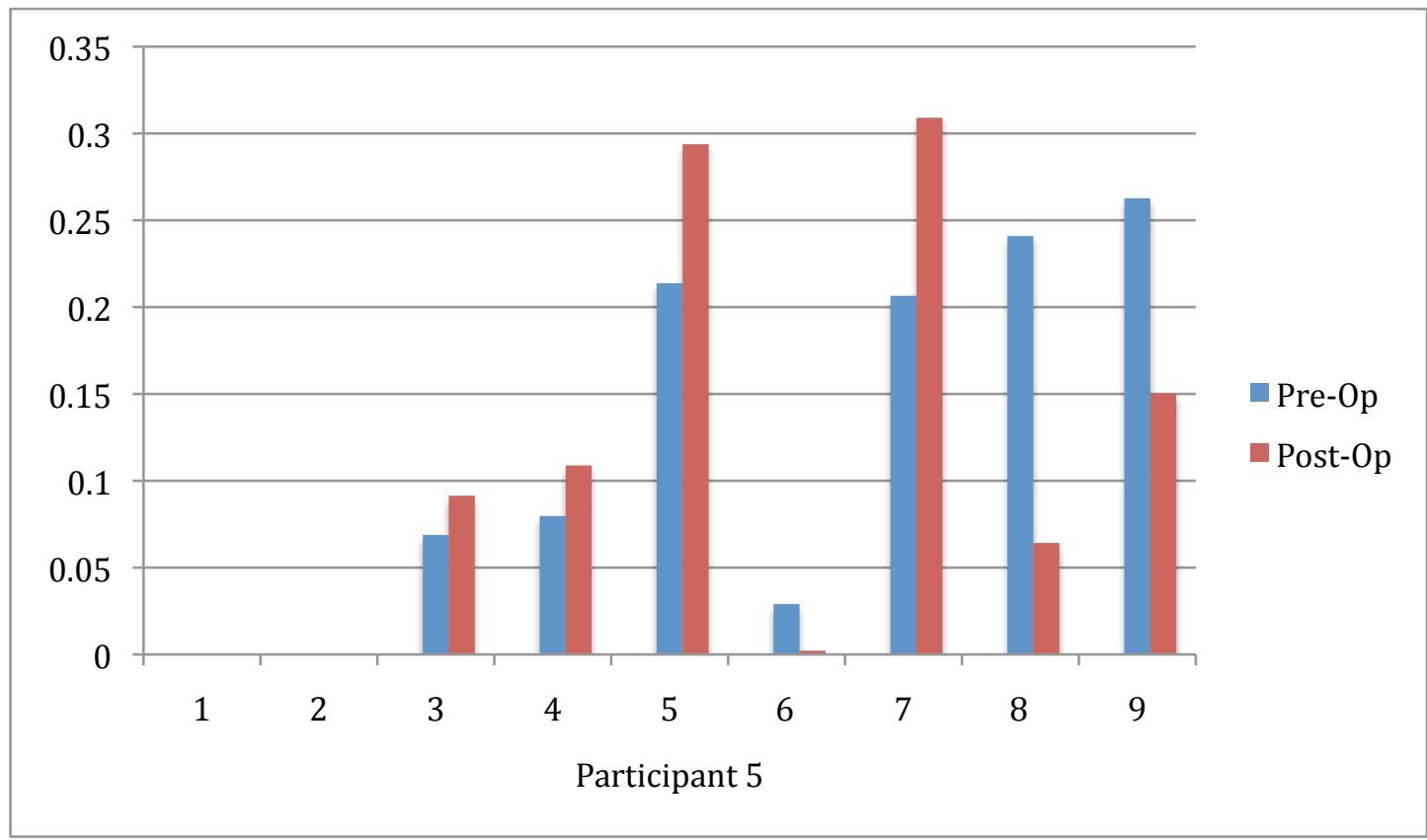

Figure 32: Percent body weight of two-foot stance for the body weight distribution of afflicted foot

Pre-Op Percent Body Weight:

\begin{tabular}{|l|r|r|r|r|r|r|r|r|r|}
\hline Regions & 1 & 2 & 3 & 4 & 5 & 6 & 7 & 8 & 9 \\
& 0.00 & 0.00 & 0.07 & 0.08 & 0.21 & 0.03 & 0.21 & 0.24 & 0.26 \\
\hline
\end{tabular}

Post-Op Percent Body Weight:

\begin{tabular}{|l|r|r|r|r|r|r|r|r|r|}
\hline Regions & 1 & 2 & 3 & 4 & 5 & 6 & 7 & 8 & 9 \\
\hline & 0.00 & 0.00 & 0.09 & 0.11 & 0.29 & 0.00 & 0.31 & 0.06 & 0.15 \\
\hline
\end{tabular}


Percent body weight shows a similar result to the peak pressure readings where the participant shows significant lateral shifting, especially in regions 5 and 7 (the lateral portion of the forefoot and midfoot respectively). The decrease in the medial midfoot (region 6) is a sign of successful surgery with arch correction. Both the increase in lateral shift and decrease in medial midfoot demonstrated surgical correction success.

\section{One-Foot Stance:}

\section{ONE FOOT STANCE PEAK PRESSURE}

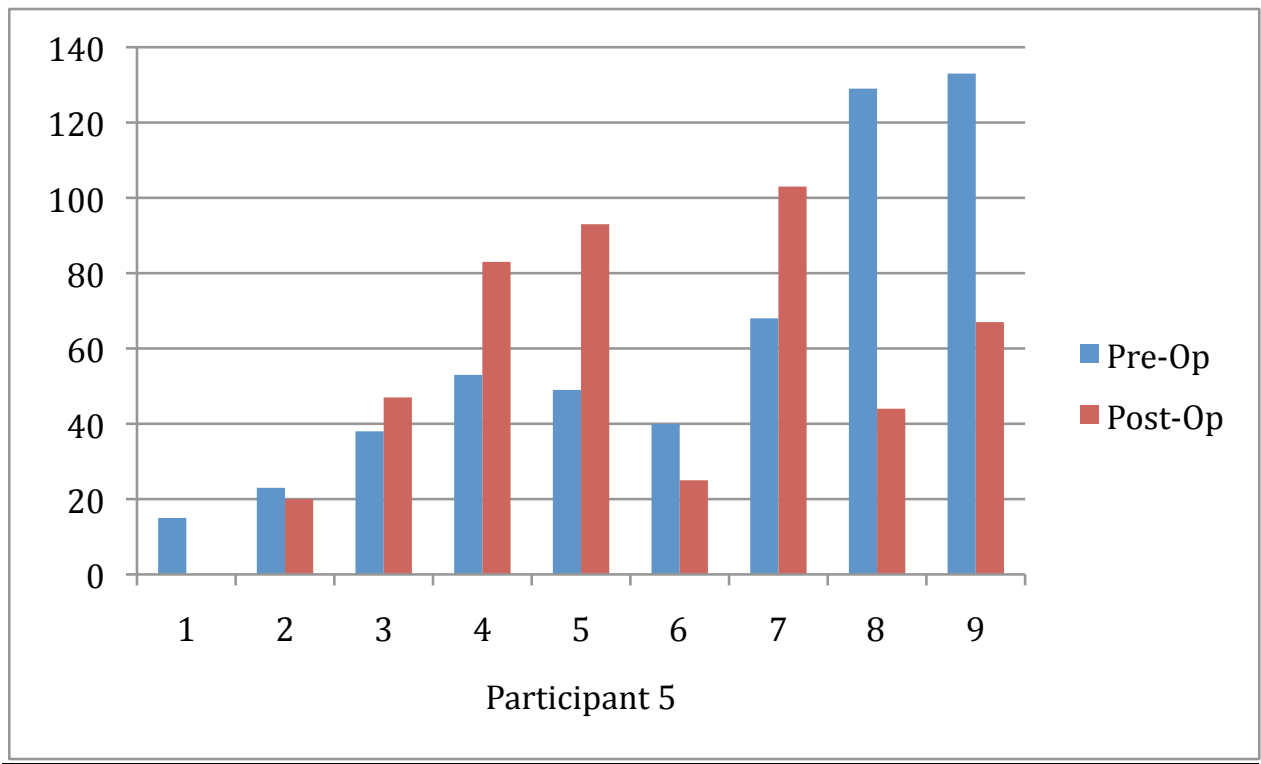

Figure 33: Peak pressure measurements in regions over total stance time with one-foot stance for the afflicted foot.

Pre-Op Pressure $(\mathrm{kPa})$ :

\begin{tabular}{|l|r|r|r|r|r|r|r|r|r|}
\hline Regions & 1 & 2 & 3 & 4 & 5 & 6 & 7 & 8 & 9 \\
\hline & 15 & 23 & 38 & 53 & 49 & 40 & 68 & 129 & 133 \\
\hline
\end{tabular}

Post-Op Pressure $(\mathrm{kPa})$ :

\begin{tabular}{|l|l|r|r|r|r|r|r|r|r|}
\hline Regions & 1 & 2 & 3 & 4 & 5 & 6 & 7 & 8 & 9 \\
\hline & 0 & 20 & 47 & 83 & 93 & 25 & 103 & 44 & 67 \\
\hline
\end{tabular}

As in two-foot stance, there is a lateral and forward shift of pressures where the hindfoot has less pressure in post-op results. This also may be due to the fact that when standing on one foot the participant was more likely to hold onto the chair for balance. 
This was the case for participant 5 holding on would create a forward shift in pressure.

The lateral shift with the increase in regions 5 and 7 can be explained by the correction of the flatfoot condition. A decrease in region 6 also demonstrates less pressure on the medial midfoot with surgical correction.

ONE FOOT STANCE PERCENT BODY WEIGHT



Figure 34: Percent body weight measurements in regions over total stance time with onefoot stance for the afflicted foot.

Pre- Op Percent Body Weight:

\begin{tabular}{|l|r|r|r|r|r|r|r|r|r|}
\hline Regions & 1 & 2 & 3 & 4 & 5 & 6 & 7 & 8 & 9 \\
\hline & 0.01 & 0.02 & 0.08 & 0.06 & 0.13 & 0.03 & 0.26 & 0.24 & 0.31 \\
\hline
\end{tabular}

Post-Op Percent Body Weight:

\begin{tabular}{|l|r|r|r|r|r|r|r|r|r|}
\hline Regions & 1 & 2 & 3 & 4 & 5 & 6 & 7 & 8 & 9 \\
\hline & 0.00 & 0.01 & 0.07 & 0.10 & 0.27 & 0.01 & 0.37 & 0.02 & 0.12 \\
\hline
\end{tabular}

Similar trends to one-foot pressure were seen in one-foot percent body weight. A lateral forward shift off the heels was seen. The largest change is in the region 7 demonstrating a lateral midfoot shift from pre-operative to post-operative state with the surgical correction. 


\section{Arch Index:}

Arch index was calculated using the frame where the area of contact was at a peak during stance and walking trials after frame calibration. Calculations were made by using the formula from Murley et al by taking $\mathrm{B} /(\mathrm{A}+\mathrm{B}+\mathrm{C})$ with the exclusion of the toes.

\section{TWO FOOT STANCE ARCH INDEX}

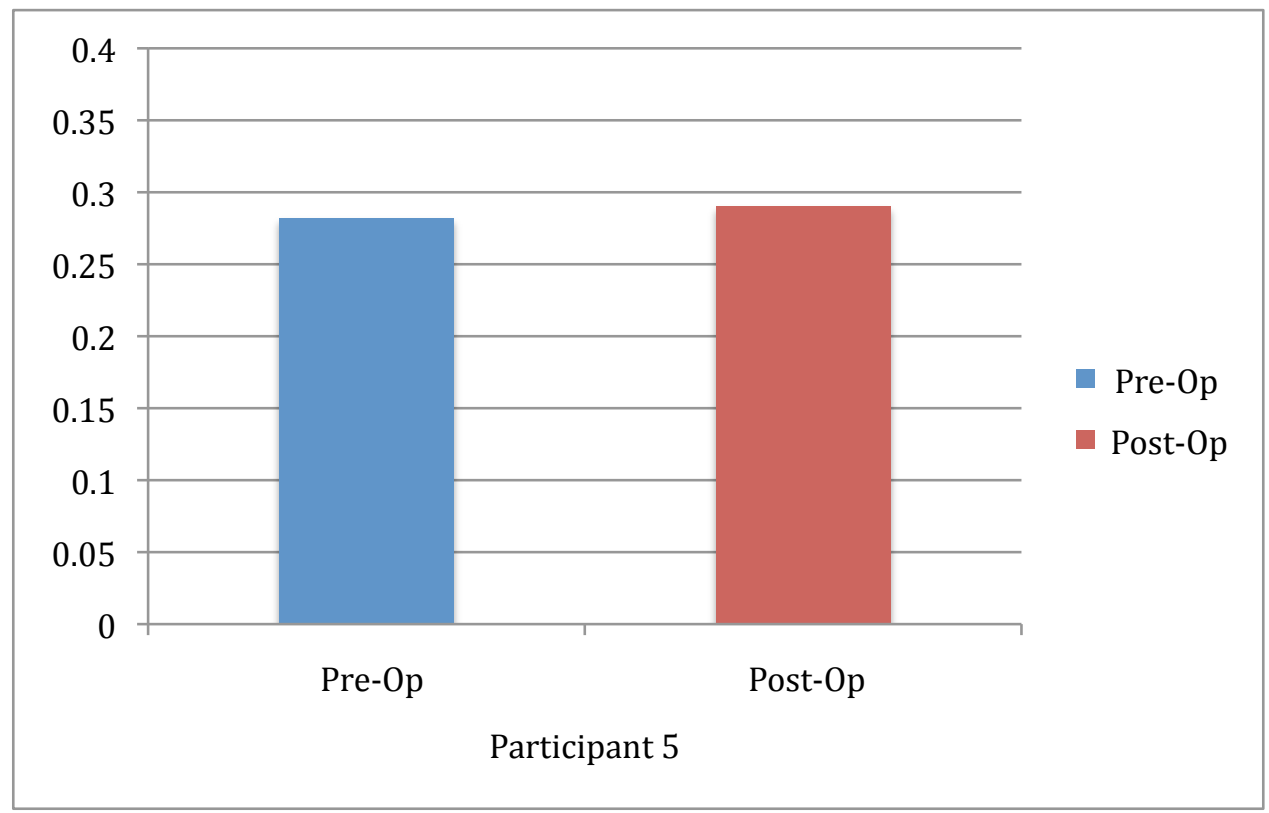

Figure 35: Arch index comparison for stance of total peak area over time for two-foot stance

Pre-Op Arch Index: 0.28

Post-Op Arch Index: 0.29 


\section{ONE FOOT ARCH INDEX}

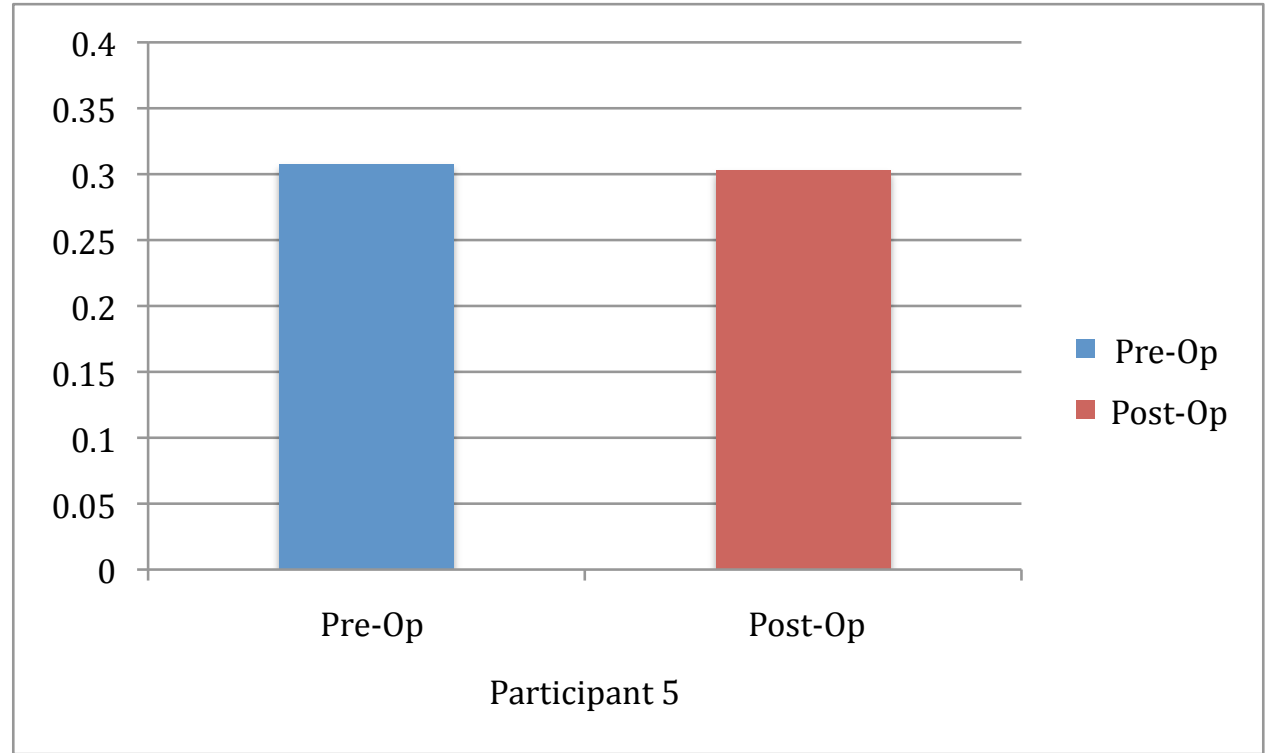

Figure 36: Arch index comparison for stance of total peak area over time for one foot stance.

Pre-Op Arch Index: 0.31

Post-Op Arch Index: 0.30

Although arch index measures are supposed to decrease to normal levels, this participant did not decrease to "normal" levels. However, this participant was AfricanAmerican and may have a higher arch index than normal levels which may account for the difference. Two-foot stance shows an increase in arch index and for one-foot stance a slight decrease. Arch index may not be a good indicator for this participant as to the progress made for pre-op and post-op comparison since there was very little change in both stance positions. 


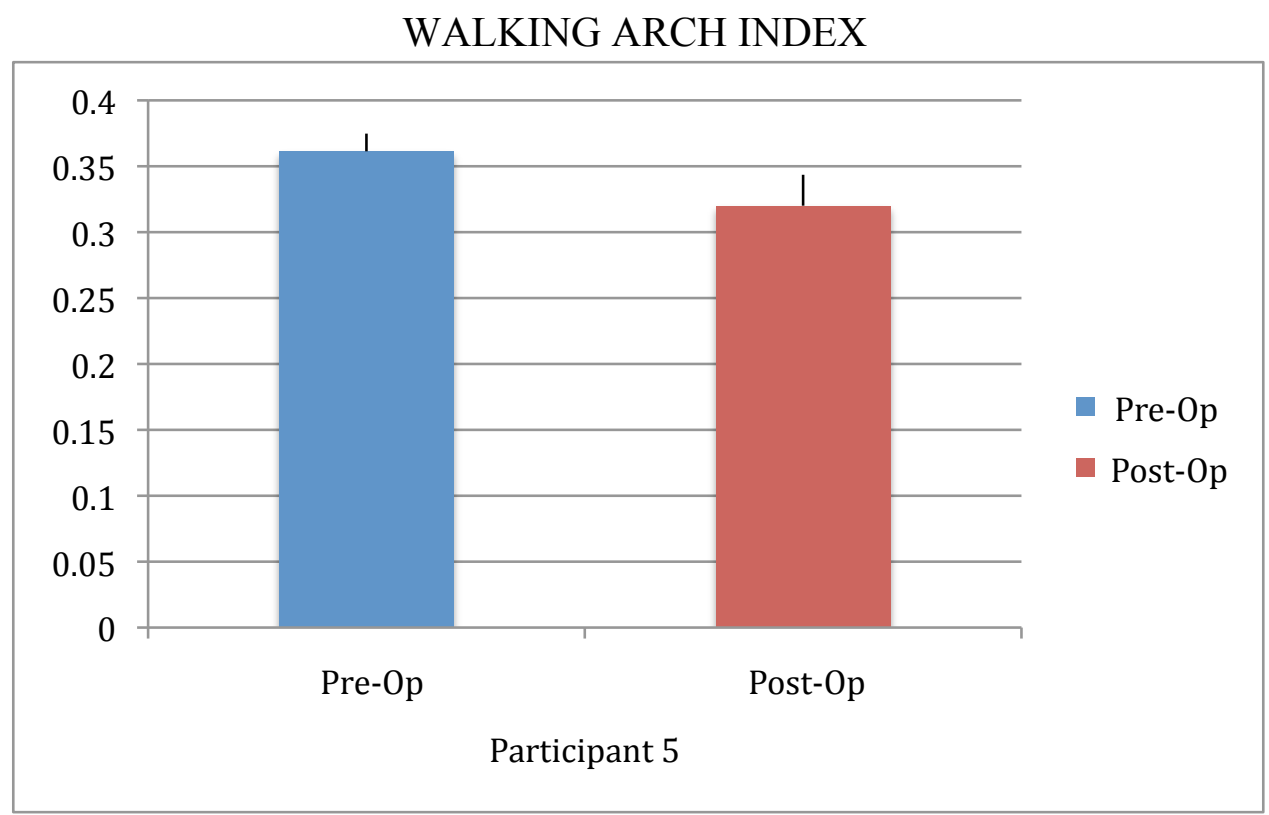

Figure 37: Arch index measurements of the peak areas over time of three walking trials.

Pre-Op Arch index:

\begin{tabular}{|r|r|r|r|r|r|r|}
\hline \multicolumn{1}{|l|}{ Forefoot } & \multicolumn{1}{|l|}{ Midfoot } & \multicolumn{1}{l|}{$\begin{array}{l}\text { Arch } \\
\text { Index }\end{array}$} & $\begin{array}{l}\text { Average } \\
\text { AI }\end{array}$ & STD Dev \\
\hline 58 & 57 & 38 & 153 & 0.37 & & \\
\hline 58 & 52 & 40 & 150 & 0.35 & & \\
\hline 61 & 56 & 36 & 153 & 0.37 & 0.36 & 0.01 \\
\hline
\end{tabular}

Post-Op Arch index:

\begin{tabular}{|r|r|r|r|r|r|r|}
\hline Forefoot & Midfoot & Hindfoot & Total & Arch Index & Average AI & STD Dev \\
\hline 50 & 44 & 35 & 129 & 0.34 & & \\
\hline 56 & 42 & 33 & 131 & 0.32 & & \\
\hline 55 & 38 & 36 & 129 & 0.30 & 0.32 & 0.02 \\
\hline
\end{tabular}

Arch index measures for walking decreased a significant amount from pre-op to post-op state. The arch index decreases if the foot arch support is better, and after surgical correction this participant demonstrates the decrease in walking arch index. Although still not at "normal" values the improvement in walking arch index shows promise for this participant. 


\section{GROUP RESULTS}

The overall success of surgery is difficult to quantify for a population, as there are many individual differences between each participant. . There were six total pre-op participants and five total post-op participants that scores were averaged and compared with a paired t-test. For plantar pressure measurements, each region was analyzed independently.

\section{Surveys:}

The SF-36 is a determinate of overall health and quality of life on a percent based scale where if the participant is closer to $100 \%$ then the general health is good.

\section{SF-36 SURVEY SCORES}

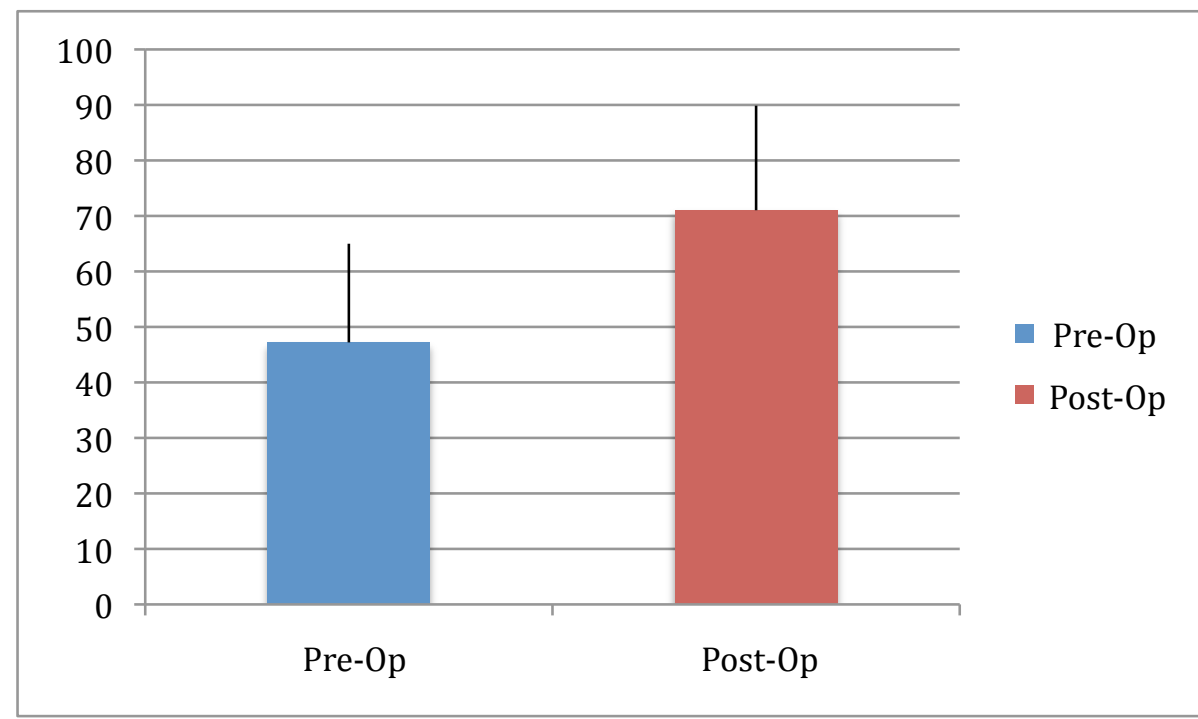

Figure 38: Average of total scores of the SF-36 general health survey for all participants Scores for SF-36 Survey (out of 100) 


\begin{tabular}{|l|l|l|l|l|l|l|c|c|}
\hline Participant & 1 & 2 & 3 & 4 & 5 & 6 & Average & STDev \\
\hline $\begin{array}{l}\text { Pre-Op } \\
\text { Score }\end{array}$ & 56 & 51 & 21 & 34 & 72 & 49 & 47 & 18 \\
\hline $\begin{array}{l}\text { Post-Op } \\
\text { Score }\end{array}$ & 79 & - & 84 & 55 & 90 & 47 & 71 & 19 \\
\hline
\end{tabular}

An overall increase $(\mathrm{p}<0.06)$ was observed post-operatively in the score of the SF-36 general health form except for participant 6 . Although this survey does not account for the foot and ankle directly, it does include physical functioning, pain and role limitations due to physical health. Emotional factors are also included which is indicative of overall perception of success of the surgery, which may have limited the overall increase of each participant's scoring.

The FAOS (foot and ankle outcome score) is a determinate of the health of the foot and ankle as well as scaling the level of activities of daily living and overall health due to foot and ankle problems. This scale is out of 500, where the higher the total score, the less affected the foot and ankle issue is to the participant.

FAOS SURVEY SCORES 


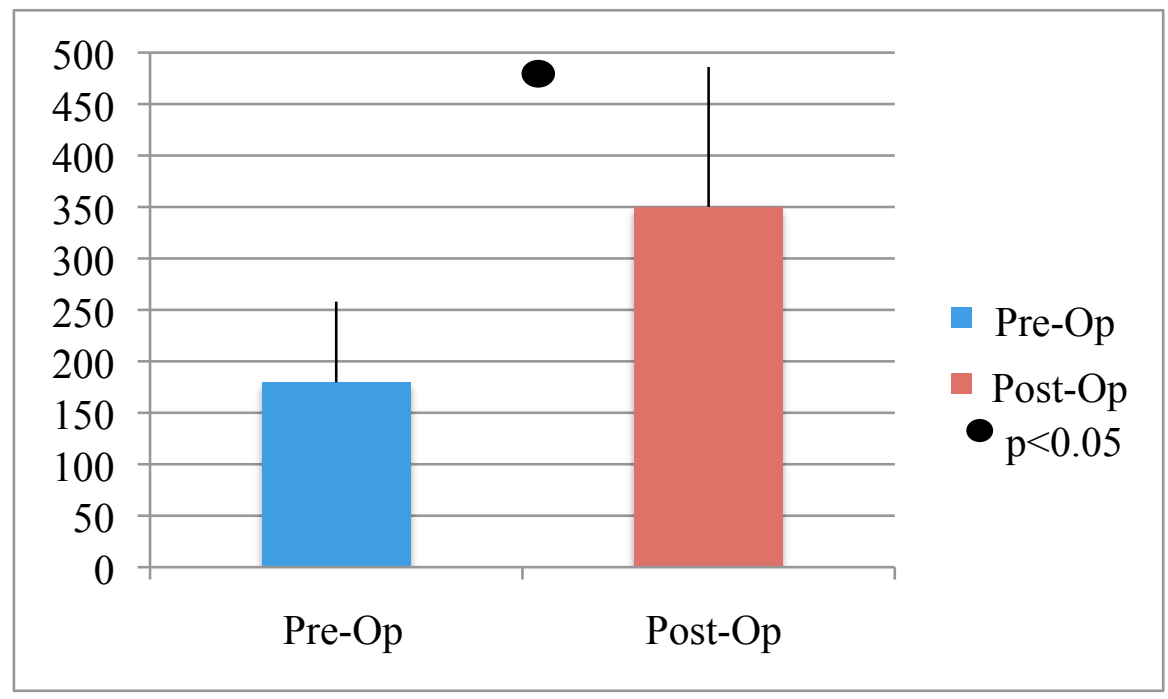

Figure 39: Total scores for the FAOS survey averaged with total number of scores FAOS Scores (out of 500)

\begin{tabular}{|l|l|l|l|l|l|r|r|r|}
\hline Participant & 1 & 2 & 3 & 4 & 5 & 6 & Average & STDev \\
\hline $\begin{array}{l}\text { Pre-Op } \\
\text { Score }\end{array}$ & 286 & 190 & 102 & 74 & 212 & 213 & 180 & 78 \\
\hline $\begin{array}{l}\text { Post-Op } \\
\text { Score }\end{array}$ & 449 & - & 413 & 163 & 475 & 251 & 350 & 136 \\
\hline
\end{tabular}

A dramatic increase in foot and ankle scores was seen after surgery in all participants. With a $p$ value of less than 0.05 , the FAOS scoring increase was significant in the success post-operatively. FAOS scoring accounts for activities of daily living and physical functioning of the ankle. Although participants may expect their foot and ankle to be similar to pre-injury after surgery, overall the increase in scoring means that participants were pleased with post-operative results.

With a total of six participants for the pre-operative analysis and five participants for the post-operative analysis, the success of surgical correction can be quantified through plantar pressure measurements with walking and stance. The measurements presented are the averages of the total pre-operative response and post-operative 
response. Standard deviations may be larger than customary for an in-vivo study because each participant was significantly different in their self-selected speed and cadence walking, and comfort-level with standing.

\section{Walking:}

Three trials of each foot for walking analysis was performed and when compared to pre-op analysis, there were some differences between percent body weight and pressure in the regions for both stance and walking as shown graphically below.

\section{Peak Pressure:}

This is measured by taking the total peak pressures over time. Each peak represents a different frame where the peak pressure in that region is shown graphically. This does not represent only one event in time, but nine separate events. The group result is the average of all six pre-op participants and five post-op participants.

WALKING PEAK PRESSURE 


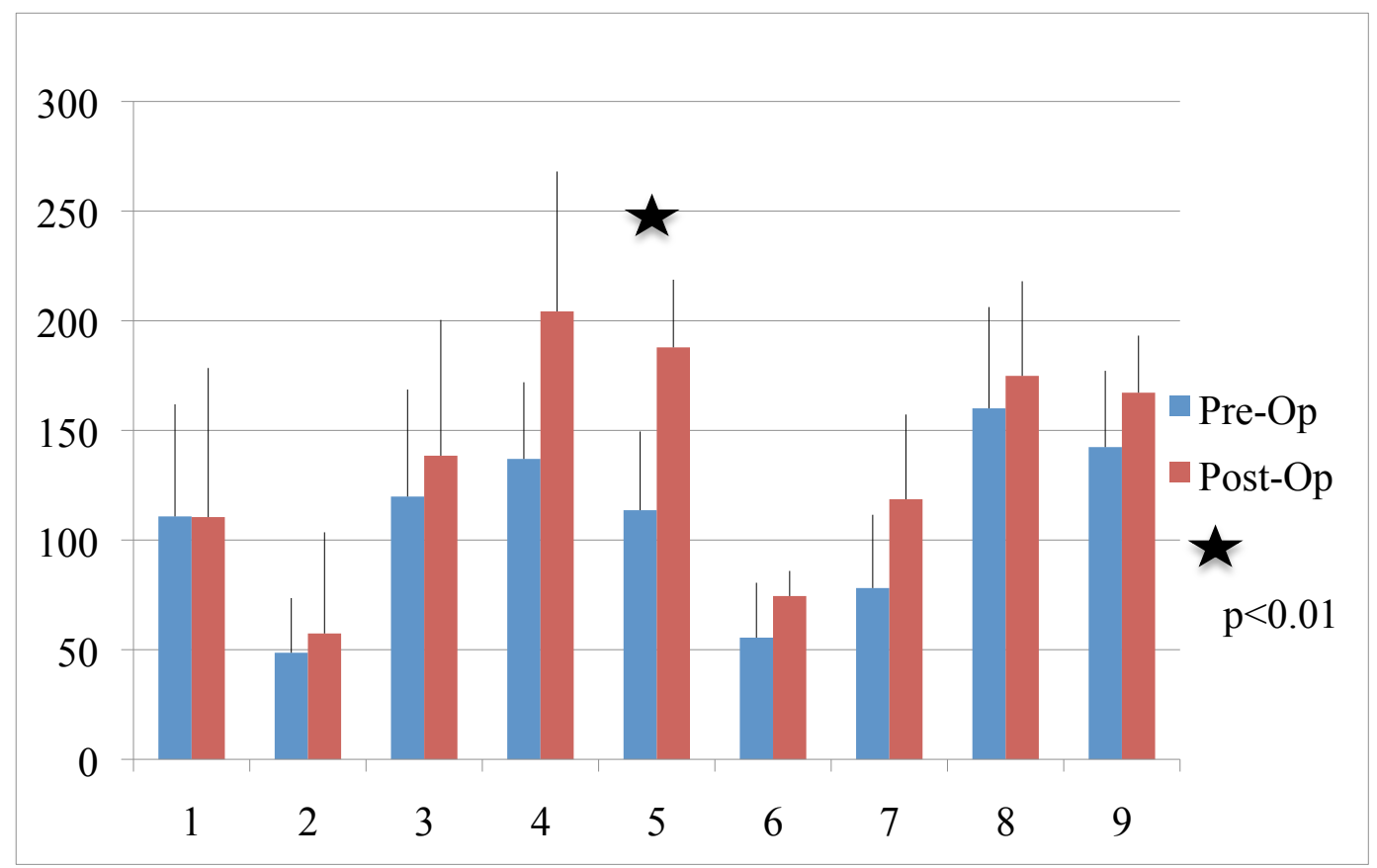

Figure 40: Peak pressure in the regions defined over the entire walking cycle for an average of 3 trials per participant and then the average of total trials for all participants.

Pre-Op Peak Pressures $(\mathrm{kPa})$ :

\begin{tabular}{|l|r|r|r|r|r|r|r|r|r|}
\hline Regions & 1 & 2 & 3 & 4 & 5 & 6 & 7 & 8 & 9 \\
\hline Average & 111 & 49 & 120 & 137 & 114 & 56 & 78 & 160 & 142 \\
\hline $\begin{array}{l}\text { Standard } \\
\text { Deviation }\end{array}$ & 51 & 25 & 49 & 35 & 36 & 25 & 33 & 46 & 35 \\
\hline
\end{tabular}

Post-Op Peak Pressures (kPa):

\begin{tabular}{|l|r|r|r|r|r|r|r|r|r|}
\hline Regions & 1 & 2 & 3 & 4 & 5 & 6 & 7 & 8 & 9 \\
\hline Average & 110 & 57 & 138 & 204 & 188 & 74 & 119 & 175 & 167 \\
\hline $\begin{array}{l}\text { Standard } \\
\text { Deviation }\end{array}$ & 68 & 46 & 62 & 64 & 31 & 11 & 39 & 43 & 26 \\
\hline
\end{tabular}

Although the walking peak pressure increased for each region post-operatively, the trend of a lateral shift is present in this graphical representation in the forefoot and midfoot. The forefoot with regions 3 through 5 demonstrates the lateral shift postoperatively with a larger shift in pressure from region 3 to regions 4 and 5 . The region 5 lateral shift from pre-op to post-op was deemed significant with the increase of pressure 
with a $\mathrm{p}$ value of less than 0.01 verifying the lateral shift in the forefoot after surgery.

Regions 6 and 7 of the midfoot show a lateral shift trend with region 7 increasing postoperatively. The hindfoot shows an equal distribution between medial and lateral heel peak pressures.

\section{Percent Body Weight:}

The measurement of percent body weight was taken by the force in pounds on the afflicted foot and dividing it by the total body weight of the participant. This was done so that the measurements can be compared across time if the bodyweight of the participant changed.

\section{WALKING PERCENT BODY WEIGHT}



Figure 41: Maximum percent body weight over time of the walking trial for the average of three trials per participant then averaged over total trials for all participants.

Pre- Op Percent Body Weight: 


\begin{tabular}{|l|r|r|r|r|r|r|r|r|r|}
\hline Regions & 1 & 2 & 3 & 4 & 5 & 6 & 7 & 8 & 9 \\
\hline Average & 0.09 & 0.04 & 0.25 & 0.16 & 0.28 & 0.09 & 0.23 & 0.29 & 0.28 \\
\hline $\begin{array}{l}\text { Standard } \\
\text { Deviation }\end{array}$ & 0.06 & 0.03 & 0.12 & 0.05 & 0.07 & 0.07 & 0.08 & 0.06 & 0.07 \\
\hline
\end{tabular}

Post- Op Percent Body Weight:

\begin{tabular}{|l|r|r|r|r|r|r|r|r|r|}
\hline Regions & 1 & 2 & 3 & 4 & 5 & 6 & 7 & 8 & 9 \\
\hline Average & 0.07 & 0.04 & 0.21 & 0.18 & 0.48 & 0.06 & 0.37 & 0.27 & 0.30 \\
\hline $\begin{array}{l}\text { Standard } \\
\text { Deviation }\end{array}$ & 0.05 & 0.03 & 0.11 & 0.03 & 0.09 & 0.04 & 0.11 & 0.04 & 0.04 \\
\hline
\end{tabular}

Percent body weight is a standardized measure for each participant that shows the trends of a successful surgical correction. In the forefoot, a post-operative decrease in region 3 and a significant increase in region 5 accounts for the shift in body weight from medial to lateral. With a $\mathrm{p}$ value of less than 0.01 , region 5 shows that this finding is noteworthy for the correction of flatfoot in these participants. In the midfoot, a postoperative decrease in region 6 and a significant increase in region 7 demonstrate the shift of body weight from medial to lateral. With a $\mathrm{p}$ value of less than 0.05 , region 7 illustrates the correction of the midfoot. The midfoot is particularly important as all of the participants had low or no arch. The hindfoot is roughly equal from medial to lateral in both pre-operative and post-operative states.

\section{Stance:}

Trials were taken with the aid of a chair for balance (if needed) for both two-foot stance as well as one-foot stance. For one-foot stance, both afflicted foot and un-afflicted foot were collected, however only the afflicted foot results are presented. Frame calibration was used to calibrate the trials by taking the middle frame of the collected response (10 seconds). 
Two-Foot Stance:

TWO-FOOT STANCE PEAK PRESSURE

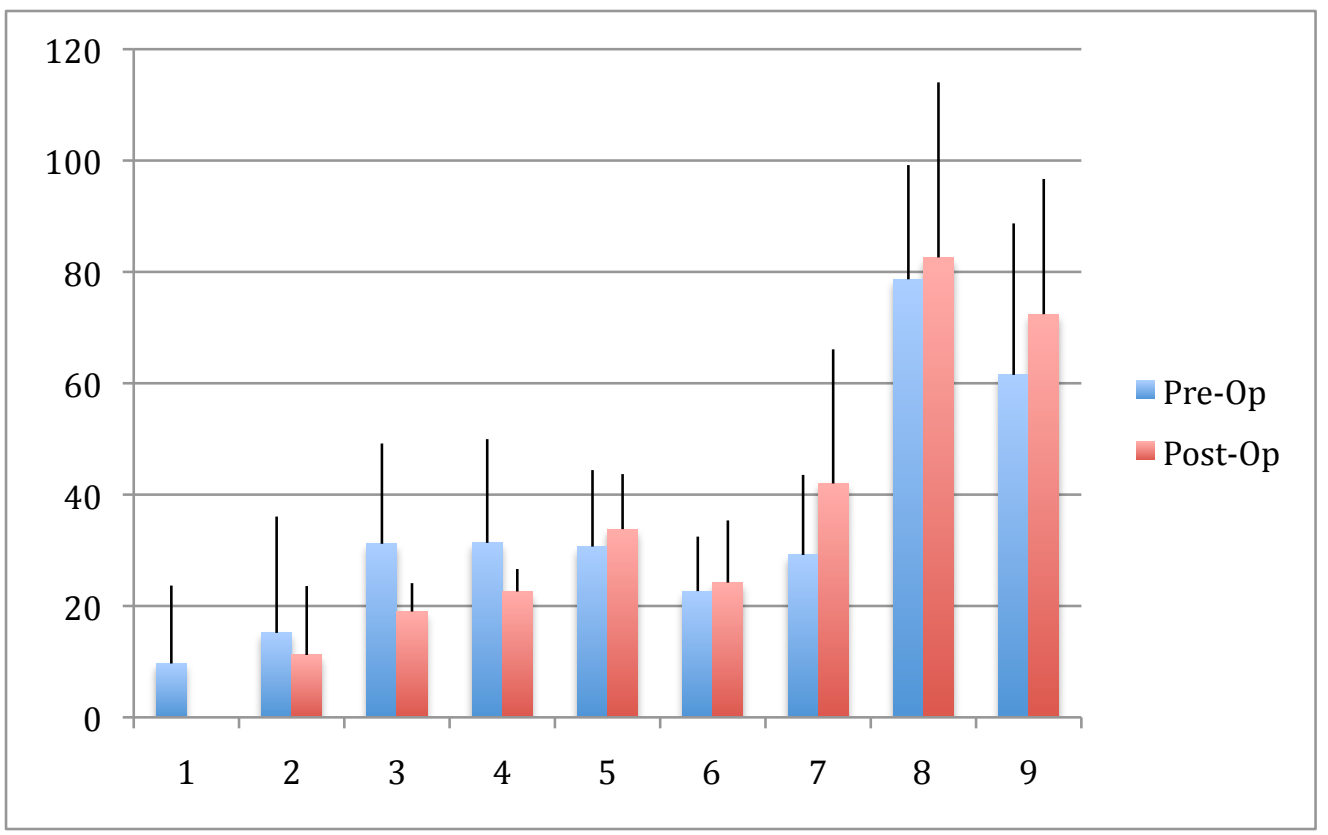

Figure 42: Peak pressure over time of the nine regions of the walking trial averaged over total trials for all participants for two-foot stance. This measurement does not measure both feet, but afflicted foot only.

Pre-Op Peak Pressure (kPa):

\begin{tabular}{|l|r|r|r|r|r|r|r|r|r|}
\hline Regions & 1 & 2 & 3 & 4 & 5 & 6 & 7 & 8 & 9 \\
\hline Average & 10 & 15 & 31 & 31 & 31 & 23 & 29 & 79 & 62 \\
\hline $\begin{array}{l}\text { Standard } \\
\text { Deviation }\end{array}$ & 14 & 21 & 18 & 19 & 14 & 10 & 14 & 21 & 27 \\
\hline
\end{tabular}

Post-Op Peak Pressure (kPa):

\begin{tabular}{|l|r|r|r|r|r|r|r|r|r|}
\hline Regions & 1 & 2 & 3 & 4 & 5 & 6 & 7 & 8 & 9 \\
\hline Average & 0 & 11 & 19 & 23 & 34 & 24 & 42 & 83 & 72 \\
\hline $\begin{array}{l}\text { Standard } \\
\text { Deviation }\end{array}$ & 0 & 12 & 5 & 4 & 10 & 11 & 24 & 31 & 24 \\
\hline
\end{tabular}

Peak pressure measurement did not have any significant results, however region 3 decreased a small amount post-operatively and a small increase in region 5 displays a lateral shift in pressure. The other regions do not show significant trends, with p-values ranging from 0.16 to 0.81 over the nine regions. 
TWO-FOOT STANCE PERCENT BODY WEIGHT

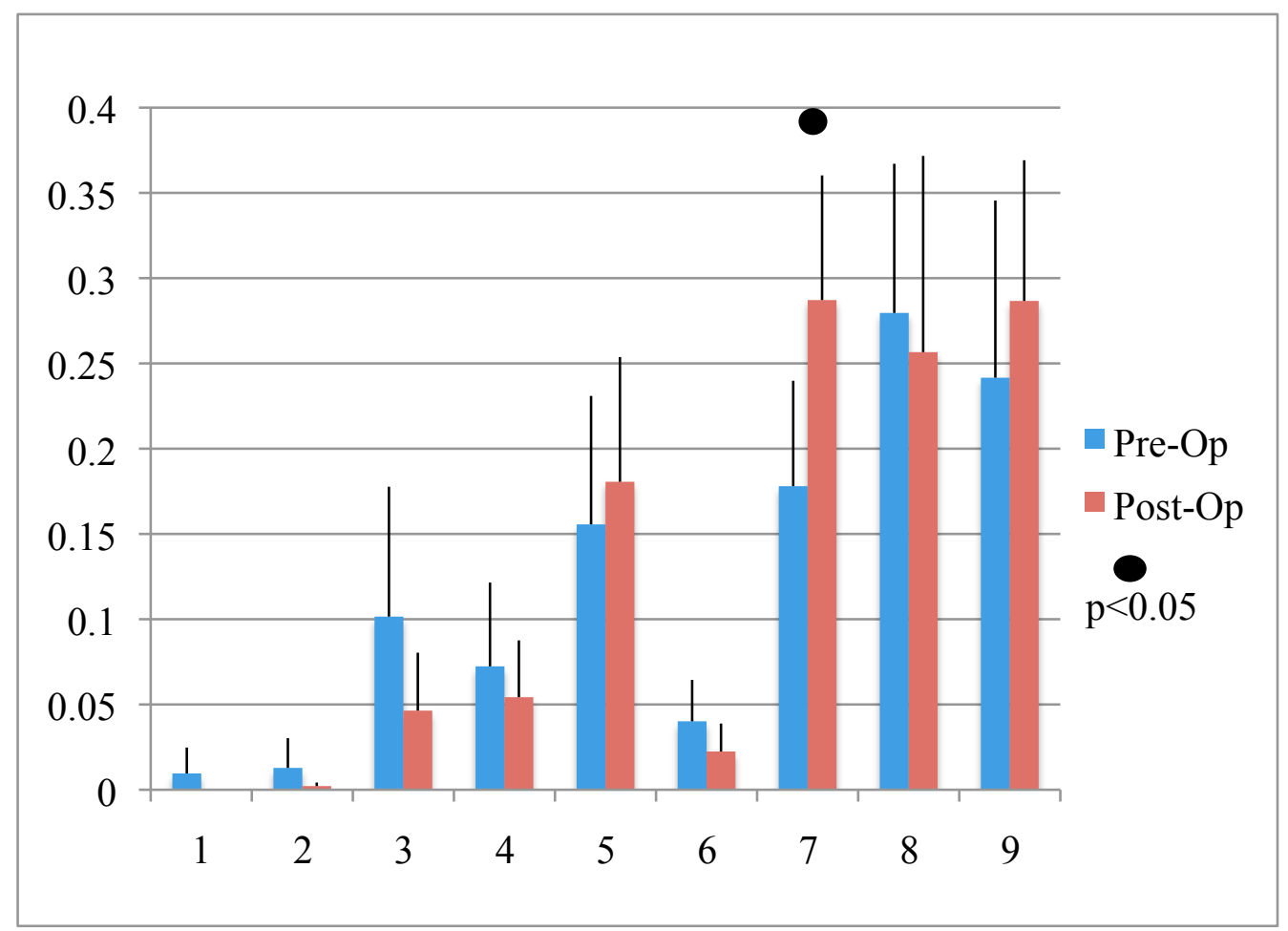

Figure 43: Maximum percent body weight over time of the nine regions of the walking trial averaged over total trials for all participants in two-foot stance. This measurement does not measure both feet, but afflicted foot only.

Pre- Op Percent Body Weight:

\begin{tabular}{|l|r|r|r|r|r|r|r|r|r|}
\hline Regions & 1 & 2 & 3 & 4 & 5 & 6 & 7 & 8 & 9 \\
\hline Average & 0.01 & 0.01 & 0.10 & 0.07 & 0.16 & 0.04 & 0.18 & 0.28 & 0.24 \\
\hline $\begin{array}{l}\text { Standard } \\
\text { Deviation }\end{array}$ & 0.01 & 0.02 & 0.08 & 0.05 & 0.07 & 0.02 & 0.06 & 0.09 & 0.10 \\
\hline
\end{tabular}

Post- Op Percent Body Weight:

\begin{tabular}{|l|r|r|r|r|r|r|r|r|r|}
\hline Regions & 1 & 2 & 3 & 4 & 5 & 6 & 7 & 8 & 9 \\
\hline Average & 0.00 & 0.00 & 0.05 & 0.05 & 0.18 & 0.02 & 0.29 & 0.26 & 0.29 \\
\hline $\begin{array}{l}\text { Standard } \\
\text { Deviation }\end{array}$ & 0.00 & 0.00 & 0.03 & 0.03 & 0.07 & 0.02 & 0.07 & 0.12 & 0.08 \\
\hline
\end{tabular}

As seen in walking percent body weight, two-foot stance also showed similar trends from a medial aspect to lateral aspect post-operatively. In the forefoot, there is a trend 
with a post-operative increase in region 5, the lateral portion and a decrease in region 3 the medial portion of the foot with p-values ranging from 0.02 to 0.59 over the nine regions. In the midfoot, there is a significant increase in region 7 with a $p$ value of less than 0.05 , which means that there is a lateral shift in bodyweight. Region 6 also decreases which would suggest the surgical correction was a success with more of an arch. The hindfoot is equal in the medial and lateral portions from pre-op to post-op states.

One-foot stance:

It is to be noted that although there are six total pre-operative participants, that only three participants were measured for one-foot stance because the measurement was not incorporated into the study at first. Post-operatively there are five participants. One-foot stance may vary because of the use of the chair in front of the participant.

ONE FOOT STANCE PEAK PRESSURE



Figure 44: Peak pressure over time of the nine regions of the walking trial averaged over total trials for all participants for one-foot stance.

Pre-Op Peak Pressure:

\begin{tabular}{|l|l|l|l|l|l|l|l|l|r|}
\hline Regions & 1 & 2 & 3 & 4 & 5 & 6 & 7 & 8 & 9 \\
\hline
\end{tabular}




\begin{tabular}{|l|r|r|r|r|r|r|r|r|r|}
\hline Average & 30 & 12 & 35 & 43 & 58 & 54 & 88 & 130 & 136 \\
\hline $\begin{array}{l}\text { Standard } \\
\text { Deviation }\end{array}$ & 39 & 12 & 34 & 39 & 8 & 28 & 45 & 46 & 53 \\
\hline
\end{tabular}

Post-Op Peak Pressure

\begin{tabular}{|l|r|r|r|r|r|r|r|r|r|}
\hline Regions & 1 & 2 & 3 & 4 & 5 & 6 & 7 & 8 & 9 \\
\hline Average & 29 & 28 & 51 & 59 & 78 & 44 & 113 & 112 & 121 \\
\hline $\begin{array}{l}\text { Standard } \\
\text { Deviation }\end{array}$ & 55 & 36 & 36 & 23 & 21 & 18 & 44 & 41 & 33 \\
\hline
\end{tabular}

There were no significant results for one-foot stance where p-values ranged from 0.18 to 0.99 over the nine regions. This may be due to a high variance in standing and balance between participants. Also holding onto the chair may have skewed regions, which could account for the differences between the regions and high standard deviations between participants. Since one-foot stance is hard to balance for 10 seconds without swaying this may also have an effect of this results.

\section{ONE FOOT STANCE PERCENT BODY WEIGHT}

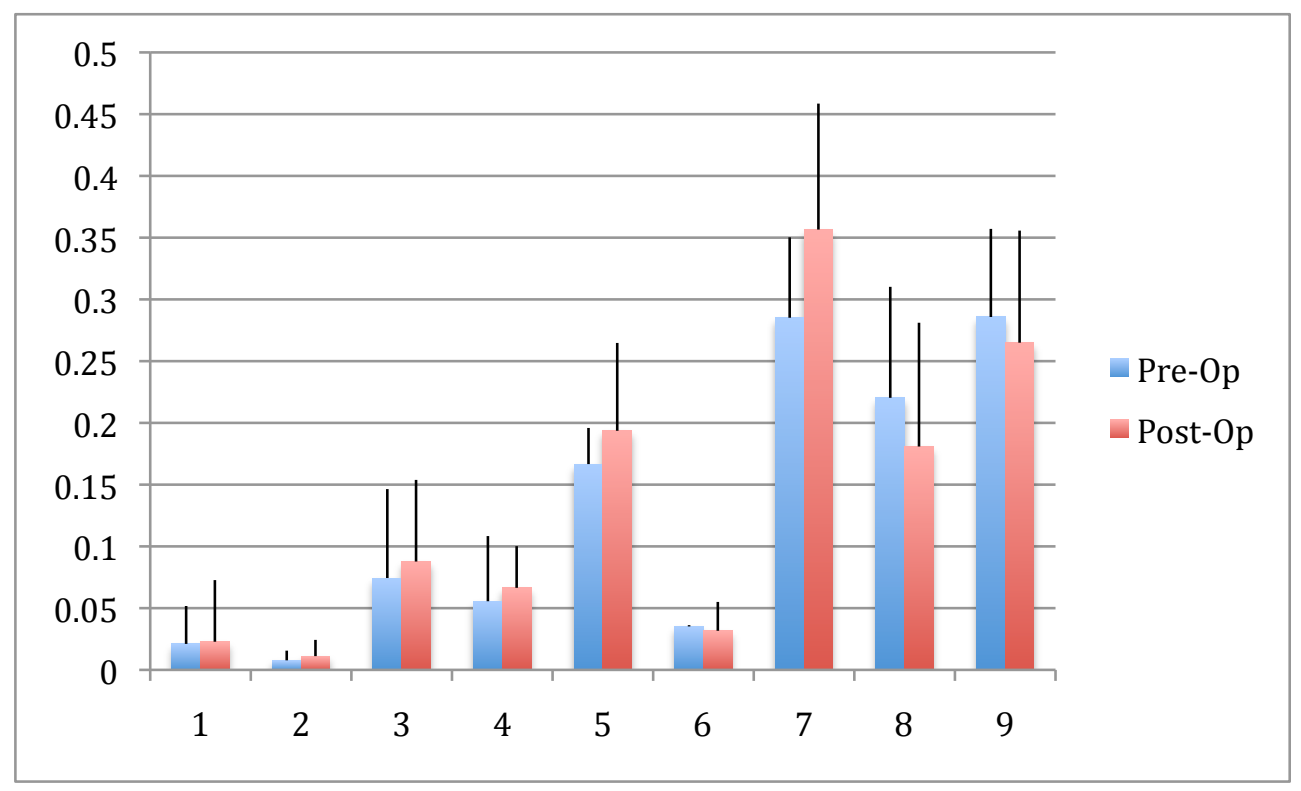

Figure 45: Maximum percent body weight over time of the nine regions of the walking trial averaged over total trials for all participants for one-foot stance.

Pre-Op Percent Body Weight: 


\begin{tabular}{|l|r|r|r|r|r|r|r|r|r|}
\hline Regions & 1 & 2 & 3 & 4 & 5 & 6 & 7 & 8 & 9 \\
\hline Average & 0.02 & 0.01 & 0.07 & 0.06 & 0.17 & 0.04 & 0.29 & 0.22 & 0.29 \\
\hline $\begin{array}{l}\text { Standard } \\
\text { Deviation }\end{array}$ & 0.03 & 0.01 & 0.07 & 0.05 & 0.03 & 0.00 & 0.07 & 0.09 & 0.07 \\
\hline
\end{tabular}

Post-Op Percent Body Weight:

\begin{tabular}{|l|r|r|r|r|r|r|r|r|r|}
\hline Regions & 1 & 2 & 3 & 4 & 5 & 6 & 7 & 8 & 9 \\
\hline Average & 0.02 & 0.01 & 0.09 & 0.07 & 0.19 & 0.03 & 0.36 & 0.18 & 0.26 \\
\hline $\begin{array}{l}\text { Standard } \\
\text { Deviation }\end{array}$ & 0.05 & 0.01 & 0.07 & 0.03 & 0.07 & 0.02 & 0.10 & 0.10 & 0.09 \\
\hline
\end{tabular}

There were no significant differences in percent body weight of one-foot stance where p-values ranged from 0.32 to 0.96 over the nine regions. Like the peak pressure measurement, there were extremely high standard deviations, which comes from the variance of each participant on the plantar pressure mat. The toes, (region 1 and 2) proved to have the highest standard deviations, where some participants used their toes to grip the mat and some did not.

\section{Arch Index:}

Arch index was calculated using the frame where the area of contact was at a peak during stance and walking trials after frame calibration. Calculations were made by using the formula from Murley et al by taking $\mathrm{B} /(\mathrm{A}+\mathrm{B}+\mathrm{C})$ with the exclusion of the toes. 


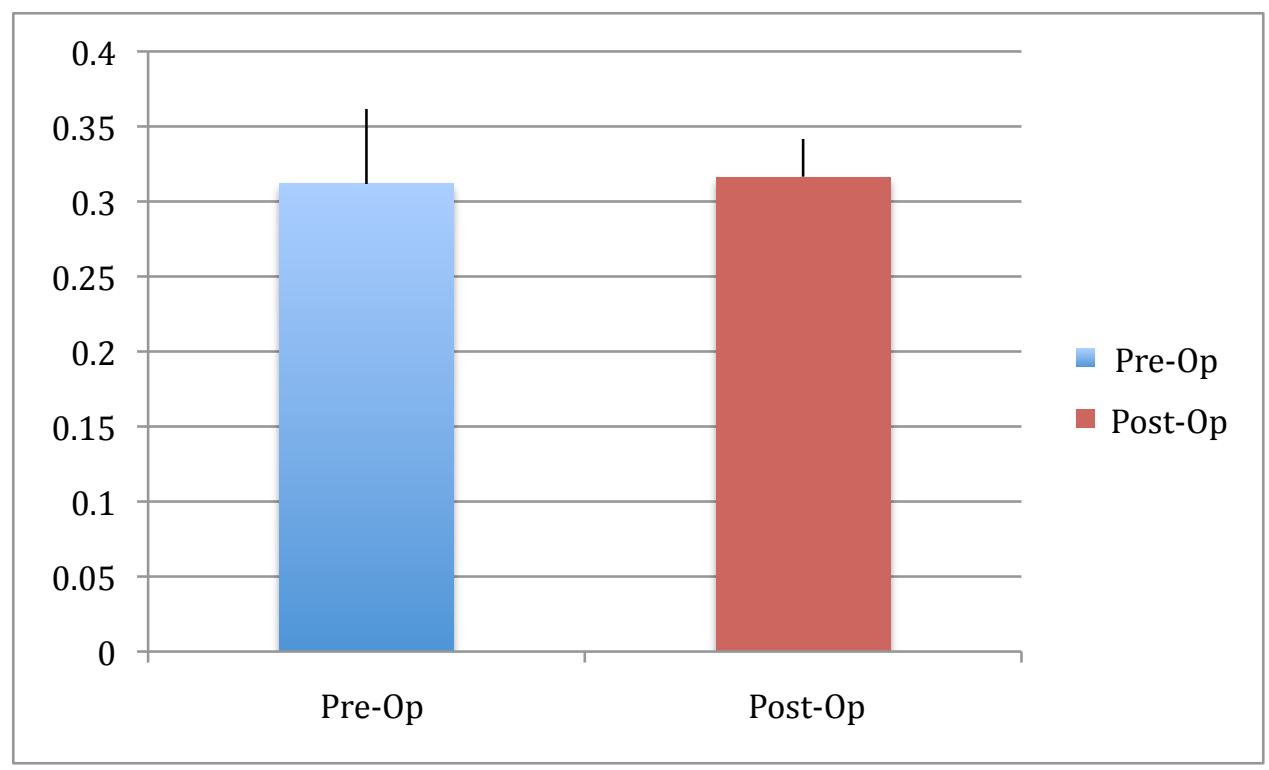

Figure 46: Arch index for two-foot stance averaged over total number of participants

Arch Index Two-Foot Stance

\begin{tabular}{|c|c|c|c|c|c|c|c|c|}
\hline Participant & 1 & 2 & 3 & 4 & 5 & 6 & Average & STDev \\
\hline Pre-Op & 0.24 & 0.37 & 0.36 & 0.30 & 0.28 & 0.32 & 0.30 & 0.04 \\
\hline Post-Op & 0.31 & - & 0.36 & 0.31 & 0.29 & 0.32 & 0.32 & 0.03 \\
\hline
\end{tabular}

An increase in arch index post-operatively would suggest a failure in surgical correction. There was no significant finding in the increase of arch index $(\mathrm{p}<0.85)$, which would suggest that this measurement might not be a good indicator of surgical success. The two-foot stance state also may have been compensated pre-operatively by favoring the unaffected leg.

One-foot stance:

It is to be noted that although there are six total pre-operative participants, that only three participants were measured for one-foot stance because the measurement was not thought of until then. Post-operatively there are five participants. One-foot stance may 
vary because of the use of the chair in front of the participant.

ONE FOOT STANCE ARCH INDEX

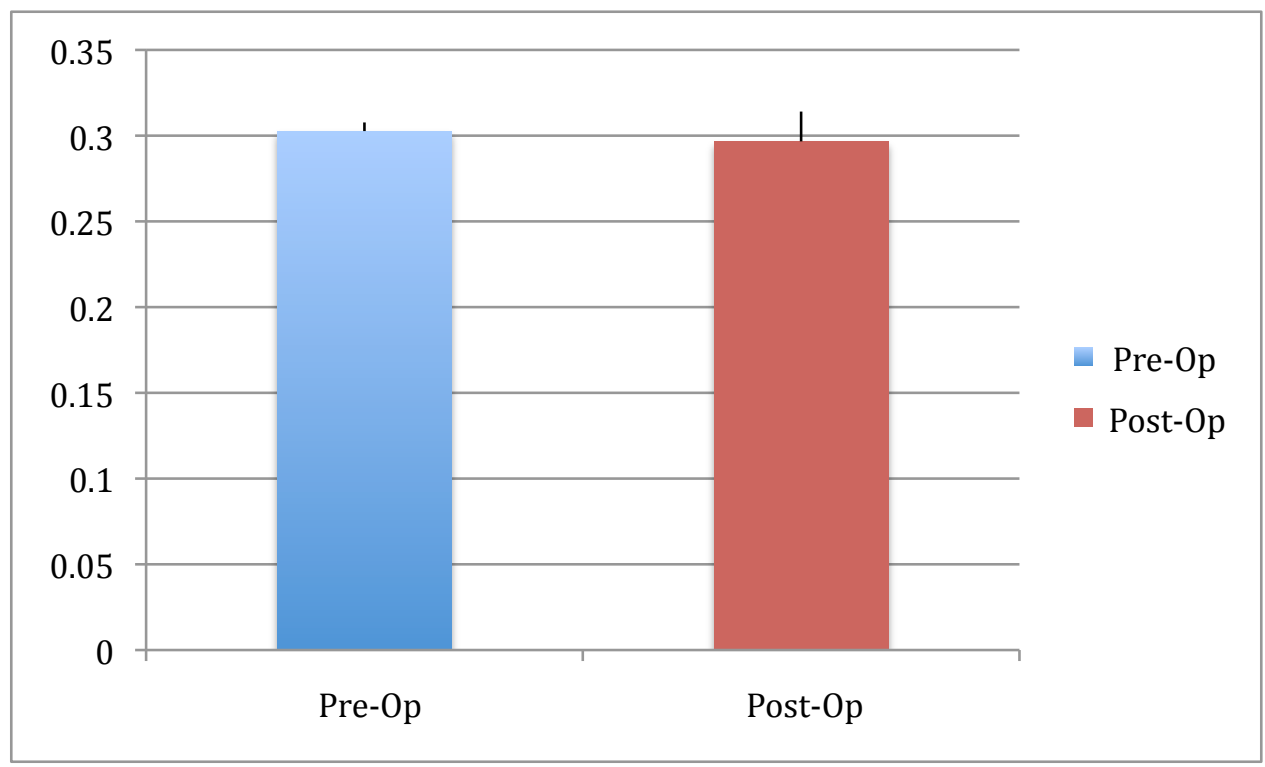

Figure 47: Arch index for one-foot stance averaged over total number of participants Arch Index One-foot Stance:

\begin{tabular}{|c|c|c|c|c|c|c|c|c|}
\hline Participant & 1 & 2 & 3 & 4 & 5 & 6 & Average & STDev \\
\hline Pre- & - & - & - & 0.30 & 0.31 & 0.30 & 0.30 & 0.01 \\
\hline Post-Op & 0.31 & - & 0.27 & 0.29 & 0.30 & 0.31 & 0.30 & 0.02 \\
\hline
\end{tabular}

Although the arch index did decrease post-operatively, it was not a significant decrease $(\mathrm{p}<0.59)$. The standard deviation of the post-operative result does encompass the pre-operative result therefore not much change occurred in one-foot stance. The postoperative number is still above the normal range for arch index. Arch index may not be a good measure for the success of the surgery for one-foot stance due to the balance variance in this measurement process. 


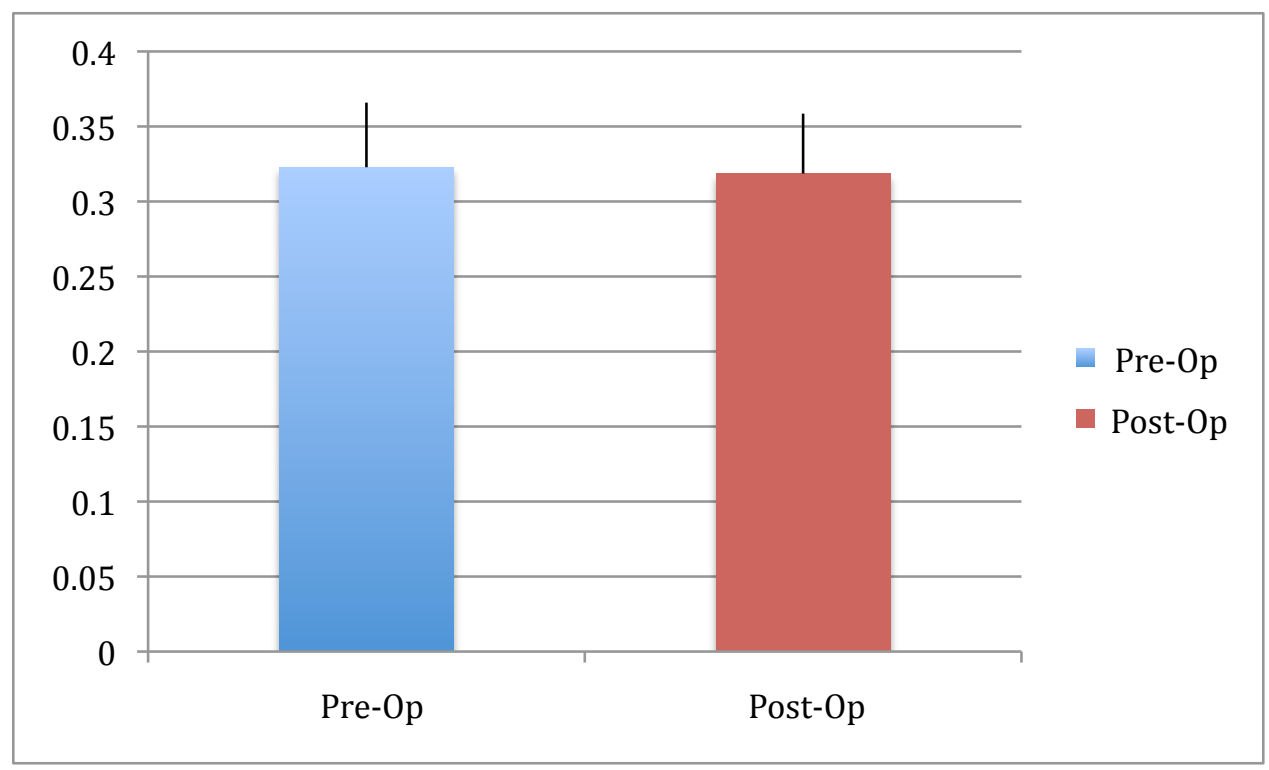

Figure 48: Peak areas over time of three walking trials and calculated arch index. Each participant's average value was averaged over the total number of participants.

Arch Index Walking:

\begin{tabular}{|c|c|c|c|c|c|c|c|c|}
\hline Participant & 1 & 2 & 3 & 4 & 5 & 6 & Average & STDev \\
\hline Pre & 0.27 & 0.35 & 0.37 & 0.30 & 0.36 & 0.29 & 0.32 & 0.04 \\
\hline Post-Op & 0.26 & - & 0.37 & 0.34 & 0.32 & 0.31 & 0.32 & 0.04 \\
\hline
\end{tabular}

In the walking trials, there was a slight decrease in arch index however nothing significant. When looking at each participant individually it is to be noted that some participants increased arch index and some did not change at all from a pre-operative to post-operative state. This may be an indicator that arch index is not a reliable measurement for determining flatfoot. 


\section{DISCUSSION \& CONCLUSIONS}

Measurement of plantar pressures provides information on how an individual's body weight is distributed to the sole of the foot (Matheis,E.A; Spratley, E.M; Hayes, C.W.; Adelaar, R.S.; Wayne,J.S., in publishing) In patients with Stage II flatfoot deformity, excessive load is placed on the medial side of the foot leading to pain and disability during gait. Surgically corrective procedures attempt to correct this imbalance and redistribute plantar pressures. This study sought to determine whether plantar pressures could be used in clinical testing to determine the success of surgical correction. Plantar pressures were measured using two methods of load distribution, in walking and stance trials, and the data between pre-operative and post-operative states compared.

Anticipated from the literature was a lateral shift in pressures through the midfoot and metatarsal regions with correction (Ledoux \& Howard, 2002) (Imhauser, Siegler, Abidi, \& Frankel, 2004) (Ellis, Yu, Johnson, Elliott, O'Malley, \& Deland, 2010). Peak pressures have been shown to exert the major influence on the dynamics of gait, however morphological differences (such as soft tissue thickness) have an influence in the variance of measurements (Cavanaugh \& Morag, 1999) (Cavanaugh P. , Morag, Boulton, 
Young, Deffner, \& Pammer, 1997) Although there have been no walking studies that include pre-operative and post-operative Stage IIb posterior tibial tendon deficient participants, there are some studies that compare walking plantar pressure between normal and flatfooted subjects and one pre-operative/post-operative case study involving the correction of flatfoot via talonavicular fusion. (Menz, Munteanu, Zammit, \& Landorf, 2010) (Ledoux \& Howard, 2002) (Fishco \& Cornwall, 2004). These studies showed a lateral shift in peak pressure and peak force during walking when going from the flatfoot state to the normal state in the forefoot, noticeably from the first metatarsophalangeal joint (1 MPJ) laterally to metatarsophalangeal joints 2-5.

From the plantar pressure analysis performed, certain measurements proved to be more successful than others in detecting differences between the pre-operative and postoperative states. This was seen in group results for walking, two-foot stance, and one-foot stance measurements when the foot was divided into nine regions. Significant results were found especially in walking segments where the lateral forefoot and midfoot increased with peak pressure and percent body weight. According to the results of our study, there were significant changes in both walking peak pressure and percent body weight, where region 5 (3-5 MPJ) showed a significant increase post-operatively $(\mathrm{p}<0.01)$ which coincides with the results of prior studies (Fishco \& Cornwall, 2004) (Ledoux \& Howard, 2002) (Menz, Munteanu, Zammit, \& Landorf, 2010). The lateral midfoot, in region 7 , only showed a significant increase $(\mathrm{p}<0.05)$ when normalizing for bodyweight over time in percent body weight measurement. Peak pressure may not have achieved a significant different pre -to post-operatively because most participants postoperatively gained weight and this measurement was not normalized for the potential 
increase in force. Although region 3 (1MPJ) and region 6 (medial midfoot) did not see a significant decrease as would be expected with flatfoot correction, both regions decreased post-operatively with percent body weight walking measurement. Unexpectedly, an increase in regions 3 and 6 was seen in the peak pressure measurement post-operatively. This again may be due to the increase in weight of each participant seen post-operatively at the one-year follow up.

Two-foot stance measurements made in our study showed trends where peak pressure decreased in region 3 and increased in regions 5,6 and 7, however not a significant amount. One-foot stance measurements for peak pressure showed an increase in regions 3,5 and 7 with a decrease in region 6 . Previous cadaveric literature exhibited decreases in the medial forefoot and midfoot (region 3, region 6) and increases in lateral forefoot and midfoot (region 5, region 7) peak pressures when compared to a normal intact state (Scott, Hendry, Iaquinto, Owen, Wayne, \& Adelaar, 2007). When compared to cadaveric data, two-foot stance saw the decrease in the medial forefoot (region 3) but did not demonstrate the decrease in medial midfoot (region 6). One-foot stance saw a decrease in the medial midfoot (region 6) but did not display a decrease in the medial forefoot (region 3), which does not match up with previous findings. Both two-foot and one-foot stance did show similar results when compared to cadaveric studies with an increase in the lateral forefoot (region 5) and the lateral midfoot (region7).

Two-foot stance measurements for percent body weight showed similar trends to peak pressure where there was a decrease in region 3 and region 6 with an increase in region 5 and region 7 . The increase in region 7 was significant $(p<0.05)$, which may be due to the normalization of participants' body weight for pre-operative and post-operative 
states. One-foot stance measurements for percent body weight showed increases in regions 3,5 , and 7 with a decrease in region 6 . Flatfoot percent body weight was drastically increased on the first metatarsophalangeal joint (region 3) and drastically decreased on the fifth metatarsophalangeal joint (region 5) when compared to a normal foot (Arangio \& Salathe, 2009). As seen post-operatively for two-foot stance, there was a decrease in percent body weight in region 3 and increase in region 5, which coincides with the literature. The increase in region 3 of one-foot stance post-operatively does not agree with the literature, but may be due to balance fluctuations in participants. While standing on one foot, participants would rely more on the forefoot and toes to "grab" the mat for balance as well as holding on to the chair in front of them, which may lead to different results than cadaveric literature.

Stance measurements in this study can be compared to findings from previous cadaveric research; however, it is to be noted that there are significant differences between in vitro and in vivo studies. For example, Imhauser et al, observed a complete lack of loading in the midfoot region in an in-vitro setting (Imhauser, Abidi, Frankel, Gavin, \& Siegler, 2002) whereas this is not true in the in-vivo setting. This may have an influence on our particular study due to differences between living tissue response and non-living tissue response. A living person may compensate for their soft tissue structures, which does not distribute the load evenly, whereas cadaveric testing applies a constant, distributed load. It is not possible to measure two-foot or one-foot stance states in a cadaveric setting, but simulating stance can be achieved by loading the soft tissue with loads that are typically found in normal stance. No two-foot stance was measured in an in vitro setting because one half of the body weight or a fixed weight could be applied 
to one foot in order to simulate two-foot stance (Scott, Hendry, Iaquinto, Owen, Wayne, \& Adelaar, 2007) (Hadfield M. , Snyder, Liacouras, Owen, Wayne, \& Adelaar, 2003) (Hadfield M. , Snyder, Liacouras, Owen, Wayne, \& Adelaar, 2005) (Arangio \& Salathe, 2009). Because living subjects usually do not distribute equal load all the time, cadaveric simulation has limitations when compared to in-vivo studies.

Measuring plantar pressures after surgical correction may be useful to predict whether patients will have discomfort in the future as lateral foot pain is a common complaint seen in flatfoot corrected individuals (Ellis, Yu, Johnson, Elliott, O'Malley, \& Deland, 2010). To assess correlation of plantar pressures of flatfoot corrected patients to their pain symptoms, a study was performed to compare the plantar pressures of patients with and without pain after surgical correction via a lateral column lengthening (Ellis, Yu, Johnson, Elliott, O'Malley, \& Deland, 2010). Post-operatively, patients with pain showed a large increase in total percent body weight of $15.7 \%$ whereas patients with no pain measured only $6.1 \%$ increase (Ellis, Yu, Johnson, Elliott, O'Malley, \& Deland, 2010). In the prior study, the medial midfoot showed that a painful percent body weight measured at $4 \%$ bodyweight; in our study two-foot stance and one-foot stance measured at $2.2 \%$ and $3.1 \%$ bodyweight and participants should not demonstrate pain. The other regions quantified in this study first metatarsophalangeal joint, fifth metatarsophalangeal joint, lateral midfoot, medial hindfoot and lateral hindfoot all had measurements above the pain threshold of participants. This would suggest that our participants may ultimately experience pain in these regions based on the increase in percent body weight,. At their one-year post-operative time point, however, most participants were happy with their surgery as suggested by the survey scores. The largest difference in percent body 
weight compared to literature was in the lateral midfoot (region 7) where our study projected that in two-foot stance and one-foot stance the percent body weights were $28.7 \%$ and $35.6 \%$ respectively. The large shift laterally suggests a surgical overcorrection that could cause potential pain in the participant's future.

Arch index has been used as a measurement to assess the degree of flatfoot of a person by splitting the foot into three zones, the forefoot, midfoot and hindfoot (Murley, Menz, \& Landorf, 2009). This measurement is based on the area of the foot on the plantar pressure mat, by dividing the area of the midfoot over the total foot area. Walking, twofoot stance and one-foot stance all showed arch index levels above the normal average value of 0.24 (Murley, Menz, \& Landorf, 2009). Although walking and one-foot stance arch index decreased slightly in group results post-operatively, the participants postoperatively would still be considered flatfooted even after surgical correction according to this measure. Two-foot stance arch index in group results increased post-operatively, which may be due to the increased loading of the afflicted foot once surgically corrected as the participant would not have to compensate on other foot. Arch index may not be a sufficient measurement for success of surgical correction since other measurements determined these particular participants to have a successful surgery.

The surveys used in our study were the SF-36 form and the FAOS survey (Appendix A) through which a correlation has been found with surgical success (Ellis, Yu, Johnson, Elliott, O'Malley, \& Deland, 2010) (Ellis, Williams, Pavolov, \& Deland, 2011). A previous survey that had been used to determine qualitative surgical success was the AOFAS (American Orthopedic Foot and Ankle Society) score in conjunction with the SF-36 form however the objective component of the scoring system may skew 
scoring validity (Madeley, Wing, Topliss, Penner, Glazebrook, \& Younger, 2012)

(Ibrahim, Beiri, Azzabi, Best, Taylor, \& Menon, 2007). The AOFAS scoring system has three components that break up the foot and ankle into regions including the anklehindfoot scale, midfoot scale, and hallux metarsophalangeal-interphalangeal scale. Because each region has a separate scoring system, it is difficult to capture the foot and ankle as a whole using this scoring method. The FAOS scoring has a broader outlook on the overall health of the foot and ankle and therefore was used in conjunction with the general health survey (SF-36).

According to the survey results of our study, the group analysis showed an increase in both SF-36 $(\mathrm{p}<0.06)$ and FAOS scoring $(\mathrm{p}<0.03)$ outcome, which would indicate a successful surgery (Madeley, Wing, Topliss, Penner, Glazebrook, \& Younger, 2012). Although one SF-36 score decreased a slight amount, the general trend showed an increase in post-operative scores. The significant increase in the foot and ankle outcome score is an extremely important finding because this survey determines the qualitative analysis of the participant about their own condition. Participants felt that their foot and ankle condition improved dramatically from pre-operative to post-operative state, which is significant in improving their daily lives.

Participants in our study show diversity among each other in many ways. Each participant had a different attitude, a different pattern of walking, a different way of standing, and a distinct opinion about their injury, however they share one common injury. Obstacles arose in repeatability and cross correlation between participants because this was a live participant study, and not cadaveric. The differences in participants encompass a wide array of patients that physicians see on a daily basis. Variance in 
walking trials was cadence, step size and head positioning, as each participant showed distinct differences. For stance trials, each participant showed different distributions of pressures due to off loading the afflicted foot. Despite measures to improve participant repeatability of walking and stance trials, variance is inevitable between subjects in this type of study.

For the walking trials, one source of variation in the measurements stemmed from difference in cadence and step size of each participant. Because each participant walked at their own selected pace, there was an inherent difference between participants.

Cadence and step size could also vary from the barefoot participant looking at the plantar pressure mat while walking toward it, which may change the gait pattern by anticipating the change in floor texture. This usually led to smaller, larger, or skipped steps prior to reaching the plantar pressure mat because the participant was intent on stepping on the mat. A change in gait cycle has an effect on repeatability of trials, therefore if the participant noticeably scuffed the plantar pressure mat or was clearly measuring the distance to the mat with their steps the trial was repeated. Each participant walked away from the researcher during the walking trial therefore, it is difficult to determine whether the participant's eyes stayed on the object in front of them or may have glanced to the pressure mat on the floor. The cadence and step size also varied within a given participant pre-operatively and post-operatively with the one-year time lapse between evaluations. This is to be expected as the participant in an injured pre-operative state may compensate for the pain connected with the afflicted foot while walking. Post-operative gait may also be changed from "normal" for the participant as they may have developed a new gait pattern with the post-operative foot. 
For two-foot stance trials, the main source of variation within each participant was the amount of force distributed over the uninjured foot in the pre-operative state. Some two-foot trials showed an uneven distribution of the center of force where the participant favored the injured foot, leaning more toward the non-afflicted foot. Post-operative twofoot stance showed more symmetric distribution of bodyweight on two feet, but some participants still had a preference for the non-afflicted foot. One-foot stance was more troublesome for repeatability as the major discrepancies between measurements were due to balance. In the pre-operative state especially, due to pain on injured foot, participants had trouble balancing on one-foot for a ten second period of time. With the aid of a chair located in front of them, they could balance if they placed one or two hands on it, however this may have skewed results as the plantar pressure distribution would increase in the forefoot and toe region. For the more skilled participants in balancing with onefoot stance, only fingers were used on the chair for stability. Depending on each participant's ability and comfort level, one-foot stance showed a large difference between participants.

Locating the metatarsophalangeal joints (MPJ) was important for creating the nine region template for each participant. Previous studies accomplished this task by pressing on each individual joint in the cadaveric specimen to locate which sensels fired on the plantar pressure apparatus (Scott, Hendry, Iaquinto, Owen, Wayne, \& Adelaar, 2007) (Hadfield M. , Snyder, Liacouras, Owen, Wayne, \& Adelaar, 2005) (Hadfield M., Snyder, Liacouras, Owen, Wayne, \& Adelaar, 2003). These studies had the advantage of the same sensels loaded where foot placement on the mat was controlled by the researcher. This approach was not feasible in an in-vivo study because the participant's 
foot would encounter the mat in different locations and orientations based on the individual. The approach followed in this study, of overlaying the plantar pressure image on the AP X-ray, was the best solution for finding the metatarsophalangeal joints. There may be a discrepancy with the overlay of the radiograph as there is a magnification that occurs with capturing a radiograph. If the X-ray image of the foot was larger, this may move the metatarsophalangeal joints slightly, however region 3 (1 MPJ) and region 5 (35 MPJ) were easily determined because of the larger area of metatarsal heads. Region 4 (2 MPJ) was difficult to define, as it was a small area comparatively to the other regions. Because of the small area and difficulty in defining the second metatarsophalangeal joint, there was a large variance in the measurements taken in region 4.

The plantar pressure apparatus has some measurement limitations that include the sensor pressure range, temperature range, durability, and performance. The sensor pressure range for Model 7101E ranges from 0 to $345 \mathrm{kPa}$ where the measurements in our study fall well between even the lowest pressure ranges. The sensor durability is also a concern, because thin polyester is used atop the plantar pressure mat as to prevent interference with the sensors. Because the participant is not standing directly on the firing sensels, there may be some error. The plantar pressure sensors are an array of areas that intersect between rows and columns creating a total image. Because there are so many sensors there may be crosstalk between sensels while loading, however this can be reduced by calibration or in the manufacturing process to isolate sensels. This particular model is considered a high-resolution mat for barefoot walking because there are 3.9 sensel $/ \mathrm{cm} 2$, which creates a better image because many sensels are firing in a small area. 
From our study, success of surgical correction of flatfoot over a diverse patient population can be quantifiably measured without invasive measures using surveys and a plantar pressure mat apparatus. For example, there was a distinct lateral shift postoperatively in walking for both the lateral forefoot and midfoot regions, which is distinct to surgical correction of flatfoot. Significant increase in the FAOS survey suggests that overall participants had better foot and ankle health post-operatively. Since it is difficult to quantify success of surgical correction non-invasively, plantar pressures accompanied with survey results could be an alternative to radiographic measures.

A clinical application of placing a plantar pressure mat in a health care office may be a way to test if surgical correction was successful by implementing walking trials and stance trials. Creating a program that measures the foot, splits it into three regions, analyzes the specific AP X-ray of the participant, and overlaying it to create the nine regions measured may be an effective tool for flatfoot surgical success as well as other foot deformities. More research and testing would need to be done in order to determine a simple protocol that could be followed clinically. 


\section{APPENDIX A:}

SHORT FORM 36 (SF-36) QUESTIONNAIRE:

http://www.anapsid.org/cnd/files/sf36.pdf

\section{SF-36 QUESTIONNAIRE}

Name:

Ref. Dr:

Date:

ID\#:

Age:

Gender: M / F

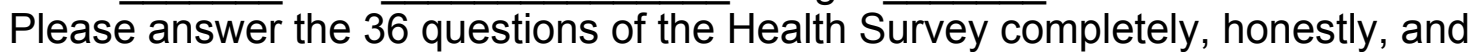
without interruptions.

1.In general, would you say your health is:

a Excellent

․ Very good

a Good

口 Fair

口 Poor

2. Compared to one year ago, how would you rate your health in general now?

$\square$ Much better now than a year ago

$\square$ Somewhat better now than a year ago

$\square$ About the same as one year ago

$\square$ Somewhat worse now than one year ago

Much worse now than one year ago

3. The following items are about activities you might do during a typical day. Does your health now limit you in these activities? If so, how much? 
a. Vigorous activities, such as running, lifting heavy objects, participating in strenuous sports.

- Yes, limited a lot.

- Yes, limited a little.

№, not limited at all.

b. Moderate activities, such as moving a table, pushing a vacuum cleaner, bowling, or playing golf?

- Yes, limited a lot.

口 Yes, limited a little.

No, not limited at all.

c. Lifting or carrying groceries.

- Yes, limited a lot.

- Yes, limited a little.

No, not limited at all.

d. Climbing several flights of stairs.

- Yes, limited a lot.

- Yes, limited a little.

- No, not limited at all.

e. Climbing one flight of stairs.

- Yes, limited a lot.

- Yes, limited a little.

- No, not limited at all.

f. Bending, kneeling or stooping.

- Yes, limited a lot.

- Yes, limited a little.

- No, not limited at all.

g. Walking more than one mile.

Yes, limited a lot.

$\checkmark$ Yes, limited a little.

$\square$ No, not limited at all.

h. Walking several blocks.

Yes, limited a lot.

$\checkmark$ Yes, limited a little.

No, not limited at all.

i. Walking one block.

$\checkmark$ Yes, limited a lot.

Yes, limited a little.

No, not limited at all. 
j. Bathing or dressing yourself.

$\square$ Yes, limited a lot.

$\square$ Yes, limited a little.

No, not limited at all.

4. During the past 4 weeks, have you had any of the following problems with your work or other regular daily activities as a result of your physical health?

a. Cut down the amount of time you spent on work or other activities?

$\square$ Yes $\square$ No

b. Accomplished less than you would like?

$\square$ Yes $\square$ No

c. Were limited in the kind of work or other activities?

$\square$ Yes $\square$ No

d. Had difficulty performing the work or other activities (for example, it took extra time)?

$\square$ Yes $\square$ No

5. During the past 4 weeks, have you had any of the following problems with your work or other regular daily activities as a result of any emotional problems (such as feeling depressed or anxious)?

a. Cut down the amount of time you spent on work or other activities?

$\square$ Yes $\square$ No

b. Accomplished less than you would like?

$\square$ Yes $\quad \square$ No

c. Didn't do work or other activities as carefully as usual?

$\square$ Yes $\square$ No

6. During the past 4 weeks, to what extent has your physical health or emotional problems interfered with your normal social activities with family, friends, neighbors, or groups?

$\square$ Not at all $\square$ Slightly $\square$ Moderately $\square$ Quite a bit $\square$ Extremely

7. How much bodily pain have you had during the past 4 weeks?

$\square$ Not at all $\square$ Slightly $\square$ Moderately $\square$ Quite a bit $\square$ Extremely 
8. During the past 4 weeks, how much did pain interfere with your normal work (including both work outside the home and housework)?

$\square$ Not at all $\square$ Slightly $\square$ Moderately $\square$ Quite a bit $\square$ Extremely

9. These questions are about how you feel and how things have been with you during the past 4 weeks. For each question, please give the one answer that comes closest to the way you have been feeling. How much of the time during the past 4 weeks.

a. did you feel full of pep?

All of the time

Most of the time

$\square$ A good bit of the time

$\square$ Some of the time

A little of the time

None of the time

b. have you been a very nervous person?

All of the time

Most of the time

A good bit of the time

$\square$ Some of the time

A little of the time

None of the time

c. have you felt so down in the dumps nothing could cheer you up?

All of the time

Most of the time

A good bit of the time

$\square$ Some of the time

$\square$ A little of the time

None of the time

d. have you felt calm and peaceful?

All of the time

Most of the time

$\square$ A good bit of the time

Some of the time

A little of the time

None of the time

e. did you have a lot of energy?

All of the time

Most of the time

A good bit of the time

Some of the time 
A little of the time

None of the time

f. have you felt downhearted and blue?

All of the time

Most of the time

A good bit of the time

Some of the time

A little of the time

None of the time

g. did you feel worn out?

$\square$ All of the time

Most of the time

A good bit of the time

Some of the time

A little of the time

None of the time

h. have you been a happy person?

All of the time

Most of the time

A good bit of the time

$\square$ Some of the time

A little of the time

None of the time

i. did you feel tired?

All of the time

Most of the time

A good bit of the time

$\square$ Some of the time

A little of the time

None of the time

10. During the past 4 weeks, how much of the time has your physical health or emotional problems interfered with your social activities (like visiting friends, relatives, etc.)?

All of the time

$\square$ Most of the time

$\square$ Some of the time

A little of the time

$\square$ None of the time

11. How TRUE or FALSE is each of the following statements for you? 
a. I seem to get sick a little easier than other people

$\square$ Definitely true

Mostly true

Don't know

Mostly false

Definitely false

b. I am as healthy as anybody I know

$\square$ Definitely true

Mostly true

Don't know

Mostly false

$\square$ Definitely false

FOOT AND ANKLE OUTCOME SURVEY (FAOS):

http://www.koos.nu/FAOSEng.pdf

Foot and Ankle Outcome Score (FAOS), English version LK1.0

FAOS FOOT \& ANKLE SURVEY

Todays date: Date of birth:

Name:

INSTRUCTIONS: This survey asks for your view about your foot/ankle. This information will help us keep track of how you feel about your foot/ankle and how well you are able to do your usual activities. Answer every question by ticking the appropriate box, only one box for each question. If you are unsure about how to answer a question, please give the best answer you can.

\section{Symptoms}

These questions should be answered thinking of your foot/ankle symptoms during the last week.

S1. Do you have swelling in your foot/ankle?

Never Rarely Sometimes Often Always

S2. Do you feel grinding, hear clicking or any other type of noise when your foot/ankle moves?

Never

Rarely Sometimes

Often

Always

S3. Does your foot/ankle catch or hang up when moving?

Never Rarely Sometimes Often

Always 
S4. Can you straighten your foot/ankle fully?

Never Rarely Sometimes Often Always

S5. Can you bend your foot/ankle fully?

Never Rarely Sometimes Often Always

\section{Stiffness}

The following questions concern the amount of joint stiffness you have experienced during the last week in your foot/ankle. Stiffness is a sensation of restriction or slowness in the ease with which you move your joints.

S6. How severe is your foot/ankle stiffness after first wakening in the morning?

None

Mild

Moderate

Severe

Extreme

S7. How severe is your foot/ankle stiffness after sitting, lying or resting later in the day?

None Mild Moderate Severe Extreme

Pain

P1. How often do you experience foot/ankle pain?

Never

Monthly

Weekly

Daily

Always

What amount of foot/ankle pain have you experienced the last week during the following activities?

P2. Twisting/pivoting on your foot/ankle

None Mild Moderate

P3. Straightening foot/ankle fully

None Mild Moderate

Severe $\quad$ Extreme

Severe

Extreme

P4. Bending foot/ankle fully

None

Mild

Moderate

Severe

Extreme

P5. Walking on flat surface

None

Mild

Moderate

Severe

Extreme

P6. Going up or down stairs

None

Mild

Moderate

Severe

Extreme

P7. At night while in bed

None

Mild

Moderate

Severe

Extreme

P8. Sitting or lying

None $\quad$ Mild

P9. Standing upright

None Mild

Moderate

Severe

Extreme 


\section{Function, daily living}

The following questions concern your physical function. By this we mean your ability to move around and to look after yourself. For each of the following activities please indicate the degree of difficulty you have experienced in the last week due to your foot/ankle.

A1. Descending stairs

None Mild

Moderate Severe Extreme

A2. Ascending stairs

None Mild

Moderate Severe Extreme

A3. Rising from sitting

None Mild Moderate Severe Extreme

A4. Standing

None

Mild

Moderate

Severe

Extreme

A5. Bending to floor/pick up an object

None Mild Moderate

Severe Extreme

A6. Walking on flat surface

None Mild Moderate Severe Extreme

A7. Getting in/out of car

None Mild Moderate Severe Extreme

A8. Going shopping

None Mild Moderate Severe Extreme

A9. Putting on socks/stockings

None Mild Moderate Severe Extreme

A10. Rising from bed

None Mild Moderate Severe Extreme

A11. Taking off socks/stockings

None Mild Moderate Severe Extreme

A12. Lying in bed (turning over, maintaining foot/ankle position)

None Mild Moderate Severe Extreme

A13. Getting in/out of bath

None Mild Moderate Severe Extreme 
A14. Sitting

None Mild $\quad$ Moderate Severe Extreme

A15. Getting on/off toilet

None Mild Moderate Severe Extreme

A16. Heavy domestic duties (moving heavy boxes, scrubbing floors, etc)

None Mild Moderate Severe Extreme

A17. Light domestic duties (cooking, dusting, etc)

None Mild Moderate Severe Extreme

\section{Function, sports and recreational activities}

The following questions concern your physical function when being active on a higher level. The questions should be answered thinking of what degree of difficulty you have experienced during the last week due to your foot/ankle.

SP1. Squatting

None

Mild

Moderate

Severe

Extreme

SP2. Running

None

Mild

Moderate

Severe

Extreme

SP3. Jumping

None

Mild

Moderate

Severe

Extreme

SP4. Twisting/pivoting on your injured foot/ankle

None Mild Moderate Severe Extreme

SP5. Kneeling

None Mild $\quad$ Moderate Severe Extreme

\section{Quality of Life}

Q1. How often are you aware of your foot/ankle problem?

Never Monthly Weekly Daily Constantly

Q2. Have you modified your life style to avoid potentially damaging activities to your foot/ankle?

$\begin{array}{llll}\text { Not at all Mildly } & \text { Moderately } & \text { Severely } & \text { Totally }\end{array}$

Q3. How much are you troubled with lack of confidence in your foot/ankle?

Not at all Mildly Moderately Severely Extremely

Q4. In general, how much difficulty do you have with your foot/ankle?
None
Mild
Moderate
Severe
Extreme 


\section{APPENDIX B}

PARTICIPANT 2 (Left foot afflicted)

Participant 2 was first brought in on 9/22/2010 for plantar pressure analysis, radiographs, and motion analysis. Radiographs were taken first at third floor Gateway of MCV the participant traveled over to Broad St. for motion analysis then plantar pressure protocol.

Participant 2 did not return for a post-operative follow-up.

\section{Pre-Op Comments:}

Participant 2 showed a genuine interest in this study, as she was very curious about how the surgery was going to work. She had a positive outlook about her situation and was looking forward to seeing the results of her plantar pressures as well as radiographs and MRI. She completed each survey commenting that she "was going to be able to do all of these activities after surgery". Standing on the pressure mat was good for two-foot, but she had a bit of a challenge with one-foot stance, occasionally bobbing from side to 
side. Although she struggled a little bit to move around she was determined to make each walking trial correct. There were about 15 total walking trials for the plantar pressure analysis where three trials for each foot were recorded.

\section{Post-Op Comments:}

It is to be noted that this participant did not return for post-op analysis, but is included in the pre-operative general results.

\section{Surveys:}

The SF-36 is a determinate of overall health and quality of life on a percent based scale where if the participant is closer to $100 \%$ then the general health is good.

\section{SF-36}

Pre-Op: 51

The FAOS (foot and ankle outcome score) is a determinate of the health of the foot and ankle as well as scaling the level of activities of daily living and overall health due to foot and ankle problems. This scale is out of 500, where the higher the total score, the less affected the foot and ankle issue is to the participant.

\section{FAOS}

Pre-Op: 190

Because the participant did not return for post-operative evaluations, no comparisons can be made between a pre-operative and post-operative state, but it can be seen that the participant clearly functioned at a lower level on both surveys. 


\section{Plantar pressure images:}

Participant 2's individual plantar pressure images are shown at peak pressure over time (Figure A.1). These images show the maximum amount of firing on each sensel over total time on the plantar pressure mat. For the stance measurement the largest group of frame captures were used where the participant moved very little. The middle frame of the group was chosen as the most steady and used for calibration. Walking images shown are the total time it took for the participant to go from heel strike to toe off. 


\section{PARTICIPANT 2}

$\underline{\mathrm{kPa}}$

Two -Foot Stance

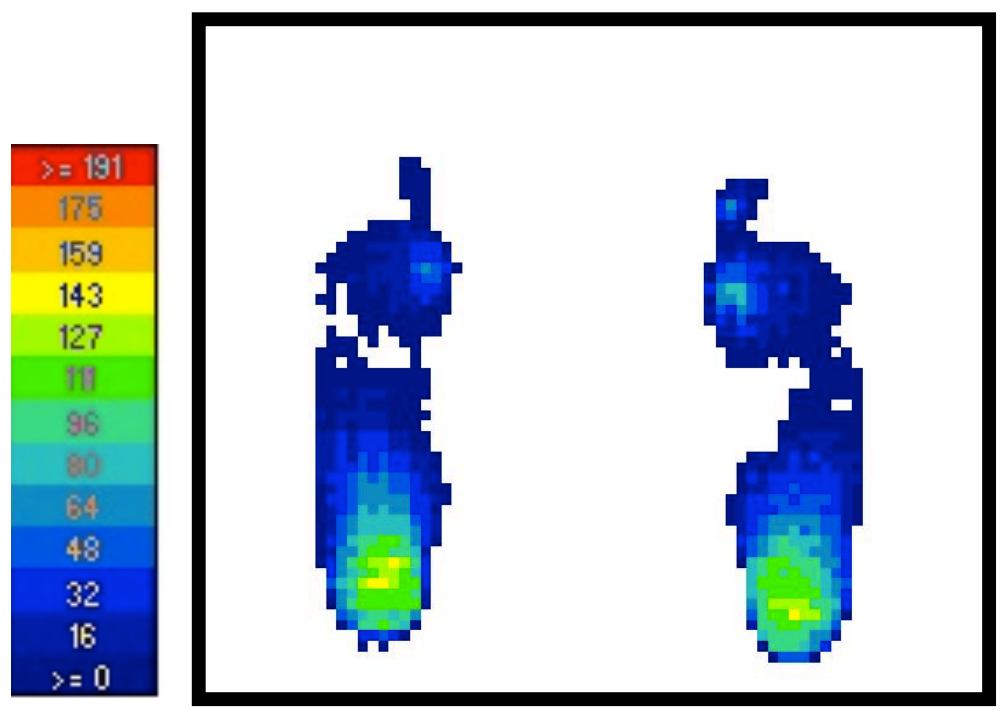

Walking
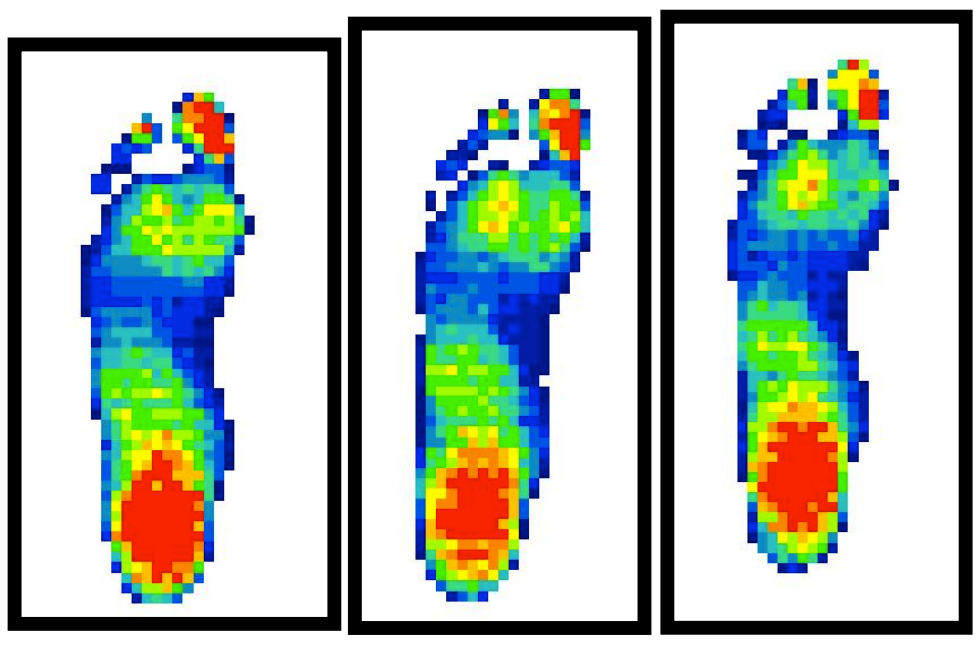

Trial 1

$\underline{\text { Trial } 2}$

Trial 3

Figure A.1: Pre-0p Plantar pressure images for Participant 2 


\section{Walking:}

Three trials of each foot for walking analysis was performed and compared to preop analysis. Calibration of the plantar pressure mat was done by frame calibration where for each walking trial the middle frame of midstance was used.

\section{$\underline{\text { Peak Pressure: }}$}

Peak pressure is measured by taking the peak pressure in each region over the total time of the walking cycle. Each peak represents a distinct instant of time in the gait cycle for each region. This does not represent only one event in time, but nine separate events.

\section{WALKING PEAK PRESSURE}

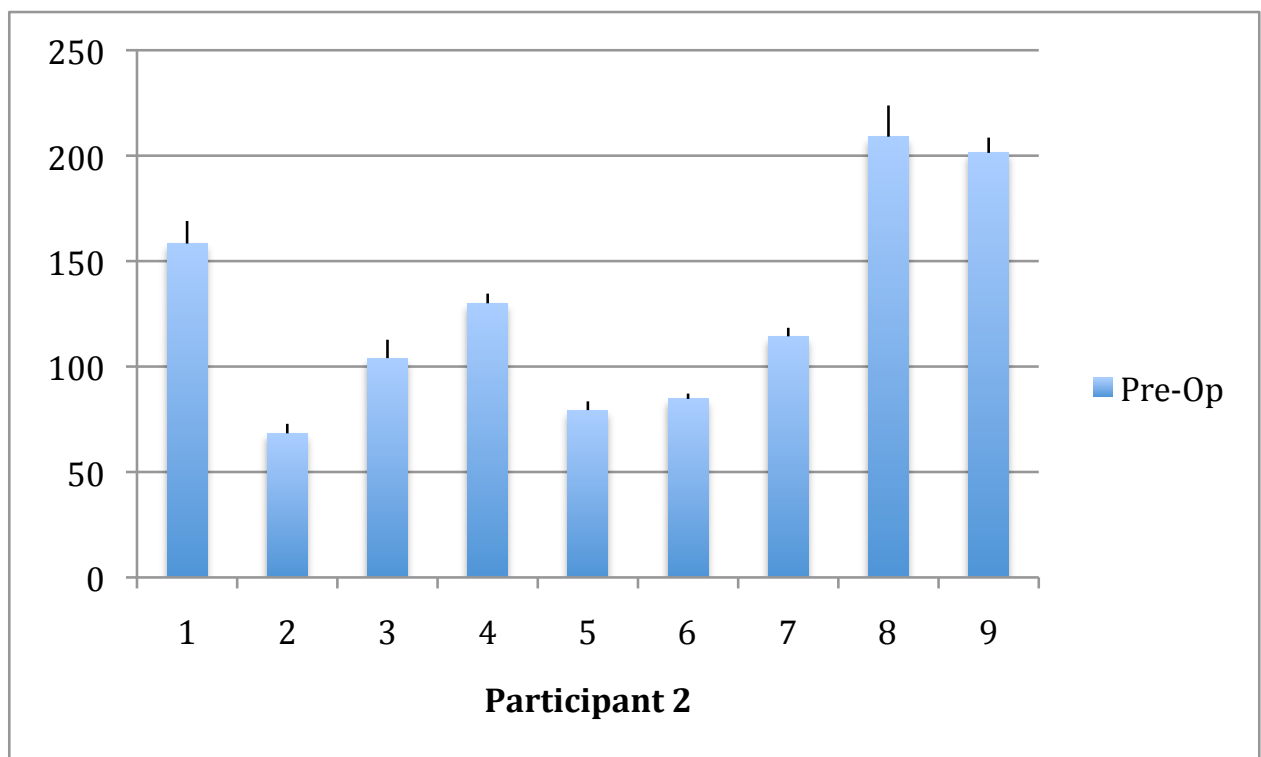

Figure A.2: Peak pressure in each of the nine regions defined over the entire walking cycle averaged over three trials of the afflicted foot for Participant 2. 
Pre-Op Peak Pressures (kPa):

\begin{tabular}{|l|r|r|r|r|r|r|r|r|r|}
\hline Regions & 1 & 2 & 3 & 4 & 5 & 6 & 7 & 8 & 9 \\
\hline Average & 158 & 68 & 104 & 130 & 79 & 85 & 114 & 209 & 201 \\
\hline $\begin{array}{l}\text { Standard } \\
\text { Deviation }\end{array}$ & 11 & 5 & 9 & 5 & 4 & 3 & 4 & 15 & 7 \\
\hline
\end{tabular}

\section{Percent Body Weight:}

The measurement of percent body weight was taken in each region by the maximum force in pounds in that region on the afflicted foot and dividing it by the total body weight of the participant. This was done so that the measurements can be compared across time if the bodyweight of the participant changed. The participant was weighed pre-operatively (194 lbs).

\section{WALKING PERCENT BODY WEIGHT}

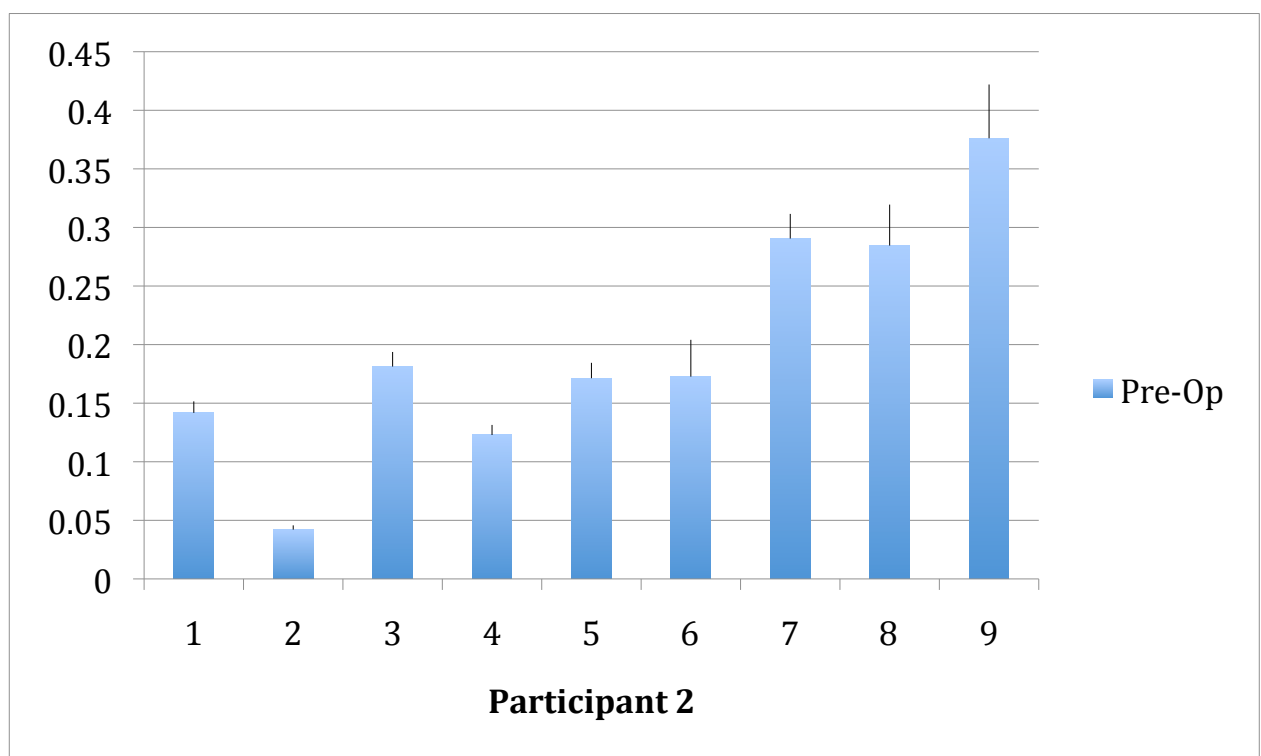

Figure A.3: Maximum percent body weight over time of the walking trial for the average of three trials of the afflicted foot for Participant 2 
Pre-Op Percent Body Weight

\begin{tabular}{|l|r|r|r|r|r|r|r|r|r|}
\hline Regions & 1 & 2 & 3 & 4 & 5 & 6 & 7 & 8 & 9 \\
\hline Average & 0.14 & 0.04 & 0.18 & 0.12 & 0.17 & 0.17 & 0.29 & 0.28 & 0.38 \\
\hline $\begin{array}{l}\text { Standard } \\
\text { Deviation }\end{array}$ & 0.01 & 0.00 & 0.01 & 0.01 & 0.01 & 0.03 & 0.02 & 0.03 & 0.05 \\
\hline
\end{tabular}

Since there are no post-operative results to compare, it can be noted that there is high pressure and percent body weight in region 6 , which is to be expected with someone with a fallen arch.

\section{Stance:}

Trials were taken with the aid of a chair for balance (if needed) for both two-foot stance as well as one-foot stance. For one-foot stance, both afflicted foot and un-afflicted foot were collected, however only the afflicted foot results are presented. Frame calibration was used to calibrate the trials by taking the middle frame of the collected response (10 seconds) for both two-foot and one-foot stance. 


\section{Two-Foot Stance:}

TWO-FOOT STANCE PEAK PRESSURE

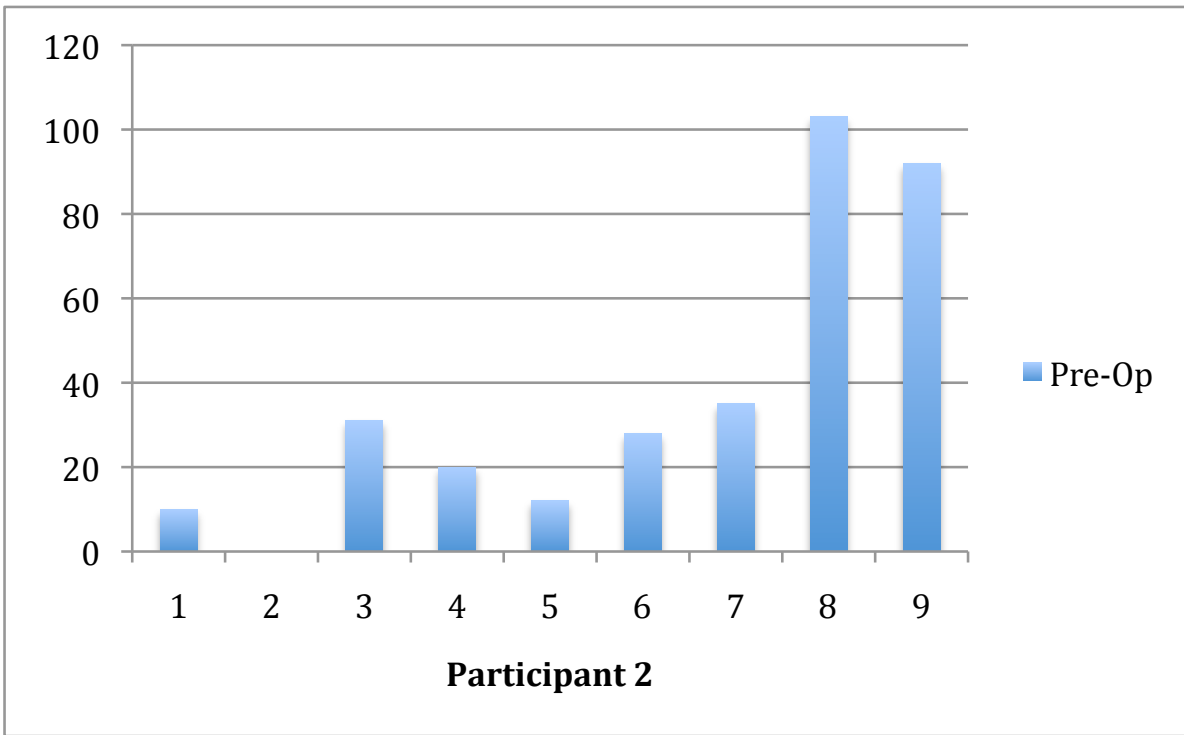

Figure A.4: Peak pressure measurements in regions over total stance time with twofoot stance for the afflicted foot

Pre-Op Pressure $(\mathrm{kPa})$ :

$\begin{array}{rrrrrrrrrr}\text { Regions } & 1 & 2 & 3 & 4 & 5 & 6 & 7 & 8 & 9 \\ & 10 & 0 & 31 & 20 & 12 & 28 & 35 & 103 & 92\end{array}$

\section{TWO-FOOT STANCE PERCENT BODY WEIGHT}

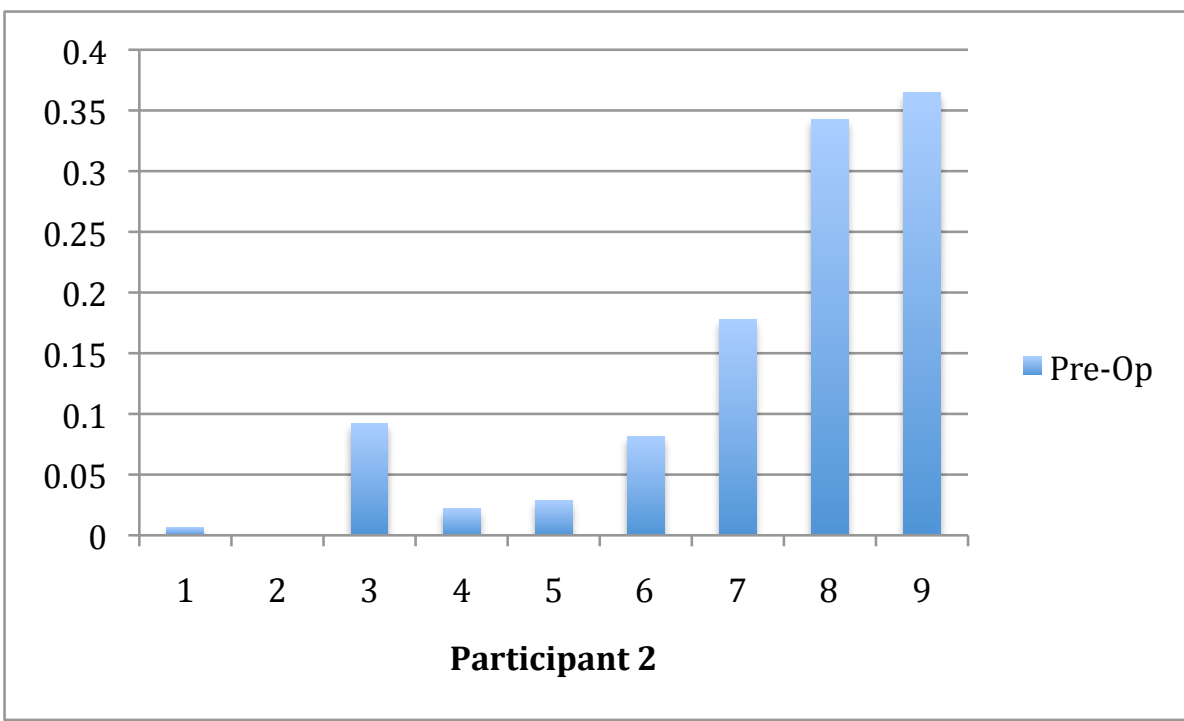

Figure A.5: Peak pressure measurements in regions over total stance time with two-foot stance for the afflicted foot 
Pre-Op Percent Body Weight:

\begin{tabular}{|l|r|r|r|r|r|r|r|r|r|}
\hline Regions & 1 & 2 & 3 & 4 & 5 & 6 & 7 & 8 & 9 \\
\hline & 0.01 & 0.00 & 0.09 & 0.02 & 0.03 & 0.08 & 0.18 & 0.34 & 0.37 \\
\hline
\end{tabular}

Increases in regions 3 and 6 are indicative of a flatfoot condition with a shift toward the medial aspect of the foot in two-foot stance for both peak pressure and percent body weight.

\section{Arch Index:}

Arch index was calculated using the frame where the area of contact was at a peak during stance and walking trials after frame calibration. Calculations were made by using the formula from Murley et al by taking $B /(A+B+C)$ with the exclusion of the toes.

\section{TWO FOOT STANCE ARCH INDEX}

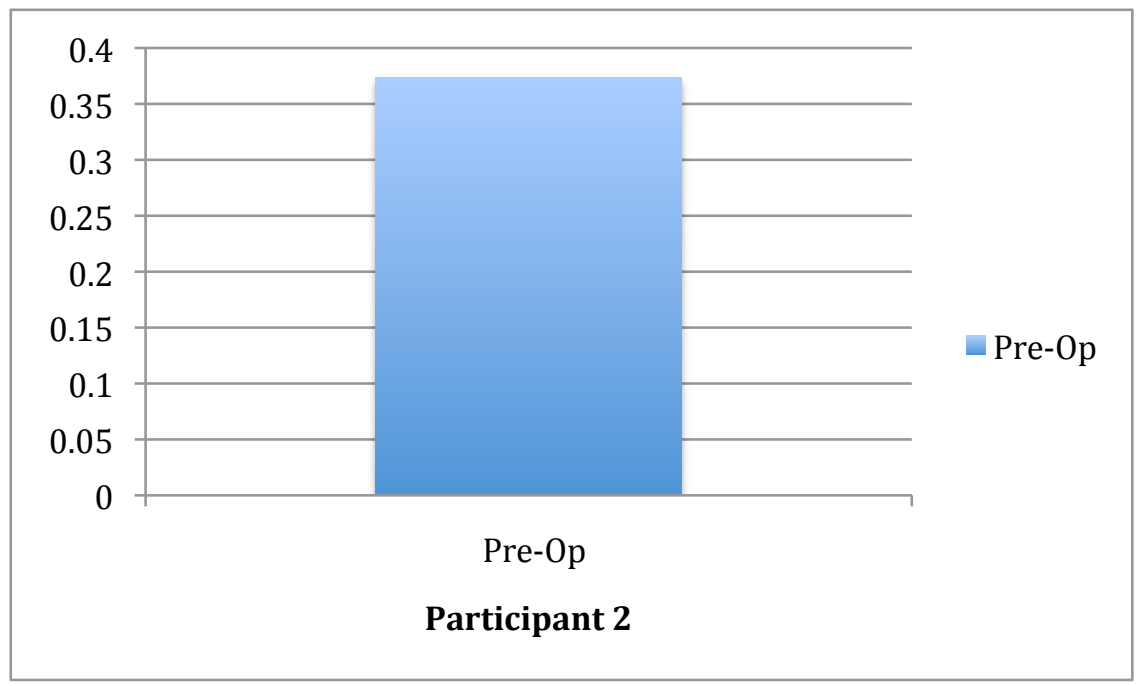

Figure A.6: Arch index for stance of total peak area over time for two-foot stance Pre-Op Two-Foot Arch Index: 0.37 


\section{WALKING ARCH INDEX}

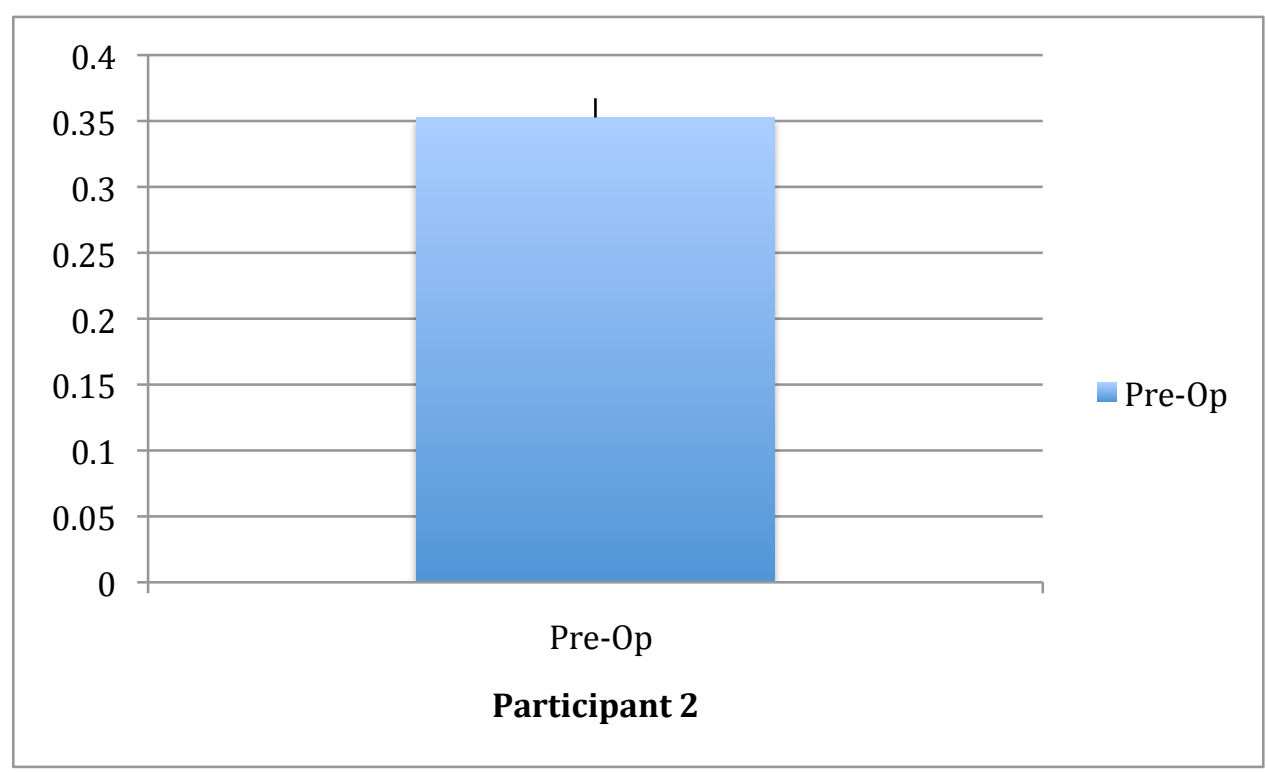

Figure A.7: Arch index measurements of the peak areas over time of three walking trials.

Pre-Op Arch Index

\begin{tabular}{|r|r|r|r|r|r|r|}
\hline Forefoot & Midfoot & Hindfoot & Total & $\begin{array}{l}\text { Arch } \\
\text { Index }\end{array}$ & $\begin{array}{l}\text { Average } \\
\text { AI }\end{array}$ & STD Dev \\
\hline 51 & 45 & 32 & 128 & 0.35 & & \\
\hline 51 & 46 & 29 & 126 & 0.37 & & \\
\hline 51 & 42 & 30 & 123 & 0.34 & 0.35 & 0.01 \\
\hline
\end{tabular}

PARTICIPANT 3 (Right foot afflicted)

Participant 3 was first brought in on 10/06/2010 for plantar pressure analysis, radiographs, and motion analysis. Radiographs were taken first at third floor Gateway of MCV then participant traveled over to Broad St. to do motion analysis then plantar pressure protocol. 
Participant 3 was brought back on 1/17/2012 for plantar pressure analysis and radiographs. No motion analysis was done. Plantar pressures were done in the Ambulatory Care Center in a spare protocol room. Radiographs were taken at third floor Gateway of MCV.

\section{Pre-Op Comments:}

The participant arrived with a boot on her foot and a cheery attitude. There were extra trials done of plantar pressure as well as standing on one leg and two legs. The extra trials may have exacerbated her foot more. Her gait pattern was clean. I got one foot on each walking trial. The walking was done in the gait analysis lab after about 25 trials of walking which also may have affected the status of her foot.

Three trials of each walking analysis of each foot were performed, and when compared to my normal trials, the participant showed significant flatfoot. Out of the particpants seen she had a noticeably visible flatfoot according to plantar pressure readings where the midfoot had clearly collapsed.

\section{Post- Op Comments:}

After surgical correction via a medial calcaneal osteotomy (MCO) and tendon transfer, the participant returned for testing. The participant arrived with a cheery attitude and was ready to walk. She said she felt much better walking and even worked out the day before. Due to noise of the plantar pressure mat during the triggered walking trials there were many trials of this participant. Also with the practice trials and subsequent trials after the practice trials, the participant had a difficult time placing the correct foot on the mat. There were approximately 20 trials total. She did not need to sit down at all 
and felt great after. No motion analysis was done. Both two-foot and one-foot stance was captured and analyzed.

\section{Surveys:}

The SF-36 is a determinate of overall health and quality of life on a percent based scale where if the participant is closer to $100 \%$ then the general health is good.

\section{$\underline{\mathrm{SF}-36}$}

Pre-Op: 21

Post-Op: 84

It is to be noted that the participant did not answer the last four questions of this survey during the pre-operative stage. The score was tabulated based on the answered questions and the other questions were then omitted. There is a dramatic increase in general health function post-operatively. This participant saw a more than $60 \%$ increase in general health!

The FAOS (foot and ankle outcome score) is a determinate of the health of the foot and ankle as well as scaling the level of activities of daily living and overall health due to foot and ankle problems. This scale is out of 500, where the higher the total score, the less affected the foot and ankle issue is to the participant.

\section{FAOS}

Pre-Op: 102

Post-Op: 413

There was an over 300-point increase on her foot an ankle functioning after surgery. This is over a $60 \%$ increase in function! According to the surveys this participant was extremely happy with how her foot was functioning post surgery. 


\section{Plantar pressure images:}

Participant 3's individual plantar pressure images are shown at peak pressure over time (Figures A.8 and A.9). These images show the maximum amount of firing on each sensel over total time on the plantar pressure mat. For the stance measurement the largest group of frame captures were used where the participant moved very little. The middle frame of the group was chosen as the most steady and used for calibration. Walking images shown are the total time it took for the participant to go from heel strike to toe off.

\section{PRE-OP PLANTAR PRESSURE IMAGES}

\section{$\underline{\text { PARTICIPANT } 3}$}

\section{Two -Foot Stance}

$\underline{\mathrm{kPa}}$








Trial 1

$\underline{\text { Trial } 2}$

$\underline{\text { Trial } 3}$

Figure A.8: Pre-Op Plantar pressure images for Participant 3

POST-OP PLANTAR PRESSURE IMAGES

$\underline{\text { PARTICPANT } 3}$ Two-Foot Stance $\quad$ One-Foot Stance






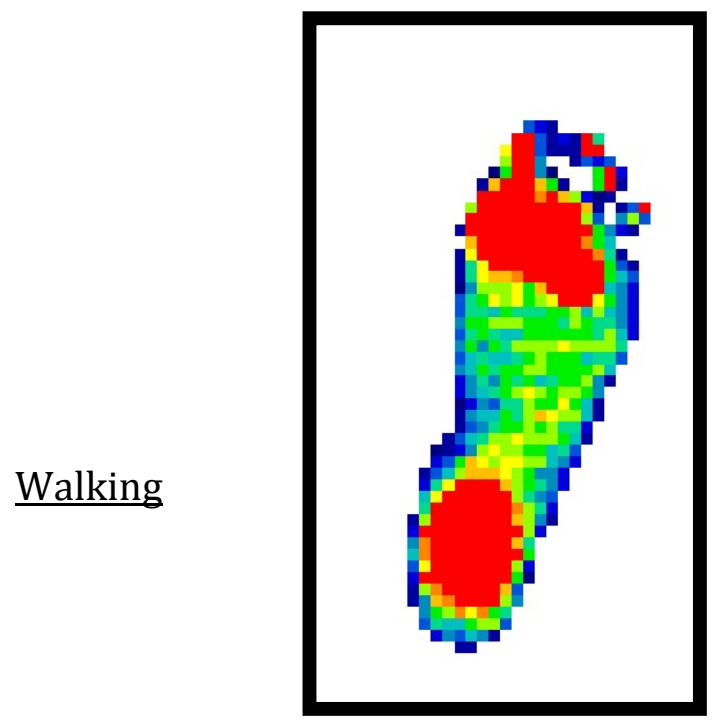

$\underline{\text { Trial } 1}$

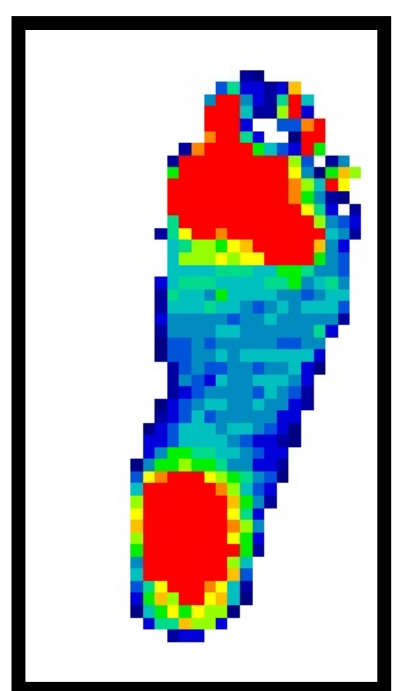

Trial 2

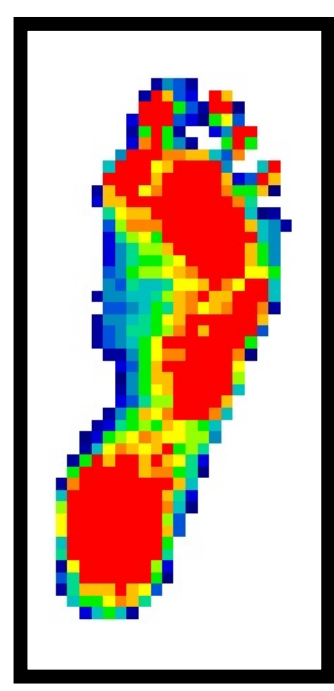

$\underline{\text { Trial } 3}$

Figure A.9: Post-Op Plantar Pressure images for Participant 3

\section{Walking:}

Three trials of each foot for walking analysis was performed and compared to preop analysis. Calibration of the plantar pressure mat was done by frame calibration where for each walking trial the middle frame of midstance was used.

\section{Peak Pressure:}

Peak pressure is measured by taking the peak pressure in each region over the total time of the walking cycle. Each peak represents a distinct instant of time in the gait cycle for each region. This does not represent only one event in time, but nine separate events. 
WALKING PEAK PRESSURE

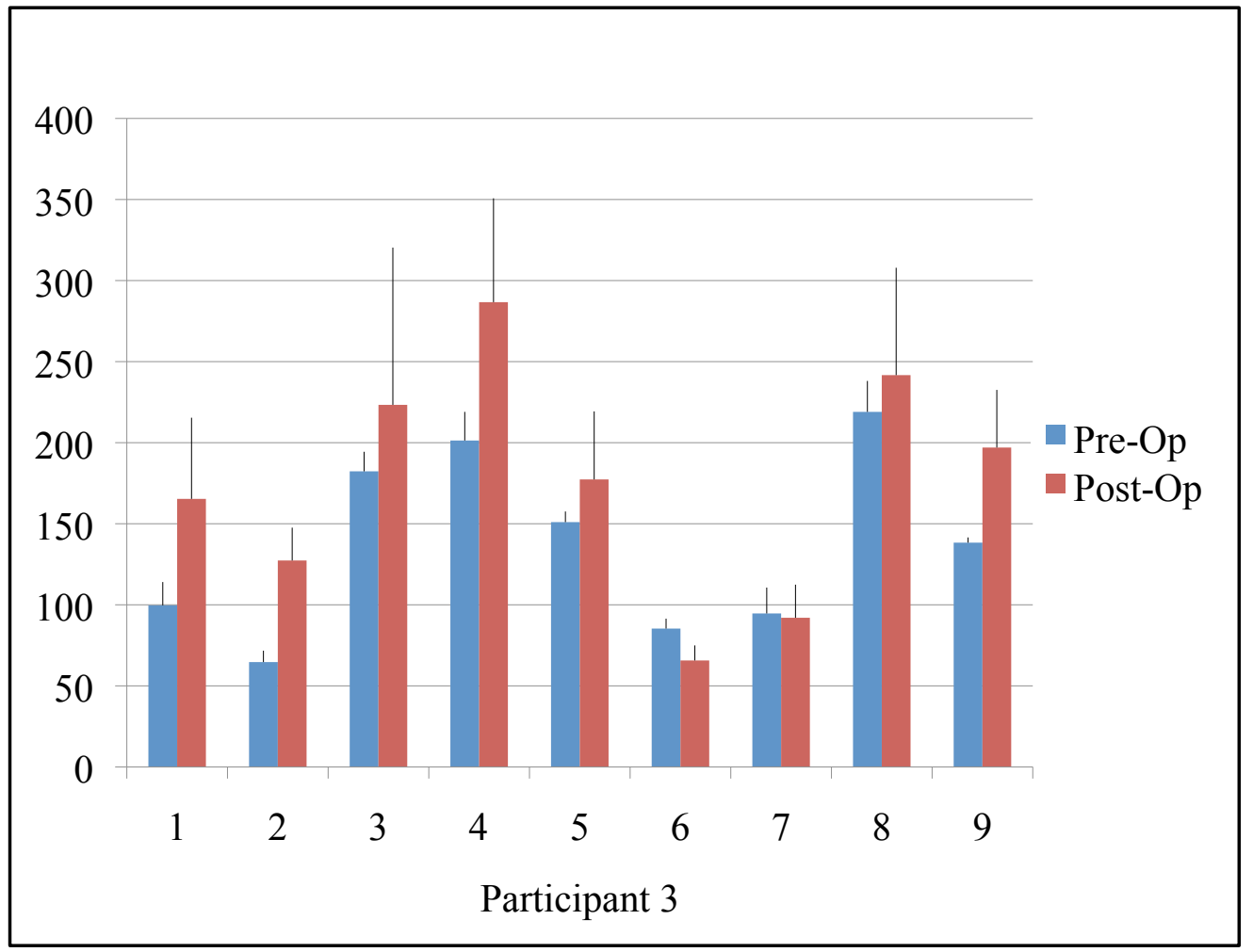

Figure A.10: Peak pressure in each of the nine regions defined over the entire walking cycle averaged over three trials of the afflicted foot for Participant 3.

Pre-Op Peak Pressures (kPa):

\begin{tabular}{|l|r|r|r|r|r|r|r|r|r|}
\hline Regions & 1 & 2 & 3 & 4 & 5 & 6 & 7 & 8 & 9 \\
\hline Average & 100 & 65 & 182 & 201 & 151 & 85 & 95 & 219 & 138 \\
\hline $\begin{array}{l}\text { Standard } \\
\text { Deviation }\end{array}$ & 14 & 7 & 12 & 18 & 7 & 6 & 16 & 19 & 3 \\
\hline
\end{tabular}

Post-Op Peak Pressures (kPa):

\begin{tabular}{|l|r|r|r|r|r|r|r|r|r|}
\hline Regions & 1 & 2 & 3 & 4 & 5 & 6 & 7 & 8 & 9 \\
\hline Average & 165 & 127 & 223 & 287 & 177 & 66 & 92 & 242 & 197 \\
\hline $\begin{array}{l}\text { Standard } \\
\text { Deviation }\end{array}$ & 50 & 20 & 97 & 64 & 42 & 9 & 20 & 66 & 36 \\
\hline
\end{tabular}

\section{Percent Body Weight:}

The measurement of percent body weight was taken in each region by the maximum force in pounds in that region on the afflicted foot and dividing it by the total body weight of the participant. This was done so that the measurements can be compared 
across time if the bodyweight of the participant changed. The participant was weighed both pre-operatively (209.2 lbs) and post-operatively (208.8 lbs).



Figure A.11 Maximum percent body weight over time of the walking trial for the average of three trials on afflicted foot for Participant 3.

Pre-Op Percent Body Weight:

\begin{tabular}{|l|r|r|r|r|r|r|r|r|r|}
\hline Regions & 1 & 2 & 3 & 4 & 5 & 6 & 7 & 8 & 9 \\
\hline Average & 0.06 & 0.04 & 0.40 & 0.22 & 0.31 & 0.16 & 0.24 & 0.31 & 0.21 \\
\hline $\begin{array}{l}\text { Standard } \\
\text { Deviation }\end{array}$ & 0.01 & 0.00 & 0.01 & 0.01 & 0.03 & 0.02 & 0.05 & 0.01 & 0.02 \\
\hline
\end{tabular}

Post-Op Percent Body Weight:

\begin{tabular}{|l|r|r|r|r|r|r|r|r|r|}
\hline Regions & 1 & 2 & 3 & 4 & 5 & 6 & 7 & 8 & 9 \\
\hline Average & 0.09 & 0.07 & 0.36 & 0.19 & 0.35 & 0.11 & 0.24 & 0.31 & 0.32 \\
\hline $\begin{array}{l}\text { Standard } \\
\text { Deviation }\end{array}$ & 0.03 & 0.02 & 0.18 & 0.03 & 0.06 & 0.05 & 0.12 & 0.09 & 0.07 \\
\hline
\end{tabular}




\section{Stance:}

Trials were taken with the aid of a chair for balance (if needed) for both two-foot stance as well as one-foot stance. For one-foot stance, both afflicted foot and un-afflicted foot were collected, however only the afflicted foot results are presented. Frame calibration was used to calibrate the trials by taking the middle frame of the collected response (10 seconds) for both two-foot and one-foot stance.

\section{Two-Foot Stance:}

TWO-FOOT STANCE PEAK PRESSURE

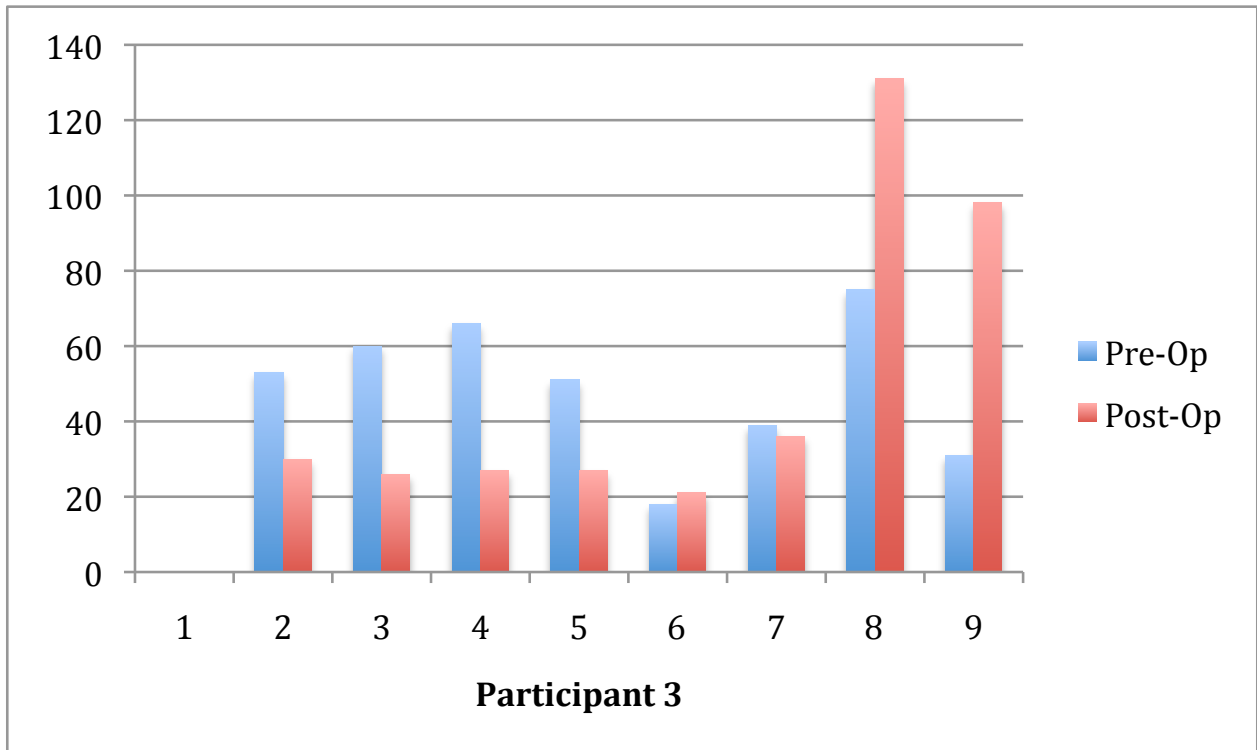

Figure A.12: Peak pressure measurements in regions over total stance time with two-foot stance for the afflicted foot

Pre-Op Peak Pressure $(\mathrm{kPa})$ :

\begin{tabular}{|l|r|r|r|r|r|r|r|r|r|}
\hline Regions & 1 & 2 & 3 & 4 & 5 & 6 & 7 & 8 & 9 \\
\hline & 0 & 53 & 60 & 66 & 51 & 18 & 39 & 75 & 31 \\
\hline
\end{tabular}

Post-Op Peak Pressure $(\mathrm{kPa})$ :

\begin{tabular}{|l|r|r|r|r|r|r|r|r|r|}
\hline Regions & 1 & 2 & 3 & 4 & 5 & 6 & 7 & 8 & 9 \\
\hline & 0 & 30 & 26 & 27 & 27 & 21 & 36 & 131 & 98 \\
\hline
\end{tabular}


TWO-FOOT STANCE PERCENT BODY WEIGHT

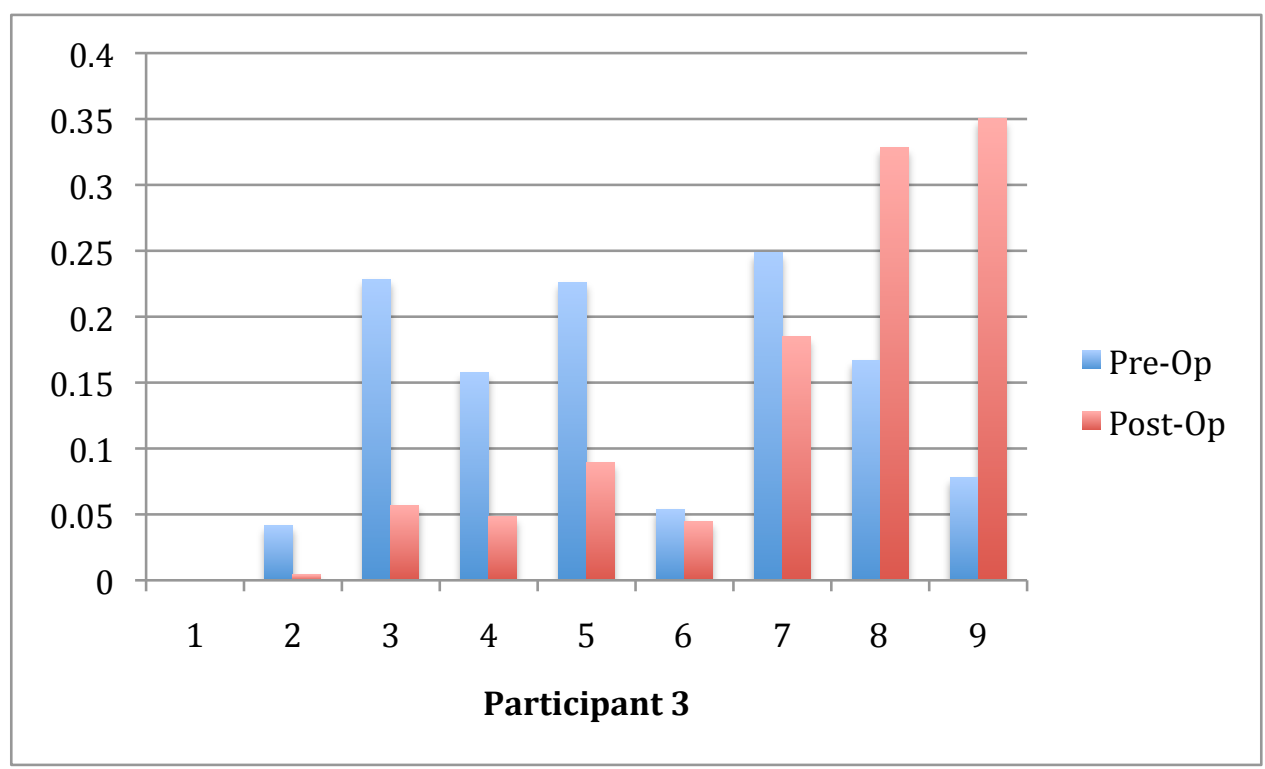

Figure A.13: Percent body weight of two-foot stance for the body weight distribution for afflicted foot

Pre-Op Percent Body Weight:

\begin{tabular}{|l|r|r|r|r|r|r|r|r|r|}
\hline Regions & 1 & 2 & 3 & 4 & 5 & 6 & 7 & 8 & 9 \\
\hline & 0.00 & 0.04 & 0.23 & 0.16 & 0.23 & 0.05 & 0.25 & 0.17 & 0.08 \\
\hline
\end{tabular}

Post-Op Percent Body Weight:

\begin{tabular}{|l|r|r|r|r|r|r|r|r|r|}
\hline Regions & 1 & 2 & 3 & 4 & 5 & 6 & 7 & 8 & 9 \\
\hline & 0.00 & 0.00 & 0.06 & 0.05 & 0.09 & 0.04 & 0.18 & 0.33 & 0.35 \\
\hline
\end{tabular}

Post-operatively there was a large increase in hindfoot loading in both peak pressure and percent body weight. With percent body weight, region 6 decreased postoperatively, but a very small amount. This participant does not follow the drastic changes usually seen in regions 3,56 , and 7 with a lateral shift toward 5 and 7 . 


\section{Arch Index:}

Arch index was calculated using the frame where the area of contact was at a peak during stance and walking trials after frame calibration. Calculations were made by using the formula from Murley et al by taking $B /(A+B+C)$ with the exclusion of the toes.

\section{TWO FOOT/ONE FOOT STANCE ARCH INDEX}

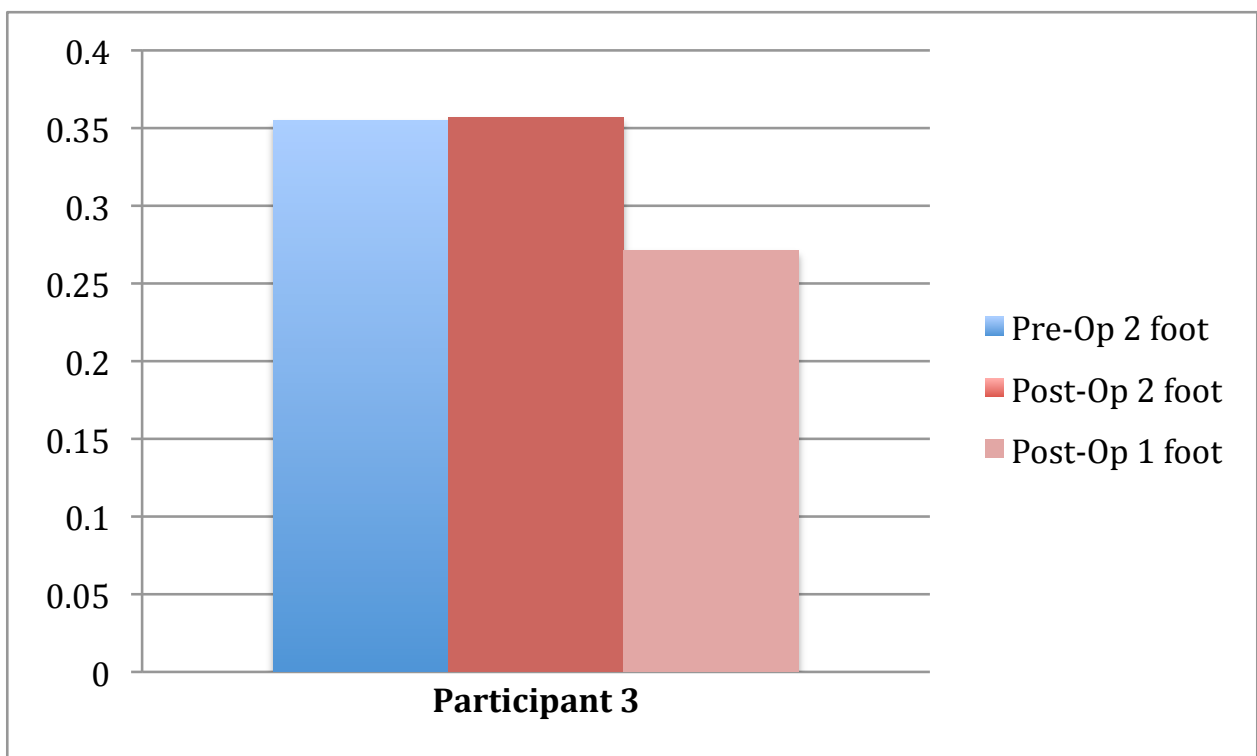

Figure A.14: Arch index comparison for stance of total peak area over time for twofoot stance and one foot stance

Pre-Op Two-Foot Arch Index: 0.36

Post-Op Two-Foot Arch Index: 0.36

Post-Op One-Foot Arch Index: 0.27

There was a dramatic decrease in arch index from one foot stance to two-foot stance post-operatively which may be due to the participant's distribution of her weight over both feet where compensation can occur. The one-foot arch index decreasing would suggest that the surgical correction was beneficial for correcting the arch in this participant. 


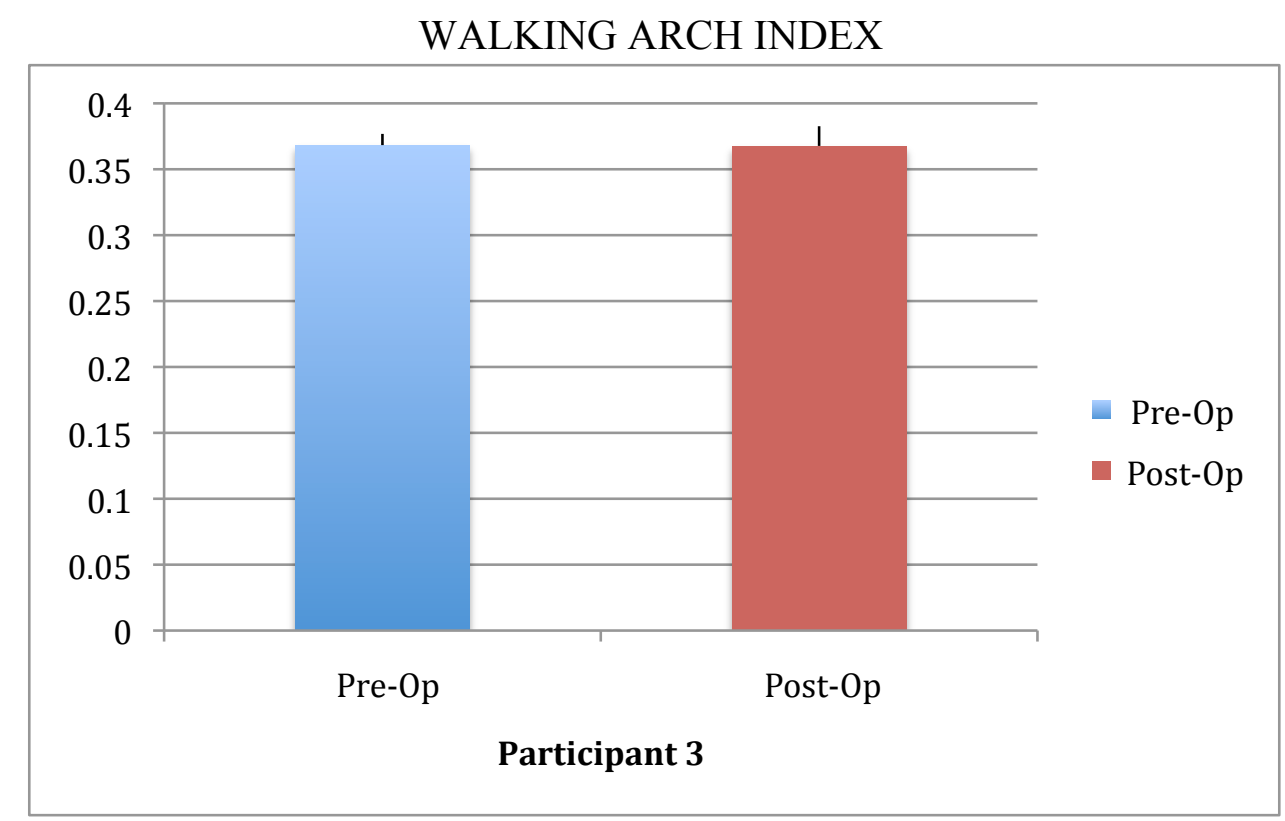

Figure A.15: Arch index measurements of the peak areas over time of three walking trials.

Pre-Op Arch index:

\begin{tabular}{|r|r|r|r|r|r|r|}
\hline Forefoot & Midfoot & Hindfoot & \multicolumn{1}{l|}{ Total } & $\begin{array}{l}\text { Arch } \\
\text { Index }\end{array}$ & $\begin{array}{l}\text { Average } \\
\text { AI }\end{array}$ & STD Dev \\
\hline 54 & 44 & 24 & 122 & 0.36 & & \\
\hline 50 & 46 & 26 & 122 & 0.38 & & \\
\hline 50 & 45 & 28 & 122 & 0.37 & 0.37 & 0.01 \\
\hline
\end{tabular}

Post-Op Arch index:

\begin{tabular}{|r|r|r|r|r|r|r|}
\hline Forefoot & Midfoot & Hindfoot & Total & $\begin{array}{r}\text { Arch } \\
\text { Index }\end{array}$ & $\begin{array}{l}\text { Average } \\
\text { AI }\end{array}$ & STD Dev \\
\hline 48 & 46 & 26 & 120 & 0.38 & & \\
\hline 42 & 41 & 31 & 114 & 0.36 & & \\
\hline 51 & 43 & 26 & 119 & 0.36 & 0.37 & 0.01 \\
\hline
\end{tabular}

With such a small decrease in arch index, from pre-op to post-op, this measurement is not a good indicator for the success of flatfoot for participant 3. According to normal levels, she would still be extremely flatfooted even after surgery when compared with a normal population. 
PARTICIPANT 4 (Left foot afflicted)

Participant 4 was first brought in on 12/21/2010 for plantar pressure analysis, radiographs, and motion analysis. Radiographs were taken first at third floor Gateway of MCV then participant traveled over to Broad St. to do motion analysis then plantar pressure protocol.

Participant 4 was brought back on 1/12/2012 for plantar pressure analysis and radiographs. No motion analysis was done. Plantar pressures were done in the Ambulatory Care Center in a spare protocol room. Radiographs were taken at third floor Gateway of MCV.

\section{Pre-Op Comments:}

The participant arrived and filled out the surveys while waiting for radiographs. She asked some questions about the survey where she was directed to answer however she interpreted the question. The surveys were finished at Broad St. where motion analysis was then commenced after a brief introduction about the protocol of motion testing. The participant walked approximately 20 times across the force plate one way which may have increased her pain level. It is to be noted that this particular participant also had a pain pump so the level of pain felt may have been increased or decreased comparatively to a normal participant.

Due to a computer glitch, the participant was asked to travel to VCU's Engineering East building where plantar pressure analysis was done in the Orthopedic Research Laboratory. Her weight was not measured at the lab because we did not have access to a scale, however she had weighed herself that morning therefore the calibration was based 
on her measurement. She took direction well and had good balance on the plantar pressure mat when asked to perform the one-foot stance measurement.

Three trials of each walking analysis of each foot were performed, and when compared to normal trials, the participant was visibly flatfooted. The stance trials also showed visible midfoot collapse.

\section{Post-Op Comments:}

After surgical correction via a medial calcaneal osteotomy (MCO) and tendon transfer, the participant returned for testing. The participant was pleased with her success of surgical correction where she said she could now easily move about. She took the surveys first and then was weighed for calibration. She then completed two-foot and onefoot stance trials without using the chair for extra balance. With the walking trials, she did about 15 passes as her whole foot image was not captured on each trial but did not need to rest between trials. No motion analysis was done.

\section{Surveys:}

The SF-36 is a determinate of overall health and quality of life on a percent based scale where if the participant is closer to $100 \%$ then the general health is good.

\section{$\underline{\text { SF-36 }}$}

Pre-Op: 34

Post-Op: 55

The FAOS (foot and ankle outcome score) is a determinate of the health of the foot and ankle as well as scaling the level of activities of daily living and overall health 
due to foot and ankle problems. This scale is out of 500, where the higher the total score, the less affected the foot and ankle issue is to the participant.

\section{FAOS}

Pre-Op: 74

Post-Op: 163

Participant 4 still shows significantly lower scores on both the SF-36 and the FAOS survey even with surgical correction. There is improvement by 21 percent for overall health and more than doubling of the foot and ankle outcome score, this participant is still not functioning at a higher level according to the surveys.

\section{Plantar Pressure images:}

Participant 4's individual plantar pressure pictures are shown at peak pressure over time (Figures A.16 and A.17). These images show the maximum amount of firing on each sensel over total contact time on the plantar pressure mat. For the stance measurement, the largest group of frame captures was used where the participant moved very little. The middle frame of the group was chosen as the most steady and used for calibration. Walking images taken are over the total time it took for the participant to go from heel strike to toe off. 
PRE-OP PLANTAR PRESSURE IMAGES

\section{$\underline{\text { PARTICIPANT } 4}$}
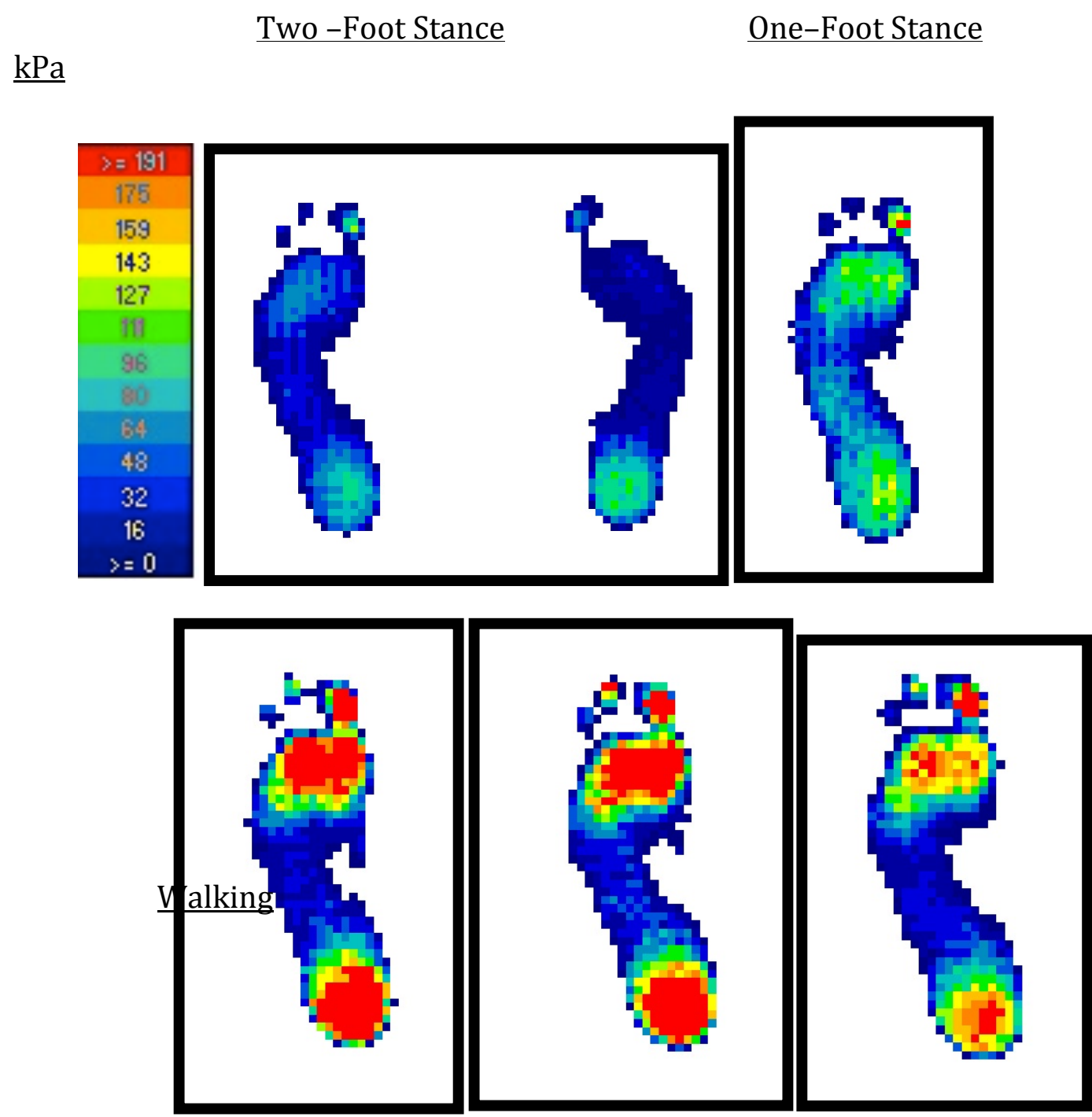

\section{$\underline{\text { Trial } 1}$}

Trial 2

$\underline{\text { Trial } 3}$

Figure A.16 : Pre-Op plantar pressure images for Participant 4 
POST-OP PLANTAR PRESSURE IMAGES

\section{PARTICIPANT 4}

$\underline{\mathrm{kPa}}$

Two -Foot Stance

$\underline{\text { One-Foot Stance }}$
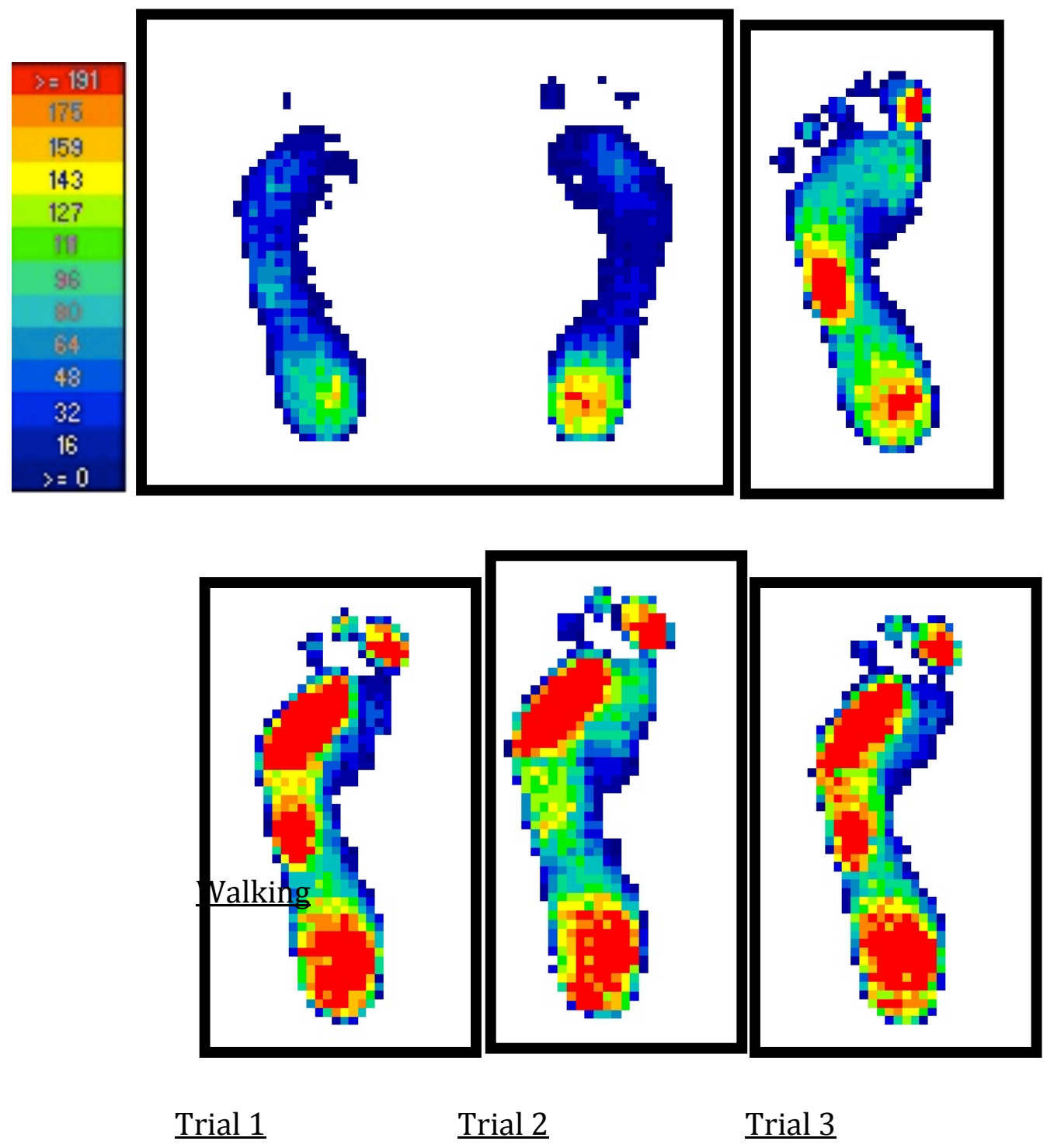

Figure A.17: Post-Op plantar pressure images for Participant 4

Walking: 
Three trials of each foot for walking analysis was performed and compared to preop analysis. Calibration of the plantar pressure mat was done by frame calibration where for each walking trial the middle frame of midstance was used.

\section{$\underline{\text { Peak Pressure: }}$}

Peak pressure is measured by taking the peak pressure in each region over the total time of the walking cycle. Each peak represents a distinct instant of time in the gait cycle for each region. This does not represent only one event in time, but nine separate events.

\section{WALKING PEAK PRESSURE}



Figure A.18: Peak pressure in each of the nine regions defined over the entire walking cycle averaged over three trials on the afflicted foot for Participant 4

Pre-Op Peak Pressures (kPa):

\begin{tabular}{|l|r|r|r|r|r|r|r|r|r|}
\hline Regions & 1 & 2 & 3 & 4 & 5 & 6 & 7 & 8 & 9 \\
\hline Average & 138 & 40 & 110 & 114 & 64 & 24 & 24 & 109 & 99 \\
\hline $\begin{array}{l}\text { Standard } \\
\text { Deviation }\end{array}$ & 21 & 6 & 5 & 10 & 13 & 3 & 5 & 3 & 4 \\
\hline
\end{tabular}


Post-Op Peak Pressures (kPa):

\begin{tabular}{|l|r|r|r|r|r|r|r|r|r|}
\hline Regions & 1 & 2 & 3 & 4 & 5 & 6 & 7 & 8 & 9 \\
\hline Average & 178 & 57 & 49 & 150 & 208 & 67 & 116 & 183 & 169 \\
\hline $\begin{array}{l}\text { Standard } \\
\text { Deviation }\end{array}$ & 59 & 7 & 23 & 7 & 17 & 10 & 19 & 18 & 13 \\
\hline
\end{tabular}

Comparing the pre-operative state to the post-operative state there is an increase in all pressure regions except regions 2 and 3. Region 3 decreasing post-operatively would suggest a lateral shift in pressure to regions 4 and 5 in the forefoot as shown in the graph. Medial pressure in region 6 did not decrease post-operatively, but region 7 has more of the medial distribution of pressure, as the difference between the regions is larger than pre-operatively.

\section{Percent Body Weight:}

The measurement of percent body weight was taken in each region by the maximum force in pounds in that region on the afflicted foot and dividing it by the total body weight of the participant. This was done so that the measurements can be compared across time if the bodyweight of the participant changed. The participant was weighed both pre-operatively (160 lbs) and post-operatively (164.2 lbs). 




Figure A.19: Maximum percent body weight over time of the walking trial for the average of three trials for the afflicted foot

Pre-Op Percent Body Weight:

\begin{tabular}{|l|r|r|r|r|r|r|r|r|r|}
\hline Regions & 1 & 2 & 3 & 4 & 5 & 6 & 7 & 8 & 9 \\
\hline Average & 0.17 & 0.03 & 0.28 & 0.17 & 0.24 & 0.02 & 0.11 & 0.28 & 0.33 \\
\hline $\begin{array}{l}\text { Standard } \\
\text { Deviation }\end{array}$ & 0.04 & 0.01 & 0.04 & 0.04 & 0.04 & 0.01 & 0.02 & 0.05 & 0.09 \\
\hline
\end{tabular}

Post-Op Percent Body Weight:

\begin{tabular}{|l|r|r|r|r|r|r|r|r|r|}
\hline Regions & 1 & 2 & 3 & 4 & 5 & 6 & 7 & 8 & 9 \\
\hline Average & 0.14 & 0.03 & 0.06 & 0.19 & 0.56 & 0.04 & 0.39 & 0.30 & 0.32 \\
\hline $\begin{array}{l}\text { Standard } \\
\text { Deviation }\end{array}$ & 0.04 & 0.01 & 0.04 & 0.01 & 0.06 & 0.01 & 0.08 & 0.02 & 0.01 \\
\hline
\end{tabular}

A large lateral shift is seen from region 3 to region 5 post-operatively as to be expected with flatfoot correction. Although there is not a dramatic decrease in region 6, region 7 shows that there is also a lateral shift in the midfoot with a large increase in percent body weight. 


\section{Stance:}

Trials were taken with the aid of a chair for balance (if needed) for both twofoot stance as well as one-foot stance. For one-foot stance, both afflicted foot and unafflicted foot were collected, however only the afflicted foot results are presented. Frame calibration was used to calibrate the trials by taking the middle frame of the collected response (10 seconds) for both two-foot and one-foot stance. .

\section{Two-Foot Stance:}

TWO-FOOT STANCE PEAK PRESSURE

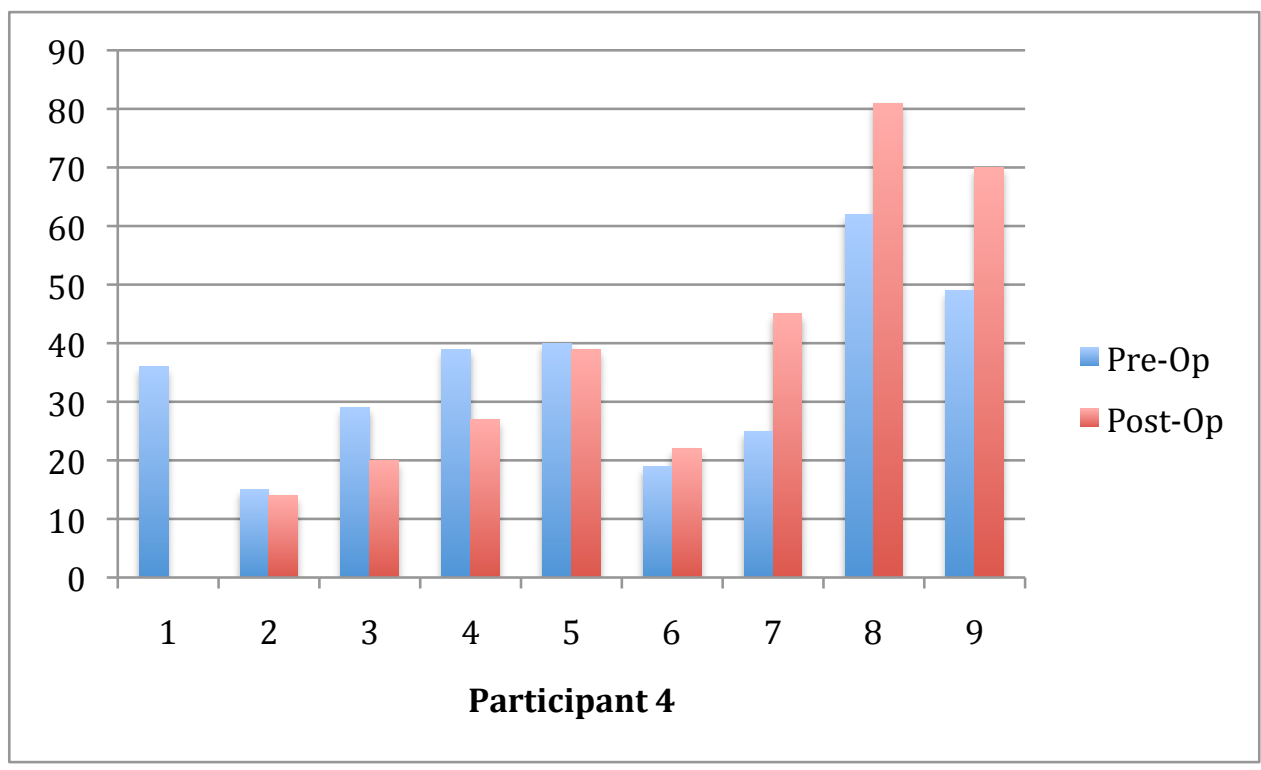

Figure A.20: Peak pressure measurements in regions over total stance time with two-foot stance for the afflicted foot

Pre-Op Peak Pressure $(\mathrm{kPa})$ :

\begin{tabular}{|l|r|r|r|r|r|r|r|r|r|}
\hline Regions & 1 & 2 & 3 & 4 & 5 & 6 & 7 & 8 & 9 \\
\hline & 36 & 15 & 29 & 39 & 40 & 19 & 25 & 62 & 49 \\
\hline
\end{tabular}

Post-Op Peak Pressure (kPa):

\begin{tabular}{|l|r|r|r|r|r|r|r|r|r|}
\hline Regions & 1 & 2 & 3 & 4 & 5 & 6 & 7 & 8 & 9 \\
\hline & 0 & 14 & 20 & 27 & 39 & 22 & 45 & 81 & 70 \\
\hline
\end{tabular}

There is an increase in heel loading post-operatively with a pressure profile.

This may be due to the fact that she did not use the chair for balance like she did 
pre-operatively. There is a lateral shift in the midfoot where region 7 bears more pressure than region 6 even though there is a slight increase in pressure post-op.

TWO-FOOT STANCE PERCENT BODY WEIGHT

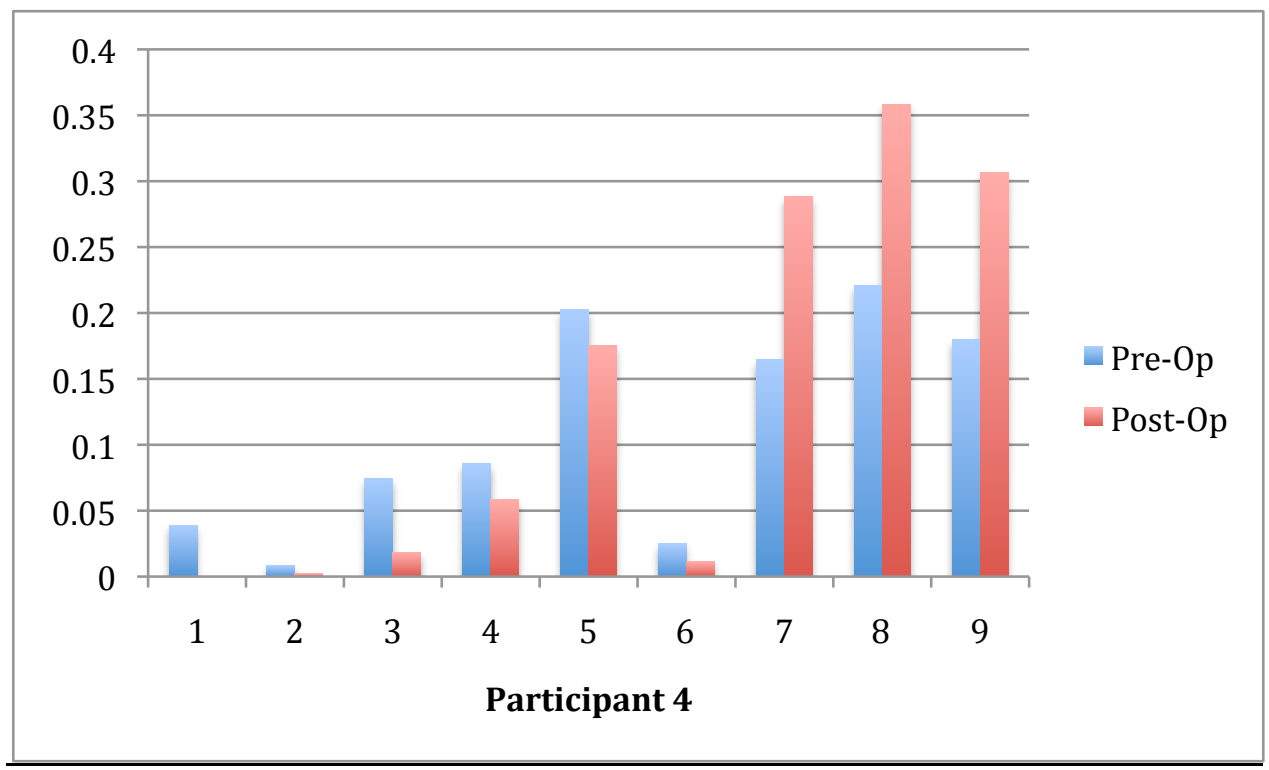

Figure A.21: Percent body weight of two-foot stance in each region for the body weight distribution of afflicted foot

Pre-Op Percent Body Weight:

\begin{tabular}{|l|r|r|r|r|r|r|r|r|r|}
\hline Regions & 1 & 2 & 3 & 4 & 5 & 6 & 7 & 8 & 9 \\
\hline & 0.04 & 0.01 & 0.07 & 0.09 & 0.20 & 0.02 & 0.16 & 0.22 & 0.18 \\
\hline
\end{tabular}

Post-Op Percent Body Weight:

\begin{tabular}{|l|r|r|r|r|r|r|r|r|r|}
\hline Regions & 1 & 2 & 3 & 4 & 5 & 6 & 7 & 8 & 9 \\
\hline & 0.00 & 0.00 & 0.02 & 0.06 & 0.18 & 0.01 & 0.29 & 0.36 & 0.31 \\
\hline
\end{tabular}

Percent body weight shows a dramatic decrease in both regions 3 and 6 , and an increase in regions 5 and 7 where both the forefoot and midfoot illustrate a lateral shift with surgical correction. There is also a large shift of percent body weight on the hindfoot because the participant did not use the chair in front to steady herself. 
One-foot Stance:

ONE FOOT STANCE PEAK PRESSURE

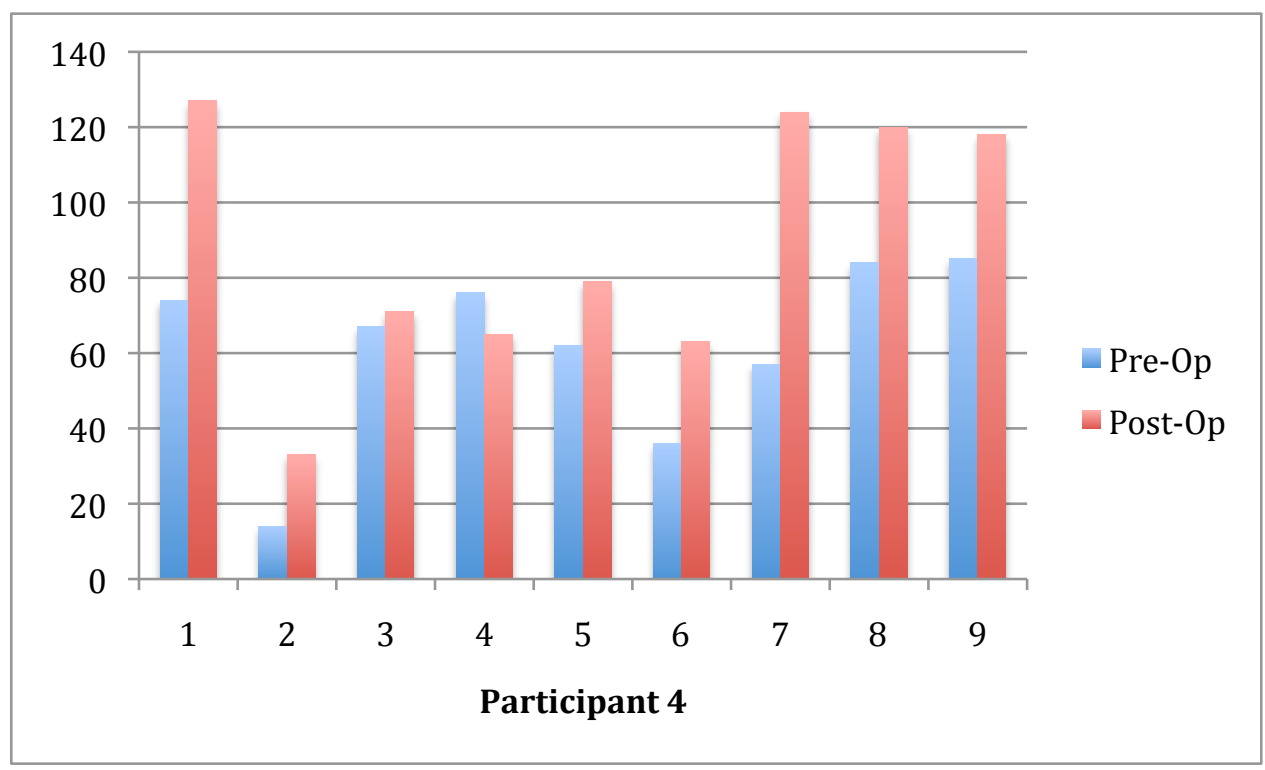

Figure A.22: Peak pressure measurements in the regions over total stance time with onefoot stance for the afflicted foot

Pre-Op Peak Pressure (kPa):

\begin{tabular}{|l|r|r|r|r|r|r|r|r|r|}
\hline Regions & 1 & 2 & 3 & 4 & 5 & 6 & 7 & 8 & 9 \\
\hline & 74 & 14 & 67 & 76 & 62 & 36 & 57 & 84 & 85 \\
\hline
\end{tabular}

Post-Op Peak Pressure (kPa):

\begin{tabular}{|l|r|r|r|r|r|r|r|r|r|}
\hline Regions & 1 & 2 & 3 & 4 & 5 & 6 & 7 & 8 & 9 \\
\hline & 127 & 33 & 71 & 65 & 79 & 63 & 124 & 120 & 118 \\
\hline
\end{tabular}

Pressure increased in all regions except for region 4 post-operatively. There is an opposite trend in the forefoot (regions 3-5) from pre-operatively to postoperatively with a heavier increase in region 5 , which would suggest a lateral shift in pressure. Region 7 has a dramatic increase more than doubling the pressure in the lateral midfoot. Although Region 6 also increases in the medial midfoot, there is a greater increase laterally. 


\section{ONE FOOT STANCE PERCENT BODY WEIGHT}

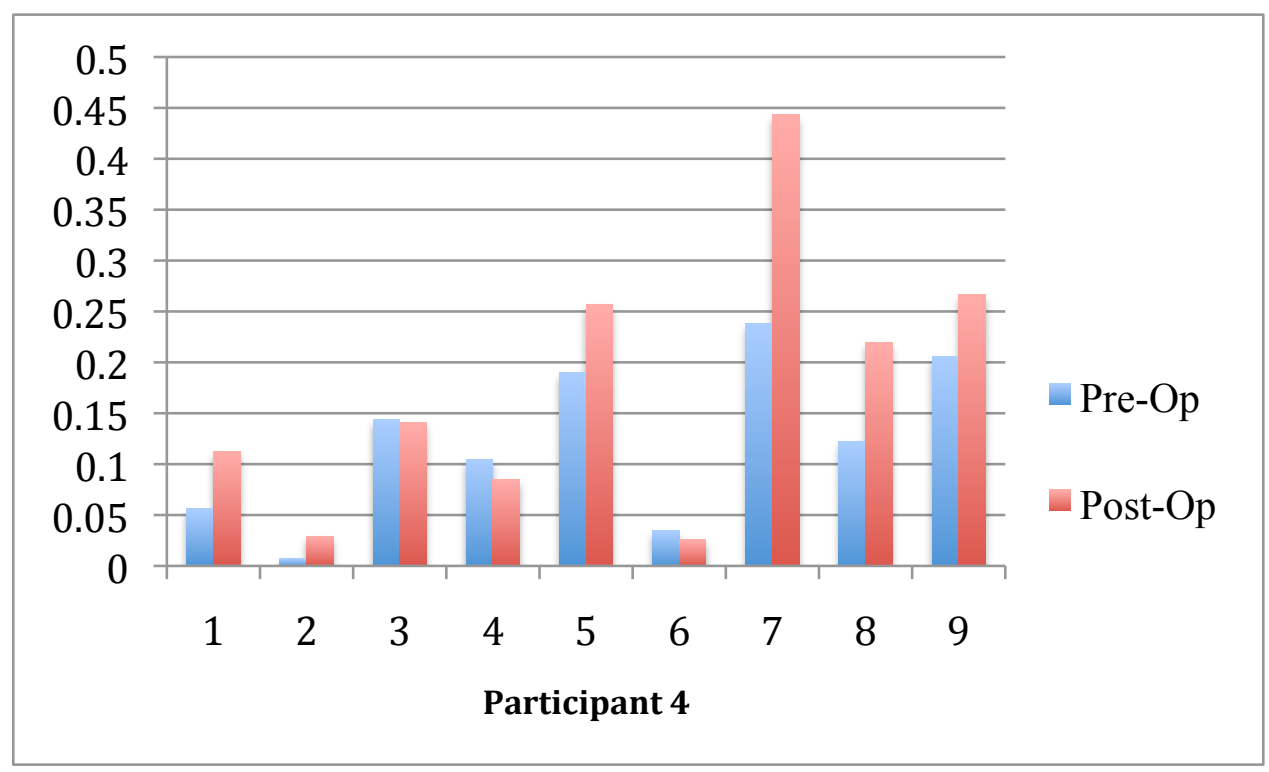

Figure A.23: Percent body weight measurements in regions over total stance time with one-foot stance where the afflicted foot was measured.

Pre- Op Percent Body Weight:

\begin{tabular}{|l|r|r|r|r|r|r|r|r|r|}
\hline Regions & 1 & 2 & 3 & 4 & 5 & 6 & 7 & 8 & 9 \\
\hline & 0.06 & 0.01 & 0.14 & 0.11 & 0.19 & 0.03 & 0.24 & 0.12 & 0.21 \\
\hline
\end{tabular}

Post-Op Percent Body Weight:

\begin{tabular}{|l|r|r|r|r|r|r|r|r|r|}
\hline Regions & 1 & 2 & 3 & 4 & 5 & 6 & 7 & 8 & 9 \\
\hline & 0.11 & 0.03 & 0.14 & 0.09 & 0.26 & 0.03 & 0.44 & 0.22 & 0.27 \\
\hline
\end{tabular}

Percent body weight measurement shows the decrease in regions 3,4 and 6 , with increases in regions 5 and 7, which is expected after surgical correction. This shift in percent body weight from medial to lateral from pre-op to post-op is what clinicians expect. Although there is a slight difference in medial and lateral hindfoot preoperatively, the difference shifts to a more even distribution of weight post-operatively. 


\section{Arch Index:}

Arch index was calculated using the frame where the area of contact was at a peak during stance and walking trials after frame calibration. Calculations were made by using the formula from Murley et al by taking $B /(A+B+C)$ with the exclusion of the toes.

\section{ARCH INDEX TWO-FOOT STANCE}

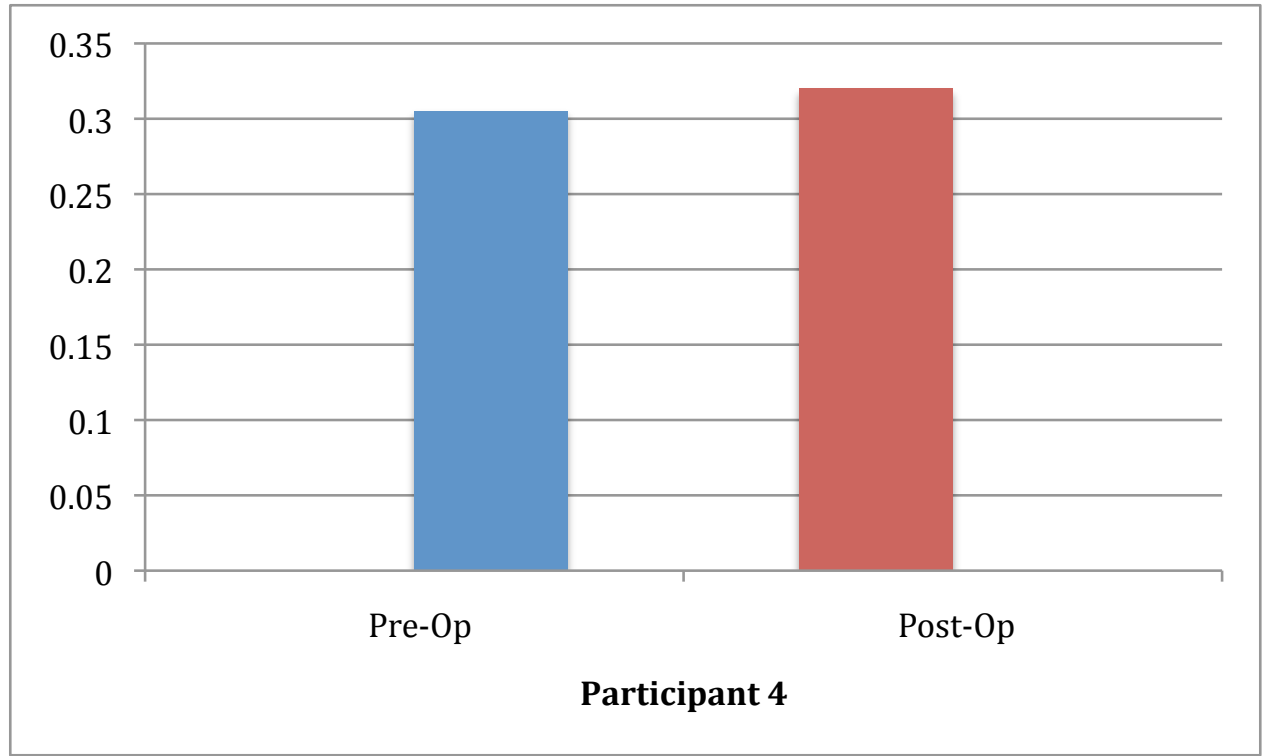

Figure A.24: Arch index comparison for stance of total peak area over time for two-foot stance

Pre-Op Arch Index: 0.30

Post-Op Arch Index: 0.31 


\section{ARCH INDEX ONE FOOT STANCE}

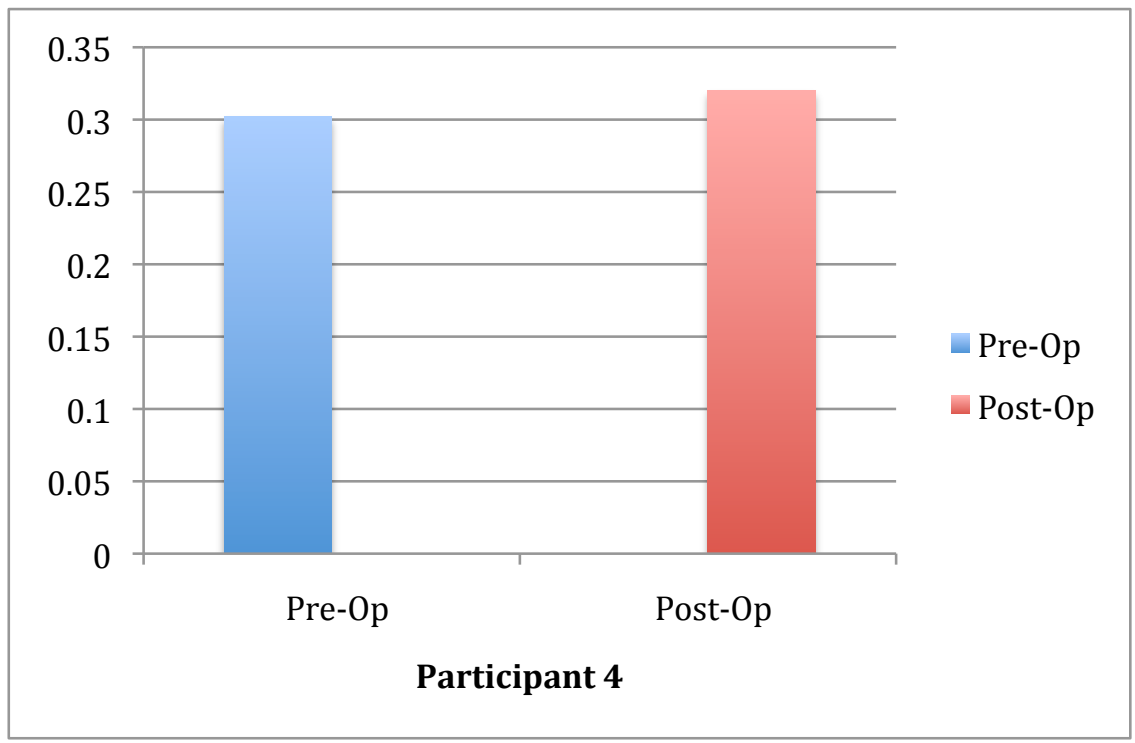

Figure A.25: Arch index comparison for stance of total peak area over time for one foot stance.

Pre-Op Arch Index: 0.30

Post-Op Arch Index: 0.29

ARCH INDEX WALKING

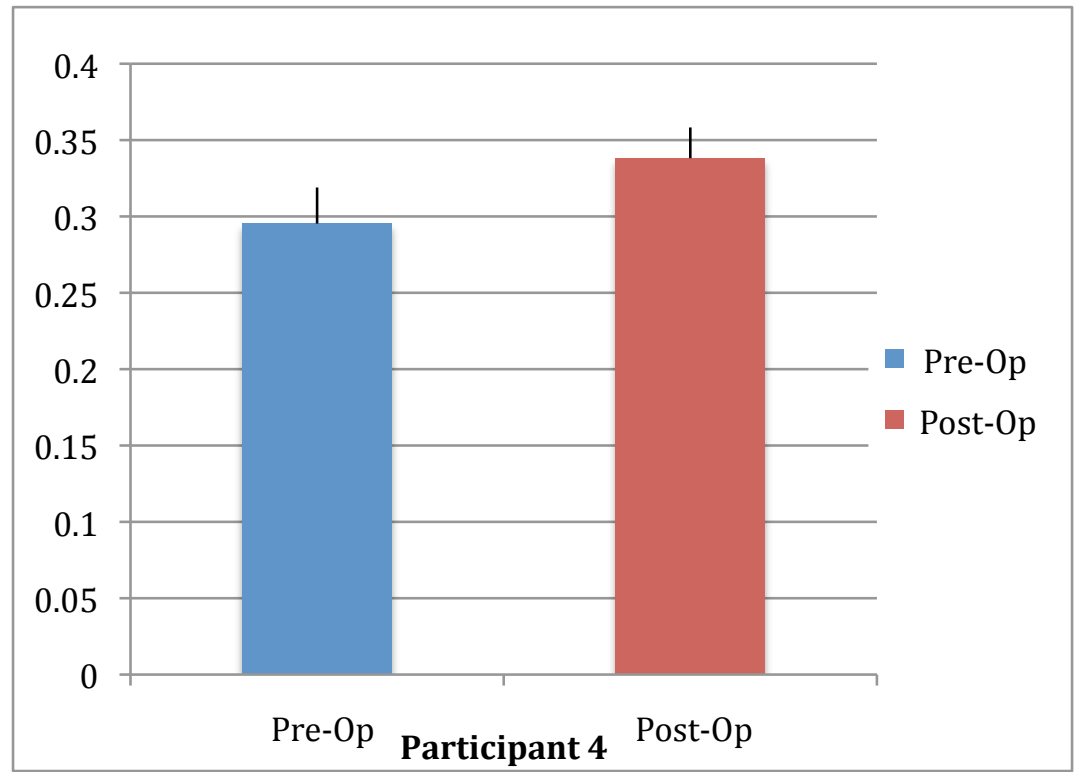

Figure A.26: Arch index measurements of the peak areas over time of three walking trials. 
Pre-Op Arch index:

\begin{tabular}{|r|r|r|r|r|r|r|}
\hline Forefoot & Midfoot & Hindfoot & Total & $\begin{array}{l}\text { Arch } \\
\text { Index }\end{array}$ & $\begin{array}{l}\text { Average } \\
\text { AI }\end{array}$ & STD Dev \\
\hline 44 & 36 & 31 & 111 & 0.32 & & \\
\hline 49 & 32 & 31 & 112 & 0.29 & & \\
\hline 46 & 30 & 32 & 108 & 0.28 & 0.30 & 0.02 \\
\hline
\end{tabular}

Post-Op Arch index:

\begin{tabular}{|r|r|r|r|r|l|r|}
\hline Forefoot & Midfoot & Hindfoot & Total & $\begin{array}{r}\text { Arch } \\
\text { Index }\end{array}$ & $\begin{array}{l}\text { Average } \\
\text { AI }\end{array}$ & STD Dev \\
\hline 38 & 35 & 28 & 100 & 0.35 & & \\
\hline 43 & 37 & 26 & 106 & 0.35 & & \\
\hline 40 & 32 & 29 & 101 & 0.32 & 0.34 & 0.02 \\
\hline
\end{tabular}

Arch index may not be a great measurement for Participant 4's success of surgery as the arch index increased in two-foot stance as well as walking. A slight decrease in arch index was observed during the one-foot stance, however this may be due to the fact that the participant was not leaning forward to balance with the chair post-operatively, which would cause a shift in force to display a more normal state of stance where the distribution would rely less on the forefoot and more on the hindfoot.

\section{PARTICIPANT 6 (Right foot afflicted)}

Participant 6 was first brought in on 5/5/2011 for plantar pressure analysis, radiographs, and motion analysis. Plantar pressure protocol was done first at the Ambulatory Care Center and then radiographs were taken first at third floor Gateway of MCV then the participant traveled over to Broad St. to do motion analysis.

Participant 6 was brought back on 6/5/2012 for plantar pressure analysis and radiographs. No motion analysis was done. Plantar pressures were done in the 
Ambulatory Care Center in a spare protocol room. Radiographs were taken at third floor Gateway of MCV.

\section{Pre-Op Comments:}

The participant arrived and it was extremely difficult for her to walk and stand on the plantar pressure mat. She heavily relied upon the use of the chair when standing and did not have a normal foot shape according to plantar pressure. She leaned more laterally on her foot so that it "did not hurt" her to do so. Her gait pattern was a bit wobbly as she relied upon the uninjured foot where one foot on the mat for a walking trial was recorded. Two-foot stance was done as well as one-foot stance at a higher frequency to record more frames of data per second.

Three trials of each walking analysis of each foot were performed, and when compared to normal trials, the participant looked extremely laterally skewed, with no sign of any toes. The stance trials also showed lateral movement to compensate for her pain.

\section{Post-Op Comments:}

After surgical correction via a medial calcaneal osteotomy (MCO) and tendon transfer, the participant returned for testing. The participant arrived on a rainy day and said that although she was stiff but she was feeling much better. She sat down to take the surveys and left some blank on the sports section because it did not apply to her. She also mentioned that she would be coming back next week as she talked about "feeling the screw in her heel" which may affect her gait. She also said that she was "afraid" to walk 
or turn her "bad foot" because she did not want to hurt it again. Standing, she clearly favored one foot over the other, however did not completely utilize the chair in front of her. She did have to do many more walking trials than pre-op because of missing the mat but she happily obliged with no rest between. No motion analysis was done. Both twofoot and one-foot stance was captured and analyzed.

\section{Surveys:}

The SF-36 is a determinate of overall health and quality of life on a percent based scale where if the participant is closer to $100 \%$ then the general health is good.

$\underline{\mathrm{SF}-36}$

Pre-Op: 49

Post-Op: 47

Participant 6 was the only participant to have a decreasing score on the general health survey. This may be due to the fact that she is still having foot complications as well as gaining 20 pounds after surgery.

The FAOS (foot and ankle outcome score) is a determinate of the health of the foot and ankle as well as scaling the level of activities of daily living and overall health due to foot and ankle problems. This scale is out of 500, where the higher the total score, the less affected the foot and ankle issue is to the participant.

$\underline{\text { FAOS }}$

Pre-Op: 213

Post-Op: 251

Although it was not a dramatic increase in the scores, this may be due to the fact that the participant is still having foot pain and complications. 


\section{Plantar pressure images:}

Participant 6's individual plantar pressure images are shown at peak pressure over time (Figures A.26 and A.27). These images show the maximum amount of firing on each sensel over total time on the plantar pressure mat. For the stance measurement the largest group of frame captures were used where the participant moved very little. The middle frame of the group was chosen as the most steady and used for calibration.

Walking images shown are the total time it took for the participant to go from heel strike to toe off. 


\begin{tabular}{|c|c|c|}
\hline & P PLANTAR PRESS & IMAGES \\
\hline PARIICIPANI 6 & Two -Foot Stance & One-Foot Stance \\
\hline
\end{tabular}

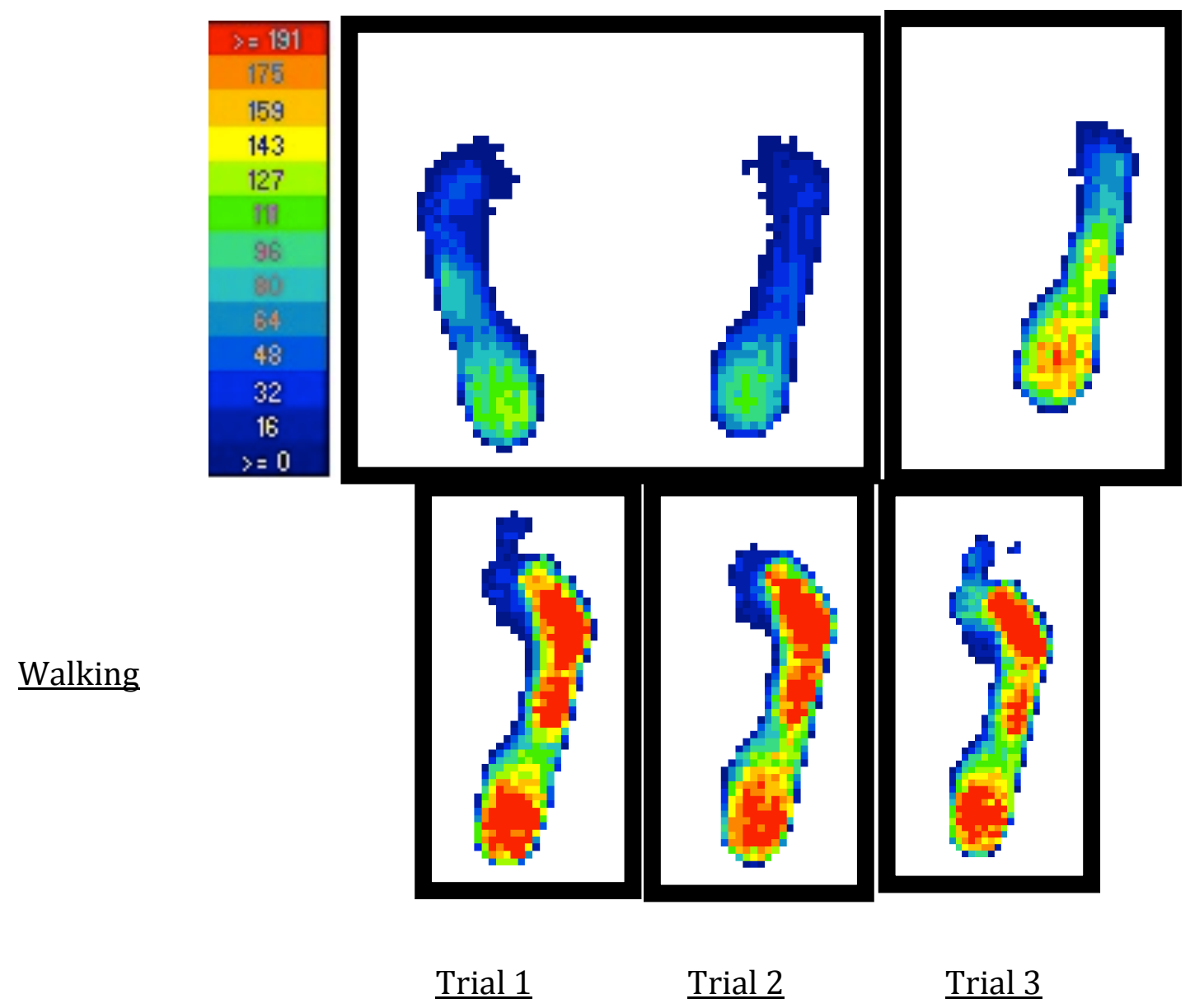

Figure A.26: Pre-Op plantar pressure images for Participant 6 


\section{PARTICIPANT 6}

\section{POST-OP PLANTAR PRESSURE IMAGES}

$\underline{\mathrm{kPa}}$

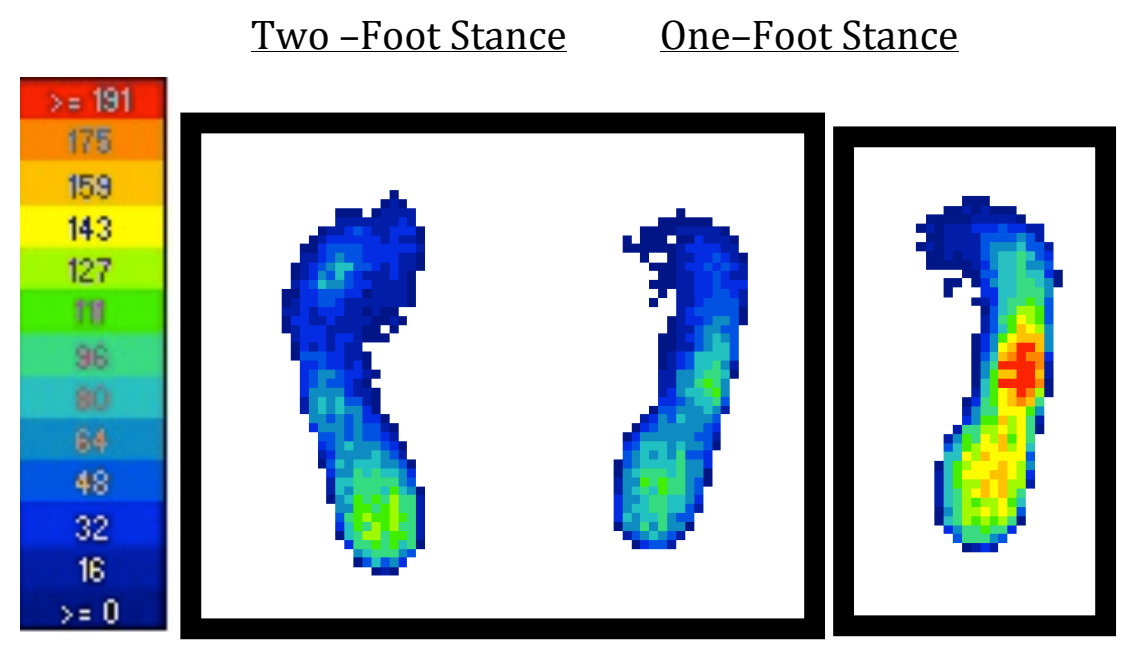

Walking

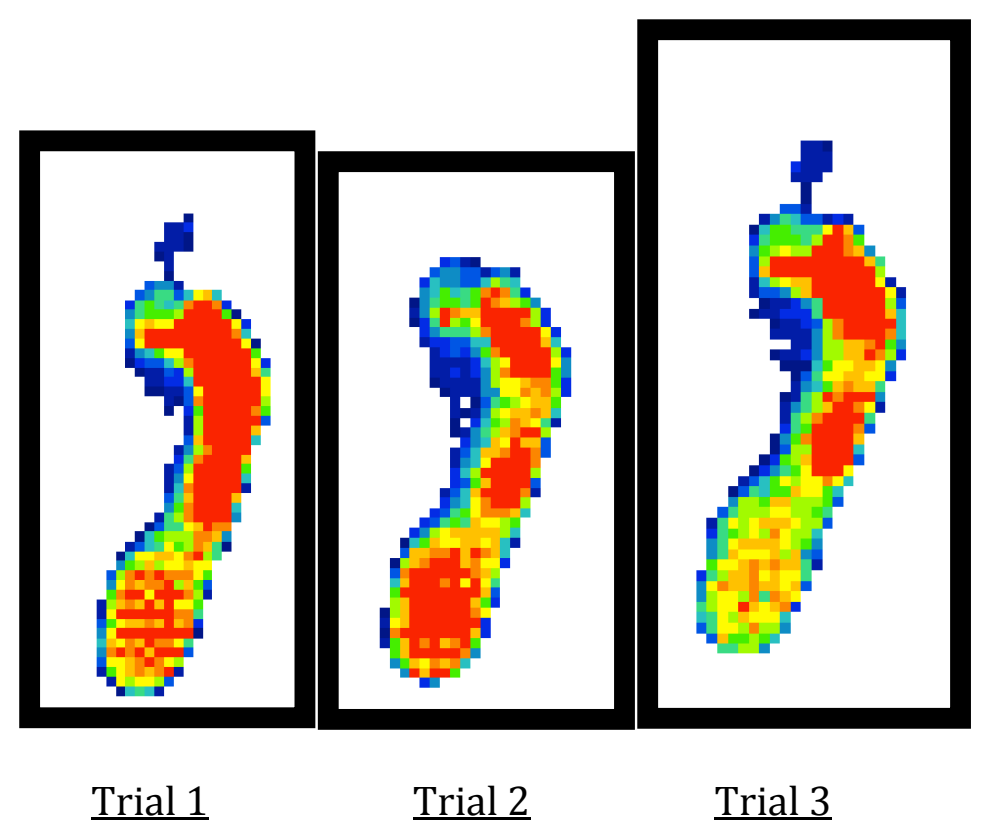

Figure A.27: Post-Op plantar pressure images for Participant 6

\section{Walking:}

Three trials of each foot for walking analysis was performed and compared to preop analysis. Calibration of the plantar pressure mat was done by frame calibration where for each walking trial the middle frame of midstance was used. 


\section{Peak Pressure:}

Peak pressure is measured by taking the peak pressure in each region over the total time of the walking cycle. Each peak represents a distinct instant of time in the gait cycle for each region. This does not represent only one event in time, but nine separate events.

\section{WALKING PEAK PRESSURE}

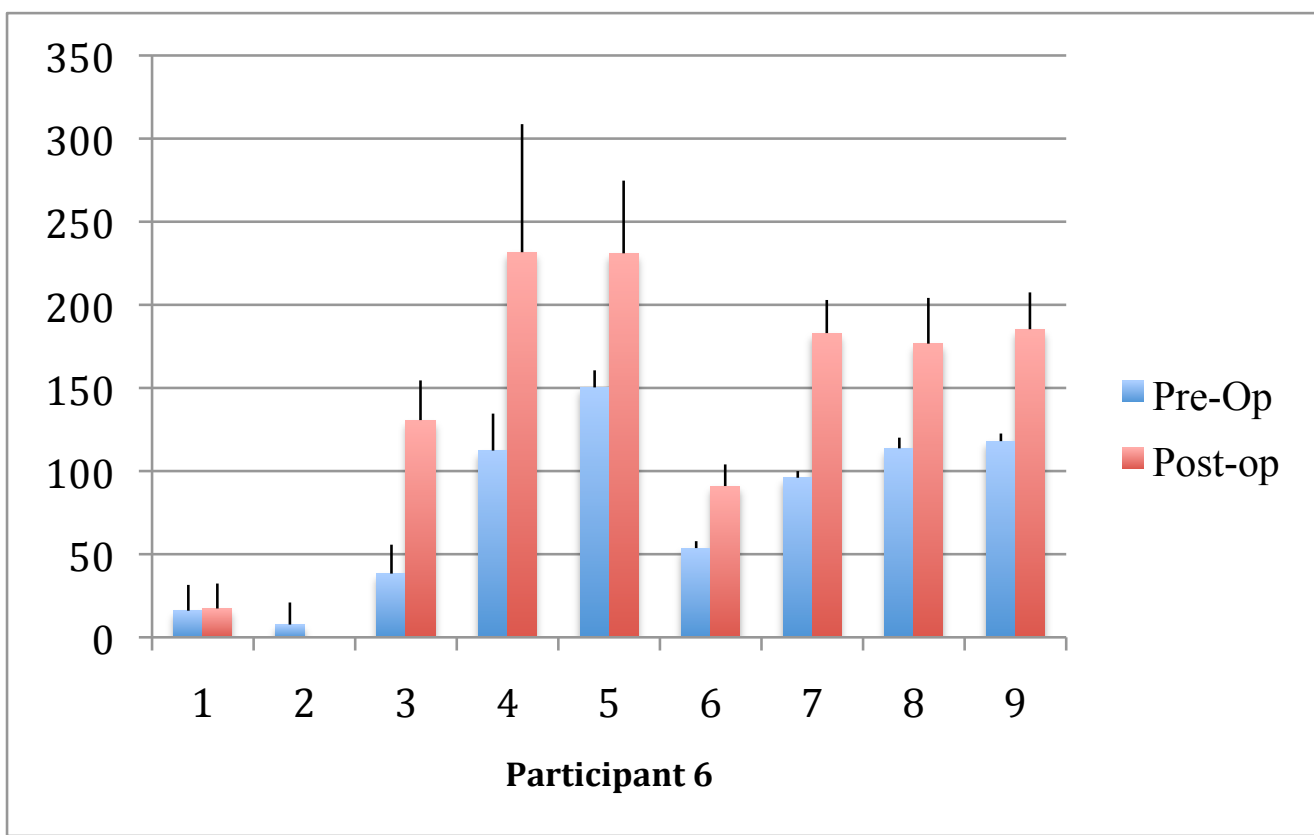

Figure A.28: Peak pressure in each of the nine regions defined over the entire walking cycle averaged over three trials of the afflicted foot for Participant 6 .

Pre-Op Peak Pressures (kPa):

\begin{tabular}{|l|r|r|r|r|r|r|r|r|r|}
\hline Regions & 1 & 2 & 3 & 4 & 5 & 6 & 7 & 8 & 9 \\
\hline Average & 16 & 8 & 38 & 112 & 150 & 54 & 96 & 114 & 118 \\
\hline $\begin{array}{l}\text { Standard } \\
\text { Deviation }\end{array}$ & 16 & 13 & 17 & 22 & 10 & 4 & 4 & 6 & 5 \\
\hline
\end{tabular}

Post-Op Peak Pressures (kPa):

\begin{tabular}{|l|r|r|r|r|r|r|r|r|r|}
\hline Regions & 1 & 2 & 3 & 4 & 5 & 6 & 7 & 8 & 9 \\
\hline Average & 17 & 0 & 131 & 232 & 231 & 91 & 183 & 177 & 185 \\
\hline $\begin{array}{l}\text { Standard } \\
\text { Deviation }\end{array}$ & 15 & 0 & 24 & 77 & 44 & 13 & 20 & 28 & 22 \\
\hline
\end{tabular}


Comparing the pre-op pressures to the post-op pressures there is a general shift in pressure toward the front of the foot after surgery. Also a lateral shift can be observed, especially in regions four and five. Although all the post-op pressures increased, there is a larger shift of pressure both forward and laterally.

\section{Percent Body Weight:}

The measurement of percent body weight was taken in each region by the maximum force in pounds in that region on the afflicted foot and dividing it by the total body weight of the participant. This was done so that the measurements can be compared across time if the bodyweight of the participant changed. The participant was weighed both pre-operatively (192.5 lbs) and post-operatively (211 lbs).

\section{WALKING PERCENT BODY WEIGHT}

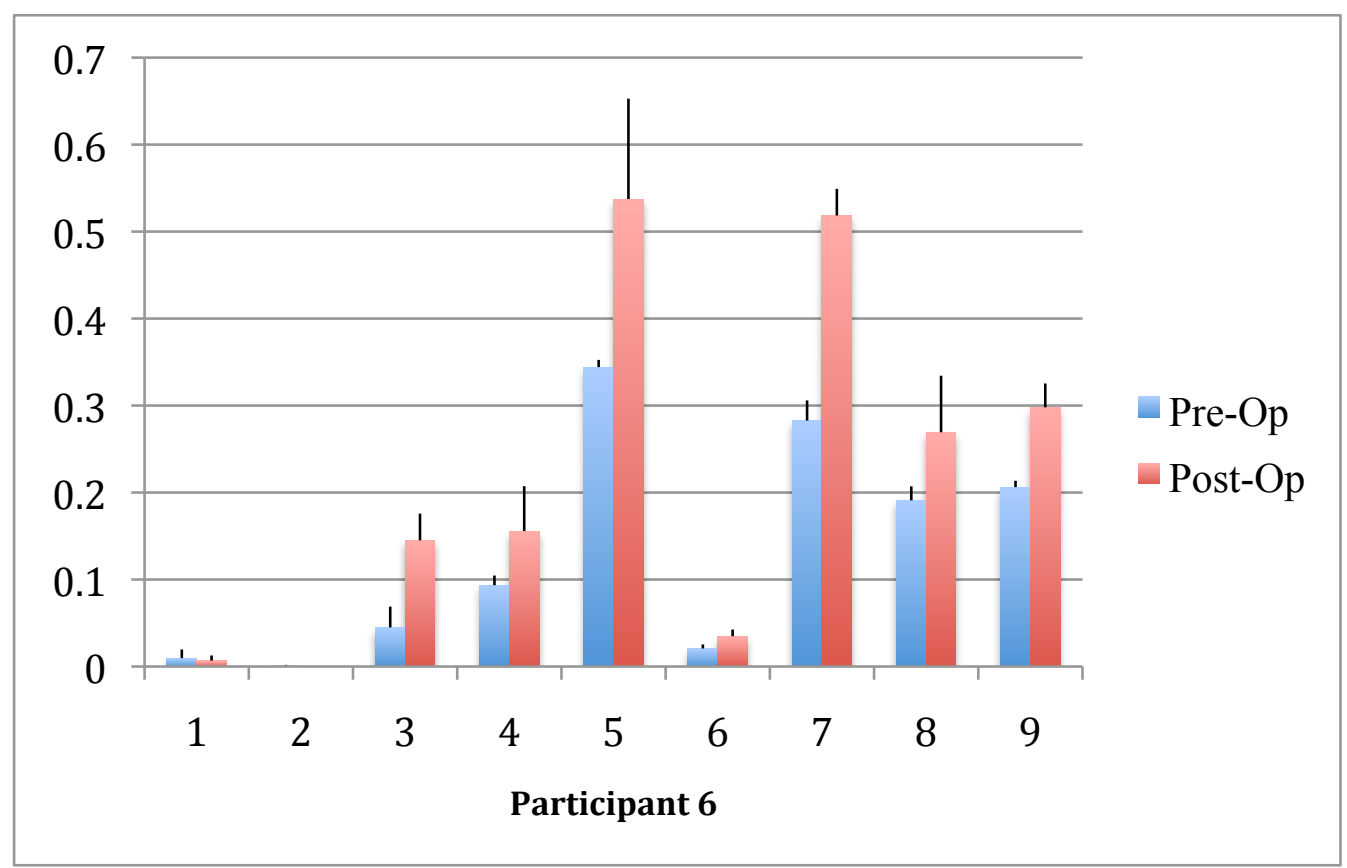

Figure A.29: Maximum percent body weight over time of the walking trial for the average of three trials of the afflicted foot for Participant 6 . 
Pre-Op Percent Body Weight:

\begin{tabular}{|l|r|r|r|r|r|r|r|r|r|}
\hline Regions & 1 & 2 & 3 & 4 & 5 & 6 & 7 & 8 & 9 \\
\hline Average & 0.01 & 0.00 & 0.05 & 0.09 & 0.34 & 0.02 & 0.28 & 0.19 & 0.21 \\
\hline $\begin{array}{l}\text { Standard } \\
\text { Deviation }\end{array}$ & 0.01 & 0.00 & 0.02 & 0.01 & 0.01 & 0.00 & 0.02 & 0.02 & 0.01 \\
\hline
\end{tabular}

Post-Op Percent Body Weight:

\begin{tabular}{|l|r|r|r|r|r|r|r|r|r|}
\hline Regions & 1 & 2 & 3 & 4 & 5 & 6 & 7 & 8 & 9 \\
\hline Average & 0.01 & 0.00 & 0.15 & 0.16 & 0.54 & 0.04 & 0.52 & 0.27 & 0.30 \\
\hline $\begin{array}{l}\text { Standard } \\
\text { Deviation }\end{array}$ & 0.01 & 0.00 & 0.03 & 0.05 & 0.12 & 0.01 & 0.03 & 0.06 & 0.03 \\
\hline
\end{tabular}

Percent body weight measurement showed a lateral shift in regions 5 and 7 where more of the weight is being distributed laterally in both the forefoot and midfoot.

Although Region 6 should decrease, this may be due to the participants slighted gait and favoring of the lateral side of her foot pre-operatively and thus with the correction she can now actually use her medial midfoot while walking.

The standard deviation measurement in both peak pressure and percent body weight is because there were three trials of walking on the afflicted foot that were averaged. There are standard deviation bars that are apparent in Figures A.28 and A.29.

\section{Stance:}

Trials were taken with the aid of a chair for balance (if needed) for both two-foot stance as well as one-foot stance. For one-foot stance, both afflicted foot and un-afflicted foot were collected, however only the afflicted foot results are presented. Frame calibration was used to calibrate the trials by taking the middle frame of the collected response (10 seconds). 
Two-Foot Stance:

TWO-FOOT STANCE PEAK PRESSURE

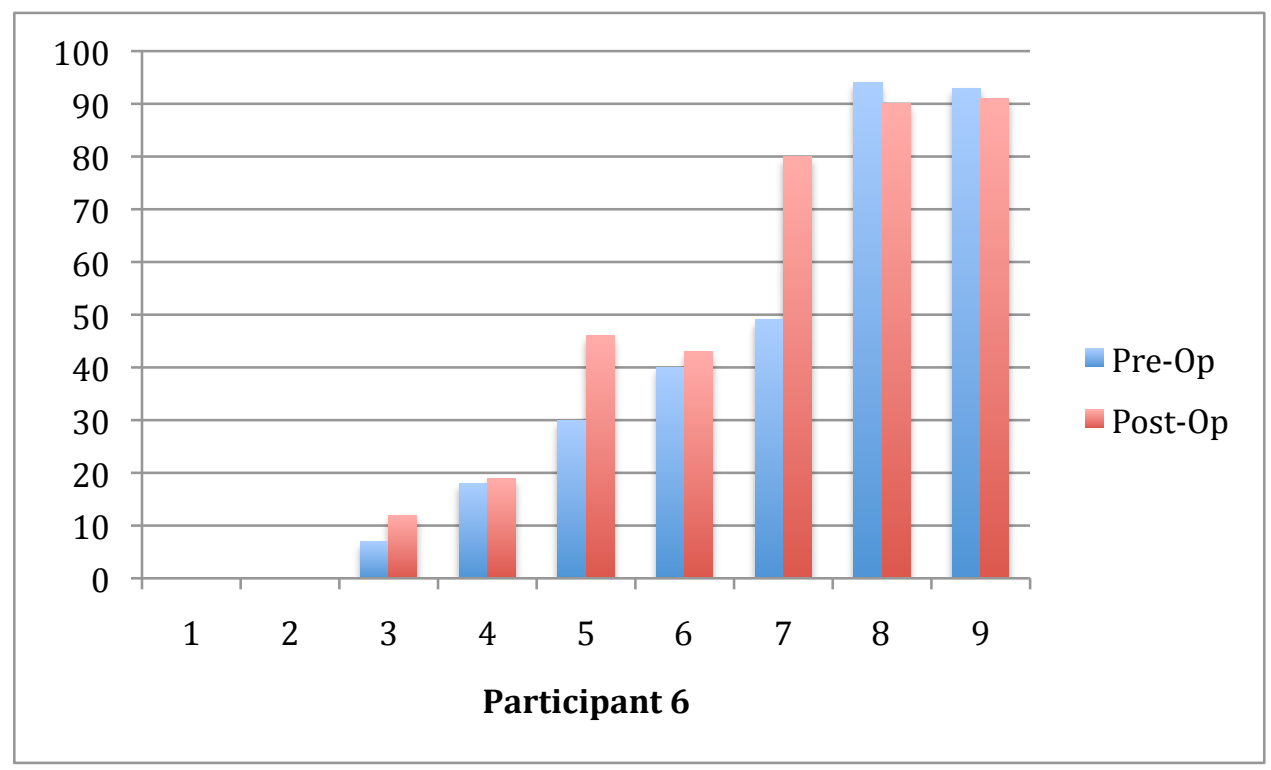

Figure A.30: Peak pressure measurements in regions over total stance time with two-foot stance for the afflicted foot

Pre-Op Pressure $(\mathrm{kPa})$ :

\begin{tabular}{|l|r|r|r|r|r|r|r|r|r|}
\hline Regions & 1 & 2 & 3 & 4 & 5 & 6 & 7 & 8 & 9 \\
\hline & 0 & 0 & 7 & 18 & 30 & 40 & 49 & 94 & 93 \\
\hline
\end{tabular}

Post-Op Pressure $(\mathrm{kPa})$ :

\begin{tabular}{|l|l|l|r|r|r|r|r|r|r|}
\hline Regions & 1 & 2 & 3 & 4 & 5 & 6 & 7 & 8 & 9 \\
\hline & 0 & 0 & 12 & 19 & 46 & 43 & 80 & 90 & 91 \\
\hline
\end{tabular}

In general, the pressure increased on 2-foot stance in the post-op trial. This may be due to the fact that the participant is more comfortable putting weight on her afflicted foot after corrective surgery. Regions 5 and 7 show a dramatic increase in lateral pressure. 
TWO-FOOT STANCE PERCENT BODY WEIGHT

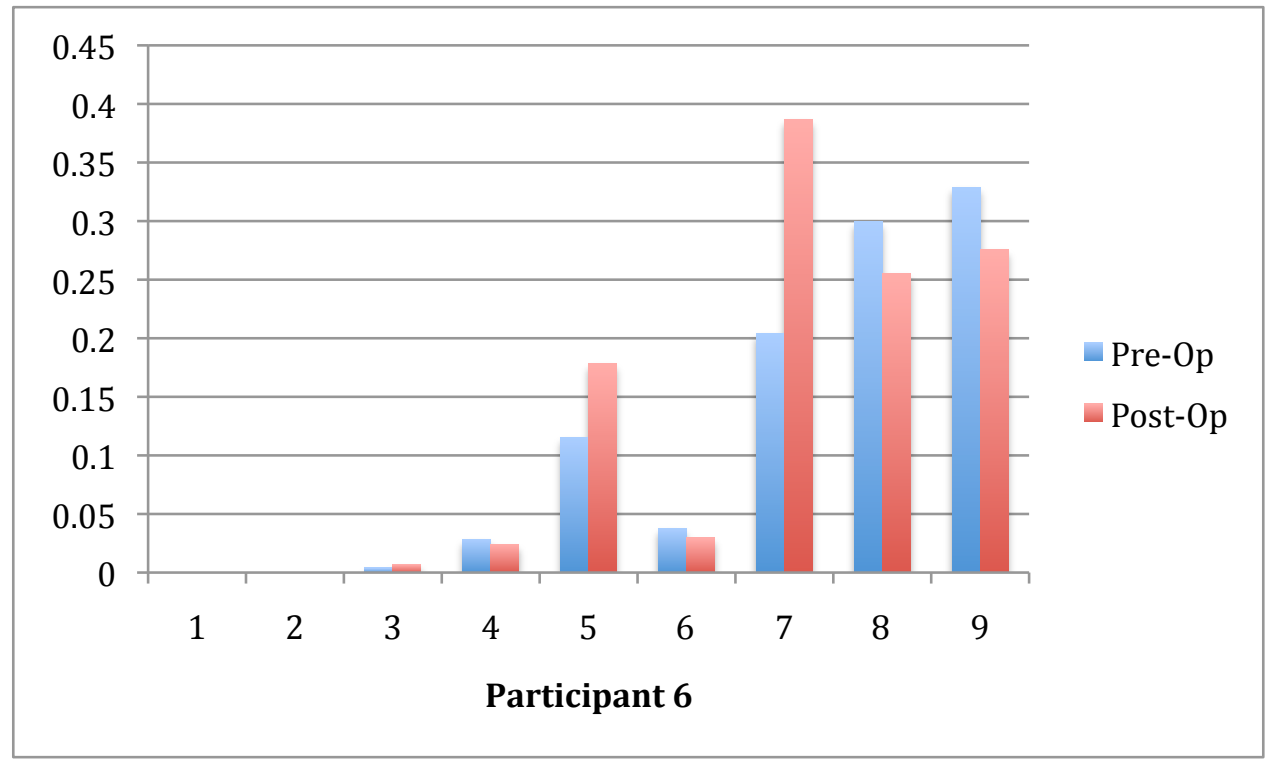

Figure A.31: Percent body weight of two-foot stance for the body weight distribution of afflicted foot

Pre-Op Percent Body Weight:

\begin{tabular}{|l|r|r|r|r|r|r|r|r|r|}
\hline Regions & 1 & 2 & 3 & 4 & 5 & 6 & 7 & 8 & 9 \\
\hline & 0.00 & 0.00 & 0.00 & 0.03 & 0.12 & 0.04 & 0.20 & 0.30 & 0.33 \\
\hline
\end{tabular}

Post-Op Percent Body Weight:

\begin{tabular}{|l|r|r|r|r|r|r|r|r|r|}
\hline Regions & 1 & 2 & 3 & 4 & 5 & 6 & 7 & 8 & 9 \\
\hline & 0.00 & 0.00 & 0.01 & 0.02 & 0.18 & 0.03 & 0.39 & 0.26 & 0.28 \\
\hline
\end{tabular}

Percent body weight shows a similar result to the pressure readings where the participant shows significant lateral shifting; especially in regions 5 and 7 (the lateral portion of the midfoot), which can be assumed, is from the corrective surgery.

Interestingly, region 6 does decrease with the percent body weight, which may be due to the fact that the $30 \mathrm{lb}$ increase in participant weight post-operatively may have affected the pressure reading. 
One-Foot Stance:

ONE FOOT STANCE PEAK PRESSURE



Figure A.32: Peak pressure measurements in regions over total stance time with one-foot stance for the afflicted foot.

Pre-Op Peak Pressure $(\mathrm{kPa})$ :

\begin{tabular}{|l|l|l|l|l|r|r|r|r|r|}
\hline Regions & 1 & 2 & 3 & 4 & 5 & 6 & 7 & 8 & 9 \\
\hline & 0 & 0 & 0 & 0 & 64 & 87 & 139 & 176 & 191 \\
\hline
\end{tabular}

Post-Op Peak Pressure (kPa):

\begin{tabular}{|l|l|l|r|r|r|r|r|r|r|}
\hline Regions & 1 & 2 & 3 & 4 & 5 & 6 & 7 & 8 & 9 \\
\hline & 0 & 0 & 18 & 48 & 96 & 60 & 171 & 118 & 153 \\
\hline
\end{tabular}

Just as in 2-foot stance, there was a lateral and forward shift of pressures where the hindfoot had less pressure in post-op results. This also may be due to the fact that when standing on one foot the participant was more likely to hold onto the chair for balance help. This was the case for P6 where that would create a forward shift in pressure. The participant clearly favors the lateral side in one-foot stance. 


\section{ONE FOOT STANCE PERCENT BODY WEIGHT}



Figure A.33: Percent body weight measurements in regions over total stance time with one-foot stance for the afflicted foot.

Pre-Op Percent Body Weight:

\begin{tabular}{|l|r|r|r|r|r|r|r|r|r|}
\hline Regions & 1 & 2 & 3 & 4 & 5 & 6 & 7 & 8 & 9 \\
\hline & 0.00 & 0.00 & 0.00 & 0.00 & 0.18 & 0.04 & 0.36 & 0.30 & 0.34 \\
\hline
\end{tabular}

Post-Op Percent Body Weight:

\begin{tabular}{|l|r|r|r|r|r|r|r|r|r|}
\hline Regions & 1 & 2 & 3 & 4 & 5 & 6 & 7 & 8 & 9 \\
\hline & 0.00 & 0.00 & 0.02 & 0.04 & 0.17 & 0.04 & 0.43 & 0.16 & 0.28 \\
\hline
\end{tabular}

Similar trends to one-foot pressure were seen in one-foot percent body weight. A lateral forward shift off the heels was also seen here where the largest change is in the region 7 where the lateral shift occurs. Also the participant finally feels comfortable enough after surgery to stand all the way on her foot instead of skewed to the lateral side as indicated by readings in regions 3 and 4 post-operatively.

\section{Arch Index:}

Arch index was calculated using the frame where the area of contact was at a peak during stance and walking trials after frame calibration. Calculations were made by using the formula from Murley et al by taking $B /(A+B+C)$ with the exclusion of the toes. 
TWO FOOT STANCE ARCH INDEX

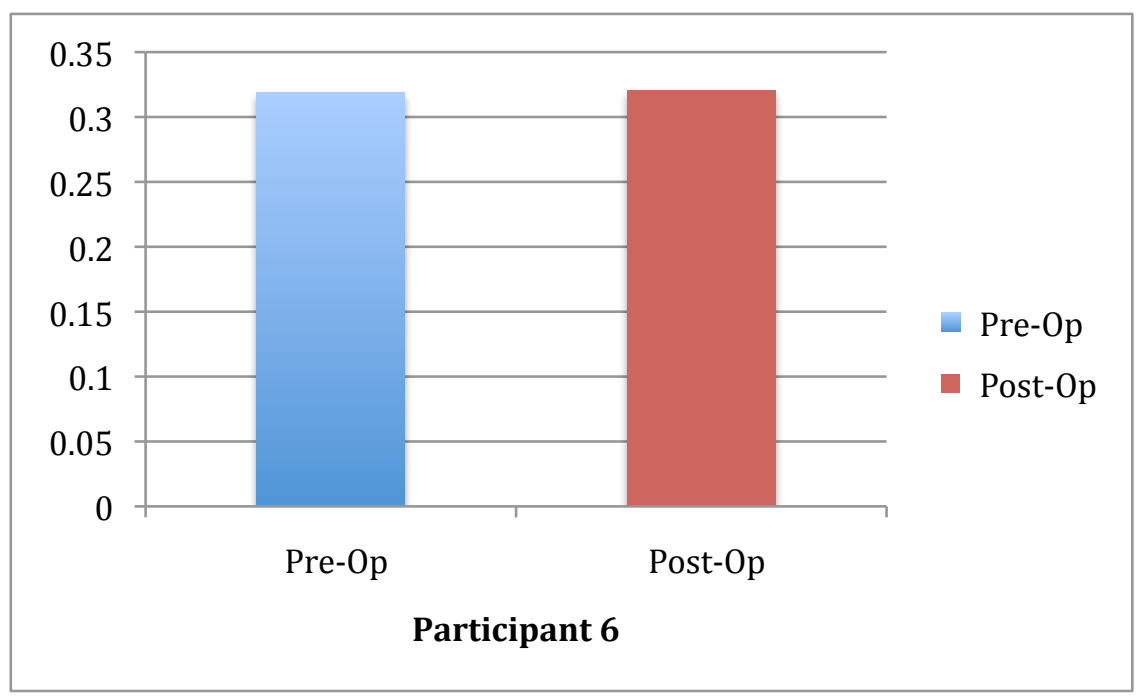

Figure A.35: Arch index comparison for stance of total peak area over time for two-foot stance

Pre-Op Arch Index: 0.32

Post-Op Arch Index: 0.32

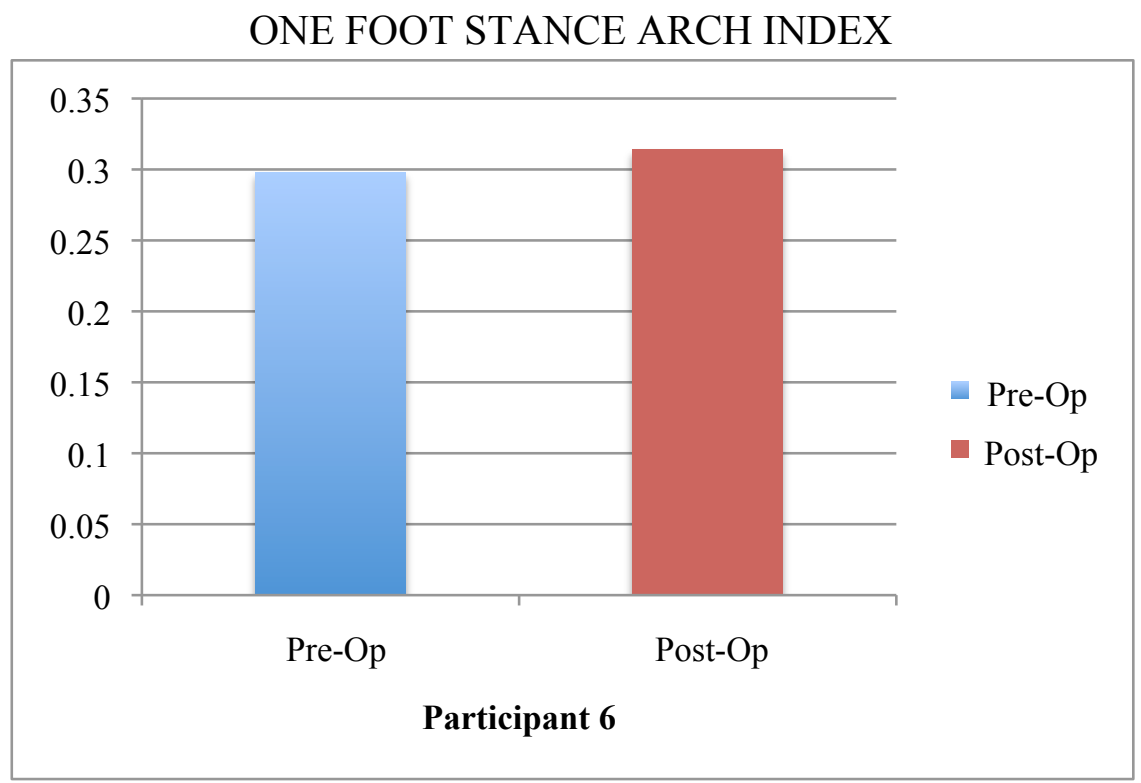

Figure A.36: Arch index comparison for stance of total peak area over time for afflicted foot stance.

Pre-Op Arch Index: 0.30

Post-Op Arch Index: 0.31 


\section{ARCH INDEX WALKING}

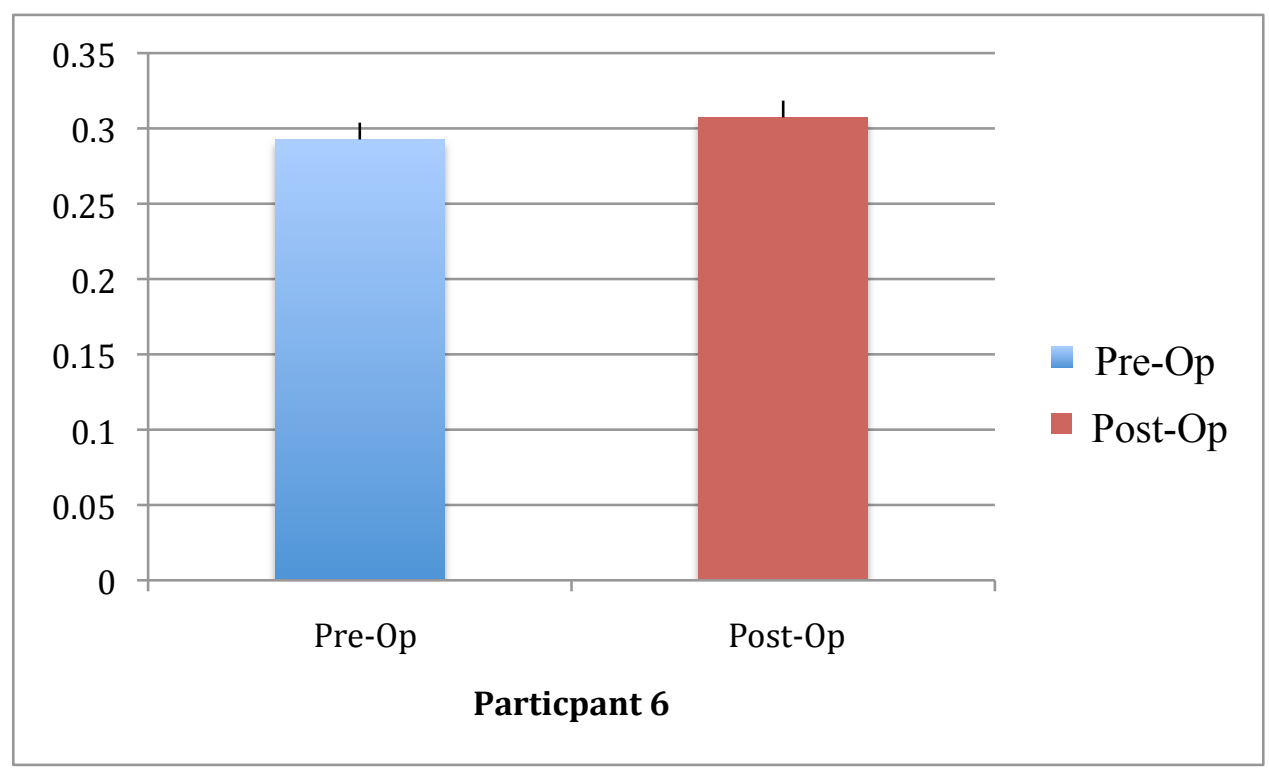

Figure A.37: Arch index measurements of the peak areas over time of three walking trials.

Pre-Op Arch index:

\begin{tabular}{|r|r|r|r|r|r|r|}
\hline Forefoot & Midfoot & Hindfoot & Total & $\begin{array}{l}\text { Arch } \\
\text { Index }\end{array}$ & $\begin{array}{l}\text { Average } \\
\text { AI }\end{array}$ & STD Dev \\
\hline 41 & 30 & 30 & 101 & 0.30 & & \\
\hline 41 & 30 & 29 & 100 & 0.30 & & \\
\hline 43 & 29 & 31 & 104 & 0.28 & 0.29 & 0.01 \\
\hline
\end{tabular}

Post-Op Arch index:

\begin{tabular}{|r|r|r|r|r|l|l|}
\hline Forefoot & Midfoot & Hindfoot & Total & $\begin{array}{r}\text { Arch } \\
\text { Index }\end{array}$ & $\begin{array}{l}\text { Average } \\
\text { AI }\end{array}$ & $\begin{array}{l}\text { STD } \\
\text { Dev }\end{array}$ \\
\hline 43 & 31 & 29 & 103 & 0.30 & & \\
\hline 43 & 35 & 31 & 110 & 0.32 & & \\
\hline 44 & 33 & 32 & 109 & 0.30 & 0.31 & 0.01 \\
\hline
\end{tabular}

Although arch index measures are supposed to decrease to normal levels, this participant did not decrease to "normal" levels. However, this participant was AfricanAmerican and may have a different "normal" shape to her foot that would make it more flat according to the arch index. Two-foot stance shows a slight increase in arch index 
and for one-foot stance more of an increase. Arch index may not be a good indicator for this participant as to the progress made for pre-op and post-op comparison since there was very little change in stance. However, it should be noted that this participant had an extremely difficult time standing pre-operatively on the afflicted foot and overcompensation occurred with the other standing leg and lateral shifting which will skew the measure for arch index.

Arch index measures for walking increased a significant amount from pre-op to post-op state. The arch index is supposed to decrease if the foot arch support is better. This may be due to the fact that the participant was compensation pre-operatively while walking and after surgery she felt more comfortable putting weight on her afflicted foot. 


\section{WORKS CITED}

Arangio, G., \& Salathe, E. (2009). A biomechanical analysis of posterior tibial tendon dysfunction, medical displacement calcaneal osteotomy and flexor digitorum longus transfer in adult acquired flat foot. Clinical Biomechanics , 385-90.

Blackman, A., Blevins, J., Sangeorzan, B., \& and Ledoux, W. (2009). Cadaveric flatfoot model: Ligament attenuation and Achilles tendon overpull. Journal of Orthopedic Research , 27 (12), 1547-54.

Blackman, A., Blevins, J., Sangeorzan, B., \& Ledoux, W. (2009). Cadaveric Flatfoot Model: Ligament attenuation and Achilles Tendon Overpull. Journal of Orthopedic Research , 1547-54.

Castro-Aragon, O. e. (2009). Ethnic Radiographic Foot Differences. Foot and Ankle International , 30, 57-61.

Cavanaugh, P., \& Morag, E. (1999). Structural and functional preictors of regional peak pressures under the foot during walking. Journal of Biomechanics , 359-70.

Cavanaugh, P., Morag, E., Boulton, A., Young, M., Deffner, K., \& Pammer, S. (1997). The relationship of static foot structure to dynamic foot function. Journal of Biomechanics , 243-50.

Chen, C.-H., Huang, M.-H., Chen, T.-W., Weng, M.-C., Lee, C.-L., \& Wang, G.-J. (2006). The Correlation Between Selected Measurements From Footprint and Radiograph of Flatfoot. Arch Physical Medcine Rehabilitation , 235-40.

Chi, T., Toolan, B., Sangeorzan, B., \& Hansen, S. (1999). The lateral column lengthening and medial column stabilization procedures. Clinical Orthopedics , 8190.

Coughlin, M. J., \& Kaz, A. (2009). Correlation of Harris Mats, Physical Exam, Pcitures and Radiographic Measurements in Adult Flatfoot Deformity. Foot and Ankle International , 30, 604-12.

Deland JT, P. A.-H. (2006). Posterior Tibial Tendon Insufficiency Results at Different Stages. HSS Journal , 2, 157-160.

Deland, J. T. (2008). Adult-acquired Flatfoot Deformity. Journal of the American Academy of Orthopedic Surgeons , 16 (7), 399-406. 
Deland, J. (2001). The Adult Acquired Flatfoot and Spring Ligament Complex: Pathology and Implications for Treatment. Foot and Ankle Clinics , 129-35.

Deland, J., de Asla, R., Sung, I., Ernberg, L., \& Potter, H. (2005). Posterior Tibial Tendon Insufficiency: Which Ligaments are Involved? Foot and Ankle International, 26 (6), 427-435.

Dunn J.E., L. C. (2004). Prevalence of Foot and Ankle Conditions in a Multiethnic Community Sample of Older Adults. American Journal of Epidemiology , 159, 491498.

Durrant, B., Chokalingham, N., \& Hashmi, F. (2011). Posterior Tibial Tendon Dysfunction: A Review. Journal of the American Podiatric Medial Association, 176-86. Elftman, H. (1960). The Transverse Tarsal Joint and its Control. Clinical Orthopaedic, $16,41-46$.

Ellis, S., Williams, B., Pavolov, H., \& Deland, J. (2011). Results of anatomic ligament reconstruction with tendon allograft. HSS Journal , 134-40.

Ellis, S., Yu, J., Johnson, H., Elliott, A., O'Malley, M., \& Deland, J. (2010). Plantar Pressures in Patient with and without Lateral Foot Pain After Lateral Column Lengthening. Journal of Bone and Joint Surgery , 81-91.

Faria, A., Gabriel, R., Abrantes, J., Bras, R., \& Moreira, H. (2010). The relationship of body mass index, age and triceps-surae musculotendinous stiffness witht eh foot arch structure of postmenopausal women. Clinical Biomechanics , 588-93.

Fishco, W. D., \& Cornwall, M. W. (2004). Gait Analysis After Talonavicular Joint Fusion: 2 Case Reports. The Journal of Foot and Ankle Surgery , 241-247.

Fung, Y. (1993). Biomechanics: Mechanical Properties of Living Tissues. New York, NY: Springer.

Gluck, G., Heckman, D., \& Selene, P. (2010). Tendon Disorders of the Foot and Ankle Part 3: Posterior Tibial Tendon. The American Journal of Sports Medicine , 2133-44.

Gray, H. (1918). Anatomy of the Human Body (20th edition ed.). (2. Bartleby.com, Ed.) Philadelphia: Lea \& Febiger.

Greisberg, J., Assal, M., Hansen, S., \& Sangeorzan, B. (2005). Isolated Medial Column Stabilization Improves Alignment in Adult-acquired Flatfoot. Clinical Orthopedica and Related Research , 197-202.

Guyton, e. a. (2001). Theorhetical limitations of the AOFAS scoring systems: An analysis using Monte Carlo Modeling. Foot and Ankle International . 
Hadfield, M. H., Snyder, J. W., Liacouras, P. C., Owen, J. R., Wayne, J. S., \& Adelaar, R. S. (2003). Effects of Medializing Calcaneal Osteotomy on Achilles Tendon Lengthening and Plantar Foot Pressures. Foot and Ankle International , 523-9.

Hadfield, M., Snyder, J., Liacouras, P., Owen, J., Wayne, J., \& Adelaar, R. (2005). The Effects of a Medializing Calcaneal Osteotomy With and Without Superior Translation On Achilles Tendon Elongation and Plantar Foot Pressures. Foot and Ankle International , 365-70.

Haight, H., Dahm, D., Smith, J., \& Krause, D. (2005). Measuring Standing Hindfoot Alignment; Reliability of Goniometric and Visual Measurements. Arch Phys Med Rehabilitation, 571-5.

Hicks, J. H. (1953). The Mechanics of the Foot: Part I. Journal of Anatomy , 345-357. Hill, K., Saar, W., Lee, T., \& Berlet, G. (2003). Stage II flatfoot: what fails and why. Foot Ankle Clinical , 8 (1), 91-104.

Hintermann, B. e. (1994). Foot movement and tendon excursion: an in vitro study. Foot and Ankle International , 15, 386-395.

Holmes GB Jr, M. R. (1992). Possible etilogical factors associated with rupture of the posterior tibial tendon (Vol. 13). Foot and Ankle.

Ibrahim, T., Beiri, A., Azzabi, M., Best, A., Taylor, G., \& Menon, D. (2007). Reliability and Validity of Subjective Component of AOFAS Clinical Rating Scales. Journal of Foot and Ankle Surgery , 65-74.

Imhauser, C., Abidi, N., Frankel, D., Gavin, K., \& Siegler, S. (2002). Biomechanical Evaluation of the Efficacy of External Stabilizers in the Conservative Treatment of Acquired Flatfoot Deformity. Foot and Ankle International , 727-37.

Imhauser, C., Siegler, S., Abidi, N., \& Frankel, D. (2004). The effect of posterior tibialis tendon dysfunction of the plantar pressure characterists and the kinematics of the arch and hindfoot. Clinical Biomechanics , 161-9.

Inman, V. (1976). The Joints of the Ankle. Baltimore, MD: Williams and Wilkins.

Jennings, M., \& Christensen, J. (2008). The Effects of Sectioning the Spring Ligament on Rearfoot Stability and Posterior Tibial Tendon Insufficiency. The Journal of Foot and Ankle Surgery , 47 (3), 219-24.

Johnson K.A, S. D. (1989). Tibialis Posterior Tendon Dysfunction. Clinical Orthopedic Relat Res, 239, 196-206. 
Joseph Jr., N. (2008). Film Critique of the Lower Extremity: Part 3. CCE Essentials.

Karasick, D., \& Schweitzer, M. (1993). Tear of The Posterior Tibial Tendon Causing Asymmetric Flatfoot: Radiologic Fidnigns. AJR, 1237-40.

Ker, R., Dimery, N., \& R, A. M. (1987). The spring in the arch of the human foot. Nature , 147-149.

Kitaoka, H., Ahn, T., Luo, Z., \& An, K. (1997). Stability of the arch of the foot. Foot and Ankle International , 18 (10), 644-8.

Klenerman, L., \& Wood, B. (2006). The Human Foot: A Companion to Clinical Studies. Washinton, DC: Springer-Verlag.

Ledoux, W., \& Howard, H. (2002). The distributed plantar vertical force of neutrally aligned and pes planus feet. Gait and Posture , 1-9.

Lee, D. (2009). Effects of Posterior Tibial Tendon Augmented With Biografts and Calcaneal Osteotomy in Stage II Adult-Acquired Flatfoot Deformity. Foot and Ankle Specialist , 2, 27-31.

Lee, K. M. (2010). Reliability and Validit of Radiographic Measurement in Hindfoot Varus and Valgus. The Journal of Bone and Joint Surgery , 2319-27.

Madeley, N., Wing, K., Topliss, C., Penner, M., Glazebrook, M., \& Younger, A. (2012). Responsiveness and validity of the SF-36, Anke Osteoarthritis scale, AOFAS Ankle Hindfoot Score, and Foot Function Index in end stage ankle arthritis. Foot and Ankle International , 57-63.

Masaheri, e. а. (2010 йил June). Reliability and Validity of Persian Version of FAAM to measure functional limitationsin patients with foot and ankle disorders.

Osteoarthritis and Cartilage .

Matheis, E.A., Spratley, E.M., Hayes, C.W., Adelaar, R.S., Wayne, J.S. Pre-Operative and Post-Operative Plantar Pressures in Stage IIb Posterior Tibial Tendon Insufficiency. ORS Seminar 2013. [Abstract ID: 1465638].

McCormack, A., Ching, R., \& Sangeorzan, B. (2001). Biomechanics of ProceduresUsed in Adult Flatfoot Deformity. Foot and Ankle Clinics , 15-23.

McMinn, R., Hutchings, R., \& Logan, B. (1996). Foot \& Ankle Anatomy. London: Mosby-Wolfe.

McPoll, T. C., Dupuis, L., \& M, C. (1999). Variability of plantar pressure data. A comparison of the two-step and midgait method. Journal of American Podiatry Medical Asscociation , 495-501. 
Menz, H., \& Morris, M. (2006). Clinical determinants of plantar forces and pressures during walking in older people. Gait and Posture , 229-36.

Menz, H., Fotoohabadi, M., Munteanu, S., Zammit, G., \& Gilheany, M. (2012). Plantar pressures and relative lesser metatarsal lengths in older people with and without forefoot pain. Journal of Orthopedic Research , 22-29.

Menz, H., Munteanu, S., Zammit, G., \& Landorf, K. (2010). Foot structure and function in older people with radiographic osteoarthritis of the medial midfoot. Osteoarthritis Cartilage , 317-322.

Murley, G., Menz, H., \& Landorf, K. (2009). A Protocol for Classifying Normal and Flat-arched Foot Posture for Research Studies Using Clinical and Radiographic Measurements. Journal of Foot and Ankle Research , 2 (22), 1-13.

Negahban, e. a. (2010). Reliability and Validity of Foot and Ankle Outcome Score: A Validation Study from Iran. Clinical Rheumatology .

Owen, P., \& Burke, J. (2011). The reliability of the associate plantinum digital foot scanner in measuring previously developed footprint characteristics: A Technical Note. Journal of Manipulative Physiol Therapy, 114-118.

Palsson, B., \& Bhatia, S. (2004). Tissue Engineering. Upper Saddle RIver, NJ: Pearson: Prentice Hall.

Prior, T. D. (1999). Biomechanical foot function: a podiatric perspective part I. Journal of Bodywork and Movement Therapies , 3, 74-84.

Richardson, D., Willers, J., Cohen, B., Davis, H., Jones, C., \& Anderson, R. (2009). Evaluation of the Hallux Morbidity of Single-Incision Flexor Hallucis Longus Tendon Transfer. Foot and Ankle International , 627-30.

Richie, D. H. (2007). Biomechanics and Clinical Analysis of Adult Acquired Flatfoot. Clinics in Podiatric Medicine and Surgery, 24, 617-644.

Richter, e. a. (2006). A New Foot and Ankle Outcome Score: Questionnaire Based, Subjective, Visual Analogue Scale, Validated Computerized. Foot and Ankle Surgery.

Rule, J., Yao, L., \& Seeger, L. (1993). Spring Ligament of the Ankle: Normal MR Anatomy. American Journal of Radiology , 1241-1244.

Schriebman, K. L., \& Bruce, R. (2009). Ankle and Foot. In J. R. Haaga, V. S. Dogra, M. Fortsing, R. C. Gilkeson, H. K. Ha, \& M. Sundaram, CT and MRI of the Whole Body (pp. 2207-2316). Philadelphia, PA: Elsevier. 
Scott G, e. a. (2007). Age-related difference in foot structure and function. Gait and Posture , 26, 68-75.

Scott, A., Hendry, T., Iaquinto, J., Owen, J., Wayne, J., \& Adelaar, R. (2007). Plantar pressure analysis in cadaver feet after bony procedures commonly used in the treatment of stage II posterior tibial tendon insufficiency. Foot and Ankle International , 1143-53.

Scranton, P. J., McMaster, J., \& Kelly, E. (1976). Dynamic fibular function: a new concept. Clinical Orthopedic Related Research , 76-81.

Shraeder, J. A., \& Lohmann Siegel, K. (2003). Nonoperative Management of Functional Hallux Limitus in a Patient with Rheumatoid Arthritis. Journal of the American Physical Therapy Association , 831-43.

Society, E. F. (2010). About the pathogensis of the so-called adult acquired pes planus : Editoral. Foot and Ankle Surgery, 1-22.

Tekscan, Incorporated. (2008). Tekscan Pressure Measurement System: HR Mat Research version 6.40. Boston, MA.

Toolan, B., Sangeorzan, B., \& Hansen, S. (1999). Complex Reconstruction for the Treatment of Dorsolateral Peritalar Subluxationof the Foot. Early Results after Distraction Arthrodesis with Calcaneocuboid Joint in Conjunction with Stabilization of and Transfer of the Flexor Digitorum Longus Tendon to the Midfoot to Treat Acquired Pes Planovalgus in Adults. Journal of Bone and Joint Surgery , 1545-60.

Urry, S., \& Wearing, S. (2001). A Comparison of Footprint Indexes Calculated from Ink and Electronic Footprints. Journal of American Podiatric Medical Association , 203-209.

Van Boerum, D., \& Sangeorzan, B. (2003). Biomechanics and pathophysiology of flat foot. Foot and Ankle Clinics , 8, 419-430.

Wheeless III, C. R., Nunley II, J. A., \& Urbaniak, J. R. (2011). Wheeless' Textbook of Orthopedics. Durham, NC, USA: Data Trace Internet Publishing LLC.

Yalcin, N., Esen, E., Kanatli, U., \& Yetkin, H. (2010). Evaluation of the medial longitudinal arch: a comparison between the dynamic plantar pressure measurement system and radiographic analysis. Acta Orthopadeica Traumatologia Turcica , 241-45.

Zammit, G., Menz, H., \& Shannon, M. (2010). Reliability of the TekScan MatScan system for the measurement of plantar forces and pressures during barefoot level walking in healthy adults. Journal of Foot and Ankle Research , 1-9. 
